\title{
An interdisciplinary primary care approach for frail older people : feasibility, effects and costs
}

Citation for published version (APA):

Metzelthin, S. F. (2014). An interdisciplinary primary care approach for frail older people : feasibility, effects and costs. [Doctoral Thesis, Maastricht University]. Maastricht University. https://doi.org/10.26481/dis.20140326sm

Document status and date:

Published: 01/01/2014

DOI:

10.26481/dis.20140326sm

Document Version:

Publisher's PDF, also known as Version of record

\section{Please check the document version of this publication:}

- A submitted manuscript is the version of the article upon submission and before peer-review. There can be important differences between the submitted version and the official published version of record. People interested in the research are advised to contact the author for the final version of the publication, or visit the DOI to the publisher's website.

- The final author version and the galley proof are versions of the publication after peer review.

- The final published version features the final layout of the paper including the volume, issue and page numbers.

Link to publication

\footnotetext{
General rights rights.

- You may freely distribute the URL identifying the publication in the public portal. please follow below link for the End User Agreement:

www.umlib.nl/taverne-license

Take down policy

If you believe that this document breaches copyright please contact us at:

repository@maastrichtuniversity.nl

providing details and we will investigate your claim.
}

Copyright and moral rights for the publications made accessible in the public portal are retained by the authors and/or other copyright owners and it is a condition of accessing publications that users recognise and abide by the legal requirements associated with these

- Users may download and print one copy of any publication from the public portal for the purpose of private study or research.

- You may not further distribute the material or use it for any profit-making activity or commercial gain

If the publication is distributed under the terms of Article $25 \mathrm{fa}$ of the Dutch Copyright Act, indicated by the "Taverne" license above, 


\section{An interdisciplinary primary care approach for frail older people}

FEASIBILITY, EFFECTS AND COSTS

The studies presented in this thesis were performed at the Department of Health Services Research, School for Public Health and Primary Care (CAPHRI), Maastricht University. CAPHRI is part of the Netherlands School of Primary Care Research (CaRe), which has been acknowledged since 1995 by the Royal Netherlands Academy of Arts and Sciences (KNAW)

The work described in this thesis was funded by The Dutch National Care for the Elderly Programme, coordinated and sponsored by The Netherlands Organisation for Health Research and Development (ZonMw). Printing of this thesis was financially supported by Centres of Research on Autonomy and Participation, and Technology in Health Care, Zuyd University of Applied Sciences, School for Public Health and Primary Care (CAPHRI),

Maastricht University, Medical Coordination Centre Omnes, Meditta and ABN AMRO.

\section{Design and lay-out}

Promotie In Zicht, Arnhem

Ipskamp Drukkers, Enschede

ISBN

978-94-6191-982-3

\section{ilke Friederike Metzelthin}

ter verkrijging van de graad van doctor aan de Universiteit Maastricht, op gezag van de Rector Magnificus, Prof dr. L.L.G. Soete volgens het besluit van het College van Decanen, in het openbaar te verdedigen

op woensdag 26 maart 2014 om 16.00 uur 
Prof. dr. G.I.J.M. Kempen

Prof. dr. L.P. de Witte

Chapter 1 General introduction

Copromotor

Dr. H.I.J. van Rossum

Beoordelingscommissie

Prof. dr. J.F.M. Metsemakers (voorzitter)

Prof. dr. C.D. Dirksen

Prof. dr. M.J. Schuurmans (University of Utrecht)

Prof. dr. A.E. Stuck (University of Bern, Switserland)

Prof. dr. T. van der Weijden
Chapter 2 The psychometric properties of three self-report screening instruments for identifying frail older people in the community Published in BMC Public Health. 2010;10:176

Chapter 3 Interventions to prevent disability in frail community-dwelling older people: an overview

Published in European Journal of Ageing. 2010;7:37-55

Chapter 4 The reduction of disability in community-dwelling frail older people: design of a two-arm cluster randomised controlled trial Published in BMC Public Health. 2010;10:51

Chapter 5 A nurse-led interdisciplinary primary care approach to prevent disability among community-dwelling frail older people: a large-scale process evaluation

Published in International Journal of Nursing Studies. 2013;50(9):1184-96

Chapter 6 Effectiveness of an interdisciplinary primary care approach to reduce disability in community-dwelling frail older people: a cluster randomised controlled trial

Published in British Medical Journal 2013;347:f5264

Chapter 7 Cost-effectiveness of an interdisciplinary primary care approach to reduce disability in community-dwelling frail older people: a cluster randomised controlled tria Under review

\section{Chapter 8 General Discussion}

Summary

Nederlandse samenvatting

Deutsche Zusammenfassung

Dankwoord | Dankwort

About the author

List of publications

Appendix A disability prevention programme for community-dwelling frail older people

Published in Clinical Rehabilitation. 2011:25:1-12 
General introduction

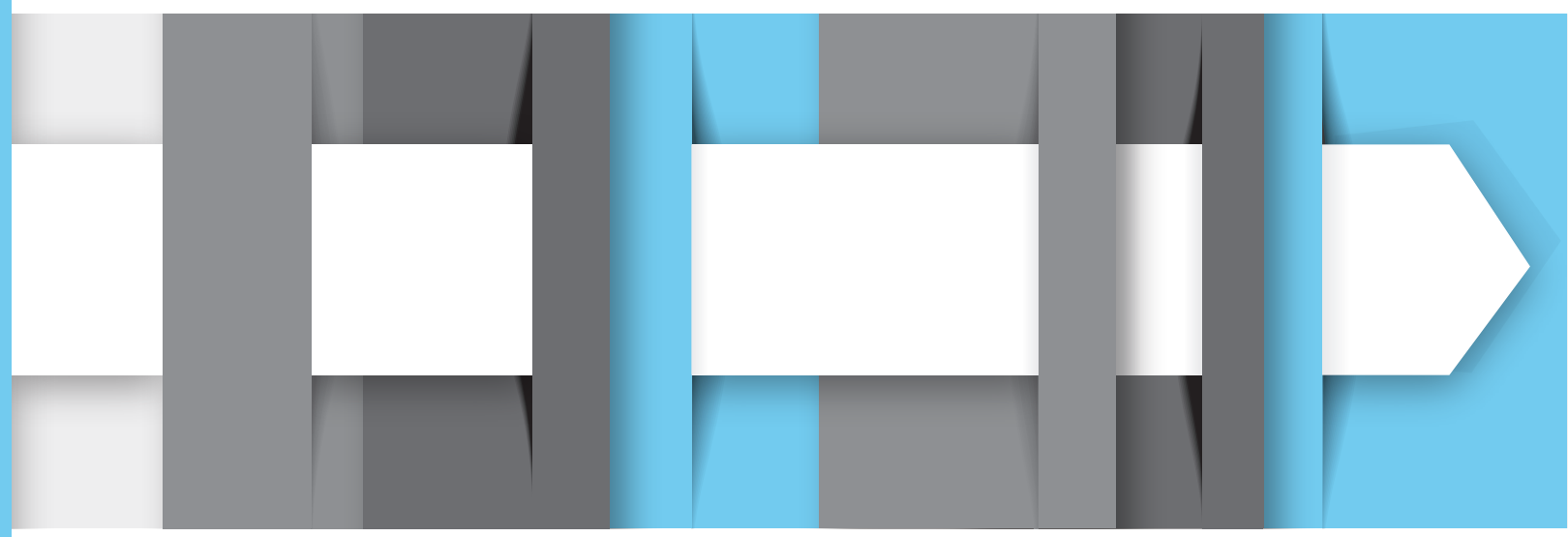




\section{Introduction}

In our ageing society, care for older people is one of the greatest challenges in healthcare. In the Netherlands, the proportion of older people ( $\geq 65$ years) will rise from 2.4 million (16\% of the total population) in 2010 to 4.6 million (25\% of the total population) in 2050. ${ }^{1}$ In 2008, the Dutch Ministry of Health, Welfare and Sport launched the National Care for the Elderly Program, an initiative to improve the quality of care for frail older people. As part of the program, this thesis focuses on the evaluation of a pro-active primary care approach to reduce disability and to prevent (further) functional decline in community-dwelling frail older people.

\section{Frailty}

Within an ageing population, the number of frail older people will increase. ${ }^{2}$ Frailty appears when the reserve capacity of older people has decreased to a critically low point, at which even small disturbances can lead to a series of complications. ${ }^{3}$ Frailty can be positioned on a continuum from pre-frail to frail. The level of frailty can change over time in either direction, meaning that individuals can become more or less frail.4 In the on-going discussion about the conceptualisation of frailty two main streams can be distinguished: firstly, the frailty phenotype, which focuses on the physical domain of functioning taking into account slow walking speed, impaired grip strength, declining activity levels, exhaustion and unintended weight loss;,3,5 secondly, a multidimensional perspective that incorporates physical, psychological and socia deficits. ${ }^{6,7}$ Within each dimension various (risk) factors or determinants for frailty exist. A well-known example is the Frailty Index, also acknowledged as the deficit accumulation model. ${ }^{8}$ It conceptualises frailty as a multidimensional risk state that can be measured by the quantity of deficits. The more deficits an older person has, the higher his or her likelihood of being frail. ${ }^{8}$ In the literature, the prevalence rates of frailty range considerably, as frailty is defined and measured in different ways and is assessed in different populations. ${ }^{9-12} \mathrm{~A}$ recent systematic review by Collard and colleague $\mathrm{s}^{10}$ identified prevalence rates in community-dwelling older people ranging from 4 to $59 \%$. On average, $11 \%$ of community-dwelling older people ( $\geq 65$ years) were frail and $42 \%$ were pre-frail.10 Despite the on-going discussion about the conceptualisation of frailty, there is consensus that frail older people have an increased risk of adverse health outcomes, such as acute and chronic diseases, falls, disability institutionalisation and death. ${ }^{10,13}$ The concept of frailty is important for clinicians to understand the heterogeneity and inequalities of ageing, as individuals with the same chronological age may vary widely in health, functional status and need for healthcare utilisation. 
Identification of frail older people

To reduce disability and prevent (further) functional decline frail older people have to be identified in time. Consequently, appropriate screening methods are of increasing importance. One possible screening strategy is regular screening of all individuals of a certain age, which is also known as population screening. ${ }^{13}$ For this purpose the age of 70 years seems to be reasonable, as rapid decline of physiological function occurs after this age.13 In contrast, based on an opportunistic screening approach, older people are screened during regular contact with a healthcare provider (e.g. GP consultation, admission to hospital).$^{13}$ Irrespective of the screening strategy, there are numerous performance tests, questionnaires and indices available to measure frailty. ${ }^{4}$ Some of them are based on a physiological definition of frailty, while others are developed from a multidimensional perspective. Instruments that focus on physical functioning provide lower and more consistent prevalence rates. However, a multidimensional approach provides information about deficits and care needs in different areas of functioning, which might be of clinical relevance. ${ }^{10}$ Frailty instruments can also be distinguished in self-report versus performance-based measures. Self-report measures are believed to be an efficient method for reaching large populations and for providing high response rates and reliable and valid answers. ${ }^{14}$ However, performance-based measures are less influenced by socio-demographic variables, personality and cognitive and affective factors. ${ }^{14-16}$ Finally, the choice of a particular instrument depends on the setting, the aim of the measurement, the qualities of the person who administers the instrument and the time available. ${ }^{4}$

Reduction of disability and prevention of (further) functional decline The identification of frail older people is only useful if effective interventions are available to reverse frailty or to reduce or prevent its adverse health outcomes. ${ }^{13}$ One major health outcome is disability, which is defined as difficulty or dependency in the execution of daily activities. ${ }^{5}$ Disability can more specifically be related to self-care activities and meaningful activities that are essential for independent living and one's quality of life. ${ }^{5}$ Functioning of older people may be influenced by their physiological or psychological state, however, interactions with the physical and social environment can also stimulate or hinder participation in daily activities. ${ }^{4}$ In other words, disability reflects the gap between an individual's abilities and the environment in which that person functions..$^{17}$ Disability can be considered as a dynamic process, which means that older people can recover to a less or non-disabled state. ${ }^{18}$ While disability itself is an adverse health outcome, it is also a risk factor for other adverse events, It increases the risk of diseases, further functional decline, injuries and falls, increased healthcare utilisation, including informal and formal care, and mortality. ${ }^{19}$ Consequently, disability in older people affects their quality of life and has a major impact on their social network and the entire healthcare system. ${ }^{19}$ Thus, effective interventions with regard to disability and other adverse health outcomes have the potential to substantially diminish the burden caused by frailty.

Community-based care in frail older people

Western countries with ageing populations, such as the Netherlands, have to dea with an increasing demand for care, while financial resources and manpower are shrinking. ${ }^{20}$ The proportion of older people in Dutch nursing homes and homes for the elderly is decreasing and community-based care is becoming more important. ${ }^{2}$ The majority of older people, even with fragile health and faced with challenging social situations, prefer to stay at home for as long as possible..$^{18}$ Active and independent participation in daily life is highly important to them..$^{22}$ In addition, evidence suggests that community-based care may achieve better outcomes at lower costs compared to institutionalisation. ${ }^{23}$ Consequently, The Dutch Council for Public Health and Care emphasises the importance of structural healthcare reform based on scientific evidence, to improve. ${ }^{24}$ Innovative initiatives are needed to provide cost-effective community-based care. ${ }^{25,26}$

During the past decades much research on complex interventions targeting community-dwelling (frail) older people has been conducted, with a substantial amount of studies in the field of preventive home visiting programmes. Since 2000 several meta-analyses, ${ }^{27-29}$ systematic reviews, ${ }^{30-32}$ and literature reviews ${ }^{18,33}$ have been published. The reviews evaluated a range of interventions (e.g. multidimensional geriatric assessment, care planning, organisation and monitoring, health promotion, self-management support, nursing services and referrals to other services) carried out by various professionals (e.g. general practitioners, nurses, allied professionals). The aim of these interventions is to proactively detect modifiable risk factors and worsening health conditions to reduce or prevent disability and other negative health outcomes, health care utilisation and related costs. However, the reviews produced inconsistent and conflicting results regarding their effectiveness and little is known about their cost-effectiveness. It is not yet clear whether such interventions have the potential to reduce disability or prevent (further) functional decline in frail older people or which components are most promising

Recent developments in the Dutch healthcare system

As stated in the beginning the National Care for the Elderly Program ${ }^{34}$ aims to improve the quality of care for older people by developing coherent care that is better suited to their individual needs. First, the eight University Medical Centres in the Netherlands were asked to create networks in their region, consisting of relevant stakeholders in healthcare and welfare services and patient representatives, to increase the infrastructure for healthcare delivery. Second, each regional network has conducted a number of research projects to improve the quality and coherence of care for frail 
older people. ${ }^{34}$ In the south of the Netherlands the ACZIO (Academisch Centrum ZorgInnovatie Ouderenzorg) network was created. This thesis reports on one of their research projects evaluating the 'Prevention of Care' (PoC) approach. The PoC approach is an interdisciplinary primary care approach for community-dwelling frail older people. The development of the programme was based on the Intervention Mapping protocol ${ }^{35}$ for developing health promotion programmes. In the beginning two literature reviews were conducted to provide an extensive overview of existing interventions for community-dwelling frail older people, which report on disability as outcome measure. Various interventions were identified with most of them in the field of comprehensive geriatric assessment and physical exercise programmes. ${ }^{36,37}$ Based on these reviews we suggested that community-based interventions for fral older people should be conducted by an interdisciplinary primary care team, involving (population) screening, individualised assessments and interventions (tailor-made care), self-management support, engagement in meaningful activities, case management and long-term follow-up. These elements were combined in the PoC approach. A multidisciplinary task group, consisting of researchers and healthcare professionals (general practitioner, practice nurse, geriatrician, nursing home physician, geriatric clinical nurse specialist, physiotherapist and occupationa therapist, technology expert) developed the protocol and materials of the PoC approach. Finally, a pilot study $(n=41)$ was conducted providing promising results with regard to its feasibility. ${ }^{38}$ Older people appreciated the attention they got and felt supported in reaching their goals and in handling future disability. Healthcare professionals reported that the approach provided a useful structure for geriatric primary care; interdisciplinary collaboration, a focus on meaningful activities and selfmanagement support were stimulated by the approach. ${ }^{38}$ However, the effectiveness of the PoC approach with regard to disability and various related outcomes was not studied.

\section{Aims and outline}

The main objective of this thesis is to evaluate the surplus value of the PoC approach. The underlying aims of this thesis are:

- To evaluate and compare the psychometric properties of three screening instruments to identify frail older people.

- To evaluate the feasibility of the PoC approach

To evaluate the PoC approach with regard to disability (primary outcome) and other secondary outcomes, including healthcare utilisation and costs.

Findings may lead to more insight into effective methods to identify communitydwelling frail older people. In addition, findings will contribute to the knowledge about community-based interventions to reduce disability and prevent (further) functional decline and other adverse health outcomes.

Chapter 2 reports on the internal consistency and construct validity of three frailty instruments used for postal screening to identify community-dwelling frail older people: the Groningen Frailty Indicator, ${ }^{39}$ the Tilburg Frailty Indicator ${ }^{40}$ and the Sherbrook Postal Questionnaire. ${ }^{41}$ Chapter 3 gives an overview of interventions that have been studied in randomised controlled and clinical controlled trials aimed at community-dwelling frail older people with disability as an outcome measure. The review was conducted to identify and summarise promising components for future intervention programmes. The design of the cluster randomised trial is described in Chapter 4 . Information is provided on the recruitment of participants, the randomisation procedure, the intervention under study, data collection and data analyses. Chapte 5 is about the results of the process evaluation, which was conducted alongside the trial. Data was collected among older people and healthcare professionals to examine the extent of implementing the intervention protocol and to gain insight into healthcare professionals' and frail older people's experiences regarding benefits, burdens, stimulating factors and barriers. In Chapter 6 the effects of the PoC approach with regard to disability (primary outcome) and depressive symptomatology, socia support interactions, fear of falling and social participation are presented. Data were collected after 6, 12 and 24 months among 346 community-dwelling frail older people. The outcomes of the cost-effectiveness (CEA) and cost-utility analysis (CUA) are reported in Chapter 7 . The evaluation was performed from a societal perspective with a time horizon of 24 months. Finally, Chapter 8 provides a summary and discussion of the main findings, followed by implications for practice and future research. The PoC approach is described in more detail in the Appendix. 


\section{References}

1. CBS. Bevolkingsprognose 2010-2060: sterkere vergrizzing, langere levensduur. Den Haag: Centraa Bureau voor Statistiek;2010.

2. Lally F, Crome P. Understanding frailty. Postgrad Med J. 2007;83(975):16-20.

3. Fried LP, Tangen CM, Walston J, Newman AB, Hirsch C, Gottdiener J, Seeman T, Tracy R, Kop WJ, Burke G. McBurnie MA. Frailty in older adults: evidence for a phenotype J Gerontol A Biol Sci Med Sci. 2001;56(3):M146-56.

4. De Vries NM, Staal JB, van Ravensberg CD, Hobbelen JS, Olde Rikkert MG, Nijhuis-van der Sanden MW. Outcome instruments to measure frailty: a systematic review. Ageing Res Rev. 2011;10(1):104-14.

5. Fried LP, Ferrucci L, Darer J, Williamson JD, Anderson G. Untangling the concepts of disability, frailty, and comorbidity: implications for improved targeting and care. J Gerontol A Biol Sci Med Sci 2004:59(3):255-63.

6. Bergman H, Beland F, Karunanthan S, Hummel S, Hogan D, Wolfson C. Developing a working framework for understanding frailty. (English translation of article published in) Gerontol Soc. 2004;109:15-29.

7. Gobbens RJ, van Assen MA, Luijkx KG, Schols JM. Testing an integral conceptual model of frailty. J Adv Nurs. 2012;68(9):2047-60

8. Rockwood K, Mitnitski A. Frailty in relation to the accumulation of deficits. $J$ Gerontol A Biol Sci Med Sci 2007:62(7):722-7.

9. Bergman H, Ferrucci L, Guralnik J, Hogan DB, Hummel S, Karunananthan S, Wolfson C. Frailty: an emerging research and clinical paradigm - issues and controversies. J Gerontol A Biol Sci Med Sci. 2007:62(7):731-7.

10. Collard RM, Boter H, Schoevers RA, Oude Voshaar RC. Prevalence of frailty in community-dwelling older persons: a systematic review. J Am Geriatr Soc. 2012;60(8):1487-92

11. Lacas A, Rockwood K. Frailty in primary care: a review of its conceptualization and implications for practice. BMC Med 2012;10(4):1-9.

作 2003;44(1):58-68

3. Pijpers E, Ferreira I, Stehouwer CDA, Nieuwenhuijzen Kruseman AC. The frailty dilemma. Review of the predictive accuracy of major frailty scores. Eur J Intern Med. 2012;23(2):118-23.

14. Martin FC, Brighton P. Frailty: different tools for different purposes? Age Ageing. 2008;37(2):129-31

gS SR, Curb JD. Physical performance measures in aging research. Gerontol. 1989;44(5):M141-6.

16. Kempen GIJM, van Heuvelen MJG, van den Brink RHS, Kooijman AC, Klein M, Houx PJ, Ormel J. Factors affecting contrasting results between self-reported and performance-based levels of physical limitation Age Ageing. 1996;25(6):458-64

17. Verbrugge LM, Jetle AM. The disablement process. Soc SciMed. 1994,38(1):1-14

18. Liebel DV, Friedman B, Watson NM, Powers BA. Review of nurse home visting interventions for community-dwelling older persons with existing disability. Med Care Res Rev. 2009;66(2):119-46.

19. Guralnik JM, Fried LP, Salive ME. Disability as a public health outcome in the aging population. Annu Rer Public Health. 1996;17:25-46.

20. Tappenden P, Campbell F, Rawdin A, Wong R, Kalita N. The clinical effectiveness and cost-effectiveness of home-based nurse-led health promotion for older people: a systematic review. Health Technol Assess. 2012;16(20):1-72.

21. Klerk MD. Zorg in de laatste jaren. Gezondheid en hulpgebruik in verzorgings- en verpleeghuizen 2000-2008. The Hague, The Netherlands: Sociaal en Cultureel Planbureau;2011.

22. Phelan EA, Anderson L.A., LaCroix AZ, Larson EB. Older adults' views of 'succesfull ageing' - how do they compare with researchers' definitions? J Am Geriatr Soc. 2004;52(2):211-6.

23. Woolhandler S, Campbell T, Himmelstein DU. Costs of health care administration in the United States and Canada N Engl J Med. 2003;349:768-75

24. RVZ. Redzaam ouder. Zorg voor niet-redzame ouderen vraagt om voorzorg door iedereen. The Hague, The Netherlands: Raad voor de Volksgezondheid \& Zorg:2012.
25. Keating $\mathrm{N}$, Ottinowski P, Wenger C, Fast J, Derksen L. Understanding the caring capacity of informal networks of frail seniors: a case for care networks. Ageing Soc. 2003;23(1):115-27.

26. Markle-Reid M. Browne G, Weir R, Gafni A, Roberts J, Henderson SR. The effectiveness and efficiency of home-based nursing health promotion for older people; review of the literature. Med Care Res Rev. 2006;63(5):531-69

27. Elkan R, Kendrick D. Dewey M. Hewitt M. Robinson J. Blair M. Williams D. K. B. Effectiveness of home based support for older people. systematic review and meta-analysis. BMJ. 2001:323(7315):719-25.

28. Stuck $A E$ E Egger M. Hammer A. Minder CE, Beck JC. Home visits to prevent nursing home admisson and functional decline in eldely people: systematic review and meta-regression analysis. JAMA. 2002;287(8):1022-8.

29. Beswick AD, Rees K Dieppe P. Ayis S, Gooberman-Hill R. Horwood J Ebrahim S Complex interventions independent living in elderly people: a systematic review and meta-ana sis Lancet 2008 $371(0014) 72535$.

. Bouman A, van Rossum E, Nelemans P., Kempen GIJM, Knipschild P. Effects of intensive home visiting status: a systematic review. BMC Health Serv Res. 2008;8:74.

31. Huss A, Stuck AE Rubenstein LZ, Egger M, Clough-Gorr KM. Multidimensional preventive home visi programs for community-dwelling older adults: a systematic review and meta analysis of randomized control

32. Van Haastregt JC, Diederiks JP, van Rossum E, de Witte LP, Crebolder HF. Effects of preventive home visits to elderly people living in the community. systematic review. BMJ. 2000;320(7237):754-8.

33. Hallberg IR, Kristensson J. Preventive home care of frail older people: a review of recent case management studies. J Clin Nurs. 2004;13(6B):112-20.

34. Nationaal Programma Ouderenzorg 2008-2011. Retrieved from http://www.nationaalprogrammaouderenzorg.nl//english/the-national-care-for-the-elderly-programme/. Accessed May 2013.

35. Bartholomew L K Parcel GS, Kok G. Gottlieb NH. Planning health promotion programs: an intervention mapping approach. Vol 2nd edition: Jossey-Bass; 2006.

36. Daniels R, Metzelthin $\mathrm{S}$, Van Rossum E, De Witte L, Van den Heuvel W. Interventions to prevent disability in frail community-dwelling older persons: an overview Eur J Ageing 2010;7(1):37-55

37. Daniels $\mathrm{R}$, van Rossum E, de Witte L, Kempen Gl, van den Heuvel W. Interventions to prevent disability in frail community-dwelling elderly: a systematic review. BMC Health Serv Res. 2008;8(1):278

38. Daniels R. Frail Elderly - Identification and disability prevention in primary care [PhD Thesis]. Maastricht School for Public Health and Primary Care, Maastricht University; 201

39. Steverink N, Slaets JPJ, Schuurmans H, van Lis M. Measuring frailty: development and testing of the Groningen Frailty Indicator (GFI). Gerontologist. 2001;:41(1):236-7.

40. Gobbens RJJ, van Assen MALM, Luijkx KG, Wijnen-Sponselee MT, Schols JMGA. The Tilburg Frailty Indicator: psychometric properties. J Am Med Dir Assoc. 2010;11(5):344-55.

41. Hebert R, Bravo G, Korner-Bitensky N, Voyer L. Predictive validity of a postal questionnaire for screening community-dwelling elderly individuals at risk of functional decline. Age Ageing. 1996;25(2):159-67. 


\section{2}

The psychometric properties

of three self-report screening instruments

for identifying frail older people

in the community

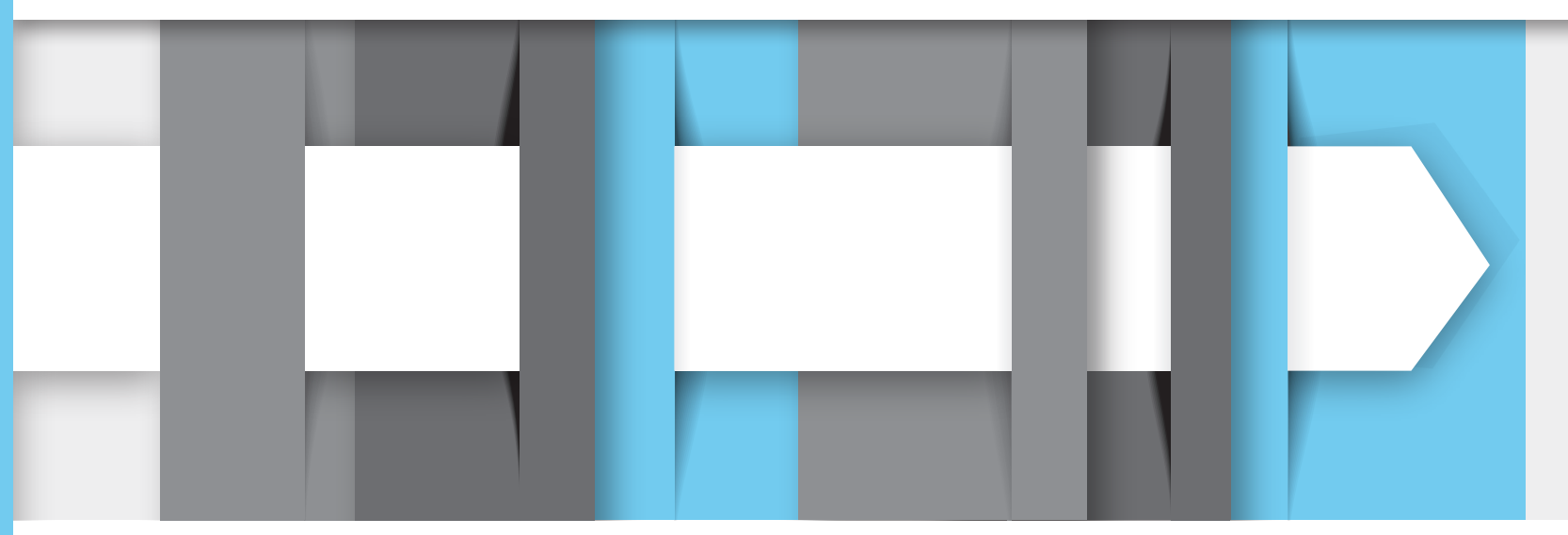

This chapter was published as:

Metzelthin, SF, Daniëls, R, van Rossum, E, de Witte, LP, van den Heuvel, W, Kempen, GIJM BMC Public Health 2010;10:51 


\section{Abstract}

Background: Frailty is highly prevalent in older people. Its serious adverse consequences, such as disability, are considered to be a public health problem. Therefore, disability prevention in community-dwelling frail older people is considered to be a priority for research and clinical practice in geriatric care. With regard to disability prevention, valid screening instruments are needed to identify frail older people in time. The aim of this study was to evaluate and compare the psychometric properties of three screening instruments: the Groningen Frailty Indicator (GFI), the Tilburg Frailty Indicator (TFI) and the Sherbrooke Postal Questionnaire (SPQ). For validation purposes the Groningen Activity Restriction Scale (GARS) was added. Methods: A questionnaire was sent to 687 community-dwelling older people ( $\geq 70$ years). Agreement between instruments, internal consistency, and construct validity of instruments were evaluated and compared.

Results: The response rate was $77 \%$. Prevalence estimates of frailty ranged from $40 \%$ to $59 \%$. The highest agreement was found between the GFI and the TFI (Cohen's kappa $=0.74)$. Cronbach's alpha for the GFI, the TFI and the SPQ was $0.73,0.79$ and 0.26 , respectively. Scores on the three instruments correlated significantly with each other (GFI-TFI, $r=0.87$; GFI-SPQ, $r=0.47$; TFI-SPQ, $r=0.42$ ) and with the GARS (GFI-GARS, $r=0.57$; TFI-GARS, $r=0.61$; SPQ-GARS, $r=0.46$ ). The GFI and the TF scores were, as expected, significantly related to age, sex, education and income. Conclusions: The GFI and the TFI showed high internal consistency and construc validity in contrast to the SPQ. Based on these findings it is not yet possible to conclude whether the GFI or the TFI should be preferred; data on the predictive values of both instruments are needed. The SPQ seems less appropriate for postal screening of frailty among community-dwelling older people.

\section{Background}

Frailty is highly prevalent in older people. Up to $40 \%$ of older people can be considered as frail and an increasing trend can be expected. ${ }^{1}$ Next to its high prevalence, frailty is characterized by its seriousness as it is related to increased risk of adverse health outcomes such as disability. ${ }^{2-4}$ Disability is defined as difficulty or dependency in the execution of activities of daily living and it is associated with increased health service utilisation and related costs. Frailty and disability are separate but overlapping concepts. On the one hand, frailty predicts disability. On the other hand, disability may well exacerbate frailty. ${ }^{5}$ With regard to a growing frail population and limited health care expenditures, disability in community-dwelling frail older people is suggested to be a public health problem. ${ }^{3}$ Therefore disability prevention in communitydwelling frail older people is considered to be a priority for research and clinical practice in geriatric care. ${ }^{6}$

Several authors emphasize a two-step approach in preventive interventions for community-dwelling frail older people, in which screening is followed by extensive assessment. With valid (screening) instruments to identify frail older people this approach may avoid costs and the unnecessary assessment of healthy people. ${ }^{78}$ During the last few decades, various instruments, based on various definitions, have been developed to detect frailty. This has led to a diversity of prevalence estimates of frailty. ${ }^{4}$ Little is yet known about the reliability and validity of these instruments and no gold standard exists. Therefore, more insight into the psychometric properties of frailty instruments is relevant for geriatric care and research in this area.

Frailty instruments have been developed from the point of view of different perspectives on frailty. ${ }^{9}$ From a physiological perspective physical frailty markers such as unintentional weight loss or weakness (grip strength), are used to identify frail older people. ${ }^{5}$ Next to physical factors, a multifactorial perspective on frailty also takes psychological, social and environmental factors into account..$^{10}$ An example of such an instrument is the Frailty Index, ${ }^{11,12}$ which combines, for example, physical frailty markers such as weight loss and grip strength with other factors such as cognition, mood or limitations in (instrumental) activities of daily living. Frailty may be elaborated more sharply if it is described from a physiological perspective. However the usefulness of this perspective in daily practice is questioned, as frailty cannot be separated from other factors such as cognition, mood or social support. ${ }^{9}$

Frailty instruments can be divided into self-report and performance-based instruments. ${ }^{9}$ It is assumed that performance-based instruments provide more precise and valid answers.13,14 Although they are less influenced by socio-demographic variables, personality and cognitive and affective factors, they are more sensitive to non- response, changes in time and differences in the execution of activities. Furthermore, they are less easy to conduct and time-consuming..$^{13-15}$ Self-report 
measures are believed to be an efficient method for reaching large groups and for providing high response rates and reliable and valid answers. ${ }^{15}$

In this study, we present the psychometric properties of frailty instruments that define frailty from a multifactorial perspective and are applicable for postal screening of community-dwelling older people. Given this objective and the target population, the Groningen Frailty Indicator (GFI) ${ }^{16}$, the Tilburg Frailty Indicator $(\mathrm{TFI})^{17}$, and the Sherbrooke Postal Questionnaire (SPQ) ${ }^{8}$ were chosen. The GFI and the SPQ have been used in previous studies for the purpose of postal screening. ${ }^{18-22}$ However, empirical evidence about the psychometric properties of the GFI, the TFI, and the $\mathrm{SPQ}$ is still scarce. The purpose of the present study was to evaluate and compare their psychometric properties.

\section{Methods}

Study design and participants

A cross-sectional study was conducted in a sample of 687 community-dwelling olde people living in the areas of Limburg and Utrecht in the Netherlands. Older people were identified between November 2008 and April 2009 from the panels of three general practitioners (GPs). All persons aged 70 years or above from each of the panels were invited by these GPs to participate in the study and to fill in a short questionnaire. The questionnaire included the three frailty instruments (GFI, TFI $\mathrm{SPQ}$ ) and an instrument that measures disability with respect to (instrumental) activities of daily living: the Groningen Activity Restriction Scale (GARS). ${ }^{23}$ After two weeks a reminder was sent to non-respondents. Participants of the study were well informed about the study in a patient information letter that accompanied the questionnaire. The information letter was formulated according to the guidelines of good clinical practice. Participants had to give written informed consent. The study did not require ethical approval. The postal procedure, including the questionnaire, has proven to be feasible for postal screening in a pilot study. ${ }^{24}$

Data collection

The three frailty instruments and the disability measure are briefly described below.

Measures

The Groningen Frailty Indicator (GFI), developed by Steverink and colleagues, ${ }^{16}$ is a screening instrument for determining the level of frailty. It consists of fifteen items and focuses on the loss of functions and resources in four domains of functioning physical (nine items), cognitive (one item), social (three items) and psychological (two items). Most items can be answered with 'yes' or 'no'. For the cognitive and psychosocial items the option 'sometimes' is added. Scores on the GFI range from zero to fifteen. A total score of four or higher is considered as moderately to severely frail. ${ }^{16,21} \mathrm{~A}$ study by Steverink and colleagues ${ }^{16}$ suggested that the GFI is an internally consistent scale with positive indications for construct and clinical validity.

The Tilburg Frailty Indicator (TFI) has recently been described by Gobbens and colleagues $^{17}$ and consists of two subscales. The first subscale (ten items) comprises determinants of frailty, for example, socio-demographic data and data abou life-events and chronic diseases. Socio-demographic data (age, sex, educationa level and income) were used for validation purposes. The analyses of psychometric properties focus on the second subscale, which determines the level of frailty. This subscale consists of fifteen items that are about physical (eight items), social (three items) and psychological factors (four items), including one item which is about cognition. Most items can be answered with 'yes' or 'no'. For the psychological items the option 'sometimes' is added. Scores for the TFI range from zero to fifteen. A score of five or higher is considered to be associated with frailty. ${ }^{17}$

The Sherbrooke Postal Questionnaire (SPQ) was developed by Hébert and colleagues ${ }^{8}$ and consists of six items aiming to identify frail older people in the community. The items focus on the physical (four items), social (one item) and cognitive (one item) domains of functioning. Items can be answered with 'yes' or 'no' Scores range from zero to six. Those older persons scoring two or higher, or who do not respond to the questionnaire, are considered to have an increased risk for functional decline and therefore are assumed to be frail. It should be noted that in the present study non-respondents were excluded from the analyses. In a Canadian sample of community-dwelling older people, predictive validity with regard to functional decline has been found. ${ }^{8}$ There are also indications for its predictive validity with regard to requirements for further assessment, ${ }^{20}$ use of emergency services ${ }^{22}$ and mortality. ${ }^{19}$

The Groningen Activity and Restriction Scale (GARS) ${ }^{23}$ is a valid and reliable instrument and consists of two subscales. The first subscale is about activities of daily living (ADL) (eleven items). The second subscale relates to instrumental activities of daily living (IADL) (seven items). Items can be answered on a four point scale ranging from 'Yes, I can do it fully independently without any difficulty' to 'No, I cannot do it fully independently; I can only do it with someone's help'. Scores range from 18 to 72 (total scale), from 11 to 44 (ADL subscale) and from 7 to 28 (IADL subscale). Higher scores indicate greater disability in activities of daily living.

Statistical analysis

Firstly, to provide an overview of respondents' background characteristics, descriptive statistics were used. 
Secondly, the reliability was determined from agreement between instruments (Kappa statistic based on proposed cut-off points by original authors) and internal consistency. Cronbach's alpha coefficient was calculated to evaluate internal consistency of items. Cronbach's alpha produces the same result as the KuderRichardson Formula 20 (KR-20), which can be used to assess the internal consistency for dichotomous items. ${ }^{25}$ Furthermore, corrected total-item-correlations were calculated.

Thirdly, to assess the validity, non-parametric tests were used as our data were not normally distributed. If less than $25 \%$ (GFI, TFI, SPQ) or 50\% (GARS)23 of the items were missing, these were imputed by means of case mean substitution. ${ }^{26}$ If more items were missing, persons were excluded from the analysis for the particular scale. The construct validity was assessed using Spearman's rank correlation between the three frailty instruments, as the instruments were assumed to measure the same concept of frailty. Frailty and disability are strongly related concepts, ${ }^{5}$ as frail older people have an increased risk of disability and disability exacerbates frailty. ${ }^{2,3,5}$ Substantial associations between frailty and disability were expected. Therefore, construct validity was also assessed by examining associations between frailty and disability, measured by means of the GARS (Spearman's rank correlation). However, correlations should not be too high, otherwise frailty instruments and the GARS would measure the same concept. Furthermore, frail older people were more likely to be older, female, less educated, and had lower incomes compared to their non-frail counterparts. ${ }^{27}$ Since the distribution of frailty scores was non-normally distributed, Mann-Whitney $U$ and Kruskal-Wallis tests were performed to evaluate differences in the distribution of frailty scores among groups with different background characteristics. ${ }^{28}$ For the dichotomous variable gender (female versus male) the Mann-Whitney $U$ test was applied. For categorical variables with more than two groups (age, education and income), Kruskal-Wallis tests were used to compare the distribution of frailty scores among groups.

All statistical analyses were performed using SPSS for Windows, version 16.0 The level of statistical significance was set at $\mathrm{p}=0.05$ (two-tailed). For post-hoc pairwise comparisons a Bonferroni correction was applied, so all effects are reported at a $p=0.02$ level of significance (two-tailed) in the case of three groups (age education, income)

\section{Results}

Participants

Of the 687 community-dwelling older people ( $\geq 70$ years), $532(77 \%)$ returned the questionnaire. The sample consisted of 311 women (59\%) and 221 men (42\%). In total, $64 \%$ of respondents lived in an urban area (Roermond, Amersfoort), while $36 \%$ lived in a rural area (Roggel). When using the proposed cut-off points, the GFI detected 245 frail cases (46\%). The TFI and the SPQ identified 211 (40\%) and 305 (59\%) frail older people, respectively. The mean age of respondents was 77.2 years with a range of $70-97$ years $(S D=5.5)$. Nearly half of the sample (49\%) had a secondary educational level. The largest proportion of people (42\%) had a net income of more than $€ 1500$ (per month/ per household). An overview of background characteristics is presented in Table 1. The sample is representative for the Dutch population of older people. According to a report of the Netherlands Institute for Social Research ${ }^{29}$ slightly more people aged 75 years and older are female. Older people, especially women, are often less educated and have an average income of about $€ 1500$ (per month/ per household).

The frailty instrument with the greatest number of excluded respondents due to missing values ( $>25 \%$ missing values) was the $\mathrm{SPQ}(\mathrm{n}=8)$. For the $\mathrm{GFI}$ and the TFI, one and two persons, respectively, were excluded due to missing values. On an

Table 1 Characteristics of the participants $(n=532)$.

\begin{tabular}{|c|c|c|c|c|}
\hline & & $\begin{array}{c}\text { Men } \\
(n=221)\end{array}$ & $\begin{array}{l}\text { Women } \\
(n=311)\end{array}$ & $\begin{array}{c}\text { Total } \\
(n=532)\end{array}$ \\
\hline Frail' & $n(\%)$ & & & \\
\hline GFI & & 86 (39) & $159(51)$ & $245(46)$ \\
\hline TFI & & $66(30)$ & $145(47)$ & $211(40)$ \\
\hline$S P Q$ & & $148(68)$ & 157 (53) & 305 (59) \\
\hline $\mathrm{Age}^{2}$ & $n(\%)$ & & & \\
\hline $70-74 \mathrm{yrs}$ & & $91(41)$ & 102 (33) & $193(36)$ \\
\hline $75-79$ yrs & & 78 (35) & $115(37)$ & $193(36)$ \\
\hline$\geq 80 \mathrm{yrs}$ & & $52(24)$ & $94(30)$ & $146(27)$ \\
\hline Education & $n(\%)$ & & & \\
\hline $\begin{array}{l}\text { No Education/ } \\
\text { Primary Education }\end{array}$ & & $62(29)$ & $124(41)$ & $186(36)$ \\
\hline $\begin{array}{l}\text { Secondary } \\
\text { Education }\end{array}$ & & $102(47)$ & $151(50)$ & $253(49)$ \\
\hline $\begin{array}{l}\text { Higher } \\
\text { Education }\end{array}$ & & $53(24)$ & $29(10)$ & $82(16)$ \\
\hline Income & $n(\%)$ & & & \\
\hline$\leq € 900$ & & $34(16)$ & $59(21)$ & $93(19)$ \\
\hline$€ 901$ to $€ 1500$ & & $63(30)$ & $131(46)$ & 194 (39) \\
\hline$\geq € 1501$ & & $114(54)$ & 97 (34) & $211(42)$ \\
\hline
\end{tabular}


item-level the number of missing values ranged from zero to eight (GFI), from zero to twelve (TFI) and from one to ten (SPQ). The average number of missing values per item was 2.4, 5.1 and 5.3 for the GFI, the TFI and the SPQ, respectively.

\section{Reliability}

Cohen's Kappa coefficients between instruments were 0.74 (GFI-TFI), 0.28 (SPQ-GFI) and 0.25 (SPQ-TFI). According to Landis \& Koch ${ }^{30}$ the kappa values indicated good agreement between GFI and TFI and fair agreement between the between GFI and SPQ and TFI and SPQ $(<0.20=$ poor, $0.21-0.40=$ fair, $0.41-0.60=$ moderate, 0.60 $0.80=$ good, $0.81-1.00=$ very good agreement). Cronbach's alpha coefficients for the GFI, the TFI and the SPQ were $a=0.73, a=0.79$ and $a=0.26$, respectively. The higher Cronbach's alpha, the more reliable the test is. Alpha values above 0.70 indicated a satisfactory internal consistency for a scale. ${ }^{31}$ Corrected item-total correlations ranged from 0.14 to 0.55 with a mean of 0.30 (GFI), from 0.18 to 0.58 with a mean of $0.39(\mathrm{TFI})$ and from 0.13 to 0.25 with a mean of 0.18 (SPQ)

Validity

Frailty instruments correlated significantly $(p<0.05)$ with each other and with disability measured by means of the GARS (convergent validity). The association between the GFI and the TFI scores was $r=0.87$. Correlations with the SPQ scores were $r=0.47$ for the GFI and $r=0.42$ for the TFI. The correlation coefficients between frailty instruments and disability (GARS) were $r=0.57$ (GFI-GARS), $r=0.61$ (TFI-GARS) and $r=0.46$ (SPQ- GARS). An overview of all correlation coefficients is presented in Table 2.

Table 2 Spearman correlation coefficients (99\%-confidence interval) among frailty instruments and GARS

\begin{tabular}{|l|c|c|c|c|c|}
\hline & GFI & TFI & SPQ & $\begin{array}{c}\text { GARS } \\
\text { Total scale }\end{array}$ & $\begin{array}{c}\text { GARS } \\
\text { ADL scale }\end{array}$ \\
\hline TFI & $\begin{array}{l}0.87 \\
(0.84-0.89)\end{array}$ & & & & \\
\hline SPQ & 0.47 & 0.42 & & & \\
& $(0.1-0.55)$ & $(0.32-0.51)$ & & & \\
\hline GARS & 0.57 & 0.61 & 0.46 & & \\
\hline Total scale & $(0.49-0.64)$ & $(0.53-0.68)$ & $(0.37-0.54)$ & & \\
\hline GARS & 0.54 & 0.58 & 0.41 & 0.94 & \\
\hline ADL scale & $(0.46-0.61)$ & $(0.5-0.65)$ & $(0.32-0.5)$ & $(0.93-0.95)$ & \\
\hline GARS & 0.55 & 0.57 & 0.46 & 0.96 & 0.79 \\
\hline IADL scale & $(0.47-0.62)$ & $(0.49-0.64)$ & $(0.37-0.54)$ & $(0.95-0.97)$ & $(0.74-0.83)$ \\
\hline
\end{tabular}

Table 3 shows the mean total scores and standard deviations of the GFI, the TFI and the SPQ related to (a) age, (b) sex, (c) education and (d) income. Scores on the GFI and the TFI were significantly higher for females, for persons with a higher age and for persons with lower education and lower incomes as compared to males, persons with a lower age, and persons with higher education and higher incomes. In

Table 3 Mean scores on frailty instruments' according to sex, age, educational level and income.

\begin{tabular}{|c|c|c|c|c|}
\hline & & GFI & TFI & SPQ \\
\hline Total sample & Mean (sd) & $3.6(2.8)$ & $4.2(3.2)$ & $1.9(1.2)$ \\
\hline Sex & Mean (sd) & & & \\
\hline Male & & $3.2(2.7)$ & $3.4(3.1)$ & $2.1(1.2)$ \\
\hline \multirow[t]{2}{*}{ Female } & & $3.9(2.8)$ & $4.7(3.2)$ & $1.7(1.2)$ \\
\hline & $\begin{array}{l}\text { Z statistic } \\
\text { (P-value) }\end{array}$ & $\begin{array}{l}-3.31 \\
(0.001)\end{array}$ & $\begin{array}{l}-4.95 \\
(0.000)\end{array}$ & $\begin{array}{c}-3.28 \\
(0.001)\end{array}$ \\
\hline Age & Mean (sd) & & & \\
\hline$\leq 74 \mathrm{yrs}$ & & $3.0(2.7)$ & $3.3(3.1)$ & $1.7(1.2)$ \\
\hline 75-79 yrs & & $3.6(2.8)$ & $4.2(3.3)$ & $1.9(1.2)$ \\
\hline \multirow[t]{2}{*}{$\geq 80 \mathrm{yrs}$} & & $4.4(2.7)$ & $5.3(3.1)$ & $2.2(1.2)$ \\
\hline & $\begin{array}{l}\text { Chi-square }^{2} \\
\text { (P-value) }\end{array}$ & $\begin{array}{l}27.58 \\
(0.000)\end{array}$ & $\begin{array}{c}37.2 \\
(0.000)\end{array}$ & $\begin{array}{c}15.84 \\
(0.000)\end{array}$ \\
\hline Education & Mean (sd) & & & \\
\hline $\begin{array}{l}\text { No Education/ } \\
\text { Primary Education }\end{array}$ & & $4.1(3.0)$ & $4.7(3.5)$ & $2.0(1.3)$ \\
\hline $\begin{array}{l}\text { Secondary } \\
\text { Education }\end{array}$ & & $3.6(2.7)$ & $4.1(3.1)$ & $1.8(1.2)$ \\
\hline \multirow[t]{2}{*}{$\begin{array}{l}\text { Higher } \\
\text { Education }\end{array}$} & & $2.7(2.2)$ & $3.1(2.8)$ & $1.7(1.0)$ \\
\hline & $\begin{array}{l}\text { Chi-square }^{2} \\
\text { (P-value) }\end{array}$ & $\begin{array}{c}12.13 \\
(0.002)\end{array}$ & $\begin{array}{l}13.47 \\
(0.001)\end{array}$ & $\begin{array}{c}2.47 \\
(0.291)\end{array}$ \\
\hline Income & Mean (sd) & & & \\
\hline$\leq € 900$ & & $4.6(3.0)$ & $5.3(3.6)$ & $2.2(1.4)$ \\
\hline$€ 901$ to $€ 1500$ & & $4.0(2.9)$ & $4.8(3.4)$ & $1.8(1.3)$ \\
\hline \multirow[t]{2}{*}{$\geq € 1501$} & & $2.8(2.3)$ & $3.1(2.6)$ & $1.8(1.0)$ \\
\hline & $\begin{array}{l}\text { Chi-square }^{2} \\
(P \text {-value })\end{array}$ & $\begin{array}{l}29.42 \\
(0.000)\end{array}$ & $\begin{array}{c}37.16 \\
(0.000)\end{array}$ & $\begin{array}{l}5.05 \\
0.080\end{array}$ \\
\hline
\end{tabular}


contrast, on the SPQ we found higher scores among males as compared to females. Scores on the SPQ increased with higher age, lower education and lower incomes, however, the differences with respect to education and income were not significant $(p=0.29$ and $p=0.08$ respectively).

\section{Discussion}

The purpose of the present study was to evaluate and compare the psychometric properties of three screening instruments that define frailty from a multifactorial perspective and which are applicable for postal screening in community-dwelling older people. The chosen instruments were the Groningen Frailty Indicator (GFI), the Tilburg Frailty Indicator (TFI) and the Sherbrooke Postal Questionnaire (SPQ).

From the present study we may conclude that: (1) prevalence estimates of frailty ranged between 40\% (TFI), 46\% (GFI) and 59\% (SPQ); (2) the agreement in identifying frailty between the GFI and the TFI was satisfactory (kappa $=0.74$ ) and the agreements between the SPQ and the GFI and the TFI, respectively, were much lower; (3) both the GFI and the TFI had high internal consistency in contrast to the SPQ; (4) the GFI and the TFI had better construct validity in comparison with the SPQ.

Prevalence estimates of $40 \%$ to $60 \%$ found in the present study can be considered as high. It is important to bear in mind that prevalence estimates strongly depend on the interpretation of the concept of frailty and the approach that is chosen to measure it. ${ }^{32}$ In a recent study by Santos-Eggimann and colleagues, ${ }^{33}$ a distinction was made between frail and pre-frail older people based on the frailty phenotype of Fried and colleagues. ${ }^{5,34}$ In a Dutch sample of community-dwelling older people, SantosEggimann and colleagues $^{33}$ found a frailty prevalence of $11 \%$, while $39 \%$ were identified as pre-frail. These results indicate that the instruments in our study, based on the proposed cut-off points, may identify pre-frail instead of frail older people. Further research is needed to provide a better view on relevant cut-off points for frailty instruments. Longitudinal studies are needed to investigate the predictive power of instruments to identify older people who are at risk for adverse health outcomes in the near future.

Steverink and colleagues ${ }^{16}$ suggested that the GFI is an internally consistent scale with positive indications for construct and clinical validity. The present study supports these findings. Similar results for the TFI may be explained by seven out of fifteen items of the TFI being identical with the GFI. These items are about hearing and vision capacity, unintentional weight loss and psychosocial and cognitive functioning. Scores on the Sherbrooke Postal Questionnaire were higher for males compared with females. This finding is inconsistent with the literature. ${ }^{27}$ However, other findings on the Sherbrooke Questionnaire (higher score with higher age, lower educational level and lower incomes) are well in line with the literature. ${ }^{27}$ Previous studies about the SPQ have reported regarding the predictive validity of the $S P Q, 8,19,20,22$ however, in the present study the SPQ showed less reliability and construct validity. Conclusions about predictive validity cannot be drawn for any of the three instruments.

The findings of the present study should be interpreted in the context of potentia limitations. First, little is known about the test-retest reliability of the instruments. Second, there is no gold standard available as an external criterion of frailty. Future studies could analyse the predictive validity of the frailty instruments with respect to disability, health service utilisation and mortality. Last, the SPQ was not fully used according to the protocol, as non-respondents were excluded from analyses. According to the protocol of the $\mathrm{SPQ}^{8}$, non-respondents should also be considered at risk (which would have resulted in a prevalence estimate of $67 \%$ instead of $59 \%$ ). The strengths of the present study are the comparisons of the psychometric properties of the frailty instruments, the proven feasibility of the postal procedure ${ }^{24}$ and the response rate of $77 \%$, which is as good as, or even better than, previous studies in which postal screening procedures were applied. ${ }^{24,35,36}$

Although most older people may visit their GP regularly, primary care often fails in the identification of the health care needs of older people. ${ }^{37}$ Screening has the potentia to identify older people at risk, followed by comprehensive assessment when needed. ${ }^{7}$ Frailty instruments have to provide reliable and valid answers and have to be feasible. ${ }^{15}$ The psychometric properties of the TFI were slightly better than those of the GFI. However, the number of missing values was lower for GFI items than for TFI items, indicating a higher feasibility of the GFI. Based on these findings it is not yet possible to conclude whether the GFI or the TFI should be preferred for postal screening. The SPQ is less appropriate with regard to its psychometric quality and missing values.

The Frailty Index ${ }^{8,11}$ is a simple measure that is based on self-reports. However less is known about its feasibility for postal screening. Investigating the feasibility and validity of the Frailty Index as a postal screening instrument may be a point of interest for future research. Future (longitudinal) research into the psychometric properties of the GFI and the TFI is urgently needed with regard to predictive validity and test-retest reliability of the GFI and the TFI. In addition, comparing the GFI and the TFI with other frailty-related constructs would lead to more insight into their construct validity.

\section{Conclusion}

Valid screening instruments for identifying community-dwelling frail older people are needed for disability prevention. The GFI and the TFI have shown high interna consistency and construct validity, in contrast to the SPQ. Prevalence estimates of 
frailty ranged from $40 \%$ to $59 \%$. Most agreement was found between the GFI and the $\mathrm{TFI}$. Based on these findings, it is not possible to conclude whether the GFI or the TFF should be preferred for screening. The SPQ seems less appropriate. Further research is needed.

\section{Acknowledgements}

The authors are grateful to the patients for their willingness to participate in the present study. The Centre for Data and Information Management (MEMIC) Maastricht University is acknowledged for their assistance in the data collection. This research has been funded by Zuyd University of Applied Sciences.

\section{References}

1. Slaets JPJ. Vulnerability in the elderly: frailty. Med Clin North Am. 2006;90(4):593-60

Levers MJ, Estabrooks CA, Ross Kerr JC. Factors contributing to frailty: literature review. J Adv Nurs. 2006;56(3):282-91.

3. Markle-Reid M, Browne G. Conceptualizations of frailty in relation to older adults. J Adv Nurs 2003:44(1):58-68.

4. Pel Littel RE, Schuurmans MJ, Emmelot Vonk MH, Verhaar HJJ. Frailty: defining and measuring of a concept. J Nutr Health Aging. 2009;13(4):390-4.

5. Fried LP, Ferrucci L, Darer J, Williamson JD, Anderson G. Untangling the concepts of disability, frailty, and comorbidity: implications for improved targeting and care. Gerontol A Biol Sci Med Sci 2004;59(3):255-63.

6. Daniels R, Metzelthin S, Van Rossum E, De Witte L, Van den Heuvel W. Interventions to prevent disability in frail community-dwelling older persons: an overview Eur J Ageing 2010;7(1):37-55

7. De Lepeleire J, Degryse J, Iliffe S, Mann E, Buntinx F. Family physicians need easy instruments for frailty. Age Ageing. 2008;37(4):484; author reply -5.

8. Hebert R, Bravo G, Korner-Bitensky N, Voyer L. Predictive validity of a postal questionnaire for screening community-dwelling elderly individuals at risk of functional decline. Age Ageing. 1996;25(2):159-67.

9. Daniels R, Van Rossum HIJ, De Witte LP, Van den Heuvel WJA. Frailty in older age: concepts and relevance for occupational and physical therapy. Phys Occup Ther Geriatr. 2008;27(2):81-95.

10. Bergman $\mathrm{H}$, Beland $F$, Karunanthan $S$, Hummel S, Hogan D, Wolfson C. Developing a working framework

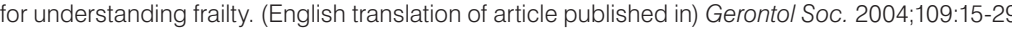
2007,02(7), $722-7$.

. Seanle SD, Mitnitski A, Gahbauer EA, Gill TM, Rockwood K. A standard procedure for creating a frailty index. BMC Geriatr. 2008:8:24

13. Guralnik JM, Branch LG, Cummings $S R$, Curb JD. Physical performance measures in aging research. $J$ Gerontol. 1989;44(5):M141-6.

14. Kempen GIJM, van Heuvelen MJG, van den Brink RHS, Kooijman AC, Klein M, Houx PJ, Ormel J. Factors affecting contrasting results between self-reported and performance-based levels of physical limitation Age Ageing. 1996;25(6):458-6

15. Martin FC, Brighton P. Frailty: different tools for different purposes? Age Ageing. 2008;37(2):129-31.

6. Steverink N, Slaets JPJ, Schuurmans H, van Lis M. Measuring Frailty: development and testing of the Groningen Frailty Indicator (GFI). Gerontologist. 2001;41(1):236-7.

7. Gobbens RJJ, van Assen MALM, Luijkx KG, Wijnen-Sponselee MT, Schols JMGA. The Tilburg Frailty Indicator: Psychometric Properties. J Am Med Dir Assoc. 2010;11(5):344-55.

8. Frieswijk N, Steverink N, Buunk BP, Slaets JPJ. The effectiveness of a bibliotherapy in increasing the selfmanagement ability of slightly to moderately frail older people. Patient Educ Couns. 2006;61(2):219-27.

19. Hemsley Z, Roberts H, Thomas G, Aihie Sayer A, Gove I, Turner G, Meakins P, Morran-Ryan M, Purcel A, Powell J. Can the Sherbrooke postal questionnaire predict who will require comprehensive assessmen in the single assessment process? Age Ageing. 2005;34 (Suppl2):ii20

20. Roberts HC, Hemsley ZM, Thomas G, Meakins P, Powell J, Robison J, Gove I, Turner G, Sayer AA. Nurse-led implementation of the single assessment process in primary care: a descriptive feasibility study. Age Ageing. 2006;35(4):394-8.

21. Schuurmans $\mathrm{H}$, Steverink $\mathrm{N}$, Lindenberg S, Frieswijk $\mathrm{N}$, Slaets JPJ. Old or frail: what tells us more? Gerontol A BIO/ SCI Med SCl. 2004;59(9):M962-5.

22. Walker $L$, Jamrozik K, Wingfield $D$. The Sherbrooke Questionnaire predicts use of emergency services, Age Ageing. 2005;34(3):233-7.

23. Kempen GIJM, Miedema I, Ormel J, Molenaar W. The assessment of disability with the Groningen Activity Restriction Scale. Conceptual framework and psychometric properties. Soc Sci Med. 1996;43(11):1601-10. 
24. Steinbusch C. Screening of frail elderly in the community. The feasibility and psychometric properties of three instruments [MSc thesis]. Maastricht: School for Public Health and Primary Care, Maastricht; 2008. 25. Kuder GF, Richardson MW. The theory of the estimation of test reliability. Psychometrika. 1937;2:151-60. 26. Fox-Wasylyshyn SM, El-Masri, M.M. Handling missing data in self-report measures. Res Nurs Health. 2005;28 488-95.

27. Avila-Funes JA, Helmer C, Amieva H, Barberger-Gateau P, Le Goff M, Ritchie K, Portet F, Carriere I, Tavernier B, Gutierrez-Robledo LM, Dartigues JF. Frailty among community-dwelling elderly people in France: the three-city study. J Gerontol A Biol Sci Med Sci. 2008;63(10):1089-96

28. Rosner B. Fundamentals of Biostatistics. 6th ed. Duxbury: Thomson Brooks/Cole; 2006.

29. Lokale en nationale monitor gezondheid. 2008; retrieved from http://www.monitorgezondheid.n//home

xml. 0. Landis JR, Koch

31. Nunnally JC. Psychometric theory 2nd edition ed. New York: McGraw-Hill; 1978.

32. Bergman H, Ferrucci L, Guranik J, Hogan DB, Hum S, Karum emerging research and clinical paradigm - issues and controversies. J Gerontol A Biol Sci Med Sci 2007;62(7):731-7.

33. Santos-Eggimann B, Cuenoud P, Spagnoli J, Junod J. Prevalence of frailty in middle-aged and older community-dwelling Europeans living in 10 countries. J Gerontol A Biol Sci Med Sci. 2009;64(6):675-81,

34. Fried LP, Tangen CM, Walston J, Newman AB, Hirsch C, Gottdiener J, Seeman T, Tracy R, Kop WJ, Burke G, McBurnie MA. Frailty in older adults endence for a phenotype. J Gerontol A Biol Sci Med Sci. 2001,56(3).M146-56.

5. Bouman AIE. Home visiting program for older persons with poor health status [PhD thesis]. Maastricht: School for Public Health and Primary Care Maastricht University; 2008.

6. Zijstra GAR. Managing concerns about talls. Fear or laling and avoldance of activity in older people. [PhD thesis]. Masustich. School For Public Health and Primary Care, Maastricht University; 2008.

37. Bowns I, Challis D, Tong MS. Case finding in elderly people: validation of a postal questionnaire. BrJ Gen Pract. 1991;41(344):100-4. 
Interventions to prevent

disability in community-dwelling frail older people: an overview

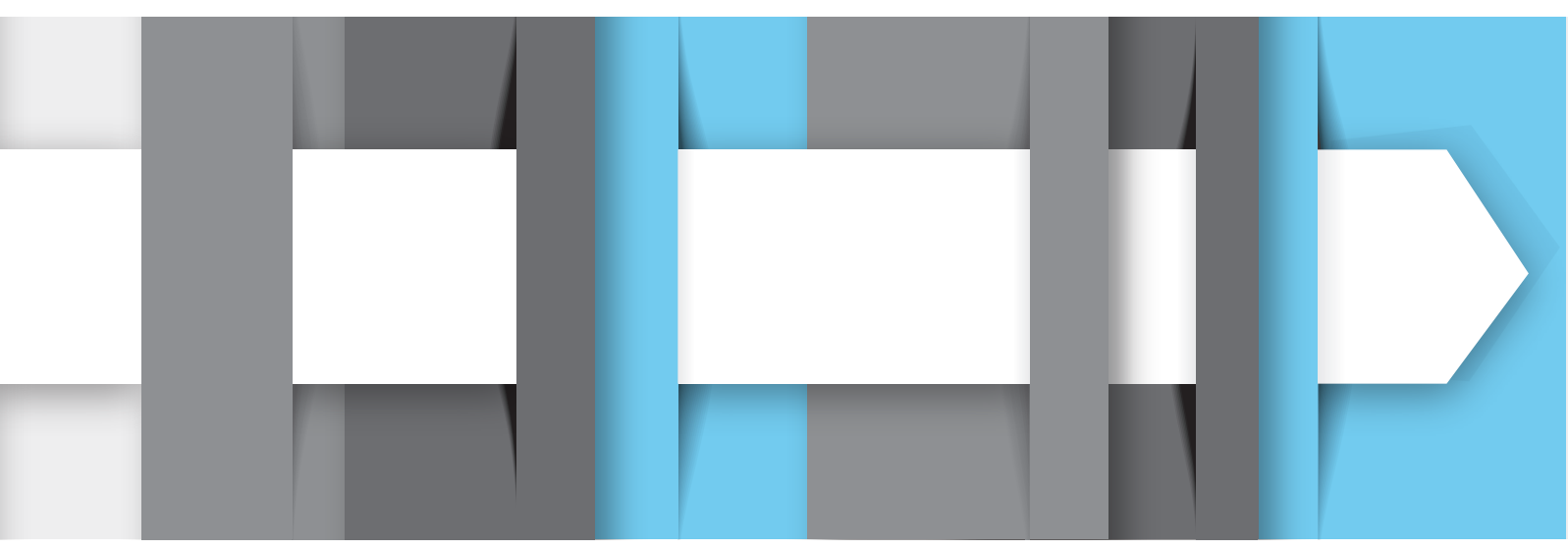

This chapter was published as:

Daniëls, R, Metzelthin, S, van Rossum, E, de Witte, LP, van den Heuvel, W European Journal of Ageing 2010;7:37-55 


\section{Abstract}

This narrative review was conducted to provide an overview of the variety of interventions aimed at disability prevention in community-dwelling frail older people and to summarise promising elements. The search strategy and selection process found 48 papers that met the inclusion criteria. The 49 interventions described in these 48 papers were categorised into 'comprehensive geriatric assessment', 'physical exercise', 'nutrition', 'technology' and 'other interventions'. There is a large diversity within and between the groups of interventions in terms of content, disciplines involved, duration, intensity and setting. For 18 of the 49 interventions, significant positive effects for disability were reported for the experimental group. Promising features of interventions seem to be: multidisciplinary and multifactorial, individualised assessment and intervention, case management, long-term follow-up, physical exercise component (for moderate physically frail older people) and the use of technology. Future intervention studies could combine these elements and consider the addition of new elements.

\section{Introduction}

Frail older people are at much higher risk of disability, hospitalisation, institutionalisation, and death, compared with their age-matched non-frail counterparts. ${ }^{1-3}$ In scenarios that predict future health service delivery in the Western world, the rapid increase in fral older people is seen as one of the major challenges to healthcare. ${ }^{4-6}$ There has been an exponential rise in the use of the term 'frailty' in the literature. ${ }^{7}$ Markle-Reid and Brown (2003) reported substantial disagreement in the literature how frailty is defined and measured. ${ }^{5}$ The debate has focused on whether the frailty should be defined purely in terms of biomedical factors or whether psychosocial factors should be included as well. ${ }^{8}$ From their literature reviews, Levers and colleagues (2006) as well as Aminzadeh and colleagues (2002) conclude that most definitions of frailty do include the idea of loss of age-related reserve capacity, though differences exist regarding other factors contributing to frailty. ${ }^{9,10}$ Despite a lack of consensus about the definition of frailty, a growing number of intervention studies for frail older people are reported, implying that interventions can be targeted at frail older people independent of specific diseases Disability, defined as experienced difficulty in performing activities in any domain of life, ${ }^{11}$ is generally considered as one of the major adverse outcomes of frailty. Prevention of disability in frail older people is seen as a priority for research in geriatrics and can lead to the maintenance of quality of life and reduced healthcare costs.12,13 Severa systematic reviews are available, which focus on specific categories of interventions for frail older people, e.g. comprehensive geriatric assessment, after-care or respite care. ${ }^{14-16}$ No overview is available, however, which provides an extensive overview of the content of the full range of existing programmes for community-living frail older people that are aimed at the prevention of disability. The present study is a narrative review covering a wide range of programmes for community-dwelling frail older people. The primary aim of this study is to provide an overview of the type of interventions studied in randomised or controlled clinical trials regardless of other aspects of thei methodological quality. In order to develop future effective interventions aimed at disability prevention lessons can be learned from such studies. Therefore, the secondary aim of the review is to summarise promising components for future interventions from studies that reported significant effects.

\section{Methods}

\section{Search strategy}

On 3 March 2008, the databases PubMed, the Cochrane Central Register of Controlled Trials (CENTRAL) and CINAHL were searched for randomised controlled clinical trials by use of the words 'frail*', 'vulnerable', 'at risk', 'high risk', 'low functioning', 
and the MESH terms 'chronic disease' and 'disabled persons' in combination with the MESH term 'aged'. Search terms for outcomes focused on disability measures and included terms like 'disabit*', 'functional decline', 'functional capabilit', 'functional performance', 'independen*' and MESH terms 'activities of daily living', 'quality of life' and 'well-being'. In order to restrict the search to interventions that targeted communitydwelling older people, terms like 'home*', 'in-home*', 'communit', 'independent living' and MESH term 'primary care' were added. Additionally, studies were identified by a manual search of reference lists from relevant papers. The search was restricted to articles in English, Dutch and German. There was no restriction on type of intervention or year of publication.

Selection criteria

Inclusion criteria were set for study population, outcome measure, and design. Randomised and controlled clinical trials specifically aimed at community-dwelling frail older people were included. No restrictions were set concerning the definition of frailty. As frailty points to an increased risk of adverse outcomes, only studies that specified the criteria used to operationalise the increased risk were included. Studies that used physical markers to include participants were included as well as studies that used a combination of factors (multifactorial perspective on frailty) as inclusion criteria. Exclusion criteria for the population concerned the selection of participants solely based on age, age and fall incident(s), and age and having one chronic disease.

Disability was used as the outcome measure (regardless of whether it was used as a primary or secondary outcome) and defined as difficulty experienced in performing activities. ${ }^{11}$ Avlund (2004) found that most current studies of disability among older people focus on the ability to carry out activities of daily living. ${ }^{17}$ In this review, studies reporting measurements of Activities of Daily Living (ADL) or Instrumental Activities of Daily Living (IADL) were included.

\section{Data extraction and analysis}

A first selection of relevant studies was made by RD on title-level with a conservative approach, meaning that in case of doubt an article would always be screened on abstract-level. The second (abstract-level) and third selection phase (full-text level) were independently undertaken by two reviewers (RD and SM) scoring 'relevant', 'doubt' or 'irrelevant' on forms. In case of inconsistencies, the reviewers discussed their scores. Consensus on 'irrelevant' led to the exclusion of an article. On severa occasions, the reviewers asked for the involvement of a third party (EvR) to reach consensus.

The same two reviewers performed the data extraction with respect to the aims, target population, design, care disciplines involved, and content of the interventions. Furthermore, follow-up and reported effectiveness on disability was retrieved from the articles. Assessment of the methodological quality of studies was not performed as the primary aim was to provide an overview of the type of interventions reported for community-dwelling frail older people. The research team (RD, SM, EvR, LdW $\mathrm{WvdH}$ ) discussed ways of categorising the studies based on descriptions common in geriatric literature. As this review intends to provide an overview of the content of interventions, it was decided to categorise the interventions according to thei intervention characteristics. Interventions were classified into 'comprehensive geriatric assessment (CGA)', 'physical exercise', 'nutrition', 'assistive technology', and 'other interventions'. Studies that reported significant effects in favour of the experimental group on ADL or IADL measures were further explored (by RD and SM) to identify intervention elements that might explain successful outcomes.

\section{Results}

Four thousand, six hundred and forty-five articles were identified in the literature search. After screening of the titles, 170 studies were considered relevant for further screening on abstract-level. Of these, another 63 studies were excluded, because they did not meet the inclusion criteria (Figure 1). In the next phase, the screening of 107 full-text articles resulted in the exclusion of 59 studies. Of these, 21 were excluded as they did not meet the criteria for population characteristics; Fourteen did not meet the criteria for the outcome measure (disability); and fourteen failed owing to study design. Forty-eight studies, describing 49 interventions, were included. Among these, 26 interventions were categorised as 'comprehensive geriatric assessment (CGA)', twelve as 'physical exercise', three as 'nutrition', two as 'assistive technology' and six were classified as 'other interventions'. All studies were published between 1986 and 2008. There is a large variation in the criteria that studies used to include frail older people (see Table 1). Physical frailty markers are more common as inclusion criteria in physical exercise programmes, while more complex interventions (CGA) generally use a combination of factors, taking a multifactorial perspective on frailty. All 48 studies met the inclusion criterion to measure disability by using measurements on ADL or IADL. However, disability was not the primary outcome measure for all studies. Eleven studies did perform a long-term follow-up measurement ( $\geq 6$ months after the end of the intervention). For nine studies information on follow-up was lacking. 


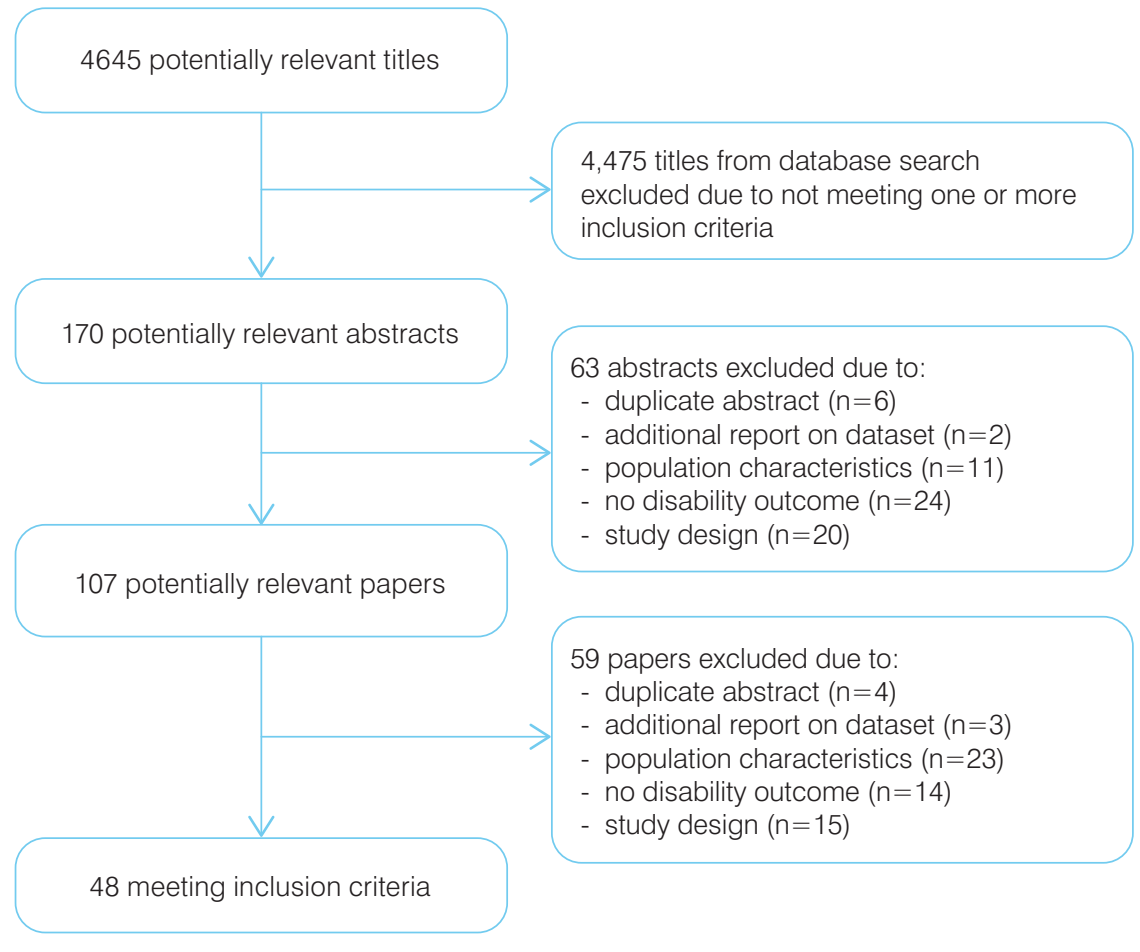

Figure 1 Progress of search for relevant trials.

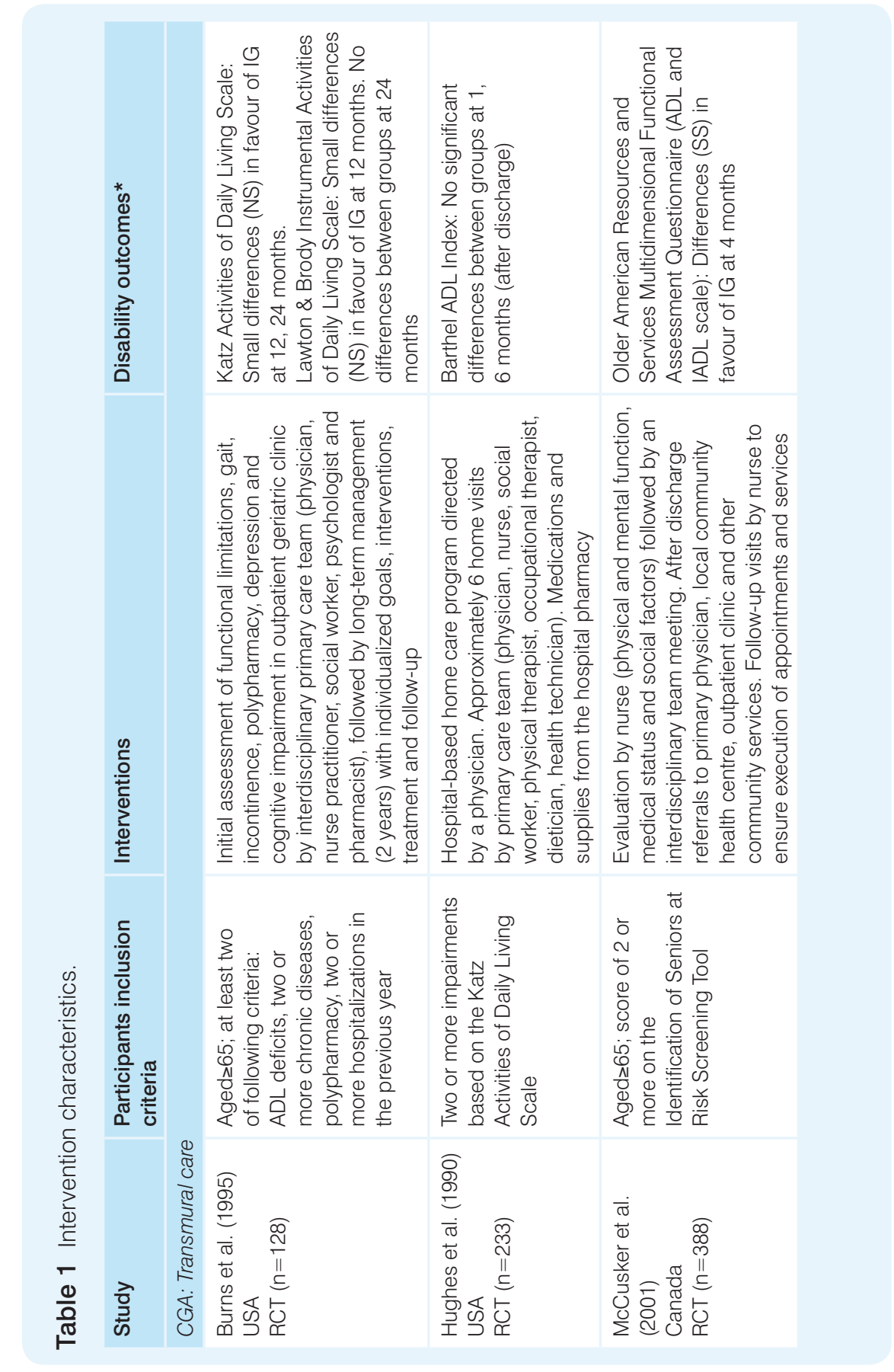




\begin{tabular}{|c|c|c|c|c|c|c|c|}
\hline 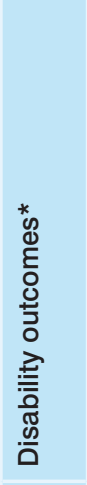 & 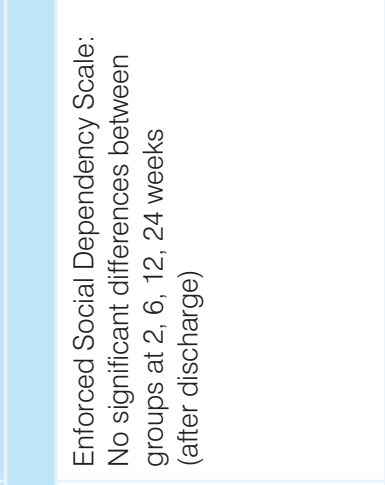 & 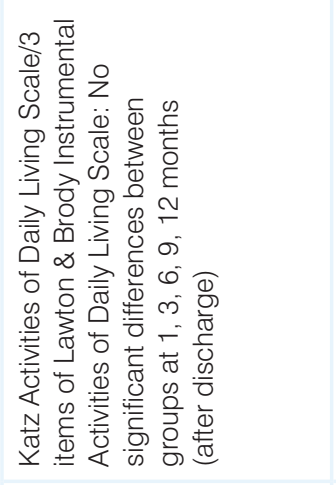 & 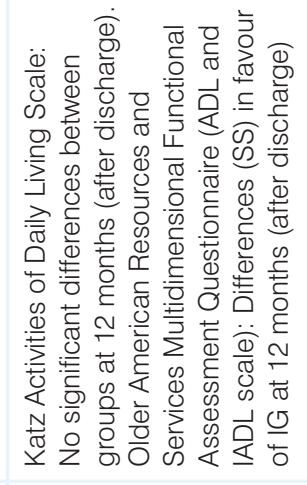 & 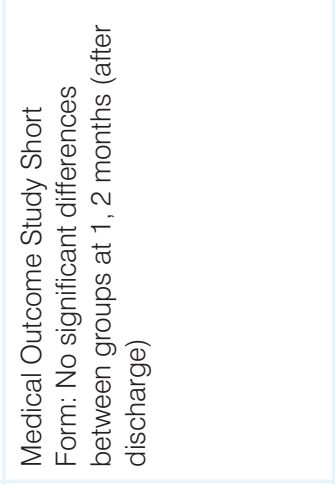 & & 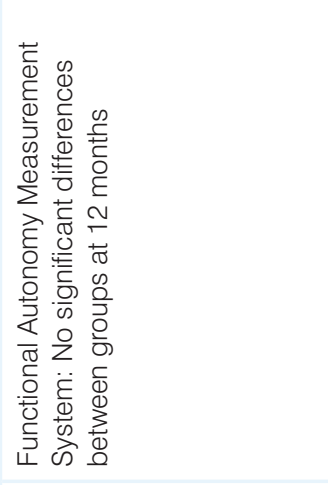 & 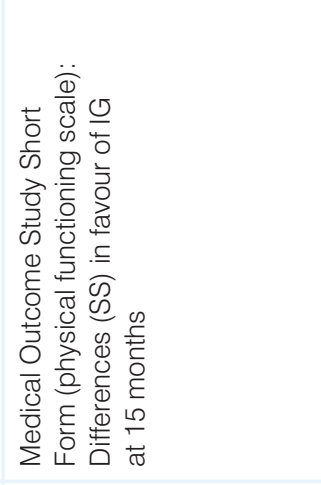 \\
\hline 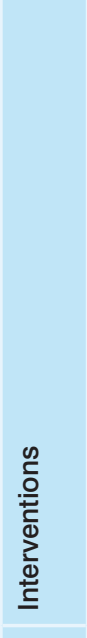 & 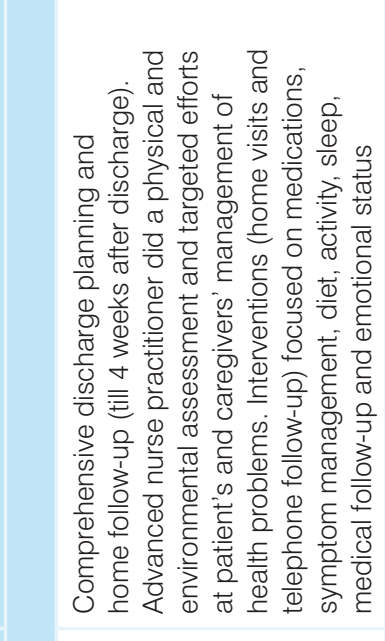 & 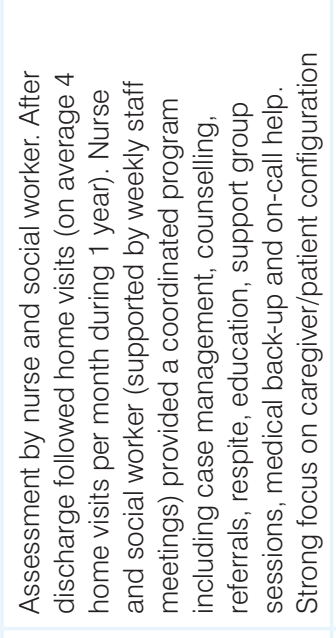 & 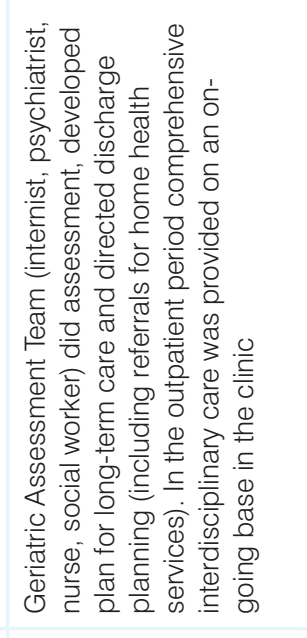 & 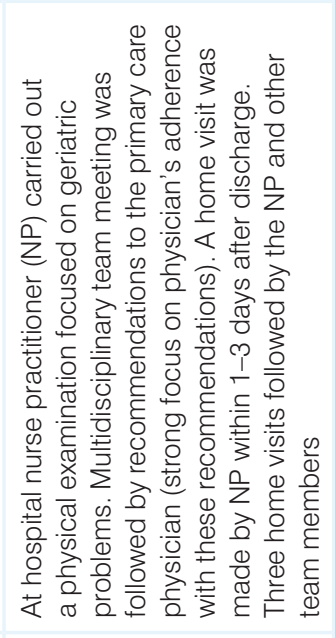 & 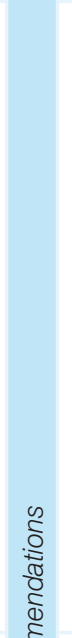 & 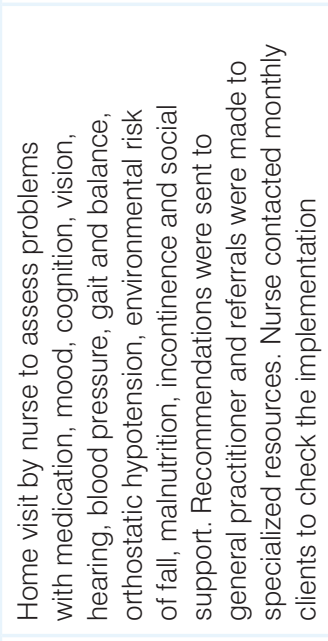 & 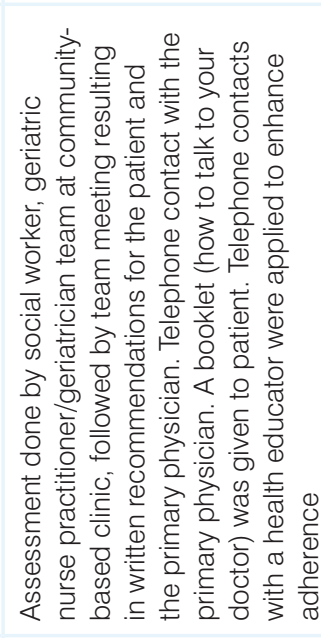 \\
\hline 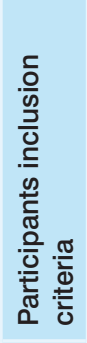 & 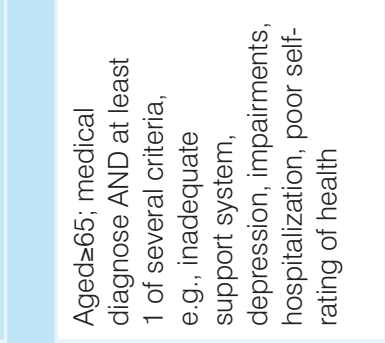 & 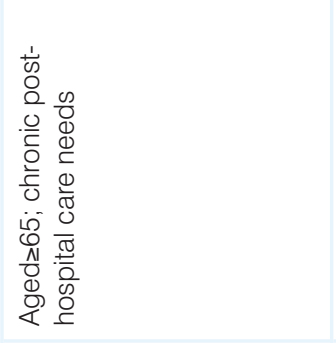 & 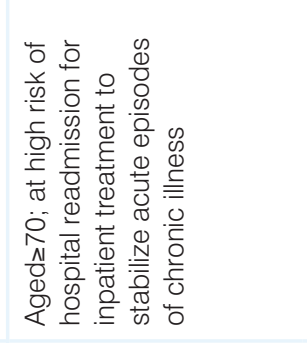 & 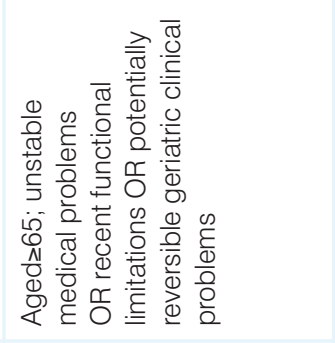 & 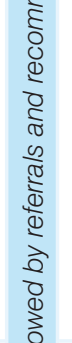 & 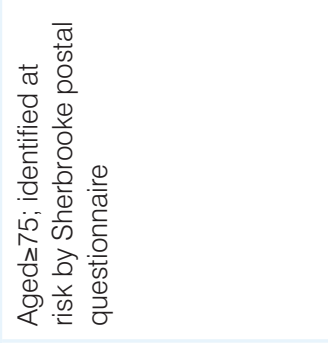 & 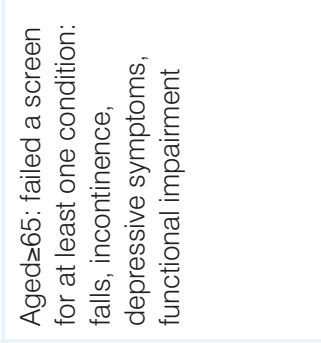 \\
\hline 疍 & 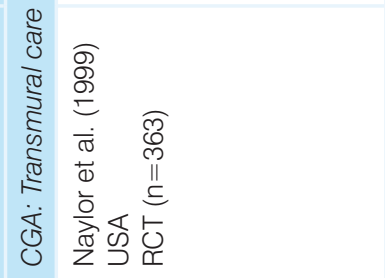 & 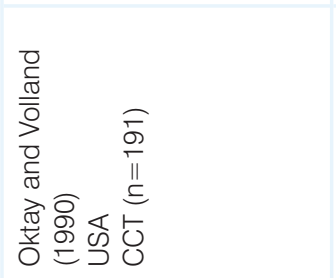 & 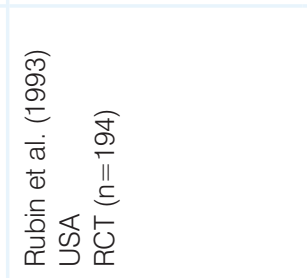 & 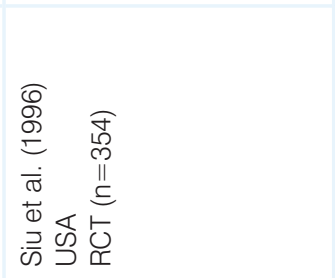 & 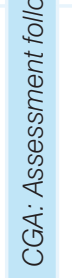 & 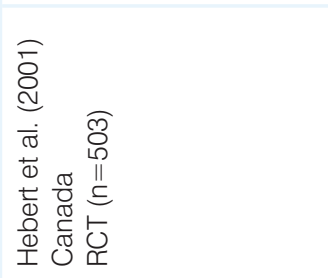 & 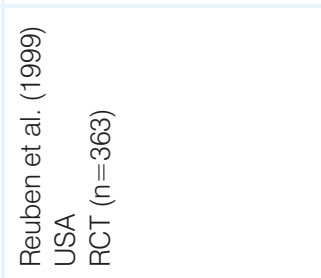 \\
\hline
\end{tabular}




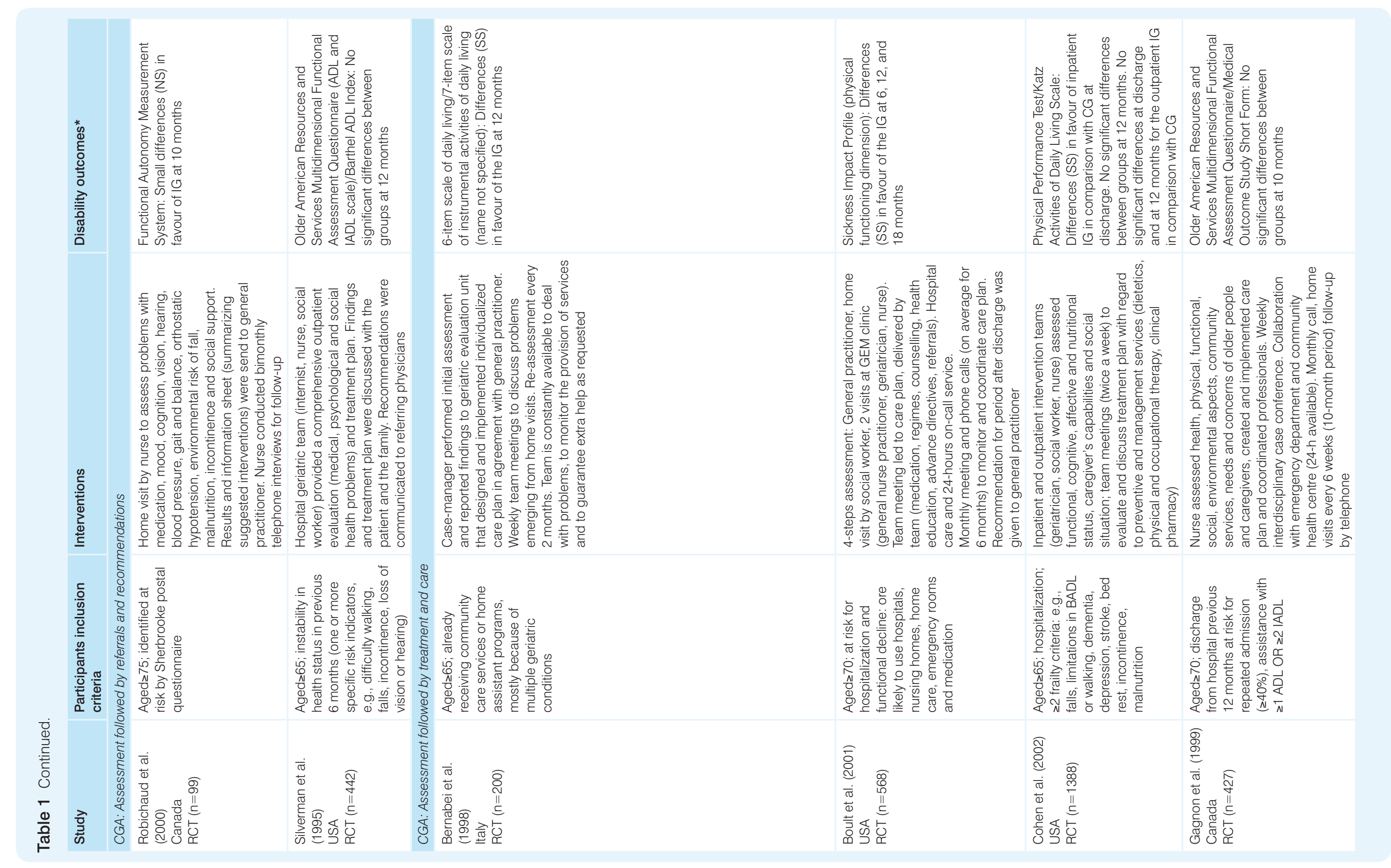




\begin{tabular}{|c|c|c|c|c|c|c|}
\hline & 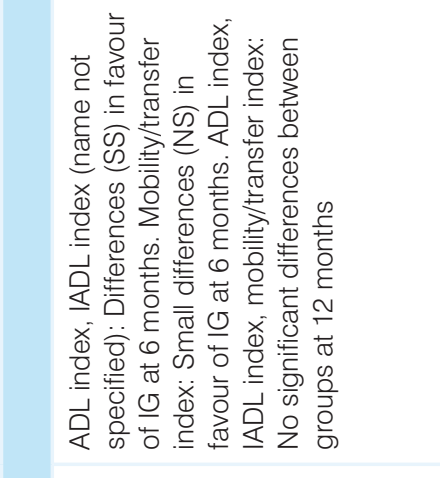 & 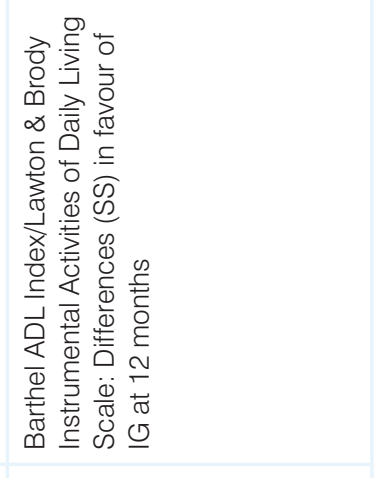 & 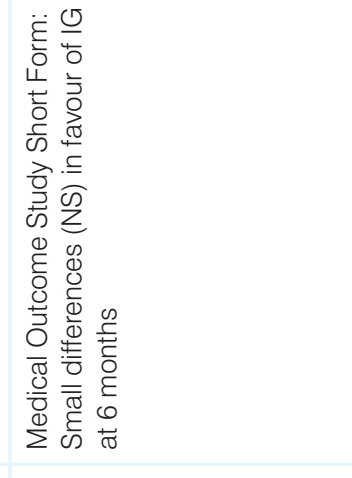 & 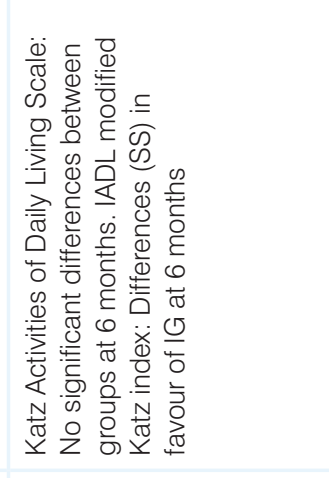 & 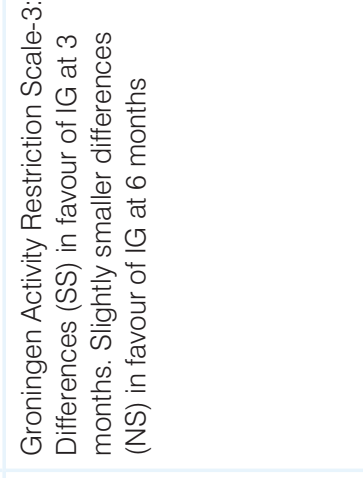 & 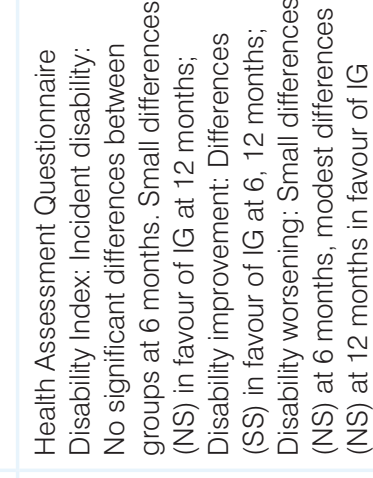 \\
\hline & 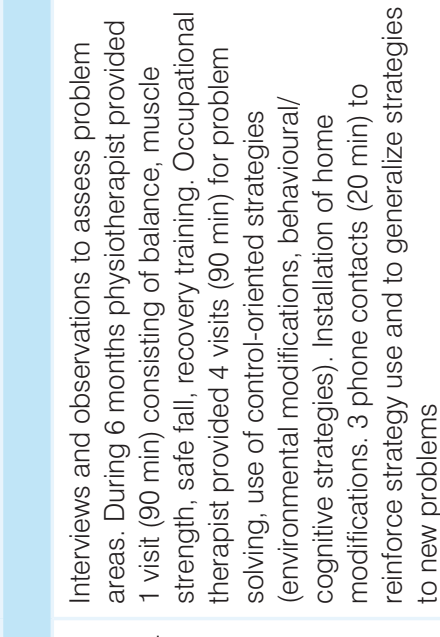 & 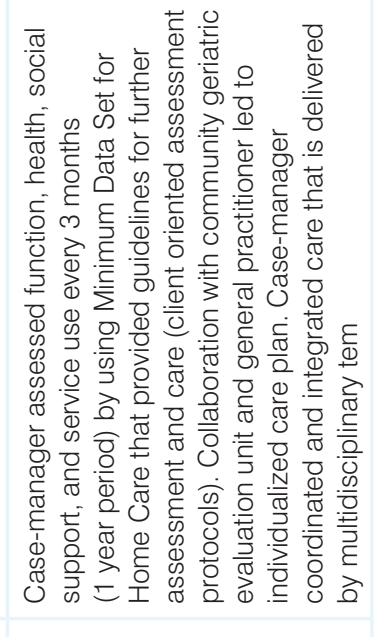 & 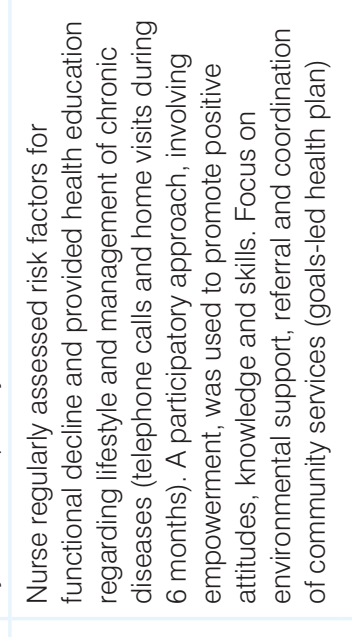 & 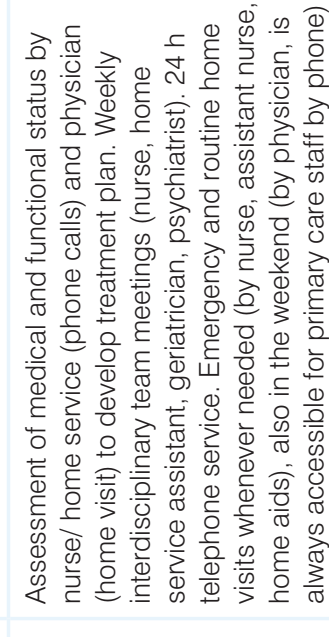 & 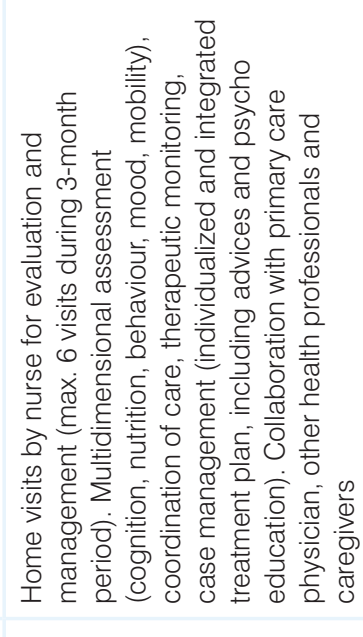 & 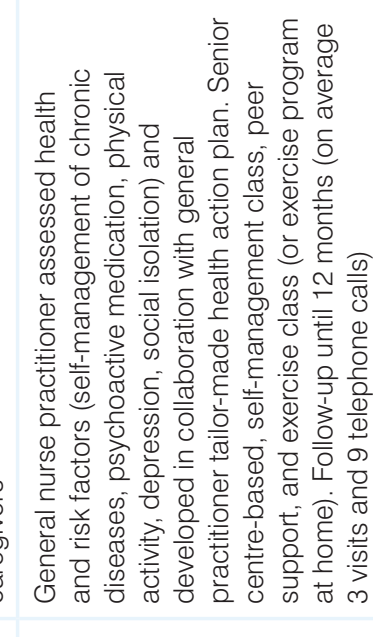 \\
\hline & 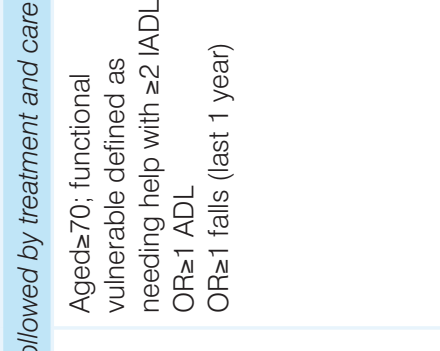 & 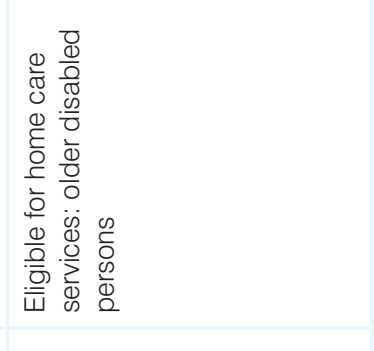 & 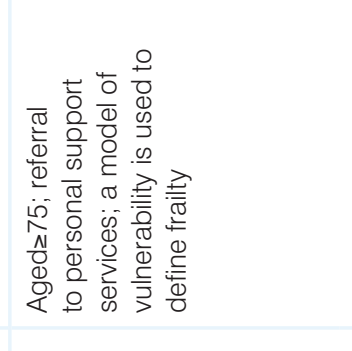 & 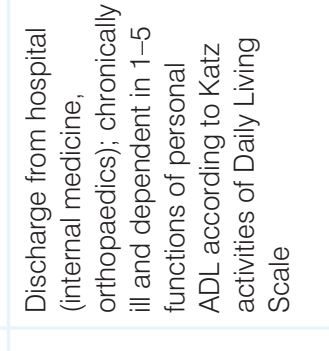 & 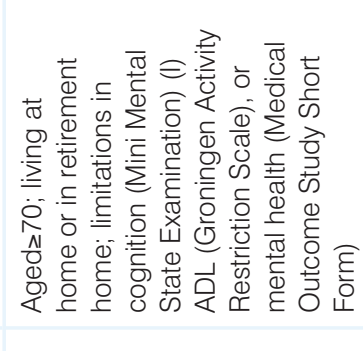 & 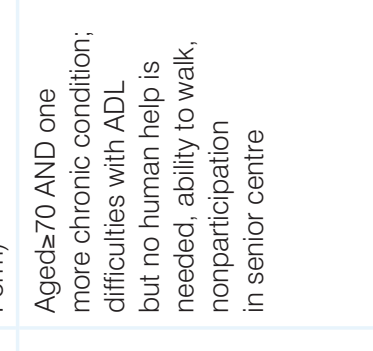 \\
\hline & 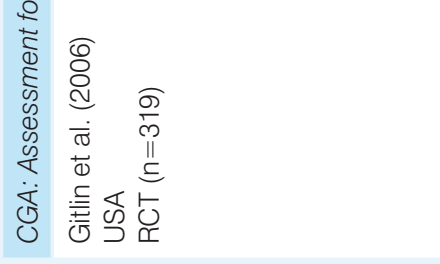 & 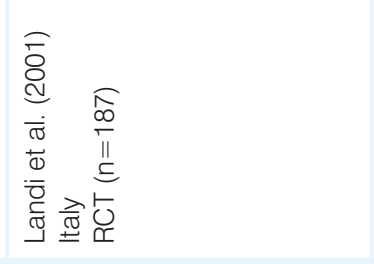 & 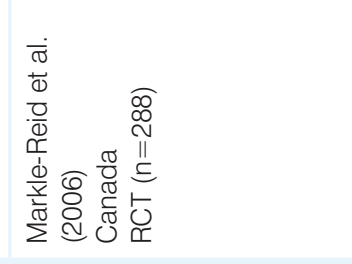 & 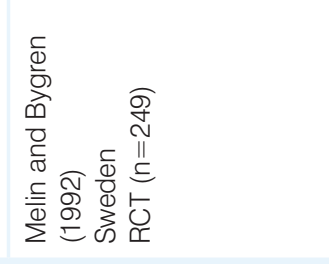 & 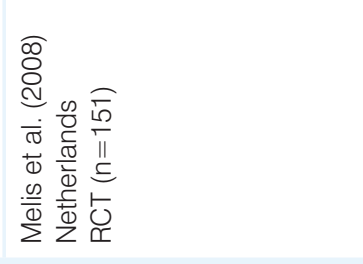 & 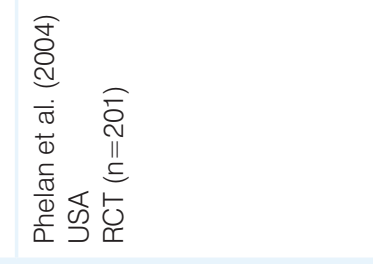 \\
\hline
\end{tabular}




\begin{tabular}{|c|c|c|c|c|c|c|c|c|c|}
\hline 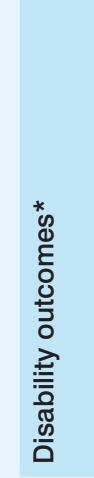 & 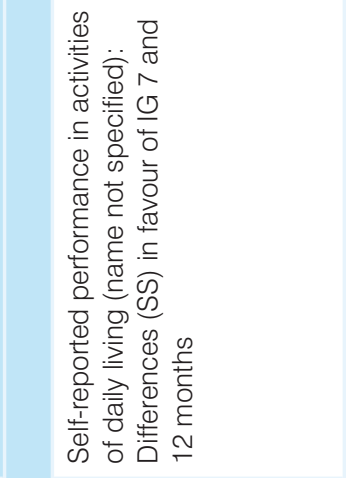 & 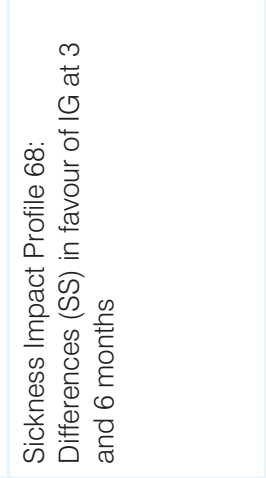 & 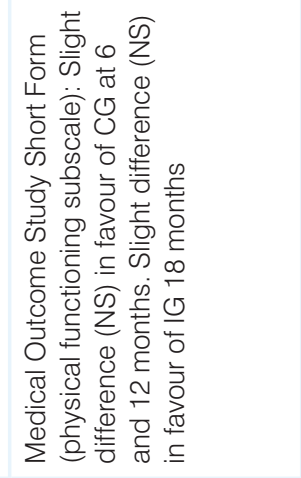 & 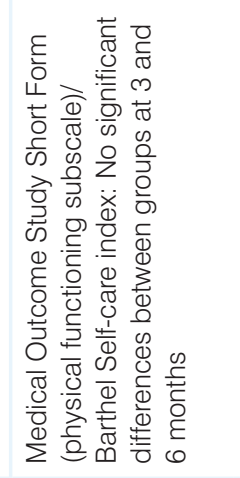 & 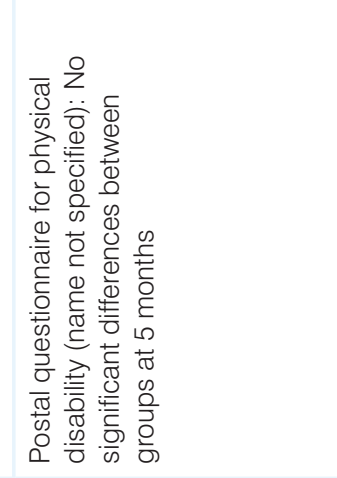 & 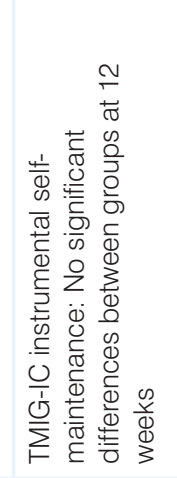 & 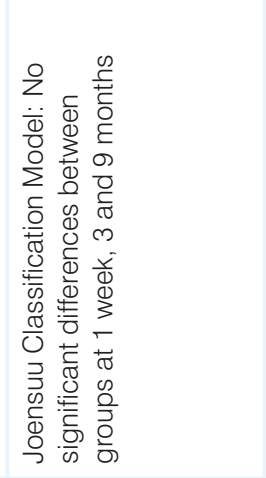 & 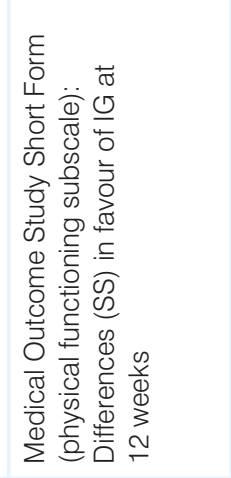 & 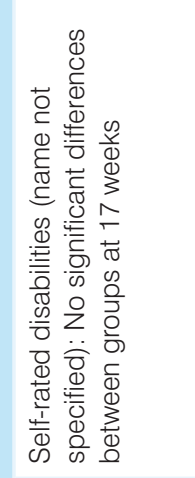 \\
\hline & 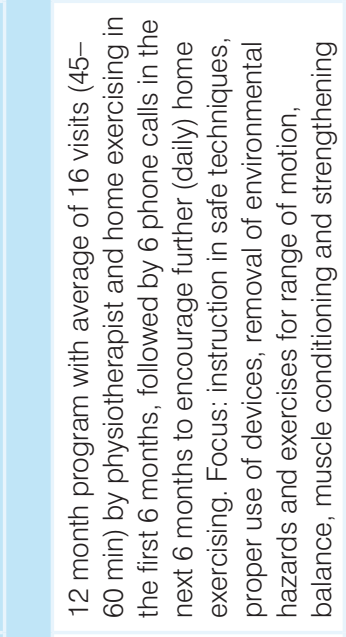 & 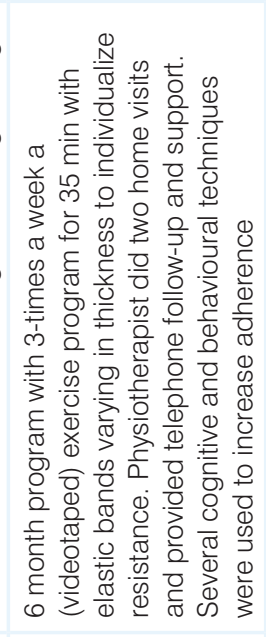 & 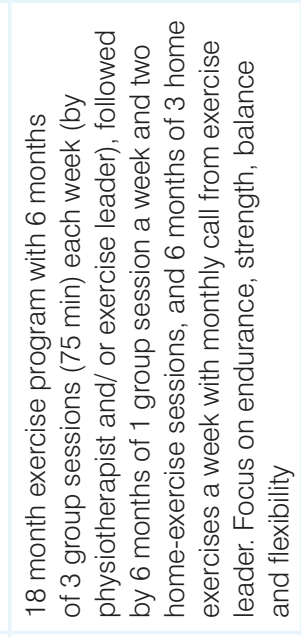 & 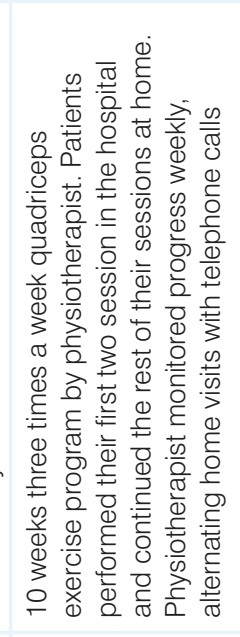 & 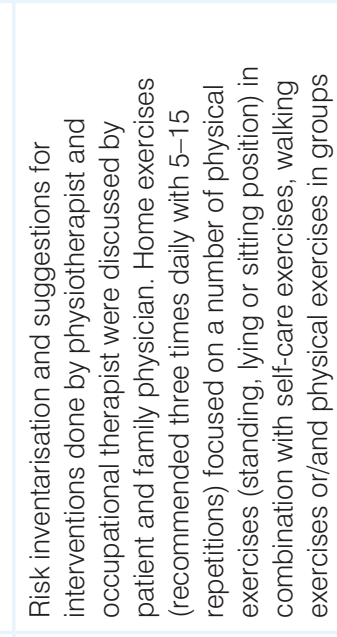 & 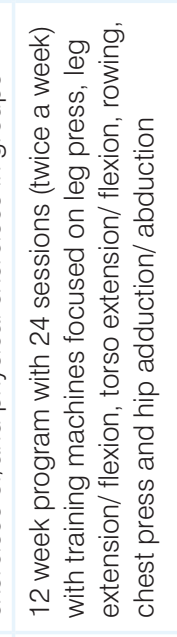 & 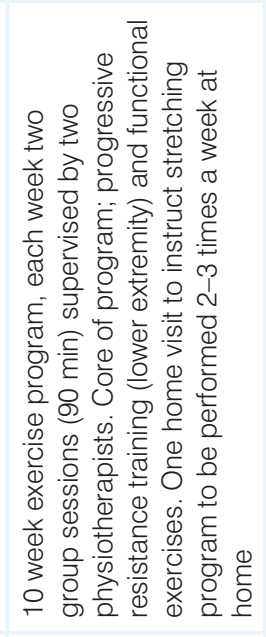 & 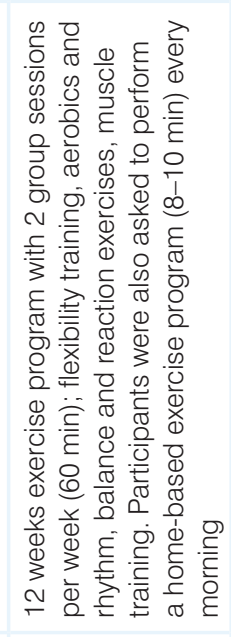 & 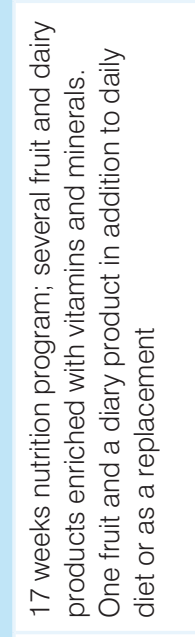 \\
\hline 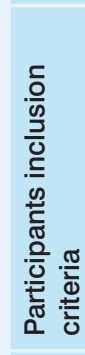 & 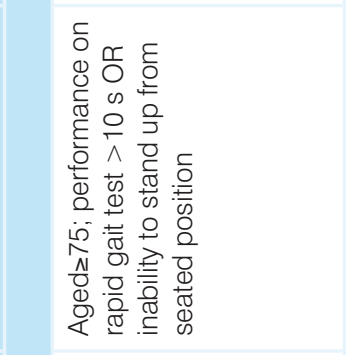 & 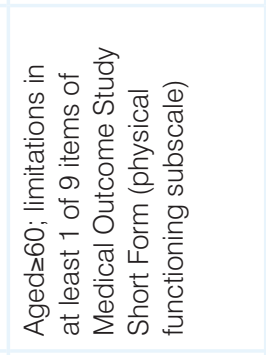 & 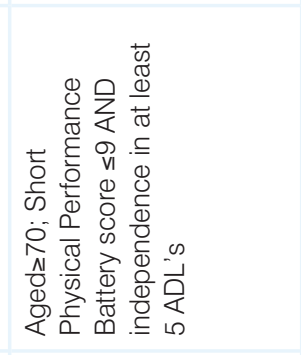 & 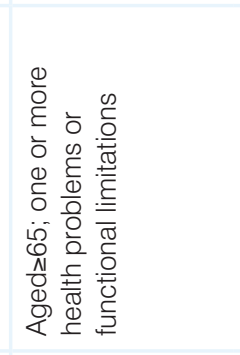 & 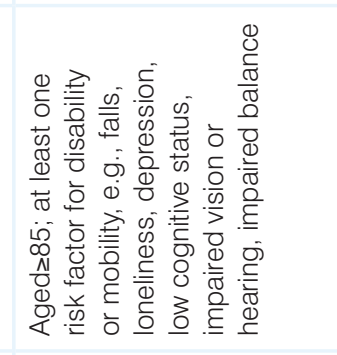 & 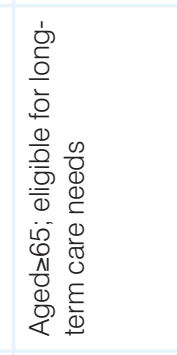 & 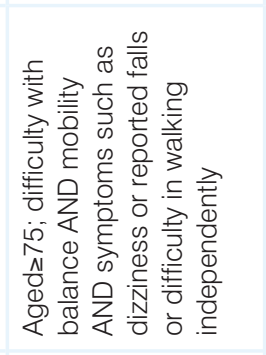 & 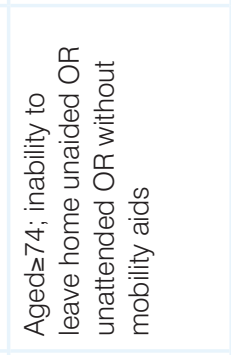 & 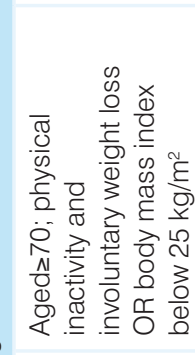 \\
\hline 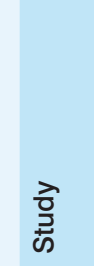 & 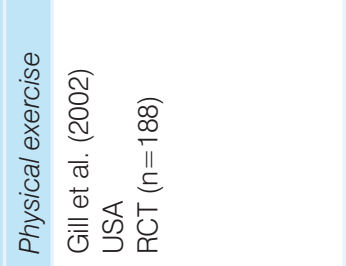 & 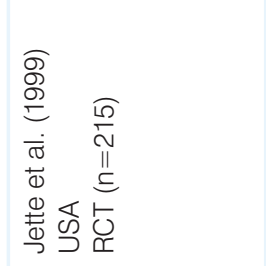 & 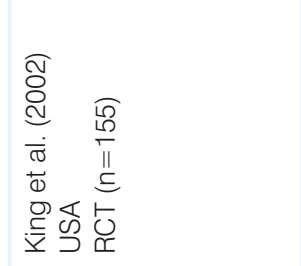 & 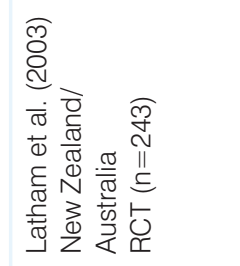 & 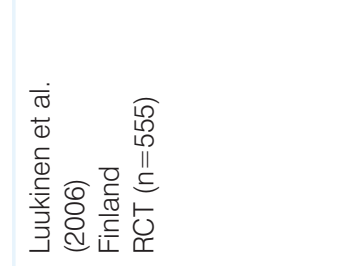 & 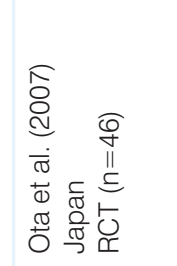 & 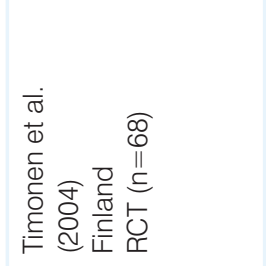 & 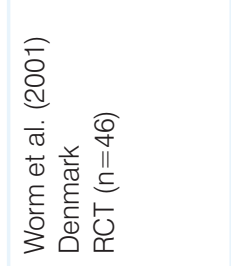 & 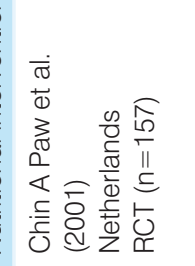 \\
\hline
\end{tabular}




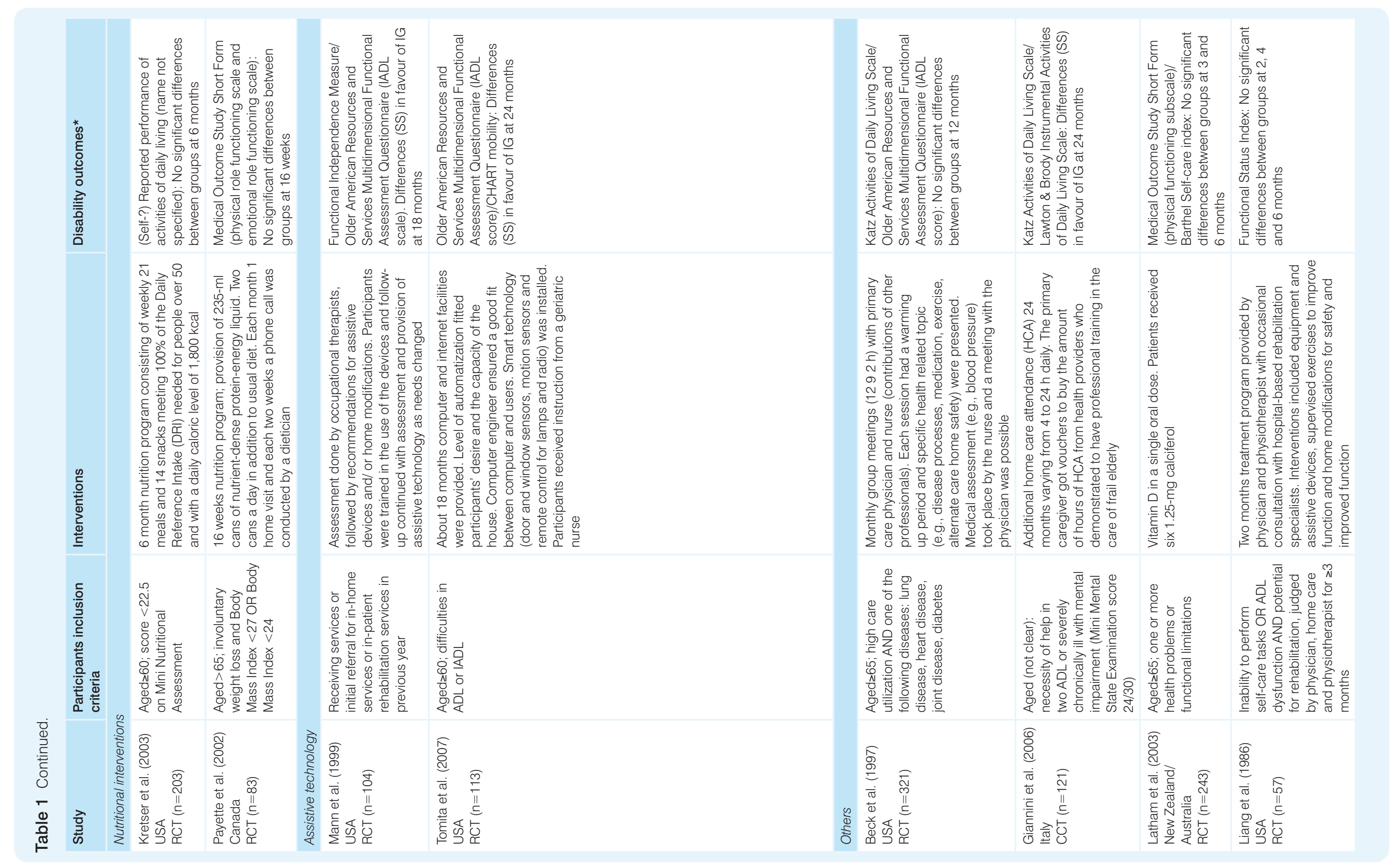




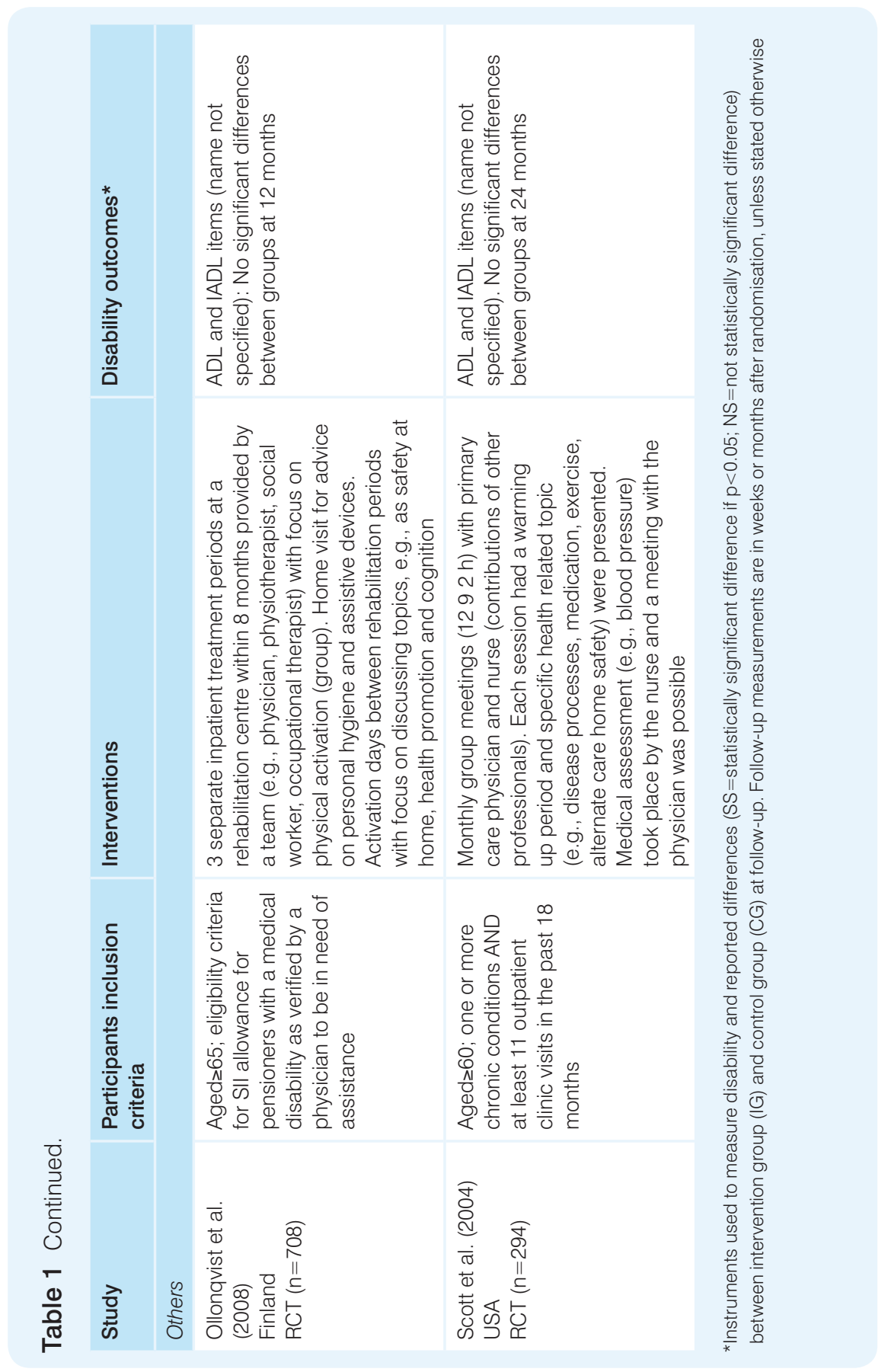

Intervention characteristics

Comprehensive Geriatric Assessment ( $n=26)$

Comprehensive geriatric assessment (CGA) has been defined as 'a multidimensional, often interdisciplinary, diagnostic process intended to determine a frail older persons' medical, psychosocial, and functional capabilities and problems, with the objective of developing an overall plan for treatment and long-term follow-up.'18 For this review the included CGA studies were further divided into transmural care and communitybased care. In the latter, a distinction was made between studies in which assessment was followed by referrals or recommendations and studies where assessment was directly followed by treatment and care.

Transmural Care $(n=7)$

In this review, transmural care points to interventions in which clients were identified and assessed during admission to the hospital setting. After discharge, client referrals were made and interventions were delivered in the community. In the seven studies, assessment was performed by a nurse practitioner ${ }^{19-21}$, a physician ${ }^{22}$ or an interdisciplinary team ${ }^{23-26}$ focusing on a variety of physical, mental, medical and social factors. Usually, the assessment was followed by a team meeting leading to an individualised treatment plan and a variety of actions e.g. referrals ${ }^{19}$ and recommendations to the general practitioner ${ }^{21}$, home visits by a nurse practitioner ${ }^{20}$ or several disciplines ${ }^{20,22,25}$ and long-term outpatient comprehensive care by a geriatric clinic ${ }^{23,24,26}$. Nursing interventions targeted, for example, medications, symptom management, diet, activity, sleep, medical follow-up and emotional status. ${ }^{20}$ The interdisciplinary team in the studies by Burns and colleagues $(1995,2000)$ focused on functional limitations, gait impairment, incontinence, polypharmacy, depression, cognitive impairments and caregiver needs. ${ }^{23,24}$ Out of the seven transmural care interventions, one study reports significant effects in favour of the experimental group. ${ }^{19}$

Pointing out effective elements is difficult. In the study by McCusker and colleagues (2001), a two-step screening approach including the ISAR screening too was used.$^{19}$ This screening tool, specifically designed and evaluated for identifying seniors at high risk, might have increased the efficacy of the selection of participants. In the study by McCusker and colleagues (2001), treatment was individualised and clients were seen by a variety of disciplines. ${ }^{19}$ Referrals were made to the physician, local community, health centre, geriatric outpatient clinic and other community services. Comparable features, however, are present in the other CGA 'transmural care' studies that did not report effectiveness on disability. 
Community-based care: assessment followed by referrals and recommendations $(n=4)$

Eligible participants for these four studies were identified by a self-administered posta questionnaire ${ }^{27,28}$, telephone interview ${ }^{29}$ or an interviewer-assisted self-administered screening ${ }^{30}$. Extensive assessment was performed by a nurse during home visits ${ }^{27,28}$ or by a team in community-based clinics ${ }^{29,30}$. Medication, mood, cognition, vision hearing, blood pressure, gait and balance, orthostatic hypotension, environmental risk of fall, malnutrition, incontinence and social support were part of the assessment in the studies by Hebert and colleagues (2001) and Robichaud and colleagues (2000). 27,28 In all studies, the assessment was followed by recommendations to the general practitioner. In the study by Hebert and colleagues (2001) and Robichaud and colleagues (2000) recommendations to other health professionals were also made and bi-monthly or monthly phone follow-up interviews took place. ${ }^{27,28}$ In the study by Silverman and colleagues (1995), the treatment plan was discussed with the patient and family. ${ }^{29}$ Preparation of the patient and caregiver for the meeting with the general practitioner was part of the intervention in the study by Reuben and colleagues (1999). ${ }^{30}$

Out of these four community-based CGA studies, only the study by Reuben and colleagues (1990) found significant differences in disability in favour of the experimenta group..$^{30}$ The near-significant effect that Robichaud (2000) and colleagues found did not reach significance in the study by Hebert and colleagues (2001) in the evaluation of the same intervention among a larger group of participants. ${ }^{27,28}$ As all other three interventions have similar features to those of Reuben and colleagues ${ }^{30}$, elements that produce effectiveness in this type of CGA cannot be identified.

Community-based care: assessment followed by treatment $(n=15)$

Two out of fifteen studies are about the same intervention. ${ }^{31,32}$ All interventions were delivered by an interdisciplinary team consisting of at least two disciplines. Members of the interdisciplinary team were, for example, general practitioners, geriatricians, nurses, nurse practitioners, social workers, allied health professionals or medica specialists. The core of the intervention was mainly delivered by primary care professionals. Professionals working in institutions, for example, a geriatric clinic, were, however, often involved in the assessment and the development of the treatment plan, including referrals and recommendations. In addition, they could be consulted about the delivery of supplementary treatment if needed. In many interventions one person from the interdisciplinary team, often a nurse (practitioner), had the role of a case-manager. ${ }^{33-39}$ The case manager was responsible for planning, coordination, monitoring, and evaluation of assessment and treatment. The assessment in the 15 studies focused on medical conditions and general status of the participants (functional, psychosocial, cognitive, affective and nutritional), personal resources and preferences, caregiver's capabilities and other environmental factors. The focus of the interventions varied strongly across studies. Issues covered were falls, balance, urinary incontinence, functional impairment, depression, cognitive deficits, nutrition mobility, medication, social support, service use, communication, environmental aspects and financial needs.

Nearly all interventions were delivered in an individual format, except for Phelan and colleagues (2004), who combined individual sessions with group sessions (exercise and self-management classes). ${ }^{38} \mathrm{Home}$ visits ${ }^{34,37,39-41}$, telephone calls ${ }^{39}$ or a combination of both $35,36,38,39,42$ are repeatedly used to address frail older people. In three studies assessment and treatment were done in a (geriatric) clinic..$^{31,32,43}$ Two studies combined clinic visits, home visits and telephone contacts. ${ }^{39,44}$

Out of fifteen CGA studies, nine report significant differences in disability in favour of the experimental group. . $3,36-38,40,42-45$ There are some features that promising interventions have in common. Out of the nine studies, seven studies report the use of an individualised treatment plan that is based on a multidimensiona assessment. ${ }^{34,36,37,40,43,44,46} \mathrm{~A}$ case manager had a key role during the intervention process in six out of nine effective studies. ${ }^{34-38,40}$ Regular team meetings were applied in four studies. $36,37,40,43$ In seven out of the nine studies, on-going assessment, evaluation and monitoring are described as a feature of the intervention. ${ }^{34,35,37,38,40,44,4}$ Therefore a combination of home visits and telephone contacts is often used. $35,36,38,39,42$ Out of nine studies, four interventions also intervene in factors in the social and physical environment of frail older people. ${ }^{35,37,44,46}$ Health education is applied in four interventions. ${ }^{35,37,39,44} \mathrm{~A}$ complication in drawing conclusions about elements that contributed to effectiveness is the presence of identical features in the six clinica trials that did not show any differences for disability. There is, however, some indication that case management, individualised treatment, multidimensiona assessment and on-going evaluation and monitoring are relevant features in this type of CGA interventions. A combination of home visits and telephone contacts and regular team meetings seems to be promising. Health education may also be an important component for future interventions.

Physical Exercise programmes $(n=12)$

Twelve studies describing physical exercise interventions were found. Physical exercise interventions for community-dwelling frail older people show a large variation in content, duration, intensity, balance between supervised and non-supervised sessions and level of individualisation. Five interventions were single-component, focusing mainly on lower extremity strength. ${ }^{47-51}$ The other seven were multi-component, addressing a variety of physical parameters such as endurance, flexibility balance and strength. ${ }^{52-58}$ All interventions were offered by professionals in physica exercise, mostly physical therapists. In most studies, participants performed at leas three exercise sessions a week. ${ }^{47-50,52,54-58}$ Strength training was usually comprised of 
a number of exercises for improvement of lower body strength using weights ${ }^{59}$, elastic bands ${ }^{47-49}$ or training machines ${ }^{51}$. Among the multi-component exercise programmes, three addressed several parameters in all exercise sessions. ${ }^{56-58}$ These also belong to the shortest multicomponent exercise programmes (10-12 weeks). The longest multi-component programmes lasted 9 months ${ }^{52}, 12$ months $^{54}$ and 18 months $^{55}$. In two studies, different phases (respectively 3 or 6 months) were distinguished with a focus on specific physical parameters in each phase. ${ }^{52,55}$ The content of the programme of Gill and colleagues ${ }^{54}$ was more individualised, as the outcomes of an extensive assessment directed the programme. Among the physical exercise programmes, there is a large difference between the number of supervised and non-supervised sessions. For example, Binder and colleagues (2002) report a total of 108 supervised sessions, while Jette and colleagues (1999) report only two home visits by a physical therapist. The participants exercised non-supervised, supported by techniques to enhance adherence. ${ }^{49,52}$

Out of twelve physical exercise programmes, four report significant positive effects for disability. Of the single-component physical exercise programmes $(n=5)$, one reports positive effects. Jette and colleagues (1999) found evidence for the effect of resistance training using elastic bands. ${ }^{49}$ For three (out of seven) multi-component programmes, significant positive effects are reported on the disability outcome. ${ }^{52,54,58}$ In these three studies, participants were included according to physical frailty indicators and programmes were relatively intensive with at least three exercise sessions per week. Binder and colleagues (2002) included only moderate physically frail older people..52 Gill and colleagues (2002) found that only moderate physically frail older people benefited from their intervention. ${ }^{54}$

Nutrition $(n=3)$

Studies that investigated the effect of single nutritional interventions focused on macronutrient status (nutrient-dense protein energy liquid) ${ }^{60}$, micronutrient status (fruits and dairy products enriched with vitamins and minerals) ${ }^{53}$ or both (meals and snacks providing $100 \%$ of macro- and micronutrient requirements) ${ }^{61}$. In the study by Kretser and colleagues (2003) participants received 21 meals and 14 snacks every week for 6 months, accounting for most of the daily nutritional intake. ${ }^{61}$ In Chin A Paw and colleagues' study (2001), participants were asked to eat the products in addition to their daily diet or as a replacement (for 17 weeks). ${ }^{53}$ In Payette and colleagues study (2002), the liquid product was an addition to the usual daily dietary intake for 16 weeks. ${ }^{60}$ Additional support included monthly home visits by dieticians and a phone call every 2 weeks with nutritional counselling and encouragement ${ }^{60}$ or daily additional phone calls from older adult volunteers to provide a measure of safety and socialisation ${ }^{61}$
Despite an observed effect on total energy intake ${ }^{60}$ and weight gain ${ }^{60,61}$, none of the three nutritional intervention studies report evidence for the effect of nutritional interventions on disability. ${ }^{53,60,61}$

Technological interventions $(n=2)$

Environmental adaptations are often part of the multifactorial and multidisciplinary programmes described under CGA. Two reports were found on the effect of single assistive devices and home modifications ${ }^{62}$ and smart technology on disability ${ }^{63}$. In the study by Mann and colleagues (1999), an occupational therapist performed a comprehensive functional assessment of the person and the home followed by recommendations for assistive devices and/ or home modifications. ${ }^{62}$ Participants were trained in the use of the devices and follow-up continued with assessment and provision of assistive technology as needs changed. Over about 18 months compute and internet facilities were provided to the participants in the study by Tomita and colleagues (2007). ${ }^{63} \mathrm{~A}$ computer engineer adapted the computer to ensure a good fit with its users. Furthermore, smart technology, like door and window sensors, motion sensors and remote control for lamps and radio, was installed. The level of automatisation was determined by the participants' desire and the capacity of the house. Participants received instruction from a geriatric nurse who was a specialist in computer instruction.

Both studies report significant differences on disability in favour of the experimenta group. ${ }^{62,63}$ In both interventions there is an emphasis on the adaptation of technology towards the needs of the clients and on intensive instruction in the use of devices.

Other interventions $(n=6)$

In this group, six interventions are described. Latham and colleagues (2003) studied the effect of Vitamin D. Participants in the experimental group received $1.25 \mathrm{mg}$ calciferol..$^{50}$ Giannini and colleagues (2007) focused on the effect of home care attendance (between 4 and 24 hours) by professionals with training in the care of frail older people. ${ }^{64}$ In two studies ${ }^{65,66}$ the intervention consisted of monthly group meetings (over 12 months) with a physician and a nurse.65,66 The meetings focused on health promotion and control. One intervention contains three inpatient rehab periods with individual and group sessions plus home activation days and home visits for persona hygiene and assistive devices ${ }^{67}$ Liang and colleagues (1986) used a stepped-up treatment (on top of nursing and social services) provided by a physician and physical therapis focusing on assistive devices and home modification and supervised exercises. ${ }^{68}$

Out of the six interventions, Giannini and colleagues (2007) report significant positive effects on disability in favour of the experimental group. ${ }^{64}$ They describe how the home care attendance, provided by a nurse trained in the care of frail older people, was delivered according to a programme established by a Geriatric Evaluation Unit. Specific features of this programme are lacking. 


\section{Discussion}

This review offers a comprehensive overview of the content of interventions targeted at disability prevention in community-dwelling frail older people. In total, 48 clinical trials evaluating 49 interventions aimed at disability prevention were identified. The majority of trials in 46 RCTs and two CCTs were conducted in the field of comprehensive geriatric assessment (CGA) $(n=26)$. Studies of physical exercise programmes are the second largest group of interventions $(n=12)$. There is a small number of studies that specifically focus on the effect of technology $(n=2)$ or nutrition $(n=3)$ on disability in community-living frail older people. Both environmental adaptations and nutrition, however, are frequently mentioned as part of the CGA studies. The results show large diversity within and between the groups of interventions in terms of content disciplines involved, duration, intensity and setting.

The reported effectiveness of interventions is not consistent. Eighteen of the 49 interventions reported significant positive effects for disability for the experimental group. As most studies did not include long-term follow-up measurement, the preventive potential of interventions remains unclear for the time period after the completion of the intervention. The most promising findings were found for technology (but only two studies), CGA 'assessment followed by treatment' (nine out of fifteen studies) and, to a lesser extent, physical exercise programmes (four out of twelve). Technology, adapted to the needs of the frail older people and well-taught, may be very effective in preventing disability in community-dwelling frail older people, though more research is needed in this area. Most trials were conducted in CGA, but the reported effectiveness of CGA for community-dwelling frail older people is inconsistent. Comparable findings are reported in previous reviews on CGA.16,69,70 Individualised, multifactorial and multidisciplinary assessments and interventions, case management and on-going monitoring (long-term management) seem to be essential elements for effective CGA. The mixed results of physical exercise programmes hamper the identification of effective elements. There is, however, some indication that multi-component high intensive physical exercise programmes may be promising, especially for moderate physically frail community-dwelling older people.

This overview has some limitations. The use of the term frailty in the literature is relatively new. Although reference lists were checked, a limitation of this overview is that older studies might not have been identified as we searched with terms for frailty and its synonyms. Another risk in terms of publication bias is the selection of 4602 studies on title-level that may have resulted in the exclusion of relevant articles. Only randomised controlled trials were selected as we considered these as a (minimal) standard for quality. Some caution in interpretation of results is warranted as the methodological quality of the studies was not assessed. Furthermore, we analysed the results in a qualitative way. As a consequence effect sizes of interventions were not calculated or taken into account. And last, owing to small sample sizes, trials may have been underpowered to detect differences in the self-reported measures for disability.

Recent literature on disability development suggests that disability is multifactoria in nature. Stuck and colleagues (1999) reported that risk factors for developing disability in community-dwelling older people are cognitive impairment, depression, comorbidity, increased and decreased body mass index, lower extremity functiona limitation, low frequency of social contacts, and low level of physical activity. ${ }^{71}$ Femia and colleagues (2003) suggest that although disease conditions and physica impairments are as risk factors strongly related to an individual's ability to carry out activities of daily living, other factors like the beliefs about one's health (e.g., subjective health), motivation and self-efficacy are potentially as important as the ability to perform them. Therefore, it seems that a combination of risk factors and other factors plays a role in the development of disability implying that disability prevention in frail older people is complex. In view of this complexity, the Dutch National Health Counc recently pointed out the need for research on 'function-oriented prevention'. The Health Council stated that knowledge of the effectiveness of preventive interventions on disability is fragmented, heterogenic, and still lacking in a variety of areas. ${ }^{72}$ How might the findings of this overview influence future interventions for community-dwelling frail older people?

In the light of this overview, future interventions may be directed toward tailor-made, multidisciplinary and multifactorial interventions, with individualised assessment and interventions conducted by a (primary) care team, involving case management, and long-term follow-up. These tailor-made programmes may include a physical exercise component for moderate physically frail older people and technology component tailored to the needs of older people. Several new elements might be added to future interventions. For example, technology for monitoring health conditions and enhancing compliance and communication between health professionals and clients is available and can be incorporated in interventions for frail older people..$^{73}$ Techniques for enhancing self-management abilities in older people have been described in several studies ${ }^{74,75}$ and seem applicable to, and promising for, community-dwelling frail older people. ${ }^{74,75} \mathrm{~A}$ systematic approach toward enabling community-dwelling frail older people to be engaged in meaningful social and productive activities might also be effective to prevent disability, as it fosters natura motivation and self-efficacy in older people. Recent studies show the potential of meaningful activities as a core of preventive programmes. ${ }^{76,77}$ It is likely that the development of interventions for community-living frail older people has still some way to go. Further research with a focus on interventions that can prevent or delay disability in community-dwelling frail older people is necessary. 


\section{References}

1. Aminzadeh F, Dalziel WB, Molnar FJ. Targeting frail older adults for outpatient comprehensive geriatric assessment and management services: An overview of concepts and criteria. Rev Clin Gerontol. 2002:12(1):82-92

2. Espinoza S, Walston JD. Frailty in older adults: insights and interventions. Cleve Clin J Med 2005;72(12): :1105-12.

3. Storey ET, R.L. Understanding and ameliorating frailty in the elderly. Top Geriatr Rehabil. 2004;20(1):4-13. Hooi WC BH. A review on models and perspectives on frailty in older persons. SGH Proceedings 2005. 2005.

5. Markle-Reid M, Browne G. Conceptualizations of frailty in relation to older adults. J Adv Nurs. 2003;44(1):58-68

6. Slaets JPJ. Vulnerability in the elderly: frailty. Med Clin North Am. 2006;90(4):593-601.

7. Hogan DB, MacKnight C, Bergman H. Models, definitions, and criteria of frailty. Aging Clin Exp Res. 2003;15(3 Suppl):1-29.

8. Lally F, Crome P. Understanding frailty. Postgrad Med J. 2007;83(975):16-20.

9. Aminzadeh F, Byszewski A, Dalziel WB, Wilson M, Deane N, Papahariss-Wright S. Effectiveness of outpatient geriatric assessment programs: exploring caregiver needs, goals, and outcomes. J Geronto Nurs. 2005;31(12):19-25.

10. Levers MJ, Estabrooks CA, Ross Kerr JC. Factors contributing to frailty: literature review. J Adv Nurs 2006;56(3):282-91

1. Jette AM. Toward a common language for function, disability, and health. Phys Ther. 2006;86(5):726-34.

12. Cutler DM. Declining disability among the elderly. Health Aff (Millwood). 2001;20(6):11-27.

Ferrucci L, Guralnik JM, Studenski S, Fried LP, Cutler GB, Jr., Walston JD. Designing randomized, controlled trials aimed at preventing or delaying functional decline and disability in frail, older persons: (3)

4. Bours GJJW, Ketelaars C.A.J., Frederiks CMA, Huyer Abu-Saad H, Wouters EFM. The effects of aftercare on chronic patients and frail elderly patients when discharged from hospital: a systematic review. J Ad Nurs. 1998:27(5):1076-86

15. Mason A, Weatherly H, Spilsbury K, Arksey H, Golder S, Adamson J, Drummond M, Glendinning C. A systematic review of the effectiveness and cost-effectiveness of different models of community-based respite care for frail older people and their carers. Health Technol Assess. 2007;11(15):1-157, iii.

6. Wieland $\mathrm{D}$. The effectiveness and costs of comprehensive geriatric evaluation and management. Crit Rev Oncol Hematol. 2003;48(2):227-37.

17. Avlund K. Disability in old age. Longitudinal population-based studies of the disablement process. Dan Med Bull. 2004;51(4):315-49.

18. Rubenstein LZ, Siu AL, Wieland D. Comprehensive geriatric assessment: toward understanding its efficacy. Aging (Milano). 1989;1(2):87-98

19. McCusker J, Verdon J, Tousignant P, de Courval LP, Dendukuri N, Belzile E. Rapid emergency department intervention for older people reduces risk of functional decline: results of a multicenter randomized tria. J Am Geriatr Soc. 2001;49(10):1272-81.

20. Naylor MD, Brooten D, Campbell R, Jacobsen BS, Mezey MD, Pauly MV, Schwartz JS. Comprehensive discharge planning and home follow-up of hospitalized elders: a randomized clinical trial. JAMA. 1999;281(7):613-20.

21. Siu AL, Kravitz RL, Keeler E, Hemmerling K, Kington R, Davis JW, Mitchell A, Burton TM, Morgenstern H Beers MH, Reuben DB. Postdischarge geriatric assessment of hospitalized frail elderly patients. Arch Intern Med. 1996;156(1):76-81.

22. Hughes SL, Cummings J, Weaver F, Manheim LM, Conrad KJ, Nash K. A randomized trial of Veterans Administration home care for severely disabled veterans. Med Care. 1990;28(2):135-45

23. Burns R, Nichols LO, Graney MJ, Cloar FT. Impact of continued geriatric outpatient management on health outcomes of older veterans. Arch Intern Med. 1995;155(12):1313-8.
24. Burns R, Nichols LO, Martindale-Adams J, Graney MJ. Interdisciplinary geriatric primary care evaluation and management: two-year outcomes. J Am Geriatr Soc. 2000;48(1):8-13.

25. Oktay JS, Volland PJ. Post-hospital support program for the frail elderly and their caregivers: a quasiexperimental evaluation. Am J Public Health. 1990;80(1):39-46.

26. Rubin CD, Sizemore MT, Loftis PA, de Mola NL. A randomized, controlled trial of outpatient geriatric evaluation and management in a large public hospital. J Am Geriatr Soc. 1993;41(10):1023-8.

27. Hébert R, Robichaud L, Roy PM. Bravo G. Voyer L. Efficacy of a nurse-led multidimensional preventive

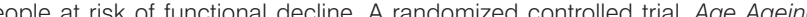
2001;30(2): 147-53.

28. Robichaud L, Hebert R, Roy PM, Roy C. A preventive program for community-dwelling elderly at risk of functional decline: a pilot study. Arch Gerontol Geriatr. 2000;30(1):73-84

29. Silverman M, Musa D, Martin DC, Lave JR, Adams J, Ricci EM. Evaluation of outpatient geriatric assessment: a randomized multi-site trial. J Am Geriatr Soc. 1995;43(7):733-40.

30. Reuben DB, Frank JC, Hirsch SH, McGuigan KA, Maly RC. A randomized clinical trial of outpatien comprehensive geriatric assessment coupled with an intervention to increase adherence to recommendations. J Am Geriatr Soc. 1999;47(3):269-76.

31. Engelhardt JB, Toseland RW, O'Donnell JC, Richie JT, Jue D, Banks S. The effectiveness and efficiency of outpatient geriatric evaluation and management. J Am Geriatr Soc. 1996;44(7):847-56.

32. Toseland RW, O'Donnell JC, Engelhardt JB, Hendler SA, Richie JT, Jue D. Outpatient geriatric evaluation and management. Results of a randomized trial. Med Care. 1996;34(6):624-40.

33. Gagnon AJ, Schein C, McVey L, Bergman H. Randomized controlled trial of nurse case management of frail older people. J Am Geriatr Soc. 1999;47(9):1118-24.

34. Landi F, Onder G, Tua E, Carrara B, Zuccala G. Gambassi G, Carbonin P. Bernabei R. Impact of a new assessment system, the MDS-HC, on function and hospitalization of homebound older people: controlled clinical trial. J Am Geriatr Soc. 2001:49(10): 1288-93.

35. Markle-Reid M, Browne G, Weir R, Gafni A, Roberts J, Henderson SR. The effectiveness and efficiency of home-based nursing health promotion for older people: review of the literature. Med Care Res Rev 2006:63(5):531-69.

36. Melin AL, Bygren LO. Efficacy of the rehabilitation of elderly primary health care patients after short-stay hospital treatment. Med Care. 1992;30(11):1004-15.

37. Melis RJ, van Eijken MI, Teerenstra S, van Achterberg T, Parker SG, Borm GF, van de Lisdonk EH, Wensing M, Rikkert MG. A randomized study of a multidisciplinary program to intervene on geriatric syndromes in vulnerable older people who live at home (Dutch EASYcare Study). J Gerontol A Biol Sci Med Sci. 2008;63(3):283-90.

38. Phelan EA, Williams B, Penninx BW, LoGerfo JP, Leveille SG. Activities of daily living function and disability in older adults in a randomized trial of the health enhancement program. J Gerontol A Biol Sci Med Sci. 2004;59(8):838-43

39. Rubenstein LZ, Alessi CA, Josephson KR, Trinidad Hoyl M, Harker JO, Pietruszka FM. A randomized tria of a screening, case finding, and referral system for older veterans in primary care. J Am Geriatr Soc. 2007;55(2):166-74.

40. Bernabei R, Landi F, Gambassi G, Sgadari A, Zuccala G, Mor V, Rubenstein LZ, Carbonin P. Randomised trial of impact of model of integrated care and case management for older people living in the community. BMJ. 1998;316(7141):1348-51.

41. Rockwood K, Stadnyk K, Carver D, MacPherson KM, Beanlands HE, Powell C, Stolee P, Thomas VS, Tonks RS. A clinimetric evaluation of specialized geriatric care for rural dwelling, frail older people. J Am Geriatr Soc. 2000;48(9):1080-5.

42. Gitlin LN, Winter L, Dennis MP, Corcoran M, Schinfeld S, Hauck WW. A randomized trial of a multicomponent home intervention to reduce functional difficulties in older adults. J Am Geriatr Soc 2006;54(5):809-16.

43. Cohen HJ, Feussner JR, Weinberger M, Carnes M, Hamdy RC, Hsieh F, Phibbs C, Courtney D, Lyles KW, May C, McMurtry C, Pennypacker L, Smith DM, Ainslie N, Hornick T, Brodkin K, Lavori P. A controlled tria of inpatient and outpatient geriatric evaluation and management. N Engl J Med. 2002;346(12):905-12. 
44. Boult C, Boult LB, Morishita L, Dowd B, Kane RL, Urdangarin CF. A randomized clinical trial of outpatient geriatric evaluation and management. J Am Geriatr Soc. 2001;49(4):351-9.

45. Markle-Reid M, Weir R, Browne G, Roberts J, Gafni A, Henderson S. Health promotion for frail older home care clients. J Adv Nurs. 2006;54(3):381-95.

46. Gittin LN, Hauck WW, Winter L, Dennis MP, Schulz R. Effect of an in-home occupational and physical therapy intervention on reducing $m$

47. Boshuizen HC, Stemmerik L, Westhoff MH, Hopman-Rock M. The effects of physical therapists' quidance on improvement in a strength-training program for the frail elderly. J Aging Phys Act. 2005:13(1):5-22

48. Chandler JM. Duncan PW Kochersberger G, Studenski S Is lower extremity strength gain associated with improve Dent in physical performance and disability in frail community-dwelling glders? Arch Phys Med Rehabil. 1998;79(1):24-30.

- it's never too late: the strong-for-life program. Am J Public Health. 1999;89(1):66-72.
.

50. Latham NK Anderson CS, Lee A, Bennett DA, Moseley A, Cameron ID. A randomized, controlled trial of quadriceps resistance exercise and vitamin $\mathrm{D}$ in frail older people: the Frailty Interventions Trial in Elderly Subjects (FITNESS). J Am Geriatr Soc. 2003;51(3):291-9.

51. Ota A, Yasuda N, Horikawa S, Fujimura T, Ohara H. Differential effects of power rehabilitation on physical performance and higher-level functional capacity among community-dwelling older adults with a slight degree of frailty. J Epidemiol. 2007;17(2):61-7.

52. Binder EF, Schechtman KB, Ehsani AA, Steger-May K, Brown M, Sinacore DR, Yarasheski KE, Holloszy JO. Effects of exercise training on frailty in community-dwelling older adults: results of a randomized controlled trial. J Am Geriatr Soc. 2002;50(12):1921-8.

53. Chin A Paw MJ, de Jong N, Schouten EG, Hiddink GJ, Kok FJ. Physical exercise and/ or enriched foods for functional improvement in frail, independently living elderly: a randomized controlled trial. Arch Phys Med Rehabil. 2001:82(6):811-7.

54. Gill TM, Baker DI, Gottschalk M, Peduzzi PN, Allore H, Byers A. A program to prevent functional decline in physically frail, elderly persons who live at home. $N$ Engl $J$ Med. 2002;347(14):1068-74.

55. King MB, Whipple RH, Gruman CA, Judge JO, Schmidt JA, Wolfson LI. The Performance Enhancemen Project: improving physical performance in older persons. Arch Phys Med Rehabil. 2002;83(8):1060-9.

56. Luukinen H, Lehtola S, Jokelainen J, Vaananen-Sainio R, Lotvonen S, Koistinen P. Prevention of disability by exercise among the elderly: a population-based, randomized, controlled trial. Scand J Prim Health Care. 2006;24(4):199-205

57. Timonen L, Rantanen T, Makinen E, Timonen TE, Tormakangas T, Sulkava R. Effects of a group-based exercise program on functional abilities in frail older women after hospital discharge. Aging Clin Exp Res. 2006; $18(1): 50-6$.

58. Worm CH, Vad E, Puggaard L, Stovring H, Lauritsen J, Kragstrup J. Effects of a multicomponent exercise program on functional ability in community-dwelling frail older adults. J Aging Phys Act. 2001;9(414-424).

59. Latham NK, Bennett DA, Stretton CM, Anderson CS. Systematic review of progressive resistance strength training in older adults. J Gerontol A Biol Sci Med Sci. 2004;59(1):48-61.

60. Payette H, Boutier V, Coulombe C, Gray-Donald K. Benefits of nutritional supplementation in free-living frail, undernourished elderly people: a prospective randomized community trial. J Am Diet Assoc. 2002;102(8):1088-95

61. Kretser AJ, Voss T, Kerr WW, Cavadini C, Friedmann J. Effects of two models of nutritional intervention on homebound older adults at nutritional risk. J Am Diet Assoc. 2003;103(3):329-36.

62. Mann WC, Ottenbacher KJ, Fraas L, Tomita M, Granger CV. Effectiveness of assistive technology and environmental interventions in maintaining independence and reducing home care costs for the fral elderly. A randomized controlled trial. Arch Fam Med. 1999;8(3):210-7.

63. Tomita MR, Mann WC, Stanton K, Tomita AD, Sundar V. Use of currently avallable smart home technology for frail elders; process and outcomes. Top Geriatr Rehabil 2007;23(1):24-34

64. Giannini R, Petazzoni E, Savorani G, Galletti L, Piscaglia F, Licastro F, Bolondi L, Cucinotta D. Outcomes from a program of home care attendance in very frail elderly subjects. Arch Gerontol Geriatr. 2007;44(2):95-103
65. Beck A, Scott J, Williams P, Robertson B, Jackson D, Gade G, Cowan P. A randomized trial of group outpatient visits for chronically ill older HMO members' the Cooperative Health Care Clinic. J Am Geriat Soc. 1997; $45(5): 543-9$

66. Scott JC, Conner DA, Venohr I, Gade G, McKenzie M, Kramer AM, Bryant L, Beck A. Effectiveness of a group outpatient visit model for chronically ill older health maintenance organization members: a 2-year randomized trial of the cooperative health care clinic. J Am Geriatr Soc. 2004;52(9):1463-70.

67. Ollonqvist K, Aaltonen T, Karppi SL, Hinkka K, Pontinen S. Network-based rehabilitation increases formal support of frail elderly home-dwelling persons in Finland. randomised controlled trial. Health Soc Care Community. 2008;16(2):115-25.

68. Liang $\mathrm{MH}$, Partridge AJ, Gall V, Taylor J. Evaluation of a rehabilitation component of home care for homebound elderly. Am J Prev Med. 1986;2(1):30-4.

69. Beswick AD, Rees K, Dieppe P. Ayis S, Gooberman-Hill R. Horwood J, Ebrahim S. Complex intervention to improve physical function and maintain independent living in elderly people: a systematic review and meta-analysis. Lancet. 2008;371(9614):725-35.

70. Wieland D. Hirth V Comprehensive geriatric assessment. Cancer Control. 2003:10(6):454-62

71. Stuck AE, Walthert JM, Nikolaus T, Bula CJ, Hohmann C, Beck JC. Risk factors for functional status decline in community-living elderly people: a systematic literature review. Soc Sci Med. 1999;48(4):445-69.

72. RVZ. Preventie bij ouderen: focus op zelfredzaamheid. Den Haag: Gezondheidsraad, 2009/72009.

73. Botsis $T$, Hartvigsen G. Current status and future perspectives in telecare for elderly people suffering from chronic diseases. J Telemed Telecare. 2008;14(4):195-203

74. Frieswijk N, Steverink N, Buunk BP, Slaets JPJ. The effectiveness of a bibliotherapy in increasing the selfmanagement ability of slightly to moderately frail older people. Patient Educ Couns. 2006;61(2):219-27.

75. Lamers F, Jonkers CC, Bosma H, Diederiks JP, van Eijk JT. Effectiveness and cost-effectiveness of a minimal psychological intervention to reduce non-severe depression in chronically ill elderly patients: the mised controlled trial [ISRCTN92331982]. BMC Public Health. 2006;6:161-70.

76. Fried LP, Carlson MC, Freedman M, Frick KD, Glass TA, Hill J, McGill S, Rebok GW, Seeman T, Tielsch J, Wasik BA, Zeger S. A social model for health promotion for an aging population: initial evidence on the Experience Corps model. J Urban Health. 2004;81(1):64-78.

77. Graff MJ, Vernooij-Dassen MJ, Thijssen M, Dekker J, Hoefnagels WH, Olde Rikkert MG. Community based occupational therapy for patients with dementia and their care givers: randomised controlled tria. BMJ. 2006;333(7580):1196 


\section{4}

The reduction of disability

in community-dwelling frail older people:

design of a two-arm cluster randomised

controlled trial

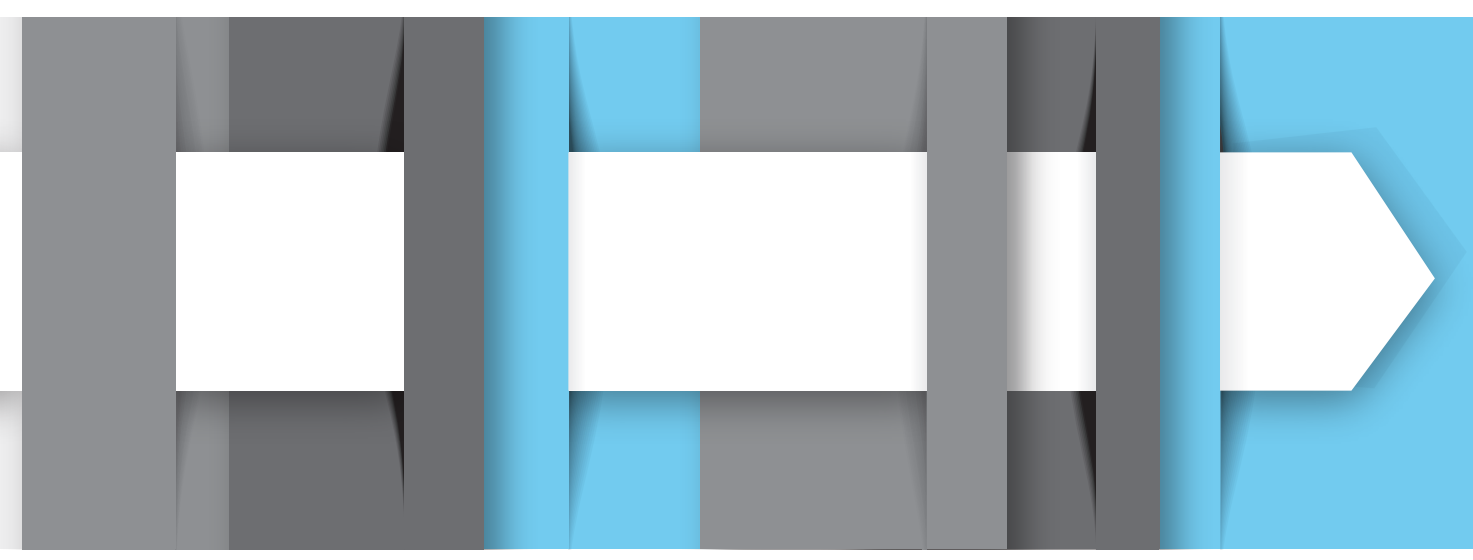

This chapter was published as:

Metzelthin, SF, van Rossum, E, de Witte, LP, Hendriks, MRC, Kempen, GIJM BMC Public Health 2010;10:511 


\section{Abstract}

Background: Frailty among older people is related to an increased risk of adverse health outcomes such as acute and chronic diseases, disability and mortality Although many intervention studies for frail older people have been reported, only a few have shown positive effects regarding disability prevention. This article presents the design of a two-arm cluster randomised controlled trial on the effectiveness, costeffectiveness and feasibility of a primary care intervention that combines the most promising elements of disability prevention in community-dwelling frail older people. Methods/ design: In this study twelve general practitioner practices were randomly allocated to the intervention group (6 practices) or to the control group (6 practices). Three thousand four hundred ninety-eight screening questionnaires including the Groningen Frailty Indicator (GFI) were sent out to identify frail older people. Based on their GFI score (25), 360 participants will be included in the study. The intervention group will receive an interdisciplinary primary care intervention. After a comprehensive assessment by a practice nurse and additional assessments by other professionals, if needed, an individual action plan will be defined. The action plan is related to a flexible toolbox of interventions, which will be conducted by an interdisciplinary team. Effects of the intervention, both for frail older people and their informal caregivers, wil be measured after 6,12 and 24 months using postal questionnaires and telephone interviews. Data for the process evaluation and economic evaluation will be gathered continuously over a 24-month period.

Discussion: The proposed study will provide information about the usefulness of an interdisciplinary primary care intervention. The postal screening procedure was conducted in two cycles between December 2009 and April 2010 and turned out to be a feasible method. The response rate was $80 \%$. According to GFI scores $29 \%$ of the respondents can be considered as frail (GFI $\geq 5)$. Nearly half of them $(48 \%)$ were willing to participate. The baseline measurements started in January 2010. In February 2010 the first older people were approached by the practice nurse for a comprehensive assessment. Data on the effect, process, and economic evaluation will be available in 2012

Trial Registration: ISRCTN31954692

\section{Background}

Frailty is highly prevalent in older people; up to $40 \%$ of this population is estimated to be frail and an increasing trend is expected. ${ }^{1}$ Frailty is related to an increased risk of adverse health outcomes such as acute and chronic diseases, disability and mortality. ${ }^{2-4}$ Disability is defined as difficulty or dependency in the execution of the activities of daily living and is associated with increased healthcare utilisation and related costs. ${ }^{5}$ Next to professional healthcare services, informal caregivers are a source of long-term care for frail older people. However, demographic and socia changes such as fewer children, high divorce rates and other caregiving responsibilities reduce the ability of informal caregivers to provide this care. ${ }^{6}$ In view of a growing frail older population and restraints in healthcare expenditure and availability of informa care, disability in frail older people is a public health problem and its prevention is considered to be a priority for research and clinical practice in geriatric care.,3

During the last few decades various interventions have been developed targeting frail older people. These show a large diversity in terms of content, disciplines involved, duration, intensity and setting. Most studies have been conducted in the field of comprehensive geriatric assessment (CGA) or physical exercise. Comprehensive geriatric assessment has been defined as 'a multidimensional, often interdisciplinary, diagnostic process intended to determine a frail older person's medical, psychosocial, and functional capabilities and problems, with the objective of developing an overall plan for treatment and long-term follow-up.. 9 Reviews have shown that the reported effectiveness of CGA studies is inconsistent., ${ }^{8,10-12}$ Physical exercise programs for frail older people are mostly effective on frailty components such as physical fitness and balance, but they are less effective on disability outcomes. ${ }^{13}$ The use of technology may be effective but more research is needed in this area..$^{14,15}$ In conclusion, only a small number of intervention studies have shown beneficial effects with regard to disability prevention and most studies did not report on the long-term effects. ${ }^{13}$

A narrative review by Daniëls and colleagues (2010) suggested that future community care interventions for frail older people should be directed towards tailor-made, multidisciplinary and multifactorial interventions, with individualised assessment and interventions conducted by a (primary) care team, involving case management and long-term follow-up. ${ }^{8}$ These programmes may include a physica exercise component for moderately physically frail older people and a technology component tailored to the needs of older people. ${ }^{8}$ Other promising elements are techniques for enhancing self-management abilities ${ }^{16-19}$ and engagement in meaningful social and productive activities, as these foster natural motivation and self-efficacy in older people 20,21 
The present study focuses on a primary care intervention that combines the most promising elements suggested above. This two-arm cluster randomised controlled trial aims to investigate (1) the effectiveness of the intervention with regard to disability (primary outcome) and several secondary functional outcomes, (2) the impact of the intervention on the central informal caregiver with respect to perceived burden on health-related quality of life and (3) the impact of the intervention on healthcare utilisation and related costs. In addition, (4) the feasibility of the intervention, including the adherence, will be studied. This article presents the study design and reports on the results of the screening procedure.

\section{Methods/ design}

Study Design

All general practitioner (GP) practices in the region of Sittard (the Netherlands) and its surroundings were invited to take part in the study, with the restriction that they had no current active and systematic policy for the detection and follow-up of frail older people. In total, 24 GP practices were interested, of which 12 were randomly selected for the study. Cluster randomisation was applied to allocate the selected practices to the intervention group (six practices) or the care as usual group (six practices). A flow diagram of the study design is shown in Figure 1.

Effects of the intervention, both for frail older people and their informal caregivers, will be measured after 6, 12 and 24 months using postal questionnaires and telephone interviews. Data on healthcare utilisation and related costs will be gathered continuously over a 24-month period from health insurance registries and registries of GPs and regional hospitals (economic evaluation). A process evaluation will be conducted by means of diaries, questionnaires and semi-structured interviews.

The study obtained approval by the Medical Ethical Committee of the Maastrich University/Academic Hospital Maastricht in the Netherlands in 2009 (MEC 09-3-067).

\section{Participants and recruitment}

The study focuses on community-dwelling frail older people ( $\geq 70$ years). Those who were terminally ill, were confined to bed, had severe cognitive or psychologica impairments or were unable to communicate in Dutch were excluded based on the advice of their GP. Consequently, frail older people were screened for frailty. On average, 300 screening questionnaires per practice were sent out depending on the number of older people registered (range 200-350). Where practices had more olde people who fulfilled the inclusion criteria, a random selection was drawn. On behal of their GP, the selected older people received the Groningen Frailty Indicator (GFI) to screen for frailty. ${ }^{22}$ In the literature, a score of four or higher (range $0-15$ ) is proposed

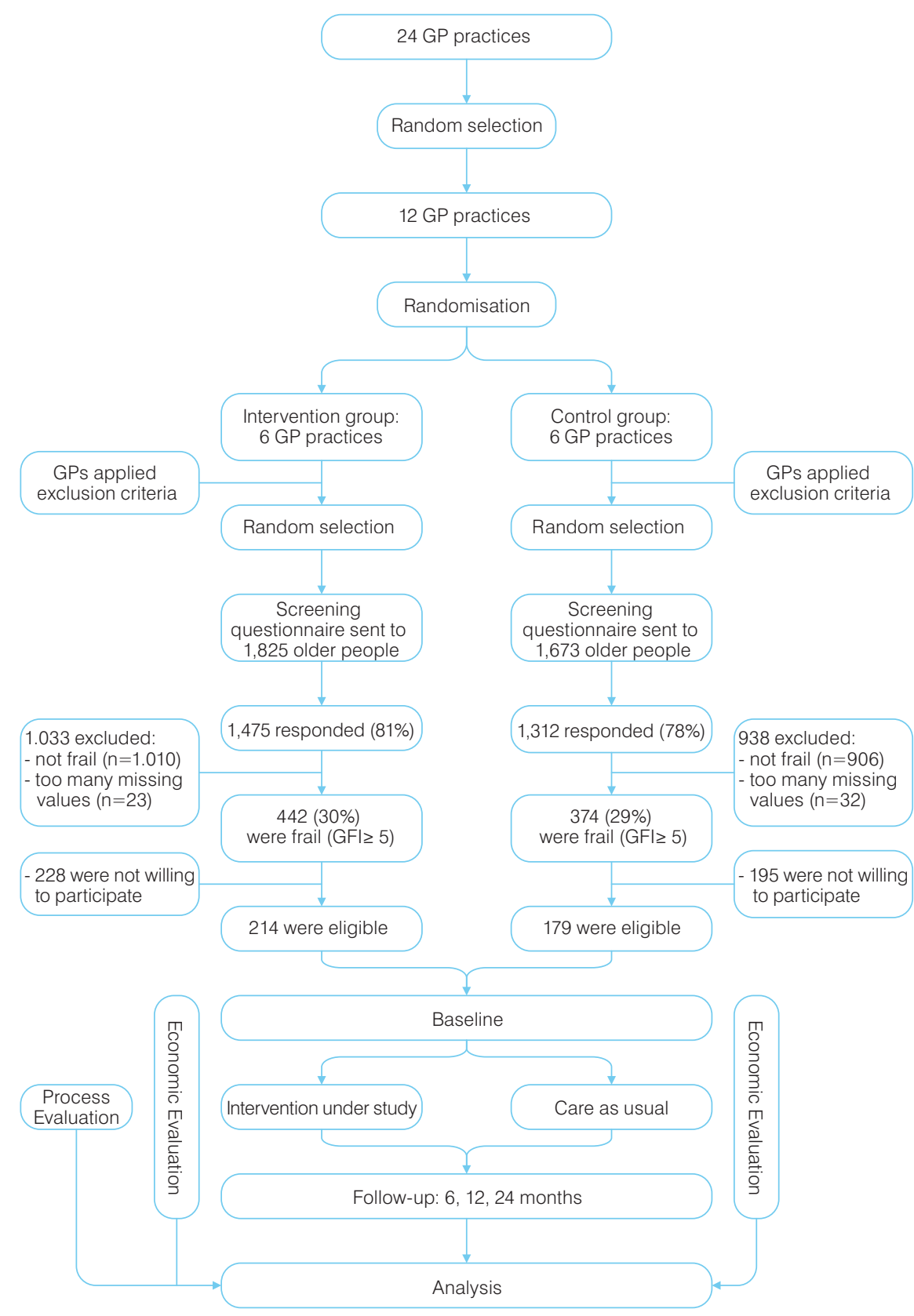

Figure 1 Study design. 
as cut-off point for moderately to severely frail older people. 22,23 However, this study focuses on people who are considerably frail. Therefore a cut-off score of five was chosen. A letter from the GP, an information leaflet, an informed consent form and a postage free return envelope were included with the screening questionnaire. In total, a sample size of 3,498 older people was addressed (see Figure 1). Reminders were sent to non-responders after three weeks. The selection of participants is performed in two cycles for practical reasons. The first cycle started in December 2009 and the second in February 2010. The undertaking of the intervention and the collection of data will be performed in two cycles as well.

\section{Randomisation}

Cluster randomisation was applied to avoid contamination bias. ${ }^{24-26}$ Before the screening procedure started, six practices were randomly allocated to the new intervention and six practices continued care as usual. Before randomisation, the GP practices were pre-stratified into four strata on the basis of practice characteristics, which may influence the results of the study:

1. number of older patients in the practice ( $<350$ patients versus $\geq 350$ patients);

2. urban versus rural area.

It is assumed that GPs working in a practice with a large number of older patients have more experience with geriatric care. Furthermore, it is expected that olde people living in a rural area have more support from the informal care system than people living in an urban area.

The practices were stratified in pairs and randomised into either intervention or contro group using a computer generated randomisation list. To promote extrapolation of the results, practices settled in an urban area with a large number of older people had twice the chance of being included in the study than practices in the other three strata.

Intervention and control group

Intervention group

Based on literature studies and an expert meeting a first draft of the intervention protocol was developed by a multidisciplinary task group. This group consisted of a GP, a nursing home physician, a geriatrician, a practice nurse, a home nurse staf member, a nurse specialist, a physical therapist, an occupational therapist, an exper in technology and a researcher as the coordinator. Studies on the screening procedure ${ }^{27}$ and on the validity of screening instrument ${ }^{28}$ were followed by a pre-pilot study (one GP practice, 10 frail older people) and a pilot study (two GP practices, 50 frail older people $)^{29}$ to test the feasibility of the intervention programme. The results were used to develop the final version of the intervention protocol under study.
The GP and the practice nurse are the core team of the intervention with the practice nurse as the case manager. This core team can be extended to include an occupational therapist and a physiotherapist, or other inpatient and outpatient specialists. The intervention puts emphasis on supporting frail older people to restore or continue the activities they need or enjoy, assuming that participation in social and productive activities is protective against adverse outcomes. ${ }^{29}$ The intervention has two main features:

- Identifying risk factors ${ }^{30}$ for developing disability and targeting risk factors using professional standards (i.e. Standards of Dutch College of GPs) and the $5 \mathrm{~A}$ Behavioural Change Model. ${ }^{31}$

- Identifying problems in performing activities and enhancing meaningful activities based on the Model of Human Occupation. ${ }^{32}$

The intervention takes an individual approach to self-management. The 5A Behavioural Change Model combines a client-centred approach, a model for behavioural change (Stages of Change) and motivational interviewing techniques to provide concrete tools for professionals to support self-management. The 5As refer to the assessment of levels of behaviour, beliefs and motivation, advice adapted to the need for information, the agreement with frail older people on a realistic set of goals, the assistance provided to help them to anticipate barriers and the development of a specific action plan and arrangement of follow-up support. ${ }^{31}$ In terms of performing activities, the Model of Human Occupation ${ }^{32}$ is considered to be a good tool for analysis and problem-solving. With its base in occupational therapy, the model illuminates how factors of capacity, motivation, lifestyle and environment inter-relate in human occupation. This model has been used in previous successful effect studies. 33,34

The intervention consists of six steps (see Figure 2). After an initial posta screening (step 1) frail older people (GFI score 25 ) will receive a comprehensive multidimensional assessment (step 2) by a practice nurse in collaboration with the GP. This assessment phase will focus on the identification of existing problems in performing daily activities and on risk factors for developing disability (i.e. poly-pharmacy, mobility, lack of social and productive activities, cognitive impairments). The practice nurse and GP will determine if additional assessment is needed by the GP physiotherapist, occupational therapist or other inpatient or outpatient specialists. At the end of the assessment phase the practice nurse and GP will develop an intermediate action plan (step 3). Alternatively in cases of complex problems, the interdisciplinary team (i.e. practice nurse, GP, physiotherapist and occupational therapist) will meet to formulate a shared action plan. Consequently, a meeting between the practice nurse and the frail older person (and informal caregiver) will take place to define a final action plan, including goals, strategies and actions (step 4). The action plan will be tailored to the specific needs and wishes of the frail older person and will be related to a flexible toolbox of interventions which will be conducted 
Screening: GP practice sends GFI to older persons $(\geq 70)$. GFI score $\geq 5$ : PN calls for home visit.

\section{Assessment: home vis}

(and informal caregiver) ollowed by decision making with GP about necessity for:

Assessment OT and/ or PT (advised in case of concerns or problems in performing activities).

Additional assessments

Analyses and action plan: Perspective GP and PN (or extended team including OT, PT and others) on current problems in performing

activities and risk factors for developing disability.

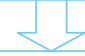

Agreement on action plan: home visit

PN negotiates with older person (and informal caregiver) on goals, actions and toolbox parts.

\section{Execution of action plan}

Meaningful activities(OT): increasing client's awareness activities.

2. Adaptation of environment, skills or activities (PT and OT): adapting the environment (e.g. technology), learning new skills or new ways of performing activities. A tailormade physical exercise programme can be applied in case improvements in strength, balance, flexibility and endurance are believed to contribute to reducing the risk of disability.

3. Social network and social activities (PN): organising the social network and resources to fulfil needs for social

4. Physical activity (PT): increasing physical activity in

5. Stimulate health (GP and PN): measures that will increase health and maintenance of a healthy lifestyle.

Other interventions can be applied besides the toolbox $\mathrm{PN}$ is case manager monitoring progress and satisfaction

\section{Evaluation and follow-up:}

$\mathrm{PN}$ and older person (and caregiver) evaluate progress and agree

on follow-up

\section{Figure 2 Steps of the programme.}

$\mathrm{PN}=$ practice nurse, $\mathrm{GP}=$ general practitioner, $\mathrm{OT}=$ occupational therapist, $\mathrm{PT}=$ physiotherapis by the interdisciplinary team (step 5). The toolbox can be supplemented with other interventions delivered by inpatient and outpatient specialists. The intervention protocol provides guidelines for referral to other disciplines. During execution and after finishing the components of the toolbox, the practice nurse will evaluate, with the frail older person (and the informal caregiver), the achievement of goals, the implementation of strategies into daily life and the need for further support (step 6). ${ }^{29}$

Training of healthcare professionals

In the 3-month period before the start of the intervention, healthcare professionals (practice nurses, GPs, physiotherapists and occupational therapists) received relevant training sessions with regard to the intervention protocol. Several meetings about the aspects and basic principles of the intervention protocol took place (i.e. the screening procedure, self-management principles, client centeredness, motivational interviewing, interdisciplinary collaboration, assessment tools, parts of the toolbox and referrals). Before the start of the study, health professionals had the opportunity to gain experience with the intervention protocol under supervision by the project team in small samples of frail older people who were not included in the study.

Control group

Frail older people in the control group were also selected by means of the screening questionnaire (GFI score 25 ). They will receive care as usual, and will be able to use or apply for all available services for older people as before.

Outcome

Primary outcome measure

The primary outcome measure is disability. The Groningen Activity Restriction Scale (GARS) is an easy-to-administer, comprehensive, reliable, hierarchical and valid measure for assessing disability in the domains of activities of daily living (ADL) and instrumental activities of daily living (IADL) in older people. ${ }^{35}$

Secondary outcome measures

Secondary outcomes are depressive symptomatology (depression subscale Hospita Anxiety and Depression Scale) ${ }^{36}$, social support interactions (Social Support List Interaction version) $)^{37}$, fear of falling (Short Falls Efficacy Scale - International) ${ }^{38}$ and social participation (Maastricht Social Participation Profile) ${ }^{39}$. Feelings of loneliness will be assessed by the question: "During the past 4 weeks, how often did you fee lonely?". ${ }^{9}$ The frequency of falls will be assessed by the question: "How often did you fall during the past 6 months/ 12 months". ${ }^{19}$ Mortality, healthcare utilisation and related costs will be continuously registered during 24 months. 
Additional variables

Several additional variables will be assessed to provide insight into population characteristics and to interpret the outcomes of the study. Mental, physical, social, economic and behavioural factors may affect our primary outcome of disability. ${ }^{4}$ Socio-demographic data (i.e. age, gender, marital status, living situation, educationa level) will be gathered at baseline from the GFI and a standardised data set: the Minimal DataSet (MDS). Other variables to be assessed at baseline and at 6,12 and 24 month follow-up are cognitive impairment ${ }^{41}$ and vision and hearing capacity ${ }^{42}$.

We assume two variables to be potential effect modifiers. First, the frailty status at baseline (GFI score) as it predicts disability. ${ }^{5}$ Second, feelings of competence and control, as they are crucial for self-management and coping ${ }^{43}$, which are importan underlying mechanisms of the proposed intervention. Feelings of competence and control will be assessed at baseline and at 6,12 and 24 month follow-up using the Mastery scale. ${ }^{44}$ The impact of potential effect modifiers on disability will be studied in subgroup analyses. Table 1 provides an overview of the instruments used to measure primary and secondary outcomes, and the additional variables.

The proposed study will be embedded in the Dutch National Care for the Elderly Programme. This implies that the MDS has to be applied. The MDS for the care receiver provides global data on: age, gender, marital status, ethnicity, living arrangements, socio-economic status, level of education, health perception, multimorbidity, daily functioning in ADL, mental well-being, cognitive functioning, social functioning, quality of life and use of healthcare services. Data about the impact of the intervention on informal caregivers (perceived burden and health-related quality of life) will be gathered by the MDS for informal caregivers.

Data will be collected by a combination of two methods, telephone interviews (TI) and postal questionnaires (PQ), which have been proven to be feasible and efficient in previous research. ${ }^{18,19}$ First, a second postal questionnaire after the screening questionnaire will be sent to frail older people to gather baseline data. Two weeks later a telephone interview will take place in order to gather addition information. These interviews will be conducted by independent interviewers of the Centre for Data and Information Management of Maastricht University (MEMIC), who will be blinded to the treatment assignment. The same procedure (postal questionnaire and telephone interview) will be repeated after 6,12 and 24 months after baseline to gather follow-up data. In addition, data on healthcare utilisation and related costs wil be continuously gathered from health insurance registries and registries of the GPs and hospitals in the region. The central informal caregivers of the frail older people will receive a questionnaire at baseline and after 6, 12 and 24 months.
Table 1 Primary, secondary and additional outcome measures.

\begin{tabular}{|c|c|c|c|c|c|c|c|}
\hline Variables & Instrument & $\begin{array}{l}\text { No. of } \\
\text { items }\end{array}$ & Range* $^{*}$ & B & FU1 & FU2 & FU3 \\
\hline \multicolumn{8}{|l|}{ Primary outcome measure } \\
\hline Disability & GARS $^{35}$ & 18 & $\underline{18-72}$ & TI & $\mathrm{TI}$ & $\mathrm{TI}$ & $\mathrm{TI}$ \\
\hline \multicolumn{8}{|c|}{ Secondary outcome measures } \\
\hline Cognitive impairment & TICS ${ }^{41}$ & 11 & $0-\underline{41}$ & TI & $\mathrm{Tl}$ & $\mathrm{Tl}$ & $\mathrm{TI}$ \\
\hline $\begin{array}{l}\text { Symptoms of } \\
\text { depression }\end{array}$ & $\operatorname{HADS}^{36}$ & 7 & $\underline{0}-21$ & TI & $\mathrm{Tl}$ & $\mathrm{TI}$ & $\mathrm{Tl}$ \\
\hline Social participation & MSPPз9 & 10 & $0-\underline{90}$ & TI & $\mathrm{Tl}$ & $\mathrm{TI}$ & $\mathrm{Tl}$ \\
\hline $\begin{array}{l}\text { Social support } \\
\text { interactions }\end{array}$ & SSL12- $\left.\right|^{37}$ & 12 & $12-\underline{48}$ & $P Q$ & $P Q$ & $P Q$ & $P Q$ \\
\hline Fear of falling & Short FES-138 & 7 & 7-28 & TI & $\mathrm{TI}$ & $\mathrm{TI}$ & $\mathrm{TI}$ \\
\hline $\begin{array}{l}\text { No. of falls in the } \\
\text { previous } 6 \text { months }\end{array}$ & $N / A$ & 1 & $\mathrm{~N} / \mathrm{A}$ & TI & $\mathrm{Tl}$ & $\mathrm{TI}$ & $\mathrm{TI}$ \\
\hline $\begin{array}{l}\text { Consultation with } \\
\text { physician due to fall }\end{array}$ & N/A & 1 & N/A & TI & $\mathrm{TI}$ & $\mathrm{TI}$ & $\mathrm{TI}$ \\
\hline Feelings of loneliness ${ }^{19}$ & $N / A$ & 1 & $\mathrm{~N} / \mathrm{A}$ & $P Q$ & $P Q$ & $P Q$ & $P Q$ \\
\hline Mortality & N/A & N/A & N/A & $\mathrm{R}$ & $\mathrm{R}$ & $\mathrm{R}$ & $\mathrm{R}$ \\
\hline \multicolumn{8}{|l|}{ Additional measures } \\
\hline $\begin{array}{l}\text { Vision/hearing } \\
\text { capacity }\end{array}$ & $\begin{array}{l}\text { OECD-long-term } \\
\text { disability indicator }{ }^{42}\end{array}$ & 4 & $4-16$ & $\mathrm{TI}$ & $\mathrm{Tl}$ & $\mathrm{TI}$ & $\mathrm{TI}$ \\
\hline Mastery & Mastery scale ${ }^{44}$ & 7 & $7-\underline{35}$ & $\mathrm{TI}$ & $\mathrm{Tl}$ & $\mathrm{Tl}$ & $\mathrm{TI}$ \\
\hline Healthcare utilisation & N/A & N/A & N/A & $\mathrm{R}$ & $\mathrm{R}$ & $\mathrm{R}$ & $\mathrm{R}$ \\
\hline
\end{tabular}

* The underlined scores indicate the most favourable scores; $N / A=$ not applicable

B=baseline; FU1=6-month follow-up; FU2=12-month follow-up; FU3=24-month follow-up $\mathrm{Tl}=$ =elephone interview, $\mathrm{PQ}=$ postal questionnaire; $\mathrm{R}=$ registries of health insurance/ $\mathrm{GP} /$ hospita

Process evaluation

The process evaluation aims to improve further implementation of the intervention and to validate the results of the study. A systematic approach will be used for this evaluation, involving key elements such as reach, dose delivered, dose received (exposure and satisfaction) and barriers. ${ }^{45-47}$ Table 2 gives a description of these elements. 
Table 2 Elements of the process evaluation and data collection methods

\begin{tabular}{|c|c|c|}
\hline Component and definition & Outcome variables & Measurement \\
\hline \multicolumn{3}{|l|}{ Reach } \\
\hline $\begin{array}{l}\text { Proportion of the intended } \\
\text { target population that } \\
\text { participated in the } \\
\text { intervention }\end{array}$ & $\begin{array}{l}\text { - Number of older people that refused, } \\
\text { dropped-out or completed the } \\
\text { intervention } \\
\text { - Reasons for refusal/drop-out (before } \\
\text { start and during the intervention) }\end{array}$ & - Logbook \\
\hline \multicolumn{3}{|l|}{ Dose delivered (completeness) } \\
\hline $\begin{array}{l}\text { Amount of delivered } \\
\text { intervention }\end{array}$ & $\begin{array}{l}\text { - Assessments } \\
\text { - Referrals to other disciplines } \\
\text { - Interventions / toolbox parts } \\
\text { - Evaluation / follow-up }\end{array}$ & - Logbook \\
\hline \multicolumn{3}{|l|}{ Dose received (exposure) } \\
\hline $\begin{array}{l}\text { Extent of active } \\
\text { engagement in and } \\
\text { receptiveness to the } \\
\text { intervention by older people }\end{array}$ & $\begin{array}{l}\text { - Opinion about older peoples' ability } \\
\text { to understand and implement } \\
\text { principles of the intervention } \\
\text { - Adherence to commitments made by } \\
\text { older people } \\
\text { - Intention of patients to implement the } \\
\text { intervention }\end{array}$ & $\begin{array}{l}\text { - Questionnaire/ } \\
\text { interviews older } \\
\text { people } \\
\text { - Questionnaire/ } \\
\text { interviews } \\
\text { healthcare } \\
\text { professionals }\end{array}$ \\
\hline \multicolumn{3}{|l|}{ Dose received (satisfaction) } \\
\hline $\begin{array}{l}\text { Satisfaction of older } \\
\text { people and healthcare } \\
\text { professionals with the } \\
\text { intervention }\end{array}$ & $\begin{array}{l}\text { - Overall opinion of older people } \\
\text { - Experienced benefits, burden, } \\
\text { usefulness by older people } \\
\text { - Overall opinion of healthcare } \\
\text { professionals }\end{array}$ & $\begin{array}{l}\text { - Questionnaire/ } \\
\text { interviews older } \\
\text { people } \\
\text { - Questionnaire/ } \\
\text { interviews } \\
\text { healthcare } \\
\text { professionals }\end{array}$ \\
\hline \multicolumn{3}{|l|}{ Barriers } \\
\hline $\begin{array}{l}\text { The extent to which } \\
\text { problems were } \\
\text { encountered while applying } \\
\text { the intervention }\end{array}$ & - Barriers in applying the intervention & $\begin{array}{l}\text { - Questionnaire/ } \\
\text { interviews } \\
\text { healthcare } \\
\text { professionals }\end{array}$ \\
\hline
\end{tabular}

Both quantitative and qualitative information will be collected from participants and healthcare professionals. Participants will evaluate the intervention by means of a selfadministered questionnaire, directly after their last intervention contact. In addition, semistructured interviews will be conducted among a random sample of participants to evaluate their experiences with the intervention. Questionnaires and semi-structured

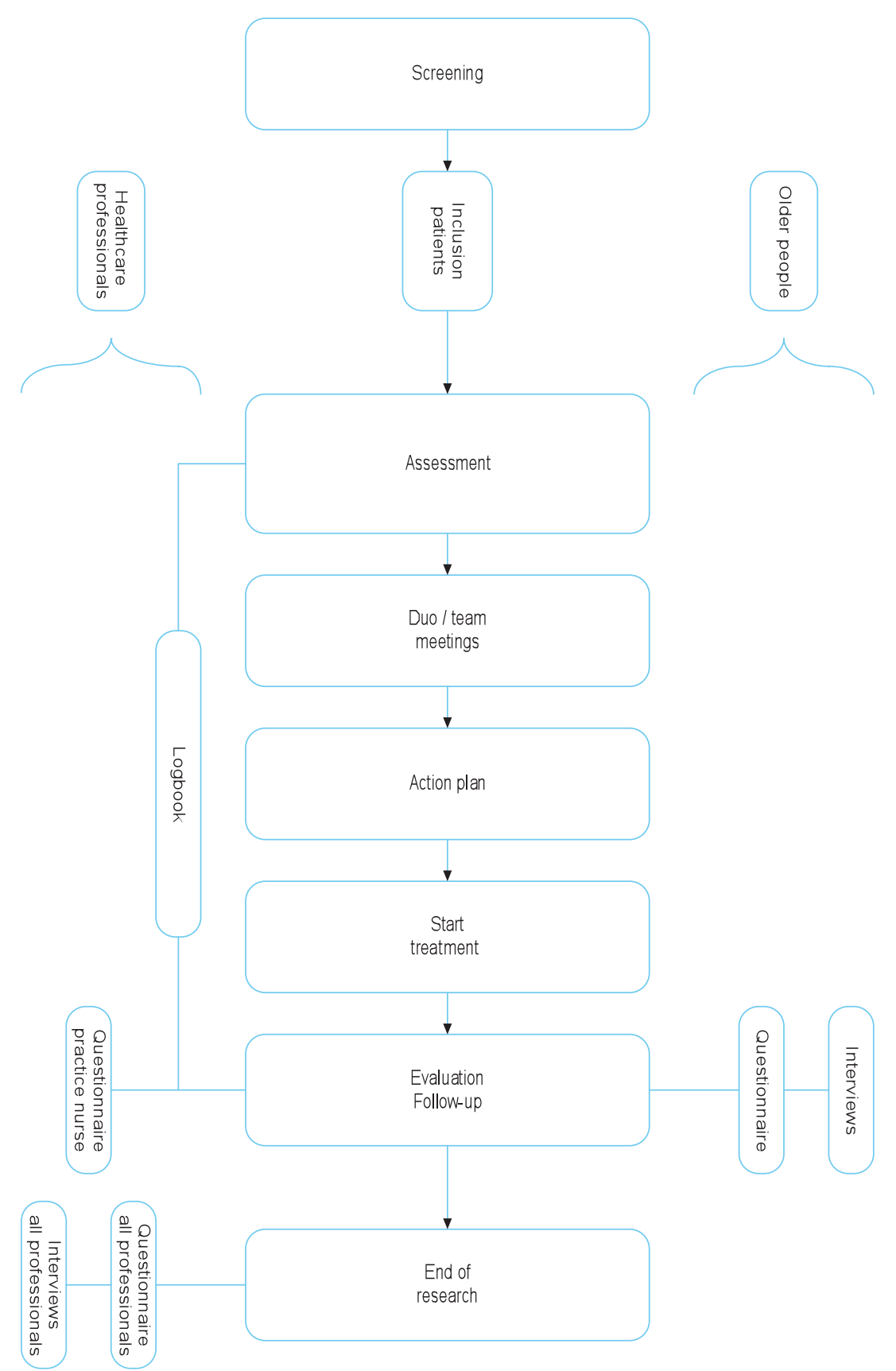

Figure 3 Process evaluation plan. 
interviews will also be used among healthcare professionals to evaluate the intervention. In addition, they will be asked to register the treatments delivered (including the time spent on them) and reasons given for refusal and dropout throughout the intervention period. Figure 3 shows the data to be collected for the process evaluation during the study period.

Economic evaluation

A cost-effectiveness analysis will be carried out in which costs will be considered from a societal perspective, which implies that all costs and effects are taken into account. The economic evaluation will be a combination of a cost-effectiveness and cost-utility analysis. The primary clinical outcome for the cost-effectiveness analysis is disability, which will be measured at baseline and at 6,12 and 24 months follow-up by means of the GARS. ${ }^{35}$ Within the cost-utility analysis, the primary outcome is generic health-related quality of life (QALY). Therefore utilities will be measured by means of the standard Dutch version of the EuroQol (EQ-6D) $)^{48,49}$ at baseline and at 6 , 12 and 24 months follow-up. Subsequently, a direct value for every state of health will be generated using the Dolan tariff, which involves an algorithm for interpolating EuroQol results to population utilities. .0,51 $^{\circ}$

This study will assess intervention costs, healthcare costs and patient/ family costs. Intervention costs relate to the delivery of the intervention, for example costs related to the screening procedure, time spend on intervention by healthcare professionals, travel expenses of professionals and costs for training activities. Data will be registered prospectively by the researchers. Healthcare costs relate to hospita visits (inpatient and outpatient treatment), GP consultations, visits to allied professionals, prescribed medication and (nursing) home care. Data of healthcare utilisation will be assessed by means of registries of health insurance agencies, GP practices and hospitals. In addition, questions about healthcare utilisation are included in the postal questionnaire and the telephone interview. Patient and family costs relate to costs that are made by the patients or informal caregivers themselves and include, for example, travel expenses (based on the mean distance to and from healthcare professionals) and informal care (based on hours delivered). These data would preferably be assessed by means of a cost diary. ${ }^{52}$ However, in the present study a diary was considered to be too burdensome for frail older people (and thei informal caregivers).

In order to calculate costs, volumes of resource utilisation will be multiplied by the cost price of that unit. Cost prices will be obtained from the Dutch guidelines for cost analysis in health care research. ${ }^{53,54}$ Where such guidelines are not available for a specific category, real costs or tariffs will be used to estimate costs.

Differences in costs and effects will be presented in incremental cost-effectiveness ratios (ICERs). The ICERs represent the differences in mean costs between the intervention and usual care group in the numerator and the difference in mean effects in the denominator. ${ }^{55}$ Sensitivity analysis will be done to assess the robustness of the assumptions we made.

Sample size

The population sample size is based on the primary outcome measure of disability Based on a previous study ${ }^{19}$, we expect to demonstrate a difference in disability between the mean change score of the intervention and the control group of at least 2.0 points on the Groningen Activity Restriction Scale (GARS) ${ }^{35}$ (which is equivalen to an effect size of 0.44 with SD 4.5). Based on a power of $80 \%$ and an alpha of 0.05 (two-sided testing), this leads to a minimal sample size of $n=80$ per group (160 in total). Based on an expected drop-out rate of $30 \%$, the required sample size would be $n=104$ per group ( $n=208$ in total). However, the cluster randomised design of this study has consequences for the sample size and power. Scores of individuals within a cluster are assumed to be correlated in contrast to those of individuals between clusters. A within-cluster correlation leads to a greater homogeneity of individuals within a cluster, which increases the standard error of the estimate of the treatment effect. This may result in a loss of power for detecting differences between the intervention and control group. Therefore, an Intra Cluster Correlation (ICC) coefficient is needed to determine a corrected sample size. ${ }^{56}$ In earlier intervention trials among GPs, ICC values between 0.03 and 0.06 were used. ${ }^{57-59}$ In the present study an ICC value of 0.05 was estimated, resulting in a design effect of 1.7273 . Based on the expected drop-out rate of $30 \%$, the required sample size is $n=180$ per group $(n=360$ in total, an average of $\mathrm{n}=30$ per GP practice).

According to Puts and colleagues (2005), the prevalence of frailty in Dutch older people aged 55 to 85 years old varies from $12 \%$ to $21 \% .{ }^{60}$ We have conservatively estimated that $15 \%$ of the screened population will fulfil all criteria. A response rate on the postal screening of at least $65 \%$ was expected among older people..$^{19,61}$ On average, 300 screening questionnaires per GP practice (3,498 in total) were sent to older people to obtain a sufficient number of frail older people for the trial $(n=360)$. In addition, (central) informal caregivers will be included as well. It is expected that a central informal caregiver will be identified for $80 \%$ of frail older people.

Statistical analysis

Descriptive techniques will be used to describe the study groups. Baseline variables will be compared to detect differences between the intervention and control group a the start of the study. Data of the effect evaluation will be analysed according to the intention-to-treat principle. Analysis of primary and secondary endpoints will be performed using relevant univariate, multivariate and multilevel techniques including mixed-effects regression models. A subgroup analysis will be performed for frailty status and feelings of competence and control (mastery), as these variables are 
assumed to be potential effect modifiers. The software package SPSS for Windows, version 17.0., will be used for all statistical analyses. The level of statistical significance will be set at 0.05 (two-tailed)

Data on the process and economic evaluation will be analysed and presented using descriptive techniques and appropriate statistical testing. Data gathered from interviews (process evaluation) will be analysed using descriptive techniques.

\section{Discussion}

Out of 52 GP practices, 24 practices have applied for taking part in the proposed study. This indicates a substantial interest of GPs in innovations regarding care fo frail older people. Twelve of them were randomly selected to take part in the current study. A random selection of their community-dwelling older patients ( $\geq 70$ years) was screened for frailty. For practical reasons the screening procedure was distributed across two cycles between December 2009 and April 2010. A total of 3,498 older people received the screening questionnaire. The response rate was $80 \%$. According to the GFI scores $29 \%$ of the respondents can be considered as frail (GFI score $\geq 5$ ). Nearly half of them (48\%) were willing to participate. Sending out a postal questionnaire including the GF| ${ }^{22}$ turned out to be a feasible and inexpensive method of identifying frail older people. Adding a letter from their general practitioners to the information leaflet may have contributed to the high response rate.

The proposed study has some potential limitations. First, the participating GPs are very interested in innovations in the care of older people otherwise they would not have applied to take part in the study. Consequently, the GPs allocated to the contro group may take initiatives to improve the care for the older people in this group during the study period. The researchers will carefully monitor the activities of GPs regarding the potential improvement of care of these older people. Second, implementation of the intervention protocol is a point of concern. Based on a combination of elements such as interdisciplinary decision-making and collaboration (i.e. team meetings) self-management (taking principles of client-centeredness, behavioural change and motivational interviewing into account), and an extensive toolbox of interventions, the intervention is very complex. Educating and guiding teams in implementing the programme are therefore important.

\section{Progress of study}

The baseline measurements started in January 2010. In February 2010 the first frai older people were approached by the PN for a comprehensive assessment. Data on the effect evaluation will be available in 2012. Data for the process evaluation and economic evaluation will be gathered between 2010 and 2012.
Acknowledgements

- Participants of the study

Participating GPs and other healthcare professionals, who are involved in the research;

- Health Insurance companies

- Centre for Data and Information Management (MEMIC): Anita Legtenberg, Alfons Schroten, Marlène Ronner

- Members of the research group: Sjoerd Hobma (GP), Lilo Crasborn (MCC Omnes), Simone Denis (MCC Omnes), Marlou Wolters (MCC Omnes), Herbert Habets (Orbis Medical Centre), Walther Sipers (Orbis Medical Centre), Ramon Daniels (Zuyd University of Applied Sciences, Heerlen);

- Research assistance: Floor Koomen, Ine Hesdahl.

- Sponsor: Dutch National Care for the Elderly Programme by The Netherlands Organisation for Health Research and Development (ZonMw 311070301), the Hague, the Netherlands. 


\section{References}

. Slaets JPJ. Vulnerability in the elderly: frailty. Med Clin North Am. 2006;90(4):593-601.

2. Levers MJ, Estabrooks CA, Ross Kerr JC. Factors contributing to frailty: literature review. J Adv Nurs 2006;56(3):282-91.

3. Markle-Reid M, Browne G. Conceptualizations of frailty in relation to older adults. I Adv Nurs 2003;44(1):58-68

4. Pel Littel RE, Schuurmans MJ, Emmelot Vonk MH, Verhaar HJJ. Frailty: defining and measuring of a concept. J Nutr Health Aging. 2009;13(4):390-4.

5. Fried LP, Ferrucci L, Darer J, Williamson JD, Anderson G. Untangling the concepts of disability, frailty, and comorbidity: implications for improved targeting and care. Gerontol A Biol Sci Med Sci 2004;59(3):255-63.

6. Keating N, Ottinowski P, Wenger C, Fast J, Derksen L. Understanding the caring capacity of informal (1)

7. Ferrucci L, Guralnik JM, Studenski S, Fried LP, Cutler GB, Jr., Walston JD. Designing randomized, controlled trials aimed at preventing or delaying functional decline and disability in frail, older persons: consensus report. J Am Geriatr Soc. 2004:52(4):625-34.

8. Daniels R, Metzelthin S, Van Rossum E, De Witte L, Van den Heuvel W. Interventions to prevent disability in frail community-dwelling older persons: an overview Eur J Ageing 2010;7(1):37-55.

9. Rubenstein LZ, Siu AL, Wieland D. Comprehensive geriatric assessment: toward understanding its efficacy. Aging (Milano). 1989;1(2):87-98

10. Beswick AD, Rees K, Dieppe P. Ayis S, Gooberman-Hill R, Horwood J, Ebrahim S. Complex interventions to improve physical function and maintain independent living in elderly people: a systematic review and meta-analysis. Lancet. 2008;371(9614):725-35

11. Wieland $\mathrm{D}$. The effectiveness and costs of comprehensive geriatric evaluation and management. $\mathrm{Cr}$ Rev Oncol Hematol. 2003;48(2):227-37.

12. Wieland D, Hirth V. Comprehensive geriatric assessment. Cancer Control. 2003;10(6):454-62

13. Daniels $R$, van Rossum $E$, de Witte $L$, Kempen GI, van den Heuvel W. Interventions to prevent disability in frail community-dwelling elderly: a systematic review. BMC Health Serv Res. 2008;8(1):278

14. Mann WC, Ottenbacher KJ, Fraas L, Tomita M, Granger CV. Effectiveness of assistive technology and environmental interventions in maintaining independence and reducing home care costs for the fra elderly. A randomized controlled trial. Arch Fam Med. 1999;8(3):210-7.

15. Tomita MR, Mann WC, Stanton K, Tomita AD, Sundar V. Use of currently available smart home technology for frail elders; process and outcomes. Top Geriatr Rehabil 2007;23(1):24-34.

6. Frieswijk N, Steverink N, Buunk BP, Slaets JPJ. The effectiveness of a bibliotherapy in increasing the selmanagement ability of slightly to moderately frail older people. Patient Educ Couns. 2006;61(2):219-27.

17. Lamers F, Jonkers CCM, Bosma H, Kempen GIJM, Meijer JAMJ, Penninx BWJH, Knottnerus JA, van Eik JTM. A minimal psychological intervention in chronically ill elderly patients with depression: a randomized trial. Psychother Psychosom. 2010;79:217-26.

8. Smeulders ESTF. Heatt failure sell-management. balancing between medical and psychosocial needs. Evaluation of a cognitive-behavioural self-management group programme $[\mathrm{PhD}$ thesis]. Maastrich School for Public Health and Primary Care, Maastricht University; 2009.

19. Zijlstra GAR. Managing concerns about falls. Fear of falling and avoidance of activity in older people. [PhD thesis]. Maastricht: School for Public Health and Primary Care, Maastricht University; 2008.

20. Fried LP, Carlson MC, Freedman M, Frick KD, Glass TA, Hill J, McGill S, Rebok GW, Seeman T, Tielsch J, Wasik BA, Zeger S. A social model for health promotion for an aging population: initial evidence on the Experience Corps model. J Urban Health. 2004;81(1):64-78.

21. Graff MJ, Vernooij-Dassen MJ, Thijssen M, Dekker J, Hoefnagels WH, Olde Rikkert MG. Community based occupational therapy for patients with dementia and their care givers: randomised controlled tria. BMJ. 2006;333(7580):1196.

22. Steverink N, Slaets JPJ, Schuurmans H, van Lis M. Measuring Frailty: development and testing of the Groningen Frailty Indicator (GFI). Gerontologist. 2001;41(1):236-7.
23. Schuurmans H, Steverink N, Lindenberg S, Frieswijk N, Slaets JPJ. Old or frail: what tells us more? J Gerontol A Biol Sci Med Sci. 2004;59(9):M962-5.

24. Campbell MK, Elbourne DR, Altman DG, and CONSORT group. CONSORT statement: extension to cluster randomised trials. BMJ. 2004;328(7441):702-8.

25. Campbell MK, Grimshaw JM. Cluster randomised trials: time for improvement. The implications of adopting a cluster design are still largely being ignored. BMJ. 1998;317(7167):1171-2.

26. Eldridge S, Ashby D, Bennett C, Wakelin M. Feder G. Internal and external validity of cluster randomised trials: systematic review of recent trials. BMJ. 2008;336(7649):876-80.

27. Steinbusch C. Screening of frail elderly in the community. The feasibility and psychometric properties three instruments [MSc thesis]. Maastricht. School for Public Health and Primary Care Mastricht: 2008

Metzelthin SF Danies R, van Rossum E de Witte L, van den Heuel WJ Kempen GI The psychometric for identifyng frail older people living in the properties or the selvepth screning instruments tor identying tral or

29. Daniels R. Frail Elderly - Identification and disability prevention in primary care [PhD Thesis]. Maastricht School for Public Health and Primary Care, Maastricht University; 2011.

30. Stuck AE, Walthert JM, Nikolaus T, Bula CJ, Hohmann C, Beck JC. Risk factors for functional status decline in community-living elderly people: a systematic literature review. Soc Sci Med. 1999;48(4):445-69

31. Bodenheimer T, Grumbach K. Improving primary care. Strategies and tools for better practice. New York Lange Medical Books/McGraw-Hill; 2007.

32. Kielhofner G. Model of Human Occupation. Theory and application. Baltimore: Kluwer/Lippincot Williams\&Wilkins Wolters; 2008

33. Corr S, Bayer A. Occupational therapy for stroke patient after hospital discharge - a randomized controlled trial. Clin Rehabil 1995;9:291-6.

34. Graff MJ, Vernooii-Dassen MJ, Thijssen M, Dekker J, Hoefnagels WH, Olde Rikkert MG. Effects of community occupational therapy on quality of life, mood, and health status in dementia patients and their caregivers: a randomized controlled trial. J Gerontol A Biol Sci Med Sci. 2007;62(9):1002-9.

35. Kempen GIJM, Miedema I, Ormel J, Molenaar W. The assessment of disability with the Groningen Activity Restriction Scale. Conceptual framework and psychometric properties. Soc Sci Med. 1996;43(11): $1601-10$

36. Spinhoven P, Ormel J, Sloekers PPA, Kempen GIJM, Speckens AEM, van Hemert AM. A validation study of the Hospital Anxiety and Depression Scale (HADS) in different groups of Dutch subjects. Psycho/ Med. 1997;27(2):363-70

37. Kempen GIJM, van Eijk LM. The psychometric properties of the SSL12-I, a short scale for measuring social support in the elderly. Soc Indic Res 1995;35(3):303-12.

38. Kempen GIJM, Yardley L, van Haastregt JCM, Zijlstra GAR, Beyer N, Hauer K, Todd C. The Short FESa shortened version of the falls efficacy scale-international to assess fear of falling. Age Ageing 2008; $37(1): 45-50$.

39. Mars GMJ, Kempen GIJM, Post MWM, Proot IM, Mesters I, van Eijk JTM. The Maastricht socia participation profile: development and clinimetric properties in older adults with a chronic physica illness. Qual Life Res. 2009;18(9):1207-18.

40. Avila-Funes JA, Helmer C, Amieva H, Barberger-Gateau P, Le Goff M, Ritchie K, Portet F, Carriere Tavernier B, Gutierrez-Robledo LM, Dartigues JF. Frailty among community-dwelling elderly people in France: the three-city study. J Gerontol A Biol Sci Med Sci. 2008;63(10):1089-96.

41. Kempen Gl, Meier AJ, Bouwens SF, van Deursen J, Verhey FR. The psychometric properties of the Dutch version of the Telephone Interview Cognitive Status (TICS) [Article in Dutch]. Tijdschr Gerontol Geriatr 2007;38(1):38-45.

42. Van Sonsbeek JLA. Methodological aspects and content of the OECD-lonterm disability indicator. CBS Maandberichten Gezondheid. 1988;6:4-17.

33. Kempen GIJM. Preventie en ouder worden: mogelijkheden voor zelfmanagement. Epid Bull. 2006;41(2):27-32.

44. Pearlin LI, Schooler C. The structure of coping. J Health Soc Behav. 1978;19(1):2-21.

45. Baranowski T, Stables G. Process evaluations of the 5-a-day projects. Health Educ Behav. 2000;27(2):157-66. 
46. Linnan L, Steckler AB. Process evaluation for public health interventions and research: an overview. Process evaluation for public health interventions and research. San Francisco: Jossey-Bass; 2002:1-23.

4. Saunders RP. Evans MH, Joshi P. Developing a process-evaluation plan for assessing health promotion program implementation: a how-to guide. Health Promot Pract. 2005;6(2):134-47.

48. Krabbe PFM, Stouthard MEA, Essink-Bot M-L, Bonsel GJ. The effect of adding a cognitive dimension to the EuroQol multiattribute health-status classification system. J Clin Epidemiol. 1999;52(4):293-301:

49. Wolfs CA, Dirksen CD, Kessels A, Willems DC, Verhey FR, Severens JL. Performance of the EQ-5D and the EQ-5D+C in elderly patients with cognitive impairments. Health Qual Life Outcomes. 2007:5:33.

50. Dolan P. Modelling valuations for EuroQol health states. Med Care 1997;35:1095-108.

51. Hoeymans $\mathrm{N}$, van Lindert $\mathrm{H}$, Westert GP. The health status of the Dutch population as assessed by the EQ-6D. Qual Life Res. 2005;14(3):655-63.

52. Goossens ME, Rutten-van Molken MP, Vlaeyen JW, van der Linden SM. The cost diary: a method to measure direct and indirect costs in cost-effectiveness research. J Clin Epidemiol. 2000;53(7):688-95.

53. Oostenbrink JB, Bouwmans CAM, Koopmanschap MA, Rutten FFH. Manual for cost-analysis in healthcare research: The Health Care Insurance Board; 2004.

54. Oostenbrink JB, Koopmanschap MA, Rutten FF. Standardisation of costs: the Dutch manual for costing in economic evaluations. Pharmacoeconomics. 2002;20(7):443-54

55. Drummond MF, Sculpher MJ, Thorrance GW, O'Brien BJ, Stoddard GL. Methods for the economic evaluation of health care programmes. 3rd edition ed. New York: Oxford University Press; 2005.

56. Parker DR, Evangelou E, Eaton CB. Intraclass correlation coefficients for cluster randomized trials in primary care. the cholesterol education and research thal (CEART). Contemp Cin Thals. 2005,20(2).260-7.

57. Koelewinn-Van Loon MS, van Steenkiste B, Ronda G, Wensing M, Storers HE, Elwyn G, Grol R, van der Weijden T. Improving palion adhoronce to litestyle advice (MPALA). a clusterrandomised controlled

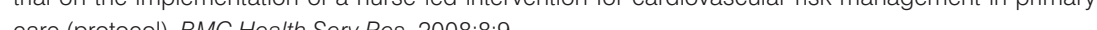
cal (procol). BMCHealh Sen Res. $2008,89$.

58. Van der Weijden T, Grol RP, Knottnerus JA. Feasibility of a national cholesterol guideline in daily practice. A randomized controlled trial in 20 general practices. Int J Qual Health Care. 1999;11(2): 131-7.

59. Van Steenkiste B, van der Weijden T, Stoffers HE, Kester AD, Timmermans DR, Grol R. Improving cardiovascular risk management: a randomized, controlled trial on the effect of a decision support too Tor palients and physicians. Eur Cardiovasc Prev Rehabil. 2007,14(1):44-50.

60. Puts MT, Lips P, Deeg DJ. Static and dynamic measures of frailty predicted decline in performancebased and self-reported physical functioning. J Clin Epidemiol. 2005;58(11):1188-98.

61. Bouman AIE. Home visiting program for older persons with poor health status [PhD thesis]. Maastricht: School for Public Health and Primary Care Maastricht University; 2008. 


\section{5}

A nurse-led interdisciplinary primary care approach to prevent disability among community-dwelling frail older people: a large-scale process evaluation

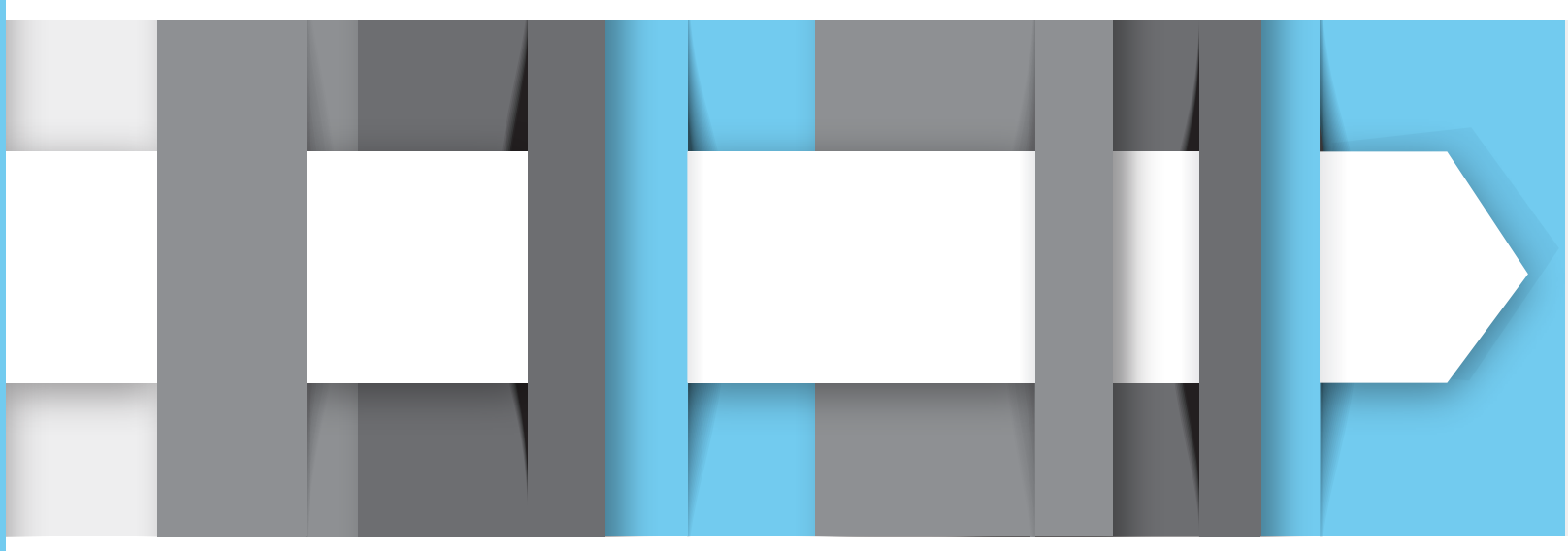

This chapter was published as: 


\section{Abstract}

Background: The complex healthcare needs of frail older people and their increased risk of disability require an integrated and proactive approach. In the Netherlands, an interdisciplinary primary care approach has recently been developed, involving individualised assessment and interventions (tailor-made care), case management and long-term follow-up. The practice nurse as part of a general practice is case manager and plans, organises and monitors the care process and facilitates cooperation between professionals. The approach has shown positive indications regarding its feasibility in a small pilot, but its implementation on a large scale had not hitherto been investigated.

Objectives: To examine the extent to which the interdisciplinary care approach is implemented as planned and to gain insight into healthcare professionals' and frail older people's experiences regarding the benefits, burden, stimulating factors and barriers.

Design: A process evaluation was conducted using a mixed methods design.

Settings: Six GP practices in the south of the Netherlands.

Participants: Practice nurses ( $n=7)$, GPs $(n=12)$, occupational therapists $(n=6)$ and physical therapists $(n=20)$ participated in the process evaluation. Furthermore, 194 community-dwelling frail older people ( $\geq 70$ years) were included using the Groningen Frailty Indicator. People who were terminally ill, were confined to bed, had severe cognitive or psychological impairments or were unable to communicate in Dutch were excluded.

Methods: Quantitative data (logbooks and evaluation forms) were collected from all the participating frail older people and thirteen semi-structured interviews with a selection of them were conducted. In addition, data from healthcare professionals were collected through twelve semi-structured interviews and four focus group discussions. Results: Although some parts of the protocol were insufficiently executed, healthcare professionals and frail older people were satisfied with the care approach, as provided a useful structure for the delivery of geriatric primary care and increased the attention to preventive treatment. Frail older people felt acknowledged by healthcare professionals and experienced support in handling their problems and fulfilling their wishes.

Conclusions: The findings of the study revealed several positive aspects of the interdisciplinary primary care approach. Given its complexity, the implementation of the nurse-led interdisciplinary care approach is challenging and some parts of the protocol need special attention.

\section{Background}

Frail older people often suffer from a combination of acute and chronic diseases (multimorbidity) and functional impairments leading towards disability and dependency on long-term care. ${ }^{1}$ In general, older people prefer to stay at home for as long as possible. ${ }^{2}$ However, community-based care is challenging, due to the complex healthcare needs of frail older people, which have to be addressed by various healthcare professionals. ${ }^{3}$

Beswick and colleagues (2008) have shown in their meta-analysis that complex interventions like community-based geriatric assessment and follow-up programs have the potential to support older people to live independently, although there is no clear evidence yet that one specific format is better than another. ${ }^{4}$ In the Netherlands several recent studies on proactive home visits for vulnerable older people showed conflicting results. These visits did not turn out to be effective ${ }^{5,6}$, or showed only modest short-term effects ${ }^{7}$. Despite the strong emphasis on primary care in the Dutch healthcare system, it may be assumed that the implementation of complex interventions is hampered by a fragmented and reactive approach and a lack of intense collaboration between healthcare professionals. ${ }^{8}$ Fragmented care is also a problem in other western countries. For example in the US the PACE programs have been introduced in many states to unite the fragmented healthcare financing and delivery system. PACE programs are comprehensive community-based care models for frail older people, who are eligible for nursing home admission. All needed preventive primary, acute and long-term care services are delivered by an interdisciplinary team in the community care setting focusing on the individual needs of community-dwelling frail older people and their families. ${ }^{9.10}$ There is consensus in the Netherlands that a shift has to be made towards a more integrated and proactive approach to prevent disability in community-dwelling frail older people successfully. ${ }^{11}$

A narrative review by Daniëls and colleagues (2010) offers a comprehensive overview of interventions studies focusing on disability prevention in communitydwelling frail older people..$^{12}$ There is a large diversity in content, disciplines involved duration, intensity, and setting. The reported effectiveness of interventions is not consistent, but elements such as interdisciplinary cooperation, individualised assessment and interventions (tailor-made care), case management and long-term follow-up seem to be promising with regard to disability prevention. In addition, self-management support and stimulating engagement in meaningful activities were recommended by the authors. ${ }^{12}$ Consequently, these elements were combined in an interdisciplinary primary care approach for community-dwelling frail older people, called 'Prevention of Care' (PoC)

The PoC approach starts with a postal screening for frailty followed by an extended multifactorial assessment conducted by a practice nurse (and, if needed 
other professionals). Based on the assessment phase the team of involved professionals formulate a preliminary treatment plan. Consequently, the practice nurse discusses this plan with the frail older person and his/ her informal caregiver resulting in a final treatment plan. A flexible toolbox of interventions, organised around five topics such as 'enhancing meaningful activities' or 'stimulating health' is available to guide the treatment. The toolbox is described in more detail elsewhere. ${ }^{8}$ Finally, the treatment plan is coordinated, monitored and evaluated by the practice nurse, who acts as case manager.13 The strength of the PoC protocol is that all elements mentioned above were combined in one comprehensive and interdisciplinary approach. The protocol offers healthcare professionals a useful structure for healthcare delivery (consisting of six steps) and practical tools for interdisciplinary, client-centered care, case management, behavioral change and self-management support.

The implementation of the $\mathrm{PoC}$ approach in daily practice is challenging due to the combination of a number of elements, which are based on literature ${ }^{12}$, but are relatively new in geriatric primary care practice. A pilot study among 41 frail older people and 10 healthcare professionals has shown positive indications regarding its feasibility, but implementation on a large scale has not been investigated yet.13 This article presents a comprehensive process evaluation of the PoC approach on a larger scale, among 194 frail older people and 45 healthcare professionals. The aim of the process evaluation was to provide insight into the extent to which the interdisciplinary PoC approach is implemented as intended. ${ }^{14}$ Furthermore, healthcare professionals' and frail older people's experiences regarding benefits, burden stimulating factors and barriers were evaluated. More insight into these factors may increase the understanding of its implementation and may lead towards an improved interdisciplinary care approach for frail older people in daily practice..$^{15}$

\section{Methods}

Study design

Between February 2010 and December 2011 the PoC approach was evaluated in a large-scale process evaluation among six GP practices in the south of the Netherlands. Several process evaluation components from Baranowski and Stables (i.e. reach, dose delivered, fidelity, dose received (exposure and satisfaction), and barriers) were chosen as theoretical base for the process evaluation. ${ }^{15-17}$ Table 1 shows an overview of these components, their definition and the related research questions.
Table 1 Components of the process evaluation and related research questions

\begin{tabular}{|c|c|}
\hline Theoretical element & Research question(s) \\
\hline \multicolumn{2}{|l|}{ Reach } \\
\hline $\begin{array}{l}\text { Proportion of the intended target } \\
\text { population that participated in the } \\
\text { care approach }\end{array}$ & $\begin{array}{l}\text { - Does PoC reach the target group } \\
\text { (frail older people)? } \\
\text { - What were the numbers of and reasons } \\
\text { for refusals and drop-outs? }\end{array}$ \\
\hline \multicolumn{2}{|l|}{ Dose delivered } \\
\hline $\begin{array}{l}\text { 'Amount' of delivered care } \\
\text { approach }\end{array}$ & $\begin{array}{l}\text { - To what extent was PoC performed } \\
\text { according to protocol? }\end{array}$ \\
\hline \multicolumn{2}{|l|}{ Fidelity } \\
\hline $\begin{array}{l}\text { Extent to which care approach was } \\
\text { delivered in intended manner and } \\
\text { spirit }\end{array}$ & $\begin{array}{l}\text { - To what extent was PoC implemented } \\
\text { consistently with its core principles? }\end{array}$ \\
\hline \multicolumn{2}{|l|}{ Exposure (dose received) } \\
\hline $\begin{array}{l}\text { Extent of active engagement in and } \\
\text { receptiveness to the care approach }\end{array}$ & $\begin{array}{l}\text { - What was the frail older people's and } \\
\text { informal caregivers' ability to understand } \\
\text { and implement the principles of PoC? }\end{array}$ \\
\hline \multicolumn{2}{|l|}{ Satisfaction (dose received) } \\
\hline $\begin{array}{l}\text { Satisfaction of older people and } \\
\text { health care professionals with the } \\
\text { care approach }\end{array}$ & $\begin{array}{l}\text { - What were the experiences of healthcare } \\
\text { professionals and frail older people } \\
\text { regarding PoC in terms of benefits, } \\
\text { satisfaction and stimulating factors? }\end{array}$ \\
\hline \multicolumn{2}{|l|}{ Barriers } \\
\hline $\begin{array}{l}\text { The extent to which problems were } \\
\text { encountered while applying the } \\
\text { care approach }\end{array}$ & $\begin{array}{l}\text { - What were the experiences of healthcare } \\
\text { professionals and frail older people } \\
\text { regarding PoC in terms of barriers? }\end{array}$ \\
\hline
\end{tabular}

Setting and participants

Professionals

Six GP practices in the region of Sittard and its surroundings (in the south of the Netherlands), involving twelve GPs and seven practice nurses implemented the PoC approach and were invited to participate in the process evaluation. The GP practices had no current active and systematic policy for the detection and follow-up of fra older people. Furthermore, six occupational therapists and twenty physical therapists, working in the same region, participated. 
Frail older people

Frail older people have an increased risk of adverse health outcomes, such as falls, hospitalisation, disability, and death. ${ }^{18}$ The reserve capacity of frail older people is reduced to a critically low point. Consequently, even small disturbances can lead to a series of complications. ${ }^{19}$ Despite the growing interest for frailty, there is an on-going debate of its definition. In general, there are two perspectives on frailty. On the one hand, a physical perspective describing frailty as a clinical syndrome characterised by multiple physical characteristics such as weight loss, fatigue or slow motor performance. ${ }^{18}$ On the other hand, from a multifactorial perspective frailty is explained by an accumulation of physical, psychological and social characteristics (e.g. physical problems, mood, and cognition). ${ }^{20,21}$ With regard to disability prevention multifactorial approach to frailty seems to be more suitable. ${ }^{8}$ As a consequence, the PoC approach takes a multifactorial perspective on screening, assessment and treatment. The Groningen Frailty Indicator (GFI), a self-report instrument that measures frailty from a multifactorial perspective, is used to identify community-dwelling frail older people ( $\geq 70$ years). ${ }^{22}$ Those older people scoring five or higher (range 0-15) were assumed to be frail and were included for the study $(n=194) .^{23}$ People who were terminally ill, were confined to bed, had severe cognitive or psychological impairments or were unable to communicate in Dutch were excluded in advance, based on the advice of their GP.

\section{Ethical Approval}

The study protocol and procedures were approved by the Medical Ethics Committee of the University Hospital Maastricht/ Maastricht University (\#09-3-067). Written informed consent from all participating frail older people was obtained.

The interdisciplinary care approach 'Prevention of Care' (PoC)

The core team of the interdisciplinary care approach PoC consists of a practice nurse and a GP. In the Netherlands, GPs have the role of a gatekeeper, who coordinates access to specialised and hospital care. The profession of practice nurses was introduced in 2001 to reduce the workload of Dutch GPs. ${ }^{24}$ They often work, under supervision of the GP, on disease prevention, chronic care management, menta health services, frail elderly assessments, and care of families with young children. ${ }^{25}$ To fulfill the role of case manager adequately, practice nurses need at least a bachelo degree, sufficient clinical expertise, effective communication and problem-solving skills, and a broad knowledge of the healthcare system, including financing, regulations, and resources. ${ }^{26}$ Nurses are well suited to provide this role of case manager, as the nursing process is similar to the process of case management including tasks such assessment of client needs, planning of care, and on-going coordination, monitoring, and evaluation of delivered care. However, principally othe professionals, for example a physiotherapist, an occupational therapist or a socia worker may fulfill this role as well. ${ }^{27}$ However, nurses in primary care are often the linking pin between patient and specific healthcare professionals. ${ }^{28}$

Within the PoC approach the practice nurse and the GP cooperate closely with occupational and physical therapists. If needed, other inpatient and outpatien healthcare professionals, for example, a pharmacist or a geriatrician can be involved as well. The PoC approach focuses on existing problems in performing daily activities and on risk factors for developing disability, and consists of six steps (see Table 2). The core principles of the approach are interdisciplinary cooperation, tailor-made care, selfmanagement support, and a focus on meaningful activities. The interdisciplinary care approach has been described in more detail elsewhere. ${ }^{8}$ An adapted version of the PoC approach has been developed for hospitals to increase the cooperation between primary and hospital care. However, its evaluation is not a part of the current study.

\section{Interdisciplinary cooperation}

Frail older people often have complex healthcare needs, which have to be addressed by various healthcare professionals. ${ }^{3}$ To ensure integrated care, the PoC protocol encompasses tasks, decision criteria, and working procedures for the healthcare professionals involved. A common care philosophy, defined roles, open and clea communication, regular structural communication, shared decision-making and goa setting are important determinants to enhance team cooperation. ${ }^{29,30}$

Tailor-made care and self-management support

Self-management support is based on the $5 A^{\prime}$ ' Behaviour Change Mode ${ }^{131}$, which combines a client-centred approach, a model of behavioural change (Stages of Change mode ${ }^{32}$ ) and motivational interviewing techniques ${ }^{33}$. Motivational interviewing provides practical tools for professionals to support self-management, which has been shown to be effective in improving clinical outcomes. ${ }^{34,35}$ Use of the $5 A^{\prime}$ 's Behavioural Model implies that goals and strategies are individually determined and will depend strongly on the frail older people's (self-perceived) problems, motivation and capabilities. The self-management skills of older people will influence whether goals are focused on the client or more on (support of) the social and physical environment. ${ }^{8}$

Meaningful activities

As the PoC approach aims to support frail older people in continuing to do those activities they enjoy or need to do, meaningful activities have to be at the core of the approach. Healthcare professionals need to explore the concerns or problems of older people regarding the performance of meaningful activities, need to understand the older people's priorities, and need to use meaningful activities, where possible 
Table 2 Steps of the interdisciplinary care approach 'Prevention of Care' (PoC)

\begin{tabular}{|c|c|}
\hline Steps & Content \\
\hline $\begin{array}{l}\text { Step 1: } \\
\text { Frailty Screening }\end{array}$ & $\begin{array}{l}\text { A postal questionnaire, including the } 15 \text {-item Groningen Frailty } \\
\text { Indicator (GFI)22 is used to identify frail older people based on a } \\
\text { GFI score } \geq 5 \text {. }\end{array}$ \\
\hline $\begin{array}{l}\text { Step 2: } \\
\text { Assessment }\end{array}$ & $\begin{array}{l}\text { The practice nurse of the GP visits frail older people for a } \\
\text { multidimensional assessment in the presence of the main } \\
\text { informal caregiver (if available). The focus is on existing problems } \\
\text { in performing daily activities and on risk factors for developing } \\
\text { disability. After the home visit, the practice nurse and the GP discuss } \\
\text { whether additional assessment by other inpatient or outpatient } \\
\text { healthcare professionals is needed. }\end{array}$ \\
\hline $\begin{array}{l}\text { Step 3: } \\
\text { Analysis and } \\
\text { preliminary } \\
\text { treatment plan }\end{array}$ & $\begin{array}{l}\text { Based on the assessment phase, a preliminary treatment plan is } \\
\text { formulated, whether in a bilateral meeting (practice nurse and GP) } \\
\text { or in an extended team meeting consisting of practice nurse, GP, } \\
\text { occupational and physiotherapist and, if necessary, other healthcare } \\
\text { professionals. The treatment plan includes goals, strategies and } \\
\text { responsibilities. }\end{array}$ \\
\hline $\begin{array}{l}\text { Step 4: } \\
\text { Agreement on } \\
\text { treatment plan }\end{array}$ & $\begin{array}{l}\text { During a second home visit, conducted by the practice nurse, a final } \\
\text { treatment plan is formulated together with the frail older person and, } \\
\text { if available, the informal caregiver. The plan involves goals, strategies } \\
\text { and responsibilities that fit their needs. The practice nurse involves } \\
\text { them in decision-making and establishes a cooperation, in which a } \\
\text { learning process begins, leading to new insights and possibly to new } \\
\text { goals and actions. }\end{array}$ \\
\hline $\begin{array}{l}\text { Step 5: } \\
\text { Executing the } \\
\text { treatment plan }\end{array}$ & $\begin{array}{l}\text { The intervention protocol offers recommendations and guidelines } \\
\text { for the execution of the treatment plan. For example, a flexible } \\
\text { toolbox of interventions is available which focuses on five topics: } \\
\text { - Meaningful activities } \\
\text { - Adapting the environment, activities or skills } \\
\text { - Social network and social activities } \\
\text { - Daily physical activity } \\
\text { - Stimulating health }\end{array}$ \\
\hline $\begin{array}{l}\text { Step 6: } \\
\text { Evaluation and } \\
\text { follow-up }\end{array}$ & $\begin{array}{l}\text { During and after the treatment the practice nurse evaluates with } \\
\text { the frail older person (and, if available, the informal caregiver) the } \\
\text { achievement of goals, the implementation of strategies in daily life } \\
\text { and the need for support in the following period. The professionals } \\
\text { involved will be updated about the agreements. }\end{array}$ \\
\hline
\end{tabular}

Examples of meaningful activities are gardening, visiting family/ friends, reading a book, taking a walk, playing games or joining religious activities. The experience of 'doing' may increase insight and beliefs in one's own capabilities (self-efficacy) ${ }^{36}$ which is central to self-management. Graff and colleagues (2006) showed the effectiveness of a focus on meaningful activities in a program for older people with dementia. $^{37}$

Training healthcare professionals

The healthcare professionals involved (practice nurses, GPs, occupational therapists and physical therapists) were trained for a 3-month period before the implementation of the PoC approach. They attended several meetings about the steps and core principles of the intervention, such as interdisciplinary cooperation (e.g. team meetings), tailor-made care, self-management support, meaningful activities and toolbox parts These meetings were more general and were held for all healthcare professionals. Discipline-specific meetings were also organised regarding the conduct of assessments and treatment by the different healthcare professionals. In addition, a meeting about geriatric syndromes (e.g. incontinence, malnutrition, falls) was organised.

During the implementation period, healthcare professionals had the opportunity to ask for supervision by the project group members (training on the job), if they experienced difficulties in implementing the $\mathrm{PoC}$ protocol. If healthcare professionals took little initiative towards support, members of the project group approached them more directly offering supervision. Project staff gave feedback and advice with regard to the new working methods and procedures and how to implement them in thei daily routines. One issue, for example, was how to organise a team meeting. In addition, ten lunch meetings for practice nurses and two evaluation meetings with al the healthcare professionals involved took place to exchange information and experiences.

\section{Data collection}

Quantitative and qualitative data collection methods were applied in a mixed methods design. The focus of the quantitative data collection (logbooks and evaluation forms) was on reach, dose delivered and frail older people's exposure to the PoC approach. Qualitative data methods (semi-structured and focus group interviews) were used to investigate the fidelity of the PoC approach and healthcare professionals' and frail older people's experiences with the PoC approach. Insight into their experiences is useful to explain the extent of reach, exposure and delivery of the care approach (dose delivered and fidelity). A combination of methods is useful to broaden the scope of the data (triangulation). ${ }^{38}$

Logbooks and evaluation forms

During the evaluation period, practice nurses were asked to fill in logbooks and evaluation forms for all the participating frail older people. The logbooks contained information about the 'amount' of care delivery (dose delivered), for example, the number of team meetings or referrals to other disciplines. The numbers of and 
reasons for refusals and drop-outs were registered here as well (reach). In the evaluation forms, practice nurses were asked to judge on an individual level the frailty status of the participating older people (reach) and their ability to understand the goal and the working method of the care approach and their adherence to commitments (exposure). ${ }^{15-17}$

Semi-structured and focus group interviews

Semi-structured and focus group interviews were used to assess the fidelity of the interdisciplinary care approach. Fidelity refers to whether the care approach was carried out in the intended manner and spirit (interdisciplinary cooperation, tailor-made care self-management support, and meaningful activities). Furthermore the experiences of healthcare professionals and frail older people in terms of benefits, burden, stimulating factors and barriers were investigated and recommendations regarding future implementation of the approach were collected. ${ }^{15-17}$

The semi-structured interviews with the practice nurses and the GPs were conducted at practice-level at two moments, half-way through the evaluation period (December 2010) and near the end (September 2011). A list of quality indicators, including the steps and key elements of the intervention, was used as a guideline for these interviews. At the end of the evaluation period (from October until December 2011), four focus group interviews with healthcare professionals were conducted. They were organized separately for GPs, practice nurses, and allied healthcare professionals (occupational therapists and physical therapists). The semi-structured interviews with frail older people $(n=13)$ were conducted half-way through the evaluation period (May and June 2011). Each practice nurse was asked to select several older people based on the following criteria: ability to do an interview (e.g sufficient cognition and hearing capacity), recent contact with the practice nurse (< two months ago), received at least one part of the toolbox (see Table 2), seen by at least one other discipline than the practice nurse. A self-developed and pre-tested topic list was used as a guideline for the interviews. Table 3 shows an overview of how the components of the process evaluation were operationalised and measured.

Data analysis

For the analysis of quantitative data, descriptive statistics (frequencies, means, and percentages) were calculated using software package SPSS for Windows, version 17.0. Qualitative methods were used for research questions that quantitative research cannot deal with. The focus group interviews and semi-structured interviews with older people were audio-taped and transcribed verbatim. To analyse qualitative data an integrated approach developed by Bradley and colleagues (1964) was applied. ${ }^{30}$ This approach combines the principles of inductive reasoning using predetermined code types. A coding list, based on the theoretical components derived from

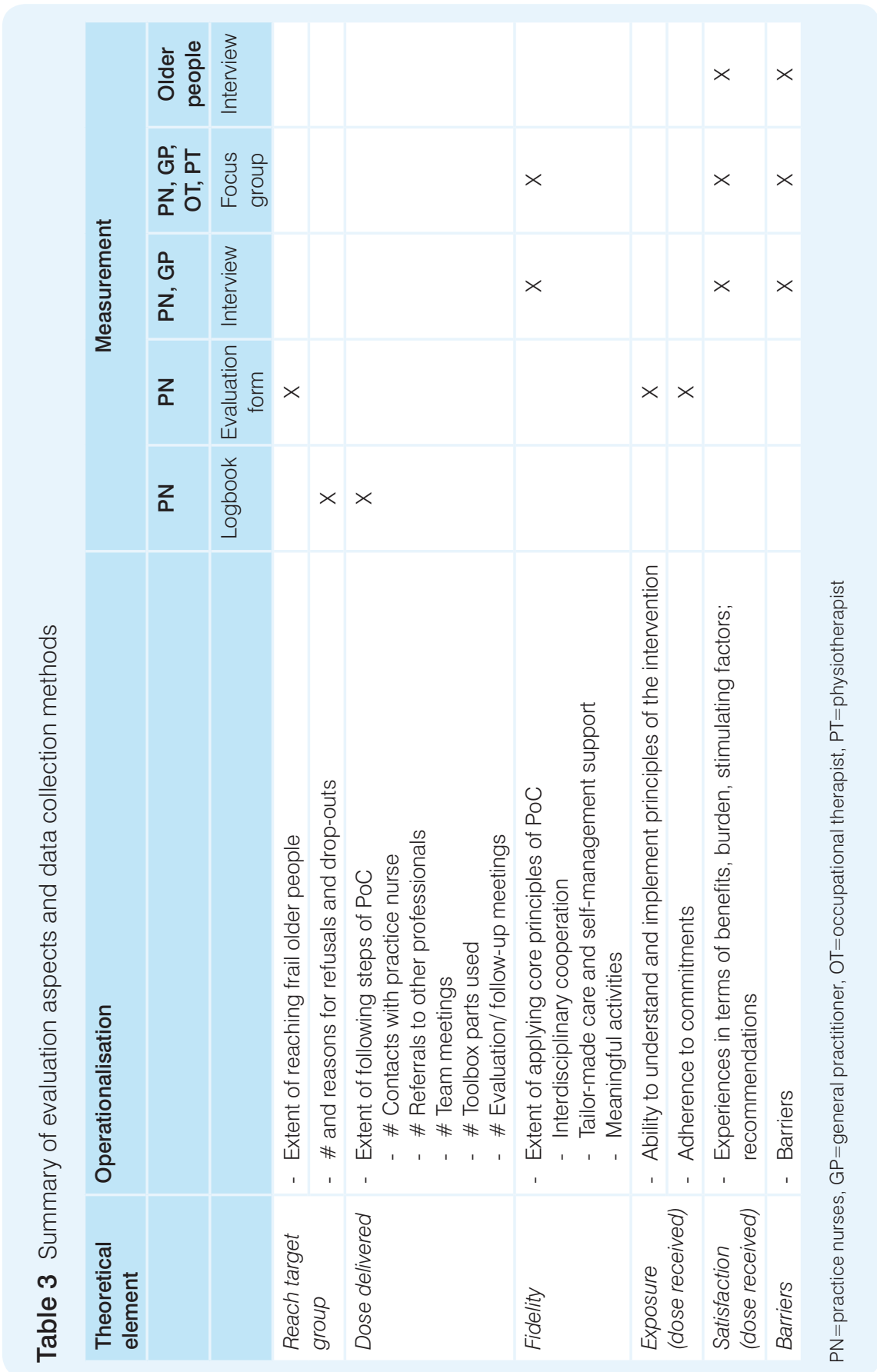


Baranowski and Stables (2000), was used to code the collected data. ${ }^{16}$ While all participating GPs and practice nurses were visited for a semi-structured interview, the amount of interviews with frail older people was established by applying the principal of theoretical saturation. This means that data saturation is reached, when no new insights are obtained, no new themes are identified, and no issues arise regarding a category of data. ${ }^{40}$ Saturation of all categories signifies the point at which to end the research. ${ }^{41}$ Through the coding process and constant comparison, theoretical saturation occurred after thirteen interviews. This choice can be considered as legitimate as there is some evidence in the literature that saturation often occurs within the first twelve interviews. ${ }^{42}$ The use of both, semi-structured and focus group interviews, increased the richness and trustworthiness of qualitative data. Researcher triangulation was used to increase the credibility and validity of the results. ${ }^{43}$ First, data were analysed independently by three members of the research group followed by a collaborative discussion about the data. Secondly, quantitative and qualitative data was integrated to confirm and cross-validate findings and resulted in an overal interpretation of the results (methods triangulation). The model of Baranowski and Stables (2000) was used as a structure to present the results. ${ }^{16}$

\section{Results}

Reach

On behalf of the six GP practices, 1,825 screening lists were sent to community-dwelling frail older people ( $\geq 70$ years) (see Figure 1). The response rate was $81 \%(n=1,477)$. According to the (GFI score $\geq 5)$ the prevalence of frailty was $33 \%(n=444)$. Nearly hal of the frail older people identified $(44 \%, n=194)$ were interested in participation and being included in the study. Of these, 45\% ( $n=87)$ were male and 46\% ( $n=90)$ lived alone (versus living together, $n=104)$. Most people $(60 \%, n=117)$ had no or lower than average education $(29 \%, n=56)$ or higher education $(11 \%, n=21)$. The mean age of the sample was 77.7 years $(s d=5.2)$ and the mean GFI score was $7.1(s d=1.9)$.

After a multifactorial assessment, conducted by the practice nurse during a home visit, practice nurses and GPs concluded that 36 older people (19\%) had no indication for/ no interest in care. These older people stopped with the PoC approach and received only the usual care if needed. They were significantly younger $(p=0.02)$ than the rest of the sample (on average 75.9 vs. 78.2 , respectively) and scored significantly better $(p=0.00)$ on the GFI (on average 6.3 vs. 7.3 , respectively). Furthermore, 29 out of 194 older people (15\%) stopped earlier with the PoC approach, due to health-related problems such as a decline in health or long-term admission $(n=13)$, a change-over to another GP practice $(n=12)$ or for other reasons $(n=4)$. They received usual care as well. These people were slightly older $(p=0.56)$
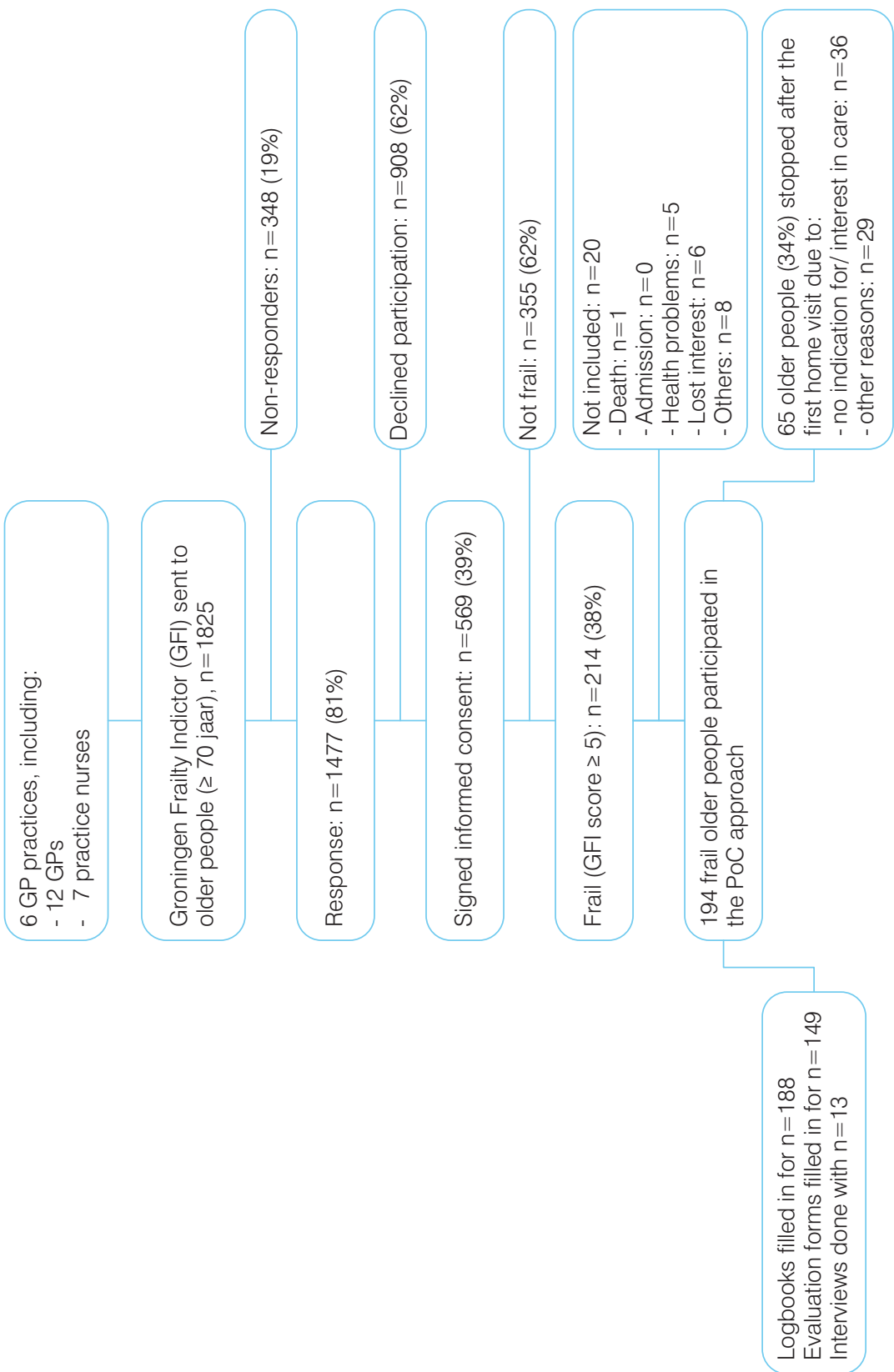
(on average 78.6 vs. 77.6 , respectively) and significantly frailer ( $p=0.05)$ (on average 7.7 vs. 7.0, respectively) than the rest of the sample.

The practice nurses filled in evaluation forms for 166 older people. For the analysis only complete evaluation forms were taken into account $(n=149)$. According to the practice nurses, 108 out of 149 older people $(73 \%)$ were frail and the PoC approach was appropriate for $69 \%$ of the older people identified $(n=102)$.

\section{Dose delivered}

Practice nurses filled in the logbook for 188 frail older people (six logbooks were missing). All frail older people received an extended assessment conducted by the practice nurse during a home visit, except for two individuals who died before the assessment could be arranged. Out of the 188 people, 82 (44\%) were seen by thei GP during the assessment phase. Referrals to an occupational therapist $(n=36)$, a physiotherapist $(n=28)$ or other disciplines ( $n=17)$ such as nutritionist, pharmacist, speech therapist or geriatrician were less frequent. The amount of referrals ranged from zero $(n=88)$ to four $(n=3)$. Most of the older people $(n=64)$ were referred to one other discipline. The preliminary treatment plan was formulated by the practice nurse and GP $(n=121)$ in an extended team meeting $(n=42)$ or by the practice nurse alone $(n=23)$. Some frail older people were discussed in a later phase in a bilateral meeting between the practice nurse and GP $(n=9)$ and/ or in an extended team meeting in which other disciplines were involved as well $(n=23)$. In $52 \%$ of the cases, the practice nurse formulated a plan together with the frail older person (and if available the informal caregiver) during a second home visit. The use of the toolbox parts ranged from $14 \%$ (Social network \& social activities) to $26 \%$ (Adapting environment, activities and skills). The number of toolbox parts received ranged from zero $(n=89)$ to four $(n=5)$. Some older people received one $(n=38)$ or two $(n=39)$ toolbox parts. Appointments for evaluation and follow-up were often (50\%) made between practice nurses and frail older people. In addition, in some cases evaluation and follow-up was discussed between healthcare professionals (in a bilateral or team meeting), but this occurred less often. Some older people were seen for evaluation and follow-up by the practice nurse, but were also discussed in a bilateral and/ or in an extended team meeting. Table 4 presents the extent to which the PoC protocol was delivered (dose delivered).

Fidelity

Interdisciplinary cooperation

One dominant topic during the semi-structured and focus group interviews was the substantial improvement of interdisciplinary cooperation. Before the implementation of the PoC approach, there was only occasionally contact between disciplines, mainly by telephone. According to all the healthcare professionals interviewed the
Table 4 Dose delivered of interdisciplinary care approach 'Prevention of Care' $(n=188)$

\begin{tabular}{|c|c|c|}
\hline Steps of Prevention of Care & $n(\%)$ & \\
\hline \multicolumn{3}{|l|}{ Screening } \\
\hline Send questionnaires & \multicolumn{2}{|l|}{1,825} \\
\hline Response & 1,477 & $81 \%$ \\
\hline Signed informed consent & 569 & $39 \%$ \\
\hline Frail according to GFI ( $\geq 5)$ & 214 & $38 \%$ \\
\hline Participated in PoC approach & 194 & $91 \%$ \\
\hline \multicolumn{3}{|l|}{ Assessment } \\
\hline Practice nurse & 186 & $99 \%$ \\
\hline \multicolumn{3}{|l|}{ Additional assessment } \\
\hline GP & 82 & $44 \%$ \\
\hline Occupational therapist & 36 & $19 \%$ \\
\hline Physiotherapist & 28 & $15 \%$ \\
\hline Other disciplines & 17 & $9 \%$ \\
\hline \multicolumn{3}{|l|}{ Analysis and preliminary treatment plan } \\
\hline Practice nurse & 23 & $12 \%$ \\
\hline Bilateral meeting (practice nurse, GP) & 121 & $64 \%$ \\
\hline Extended team meeting (practice nurse, GP and others) & 42 & $22 \%$ \\
\hline \multicolumn{3}{|l|}{ Agreement on treatment plan } \\
\hline Practice nurse & 97 & $52 \%$ \\
\hline \multicolumn{3}{|l|}{ Executing the treatment plan } \\
\hline \multicolumn{3}{|l|}{ Use of toolbox parts } \\
\hline - Meaningful activities & 32 & $17 \%$ \\
\hline - Adapting the environment, activities or skills & 48 & $26 \%$ \\
\hline - Social network \& social activities & 27 & $14 \%$ \\
\hline - Daily physical activity & 36 & $19 \%$ \\
\hline - Stimulate health & 41 & $22 \%$ \\
\hline Other interventions & 27 & $14 \%$ \\
\hline \multicolumn{3}{|l|}{ Evaluation and follow-up } \\
\hline Practice nurse and frail older person & 94 & $50 \%$ \\
\hline Bilateral meeting (practice nurse, GP) & 35 & $19 \%$ \\
\hline Extended team meeting (practice nurse, GP and others) & 54 & $29 \%$ \\
\hline
\end{tabular}


frequency of contacts, by telephone, email and in person, increased substantially by applying the principles of the PoC approach. Furthermore, interdisciplinary team meetings took place to discuss the (preliminary) treatment plan. Whether meetings were executed according to protocol depended strongly on the chair of the meeting (usually the practice nurse, otherwise the GP). Due to the open communication in the team, all the healthcare professionals interviewed felt free to share their own expertise. Practice nurses and GPs reported that they learned particularly more about the expertise of occupational therapists resulting in a rise in referrals. In contrast, the physiotherapists involved seemed disappointed as they experienced less increase in referrals as expected.

The roles in the team were clearly defined according to the healthcare professionals interviewed. Overlapping tasks were minimised by formulating an integrated treatment plan, involving collaborative goals and specified tasks for each discipline. Sometimes, other healthcare professionals, such as a social worker, homecare provider or pharmacist, were also invited to the team meetings. The role of the practice nurse was considered important by all the healthcare professionals interviewed. The practice nurse was case manager and responsible for the coordination and organisation of care. Also, monitoring and follow-up of frail older people and their informal caregivers was one of the tasks of the practice nurse. Evaluation and follow-up occurred via home visits or telephone contacts.

Tailor-made care and self-management support

All healthcare professionals interviewed reported that the PoC approach encouraged frail older people to think about their concerns and problems, their motivation and capabilities and their wishes for the future. The information was used to develop a preliminary treatment plan, including individual goals, strategies and responsibilities. The preliminary treatment plan was supposed to be discussed by the practice nurse with the frail older person (and informal caregivers if available) during a second home visit. The aim was to involve them in decision-making and to establish a cooperation, in which a learning process begins, leading to new insights and possibly to new goals and actions. However, some of the practice nurses interviewed reported that the treatment plan was often not discussed with the frail older person and some of these home visits were replaced by telephone contacts, which does not conform to the protocol.

Some of the healthcare professionals interviewed mentioned that they were not aware of applying the 5A's Behaviour Change Mode ${ }^{31}$, but the semi-structured and focus group interviews indicated that healthcare professionals considered the frail older peoples' readiness to change in their counselling style, adapted information and advice to their individual needs and respected their decisions. Furthermore, the principles of Motivational Interviewing were applied, which is also part of the 5A's Behaviour Change Model.
Meaningful activities

During the semi-structured and focus group interviews all the practice nurses interviewed reported that the concerns or problems of frail older people regarding the performance of meaningful activities were assessed during the assessment phase. According to the healthcare professionals interviewed, an integrated treatment plan was developed to fit the individual needs and preferences of older people.

\section{Exposure}

Based on the complete evaluation forms ( $n=149)$ filled in by practice nurses, $76 \%$ $(n=113)$ of the older people included understood the goal and the procedure of the PoC approach. Their adherence regarding the commitments made with the practice nurse was assessed as (very) good for 72 older people (48\%), sufficient for 46 people (31\%) and poor for 20 people (13\%). For 11 people (7\%) this question was not applicable, as no commitments were made.

Satisfaction and barriers experienced by healthcare professionals Benefits

According to all the healthcare professionals interviewed, the PoC approach provided a useful structure for the delivery of geriatric primary care and increased the attention to preventive treatment of frail older people. One of the greatest benefits of the approach was the improvement of interdisciplinary cooperation. Sharing information during the team meetings led towards a better understanding of concerns, problems and wishes of frail older people. The information acquired was relevant for the delivery of tailor-made care. Healthcare professionals also learned much about each other's expertise, which led to more consultations of involved healthcare professionals and more frequent referrals (occupational therapy). The clearly defined roles and tasks led to more efficient healthcare delivery. The possibility of expanding the team with other healthcare professionals was also appreciated by those interviewed

Burden

Despite their usefulness, team meetings were experienced as time-consuming and sometimes difficult to organise and required good organisation. Before the PoC approach, healthcare professionals were used to act immediately after identifying problem. According to the PoC protocol, healthcare professionals had to finish the assessment phase first, resulting in a collaborative treatment plan. Before they could start the treatment, the frail older person had to agree on this plan. This change in the culture of healthcare delivery was initially difficult for healthcare professionals, but after a while they recognised the added value of formulating a collaborative treatment plan 
Stimulating factors

In general, the PoC protocol provided a useful structure and tools for integrated community-based care of frail older people. According to all the healthcare professionals, the implementation of the protocol depended strongly on the professional skills of the practice nurse. GPs and allied professionals mentioned that an empathic capacity of the practice nurse and good organisation and communication skills had positive effects on the implementation of the PoC approach, while a lack of education, experience and capacities was considered as a barrier. Practice nurses reported that frail older people expressed much gratitude for the attention they received, which gave them a feeling of work fulfilment.

Barriers and recommendations

During the semi-structured and focus group interviews, doubts about the screening method were expressed by all practice nurses and GPs interviewed. In their opinion, the screening method worked insufficiently in identifying the appropriate target group for the PoC approach. On the one hand, the health status of some older people was too poor to participate. On the other hand, some older people were considered to be not (yet) frail. Practice nurses and GPs recommended a higher cut-off score on the Groningen Frailty Indicator ${ }^{22}$ or at least a cut-off score that is less sensitive to psychosocial problems. In their opinion, frail older people with a low score on the Groningen Frailty Indicator (GFI=5) often suffer from psychosocial problems, but have no indication for care. Practice nurses and GPs also indicated during the interviews that some frail older people might have been missed by the chosen screenings approach due to non-response or the exclusion criteria. They thought that case-finding based on the judgement of the practice nurse and GP would enable the identification of a more appropriate target group.

Some parts of the PoC protocol were time-consuming (e.g. assessment, team meetings) or difficult to apply (e.g. toolbox). The toolbox provided healthcare professionals with guidelines for assessment and treatment organised by different topics, but they did not know how to put these guidelines into practice. Also, how to use the theoretical models such as the 5A's Behaviour Change Mode ${ }^{31}$ remained unclear to them. According to the healthcare professionals interviewed, barriers to implementing the protocol were due to an overload of information during the training activities and a lack of training on the job. They recommended more supervision and opportunities to exchange experiences with other healthcare professionals. Furthermore, a digitalisation of forms was recommended by practice nurses and GPs to avoid double registration and to facilitate an exchange of data with other disciplines.
Satisfaction and barriers experienced by frail older people

Benefits

Frail older people reported in the interviews that they greatly appreciate thei independence. They prefer to take their own decisions and to find solutions by themselves. These norms and values fit the self-management character of the PoC approach. Frail older people saw one of the greatest benefits of the PoC approach in becoming aware of their needs and (potential) problems. Healthcare professionals istened carefully and spent much attention to the topics introduced by the frail older person. Frail older people felt acknowledged by healthcare professionals and experienced support in fulfilling their needs and handling their problems. A good relationship with the healthcare professionals involved was perceived as a safety net with regard to future problems.

Burden

There were hardly any burden mentioned by frail older people, except for the intensity and complexity of the PoC approach. For a few older people, participation was too time-consuming. Others experienced difficulties in distinguishing the disciplines involved

Stimulating factors

In general, frail older people did not have many expectations regarding participation but felt that there was no harm in trying it. Some frail older people expected to find a solution for their problems. The fact that the invitation to participate was sent by their GP enhanced their willingness to participate. For most of them, the GP is still an important and respected person and his or her advice was followed most of the time. A few older people mentioned that they were no longer used to get so much attention from their GP and they appreciated their initiative very much.

Barriers and recommendations

Frail older people did not experience specific barriers to the PoC approach. However they made the general recommendation that older people want to be taken seriously by healthcare professionals. Furthermore, healthcare professionals should consider that older people often struggle with irreversible losses such as the death of a dear person, which may result in frustration, loss of motivation and neglect of advice egarding care in general. 


\section{Discussion}

The aim of the process evaluation was to provide insight into the extent to which the interdisciplinary care approach PoC is implemented as planned. Furthermore healthcare professionals' and frail older people's experiences regarding its feasibility were evaluated. From the perspective of the healthcare professionals, the PoC approach provided a useful structure for the delivery of geriatric primary care and increased the attention to preventive treatment. Frail older people were satisfied, as they felt acknowledged by healthcare professionals and experienced support in handling their problems and fulfilling their needs. While frail older people experienced hardly any burden or barriers regarding the PoC approach, healthcare professionals made several recommendations for its improvement.

Firstly, they experienced barriers regarding the screening approach and recommended either a higher cut-off score on the Groningen Frailty Indicator or a case-finding approach based on the clinical judgement of the practice nurse and the GP. According to the literature, clinical judgement in itself seems insufficient to identify frail older people. ${ }^{44}$ Frailty can be easily overlooked by GPs, who are used to focus on specific medical diseases, whereas frailty is multidimensional in nature and results from a complex interplay of physical, psychological, social, and environmental factors. In addition, it is not feasible given the large populations, which have to be screened. Therefore a two-step approach consisting of a short screening integrated in the daily practice of the GP as the first step, and a more complex assessment as the second is assumed to be most promising. ${ }^{44}$ Such a two-step approach is included in the PoC approach, but the screening method for frailty (step 1) needs some improvement regarding its specificity. ${ }^{45}$ Secondly, some parts of the PoC protocol were (initially) time-consuming or difficult to apply (e.g. interdisciplinary team meetings, toolbox, 5A's Behaviour Change Model). Healthcare professionals recommended more training on the job and opportunities to exchange experiences with each other. How to improve the training with regard to the implementation of the PoC approach in daily practice needs special attention.

The evaluation of the logbooks showed that some parts of the PoC protocol were insufficiently executed. Firstly, the problem analysis and the development of a preliminary treatment plan was often not done in a bilateral or an extended team meeting (step 3) and only half of the treatment plans were discussed with the fral older person (step 4). Secondly, the toolbox parts were not frequently used in the treatment of frail older people (step 5). Thirdly, the extent of evaluation and follow-up, especially among the healthcare professionals, was limited (step 6). With regard to behavioural change, discussion of the treatment plan in the interdisciplinary team and between healthcare professionals and frail older people (step 3 and 4 ) is essentia to reach collaborative agreement about treatment goals. Furthermore, regular follow-up and feedback is important for behavioural change. ${ }^{46}$ Continuous collaboration, from the assessment (step 2) until evaluation and follow-up (step 6), is also an importan element in patient-centred interdisciplinary care. It recognises and values the expertise and perspectives of a variety of different healthcare professionals and enables a partnership between the healthcare professionals involved and the patient in decisionmaking. ${ }^{29}$

It is well-known that a gap exists between research and the translation of findings into practice, especially in the field of preventive and behavioral change interventions. Glasgow and Emmons (2007) focus on four categories of barriers. ${ }^{47}$ These include characteristics of (a) the intervention, (b) the target settings, (c) the research or evaluation design, and (d) interactions among the first three categories. With regard o intervention characteristics (a) it may be assumed that PoC particularly required oo much time and expertise from healthcare professionals. Furthermore, some parts of the protocol may be not packaged or 'manualised' enough to provide the care according to the protocol principals. The primary care setting (b) may also have had an impact on the extent of implementing the PoC approach. For example the structures of a predominantly reactive healthcare system in the Netherlands ${ }^{48}$ may work against a proactive approach in community-dwelling frail older people. Barriers regarding the research design (c) may be that the target group was not adequately identified. As we discussed earlier, some older people were not (yet) frail. More research should be done into the complexity and context of an intervention to increase the success of implementation into daily practice. ${ }^{47}$

Interdisciplinary collaboration is one of the core principles of the PoC approach and the healthcare professionals reported improved collaboration, but the limited extent of discussing and evaluating the treatment plan and using the toolbox parts is not in line with this finding. This may be explained by the structure of the healthcare system, in which most decisions regarding care are taken by the GP or practice nurse. ${ }^{29}$ It seems questionable, whether always the right decisions were made with regard to the involvement of other healthcare professionals. When implementing interdisciplinary care, the cultural change of professionals' attitudes and organisationa structures need to be considered further. ${ }^{29}$ Furthermore, factors related to team structure (e.g. team size and composition, organisational support) or team processes (e.g. goal setting, regular team meetings) may work as barriers or facilitators. More attention should be paid to these factors to reach effective and efficient interdisciplinary collaboration. ${ }^{49}$

The semi-structured and focus group interviews with healthcare professionals and frail older people gave some indication that engagement in meaningful activities, another core principle of the PoC approach, is insufficiently applied in healthcare delivery. They reported that meaningful activities were part of the assessment and treatment, but most of the time they talked about existing problems in performing 
daily activities and risk factors for developing disability instead of enhancing meaningful activities. Although frail older people suffer from a combination of physical, social and psychological losses the focus of care should be on their capacity to maintain quality of life instead of eliminating specific diseases or complaints. ${ }^{50}$ Maybe healthcare professionals undervalue the importance of meaningful activities, because of their 'everyday' nature, and the assumption that the desire to engage in activity is an in-built physiological mechanism that drives and satisfies people to meet basic needs and develop potential. ${ }^{51}$ Furthermore, the term 'meaningful activities' is an inherently difficult construct to define and assess, although it is rather important for healthcare professionals for the development of interventions. ${ }^{52}$ In future, more attention has to be paid to the identification of and participation in meaningful activities, as they contribute strongly to the sense of purpose and fulfilment in life, with significant implications for health, well-being and aging successfully. ${ }^{53}$

This process evaluation has some limitations. Firstly, previous studies have shown that healthcare professionals have difficulties in reflecting adequately on their functioning, as they perceive themselves as performing better than they actually do, maybe due to a lack of awareness of their own behaviour or socially desirable answers. ${ }^{54,55}$ The current study also shows some conflicting results. Healthcare professionals involved were very positive about the implementation of the PoC approach during the semistructured and focus group interviews. However, the logbooks showed that not all the steps of the PoC approach were applied. Video- or audiotaping would have been useful to add to the data collection methods to provide more valid information about the actual performance of healthcare professionals. Secondly, frail older people participating in the semi-structured interviews were selected by practice nurses. These frail older people may not be representative, as there is a risk that only 'success cases' were selected for the interviews. Selection bias may also have played a role in the focus groups with the healthcare professionals, as their participation was no mandatory and possibly only the most motivated and satisfied professionals joined the group. However, this study has some strengths as well. One of these is the use of a theoretical framework ${ }^{15-17}$ for the design of the process evaluation and the data collection and analysis. Thirdly, the use of a mixed methods approach, combining quantitative and qualitative data collection methods, improves the quality of the study. Both methods have their strengths and limitations; consequently an integration of different methods provides better findings than either a quantitative or qualitative approach alone. ${ }^{43}$

The PoC approach is very complex in nature, as it combines a number of relatively new elements in geriatric primary care. The more complex an intervention is, the more difficult its implementation in clinical practice may be. Even if healthcare professionals are aware of the need to change and willing to do so, it is difficult to modify well-established patterns of care, especially if the clinical environment is not conducive to change. Barriers can arise at the level of the patient, the individual professional, the healthcare team, the healthcare organisation, or the wide environment. ${ }^{56}$ Consequently, more research into the implementation of the PoC approach is needed, especially with regard to client-centred interdisciplinary care, behavioural change and engagement in meaningful activities.

\section{Conclusion}

To prevent disability in community-dwelling frail older people, complex interventions conducted by an interdisciplinary primary care team are needed, involving individualised assessment, tailor-made interventions and long-term follow-up. With regard to integrated care, nurses are recommended as case managers to plan organise and monitor the care process and to facilitate cooperation between professionals. The $\mathrm{PoC}$ approach is appreciated by healthcare professionals and frail older people and provides a useful structure for the delivery of geriatric primary care. However, given its complexity, the implementation of the protocol needs specia attention, especially with regard to client-centred interdisciplinary care, behavioura change and engagement in meaningful activities.

Acknowledgements

The authors would like to thank participating older people and healthcare professionals, the members of the research group: Walther Sipers (Orbis Medical Centre) Lilo Crasborn (MCC Omnes), Simone Denis (MCC Omnes), Marlou Wolters (MCC Omnes), Jil Bindels (Maastricht University) and their research assistants: Floor Koomen, Ine Hesdahl and Astrid Dello. This research is funded by the Dutch National Care for the Elderly Programme by The Netherlands Organisation for Health Research and Development (ZonMw 311070301), The Hague, the Netherlands. 


\section{References}

1. Fried LP, Ferrucci L, Darer J, Williamson JD, Anderson G. Untangling the concepts of disability, frailty, and comorbidity: implications for improved targeting and care. Gerontol A Biol Sci Med Sci. 2004;59(3):255-63.

2. Henderson EJ, Caplan GA. Home sweet home? Comunity care for older people in Australia J Am Med Dir Assoc. 2008;9(2):88-94.

3. Markle-Reid M, Browne G. Conceptualizations of frailty in relation to older adults. J Adv Nurs. 2003:44(1):58-68

4. Beswick AD, Rees K, Dieppe P, Ayis S, Gooberman-Hill R, Horwood J, Ebrahim S. Complex interventions to improve physical function and maintain independent living in elderly people: a systematic review and mata-analysis. Lancet. 2008:371(9614):725-35.

5. Van Hout HP, Jansen AP, van Marwijk HW, Pronk M, Frijters DF, Nijpels G. Prevention of adverse health trajectories in a vulnerable elderly population through nurse home visits: a randomized controlled trial. $J$ Gerontol A Biol Sci Med Sci. 2010;65(7):734-42.

6. Bouman A, Van Rossum E. Ambergen T, Kempen G, Knipschild P. Effects of a home visiting program for older people with poor health status: a randomized, clinical trial in the Netherlands. J Am Geriatr Soc 2008;56(3):397-404.

7. Melis RJ, van Eijken MI, Teerenstra S, van Achterberg T, Parker SG, Borm GF, van de Lisdonk EH, Wensing M, Rikkert MG. A randomized study of a multidisciplinary program to intervene on geriatric syndromes in vulnerable older people who live at home (Dutch EASYcare Study). J Gerontol A Biol So Med Sci. 2008:63(3):283-90

8. Daniels R, van Rossum E, Metzelthin S, Sipers W, Habets H, Hobma S, van den Heuvel W, de Witte L. A disability prevention programme for community-dwelling frail older persons. Clin Rehabil. 2011;25(11):963-74.

. National PACE AsSOC

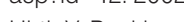

0. Hirth V, Baskins J, Dever-Bumba M. Program of all-inclusive care (PACE): past, present and future. J Am Med Dir Assoc. 2009;10(3):155-60

11. Nederlandse Huisartsen Genootschap \& Landelijke Huisartsen Vereniging. NHG/LHV-Standpunt: Het (ondersteunend) team in de huisartsenvoorziening. Utrecht: NHG/LHV;2011.

12. Daniels R, Metzelthin S, Van Rossum E, De Witte L, Van den Heuvel W. Interventions to prevent disability in frail community-dwelling older persons: an overview Eur J Ageing 2010;7(1):37-55

13. Daniels R. Frail Elderly - Identification and disability prevention in primary care [PhD Thesis]. Maastricht: School for Public Health and Primary Care, Maastricht University; 2011.

14. Harachi TW, Abbott RD, Catalano RF, Haggerty KP, Fleming CB. Opening the black box: using process evaluation measures to assess implementation and theory building. American Journal of Community Psychology. 1999;27(5):711-31.

15. Linnan L, Steckler AB. Process evaluation for public health interventions and research: an overview. Process evaluation for public health interventions and research. San Francisco. Jossey-Bass; 2002:1-23.

16. Baranowski T, Stables G. Process evaluations of the 5-a-day projects. Health Educ Behav. 2000;27(2):157-66

7. Saunders RP, Evans $\mathrm{MH}$, Joshi P. Developing a process-evaluation plan for assessing health promotion program implementation: a how-to guide. Health Promot Pract. 2005;6(2):134-47.

18. Fried LP, Tangen CM, Walston J, Newman AB, Hirsch C, Gottdiener J, Seeman T, Tracy R, Kop WJ, Burke G. McBurnie MA. Frailty in older adults: evidence for a phenotype. J Gerontol A Biol Sci Med Sci 2001;56(3):M146-56

19. Oude Voshaar RC, Benraad C, Olde Rikkert MGM. Kwetsbaarheid, complexiteit en welbevinden by ouderen. In: Leenties A, Gans ROB, Schols JMGA, van Weel C, eds. Handboek Multidisciplinaire Zorg Utrecht: De Tijdstroom; 2010:p. 43-62

20. Mitnitski AB, Graham JE, Mogilner AJ, Rookwood K. Frailty, fitness and late life mortality in relation to chronical and biological age. BMC Geriatr. 2002;2(1).

21. Rockwood K, Stadnyk K, MacKnight C, McDowell I, Herbert R, Hogan DB. A brief clinical instrument to classify frailty in elderly people. Lancet. 1999;353(9148):205-6.
22. Steverink N, Slaets JPJ, Schuurmans H, van Lis M. Measuring frailty: development and testing of the Groningen Frailty Indicator (GFI). Gerontologist. 2001;:41(1):236-7.

23. Metzelthin SF van Rossum E, de Witte LP. Hendriks MR, Kempen GI. The reduction of disability in community-dwelling frail older people; design of a two-arm cluster randomized controlled trial. BMC Public Health. 2010;10:511.

24. DerckX E. Eerste nurse practitioners met differentiatie huisartsenzorg. Tijdschr Verpleegkundigen 2006:3:26-30.

25. Van Weel C, Schers H, Timmermans A. Health Care in The Netherlands I Am Board Fam Med 2012;25(Suppl 1):S12-7.

26. White P, Hall ME. Mapping the literature of case management nursing. J Med Libr Assoc. 2006;94(2 Suppl):E99-E106.

27. Fraser KD, Strang V. Decision-making and nurse case management: a philosophical perspective. Adv Nurs Sci. 2004:27(1):32-43.

28. V\&VN Beroepsvereniging van Zorgprofessionals. Op een lijn komen. Visie op de rol van verpleegkundigen in de eerstelijnszorg. V\&VN. retrieved from http://www.venvn.nl2010

29. Orchard CA, Curran V, Kabene S. Creating a culture for interdisciplinary collaborative professional practice. Med Educ Online 2005;10

30. Jayadevappa R, Chhatre S. Patient centered care - a conceptual model and review of the state of the art Open Health Serv Policy J. 2011;4:15-25.

31. Glasgow RE, Emont S, Miller DC. Assessing delivery of the five 'As' for patient-centred counseling Health Promot Int. 2006;21(3):245-55.

32. Prochaska JO, DiClemente CC. Stages and processes of self-change in smoking: toward an integrative model of change. J Consult Clin Psychol. 1983;51(3):390-5

33. Miller WR, Rollnik S. Motivational Interviewing: preparing people for change. New York: Guildford Publications; 2002.

34. Bodenheimer T, Grumbach K. Improving primary care. Strategies and tools for better practice. New York Lange Medical Books/McGraw-Hill; 2007.

35. Chen SM. Creedy D, Lin H. Wollin J. Effects of motivational interviewing intervention on self-management psychological and glycemic outcomes in type 2 diabetes: a randomized controlled trial. Int I Nurs Stud 2012:49:637-44

36. Kielhofner G. Model of Human Occupation. Theory and application. Baltimore: Kluwer/Lippincot Williams\&Wilkins Wolters; 2008

37. Graff MJ, Vernooij-Dassen MJ, Thijssen M, Dekker J, Hoefnagels WH, Olde Rikkert MG. Community based occupational therapy for patients with dementia and their care givers: randomised controlled tria BMJ. 2006;333(7580):1196

38. Verbeek H, Zwakhalen SMG, van Rossum E, Kempen GIJM, Hamers JPH. Small-scale, homelike facilities in dementia care: a process evaluation into the experiences of family caregivers and nursing staff. Int J Nurs Stud. 2012;49:21-9.

39. Williamson J, Stokoe IH, Gray S. Old people at home: their unreported needs. Lancet. 1964;1:1117-20. . Strauss A, Corbin J. Basics for quallitative research: Grounded theory procedures and techniques. Newbury Park, CA: Sage Publications 1990.

41. Morse JM. The significance of saturation. Qual Health Res. 1995;5(2):147-9.

2. Guest G, Bunce A, Johnson L. How many interviews are enough? An experiment with data suturation and variability. Field Methods. 2006;18(1):59-82.

43. Boeije H. Analysis in Qualitative Research. London: SAGE Publications; 2010

44. De Lepeleire J, liffe S, Mann E, Degryse JM. Frailty: an emerging concept for general practice. Br J Gen Pract. 2009;59(562):177-82.

45. Daniels R, Van Rossum HIJ, Beurskens A, Van den Heuvel W, De Witte L. The predictive validity of three self-report screening instruments for identifying frail older people in the community. BMC Public Health 2012;12:69.

46. Bodenheimer T, Handley MA. Goal-setting for behavior change in primary care: an exploration and status Patient Educ Couns 2009;76:174-80. 
47. Glasgow RE, Emmons KM. How can we increase translation of research into practice? Types of evidence needed. Annu Rev Public Health. 2007;28:413-33.

48. European Commission. Synthesis report on the public consultation on the European Innovation Parthership on Active and Healthy Ageing retrieved from http://ec.europa.eu/researchlinnovation-union index_en.cfm?section=active-healthy-ageing\&pg=consultation.2011.

49. Xyrichis A, Lowton $\mathrm{K}$. What forsters or prevents interprofessional teamworking in primary and community care? A literature review. Int J Nurs Stud. 2008;45(1):140-53.

50. Nicholson C, Meyer J, Flatley M, Holman C. The experience of living at home with frailty in old age: a psychosocial qualitative study. Int J Nurs Stud. 2012;50(9):1172-9.

51. Harmer BJ, Orrell M. What is meaningful activity for people with dementia living in care homes? A comparison of the views of older people with dementia, staff and family carers Aging Ment Health 2008;12(5):548-58.

52. Eakman AM. Measurement characteristics of the Engagement in Meaningful Activities Survey in an
age-diverse sample Am J Occup Ther. 2012;66(2):20-9. 3. Eakman AM, Carlo ME, Clark FA The Mearingt Aciv

3. Eakman AM, Carlson ME, Clark FA. The Meaningful Activity Participation Assessment: a measure of
engagement in personally valued activities. Int J Aging Hum Devel. 2010;70(4):299-317. 54. Dorresteijn TAC, Zijlstra GAR, van Haastregt JCM, van Vlaeyen JWS, Kempen GIJM. Feasibility of a nurse-led in-home cognitive behavioral program to manage concerns about falls in frail older people: a process evaluation. Res Nurs Health. 2013;36(3):257-70.

55. Heinich E. Diabetes self-management strategles 1050 .

Thesis]. Maastricht: Health Medicine and Life Sciences, Maastricht University; 2012.
Grol R, Grimshaw J. From best evidence to best practice: effective implementation of change in patients

care Lancet. 2003;362(9391):1225-30. 


\section{6}

Effectiveness of an interdisciplinary primary care approach to reduce disability in community-dwelling frail older people: a cluster randomised controlled trial

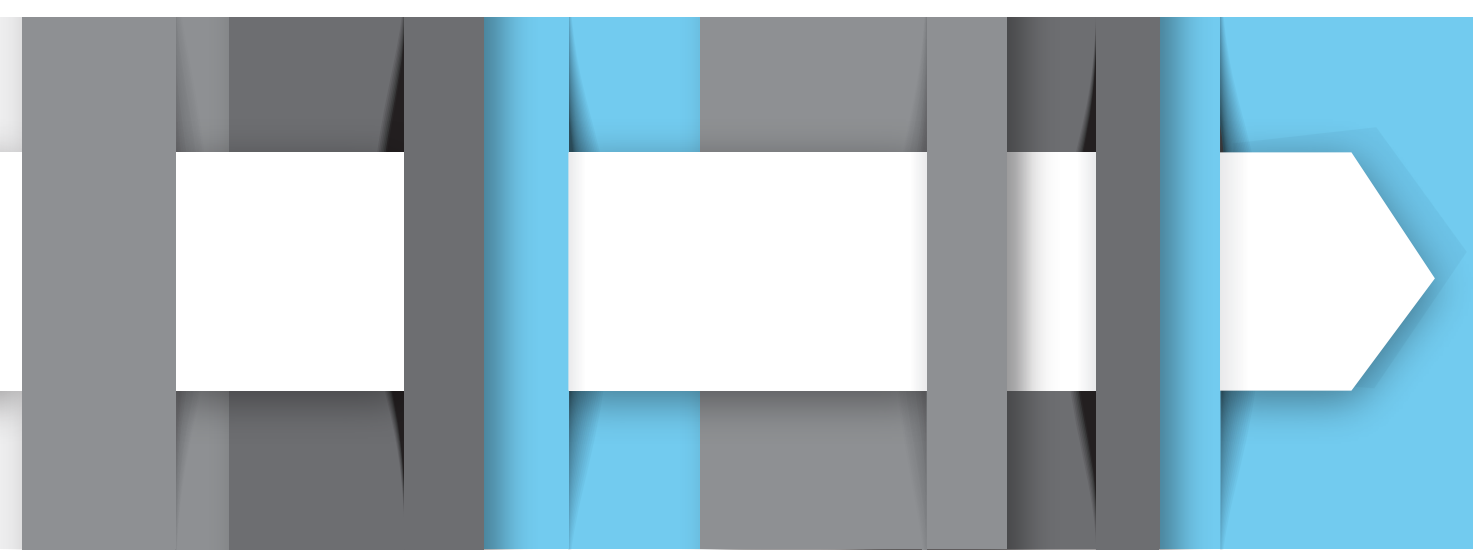

This chapter was published as: Metzelthin, SF, van Rossum, E, de Witte, LP, Ambergen, AW, Hobma, SO,

Sipers, W, Kempen, GlJM Bristish Medical Journal 2013;347:f5264 


\section{Abstract}

Objective: To evaluate whether an interdisciplinary primary care approach for community-dwelling frail older people is more effective than usual care in reducing disability and preventing (further) functional decline.

Design: Cluster randomised controlled trial.

Setting: Twelve general practices in the south of the Netherlands

Participants: 346 frail older people (score $\geq 5$ on Groningen Frailty Indicator) were included; 270 (78\%) completed the study.

Interventions: General practices were randomised to the intervention or contro group. Practices in the control group delivered care as usual. Practices in the intervention group implemented the 'Prevention of Care' (PoC) approach, in which frail older people received a multidimensional assessment and interdisciplinary care based on a tailor-made treatment plan and regular evaluation and follow-up.

Main outcome measures: The primary outcome was disability, assessed at 24 months by means of the Groningen Activity Restriction Scale. Secondary outcomes were depressive symptomatology, social support interactions, fear of falling, and social participation. Outcomes were measured at baseline and at 6, 12, and 24 months' follow-up.

Results: 193 older people in the intervention group (six practices) received the PoC approach; 153 older people in the control group (six practices) received care as usual. Follow-up rates for patients were $91 \%(n=316)$ at six months, $86 \%(n=298)$ at 12 months, and $78 \%(n=270)$ at 24 months. Mixed model multilevel analyses showed no significant differences between the two groups with regard to disability (primary outcome) and secondary outcomes. Pre-planned subgroup analyses confirmed these results.

Conclusions: This study found no evidence for the effectiveness of the PoC approach. The study contributes to the emerging body of evidence that communitybased care in frail older people is a challenging task. More research in this field is needed.

Trial registration: Current Controlled Trials ISRCTN31954692.

\section{Introduction}

In our ageing society the care for older people is one of the greatest challenges in healthcare.1,2 Evidence suggests that community-based care in comparison with institutionalisation may achieve better outcomes at lower costs and is also preferred by older people themselves. ${ }^{3,4}$ Consequently, an increasing demand exists for innovative initiatives to provide cost-effective community-based care. ${ }^{5,6}$ In most Western countries, such as the United Kingdom (UK) and the Netherlands, genera practitioners (GPs) have a central position in the provision of community care, as they are gatekeepers to specialised and hospital care. ${ }^{7}$ In the UK, GPs are required since 1990 to offer an annual multidimensional assessment to their patients aged 75 years and over. ${ }^{8}$ In addition, GPs geographical proximity to older people and their intense and long-lasting relationship with their patients may contribute to effective care in older people.? However, community-based care in frail older people is challenging. Frail older people have multiple and complex health care needs, which often lead to disability. ${ }^{9-11}$ Disability is defined as difficulty or dependency in the execution of daily activities that are essential for independent living. ${ }^{9}$ As disability is considered dynamic process, older people can recover to a less or non-disabled state. ${ }^{12}$ Regardless, preventive actions have to be taken to improve the abilities of frail older people to remain at home as long as possible. ${ }^{13}$

Daniëls and colleague ${ }^{14}$ did a narrative review to provide an extensive overview of existing interventions for prevention of disability in community-dwelling frail olde people. The identified interventions, most of which were in the field of comprehensive geriatric assessment and physical exercise programmes, showed a large diversity in terms of content, disciplines involved, duration, intensity and setting. Only a small number have shown beneficial effects with regard to disability, and most studies did not report on any long-term effects..$^{14}$ On the basis of this review, the authors suggested that community-based care interventions for frail older people should be conducted by an interdisciplinary primary care team involving individualised assessments and interventions (tailor-made care), self-management support, engagement in meaningful activities, case management and long-term follow-up. In an effort to reduce disability and preventing (further) functional decline in communitydwelling frail older people, in the present study these elements were combined into one approach: the 'Prevention of Care' (PoC) approach. This approach focuses on both older people with an increased risk for developing disability and older people who are already disabled. ${ }^{15}$ A previous pilot study $(n=41)$ using the PoC approach has shown promising results..$^{15}$ Older people appreciated the attention they got and felt supported in reaching their goals and in handling future disability. Healthcare professionals reported that the approach provided a useful structure for geriatric primary care. In addition, the approach stimulated interdisciplinary collaboration, a 
focus on meaningful activities and self-management support.15 However, the effectiveness with regard to disability and various related outcomes has not yet been studied. Consequently, we conducted this trial to investigate the effectiveness of the PoC approach on various patient level outcomes compared with usual care. ${ }^{16}$ We chose a cluster randomised design for practical reasons and to avoid contamination bias."

\section{Methods}

Study design

We did a two-arm cluster randomised controlled trial among twelve GP practices in the south of the Netherlands. Before the screening procedure for identifying frail older people started, we randomly allocated six practices to the PoC approach and six practices continued care as usual. Before randomisation, the GP practices were pre-stratified into four strata based on the number of older patients $(<350$ patients versus $\geq 350$ patients) and location (urban versus rural area). We assumed that GPs working in a practice with a large number of older patients have more experience with geriatric care and that older people living in a rural area receive more support from the informal care system than do those living in an urban area. We stratified the practices in pairs and used a computer generated randomisation list to randomise them into either intervention or control group. To promote extrapolation of the results, practices in an urban area with a large number of older people had twice the chance of being included in the study than practices in the other three strata. The cluster randomised controlled trial was performed as planned. More details of the study design have been published elsewhere. ${ }^{16}$

\section{Participants}

We invited all GP practices in the region of Sittard (the Netherlands) and its surrounding area that had no current active and systematic policy for the detection and follow-up of frail older people to take part in the study. In total, 24 GP practices were interested of which we randomly selected twelve for the study on the basis of a computer generated list of numbers. The study focused on their community-dwelling frail older patients ( $\geq 70$ years). Those who were terminally ill, were confined to bed, had severe cognitive or psychological impairments or were unable to communicate in Dutch were excluded based on the advice of the GP. The remaining other older people $(n=3,498)$ in the twelve practices received a postal questionnaire, including the Groningen Frailty Indicator (GFI). ${ }^{18}$ In the literature, a score of four or higher (range $0-15$ ) is proposed as the cut-off point for moderately to severely frail older people.1 However, this study focused on people who were considerably frail, ranging from people who have an increased risk for developing disability to disabled older people. Therefore, older people who signed the informed consent form and had a GFI score of five or higher were included in the study. For practical reasons, the recruitment of frail older people took place in three cycles. The first cycle started in December 2009 the second in February 2010 and the third in March 2010. The intervention and the collection of data also took place in three cycles. All included older people gave written informed consent prior to collection of the baseline measure. This study was approved by the Medical Ethical Committee of the Maastricht University/ Academic Hospital Maastricht in the Netherlands in 2009 (MEC 09-3-067)

\section{Intervention}

In the intervention group (six GP practices) frail older people received the PoC approach. The GP and practice nurse built the core team of the interdisciplinary care approach. In 2001 the profession of practice nurses was introduced in the Netherlands to reduce the workload of Dutch GPs who are the gatekeeper to specialised and hospital care. ${ }^{19}$ Practice nurses often work, under supervision of the GP, on disease prevention, chronic care management, mental health services, assessments among frail older people, and care of families with young children. ${ }^{20}$ Within the PoC approach the GP and his practice nurse cooperate closely with occupational and physiotherapists. If needed, other inpatient and outpatient healthcare professionals, for example, a pharmacist or a geriatrician are involved as well.

The PoC approach aims to reduce disability and prevent (further) functional decline using a six step approach (Figure 1). ${ }^{21}$ After the postal screening for frailty using the GFI (step 1), frail older people and their informal caregiver, if available, receive a home visit by the practice nurse who does a multidimensional assessment focusing on existing problems in performing daily activities and on risk factors for disability (step 2). The focus is on activities, which are meaningful to the older person. Examples of meaningful activities are gardening, visiting family/ friends, reading a book, taking a walk, playing games or joining religious activities. After the home visit, the GP and practice nurse discuss whether additional assessments by other inpatient or outpatient healthcare professionals are needed. On the basis of the assessment phase, a preliminary treatment plan is formulated (step 3), either in a bilateral meeting (GP and practice nurse) or in an extended team meeting consisting of a GP, practice nurse, occupational and physiotherapist and, if necessary, other healthcare professionals.

During a second home visit by the practice nurse (step 4), a final treatment plan is formulated, including a list of goals, strategies and actions that meet the olde person's needs. Depending on the self-management skills and preferences of the older person, strategies and actions are either focused on the older person or more on (support of) the social and physical environment. On the basis of the 5A's 
Step 1: Frailty screening

15 item Groningen Frailty Indicator (GFI); GFI score $>5$ :

practice nurse calls for home visit

\section{Step 2: Assessment}

Pratice nurse visits frail older people (and informal caregiver) for multidimensional assessment, which focuses on:

- Existing problems in performing daily activities and

- Risk factors for developing disability

Followed by decision making with general practitioner about need for

- Assessment by general practitioner

- Assessment by occupational therapist or physiotherapist (in case of concerns or (addition in pertorming activites)

\section{Step 3: Analysis and preliminary treatment plan}

- Bilateral meeting (glaneral practitioner and practice nessenent, whether in

se, occupational and

physiotherapist, and others)

\section{Step 4: Agreement on treatment plan}

Practice nurse discusses final treatment plan (involving goals, strategies, and second home visit

Step 5: Executing treatment plan
Intervention protocol offers recommendations and guidelines for execution of
treatment plan.
For example, a flexible toolbox of interventions is available focussing on five topics:
- Meaningful activities
- Adapting environment, activities, or skills
- Social network and social activities
- Daily physical activity
- Stimulating health
Practice nurse is case manager and monitors progress and satisfaction

Practice nurse is case manager and monitors progress and satisfaction

\section{Step 6: Evaluation and follow-up}

During and after treatment, practice nurse evaluates with frail older people

(and informal caregiver) achievement of goals, implementation of strategies in

be updated about agreements made

Figure 1 Six steps of the 'Prevention of Care' (PoC) approach.
Behavioural Change Model22 and motivational interviewing techniques ${ }^{23}$ the practice nurse encourages active involvement in decision-making and establishes cooperative working relationship with the frail older person and the informal caregiver. Subsequently, the treatment starts (step 5). The intervention protocol offers recommendations and guidelines for the execution of the treatment plan. For example, a toolbox of interventions is available that focuses on five topics: 'enhancing meaningful activities', 'daily physical activity', 'social network and social activities', 'adapting the environment, activities or skills' and 'stimulating health'. The practice nurse is also the case manage and, along with the frail older person and the informal caregiver, regularly evaluates the achievement of goals, the implementation of strategies in daily life and the need for support in the following period (step 6). The professionals involved are updated about the progress and the agreements made. The remaining six practices (contro

\section{Case summary}

AK is a 75 year old woman living independently in a small flat. She has four children, who live in the same city. Her husband has been living in a nursing home for two years.

AK received a letter from her general practitioner, who asked her to fill in the Groningen Frailty Indicator (step 1). She had a total frailty score of 7 , and the practice nurse called her to offer a home visit for a multidimensional assessment (step 2). The assessment focused on existing problems in performing daily activities and risk factors for developing disability. The practice nurse also discussed with AK her individual needs and goals and her motivation to make changes in her life. AK's most important goal was to stay independently in her home. She experienced problems with cooking, shopping, and visiting her husband in the nursing home. The last of these was particularly meaningful to her. She often felt exhausted and suffered from fear of falling. In addition, memory deficits affected her participation in daily life. Her chronic diseases (diabetes and heart failure) were under control.

After the home visit, the practice nurse discussed the results of the assessment with the general practitioner and decided to refer AK to a geriatrician for further cognitive assessment. In addition, they agreed that involving an occupational therapist and physiotherapist would also be useful, as AK had problems with daily activities. An interdisciplinary team meeting, consisting of the general practitioner, practice nurse, occupational therapist, and physiotherapist took place to formulate a preliminary treatment plan based on the results of the assessment (step 3). The assessment of the geriatrician showed no signs of dementia. Regarding her fear of falling, the team assumed that AK needed 
to change her attitudes and self-efficacy beliefs with regard to falling, leading towards improved participation in daily activities such as shopping and visiting her husband. An increase in physical activity was supposed to positively affect her fear of falling as well. In addition, simple strategies and a few helping aids were discussed to help AK with cooking and handling her memory deficits.

After the team meeting, the practice nurse visited AK again to finalise the treatment plan (step 4). Which toolbox parts could be used was also discussed. For the treatment of AK the toolboxes "adapting the environment, activities, or skills" and "daily physical activity" were chosen (step 5). During the treatment, the practice nurse visited AK four times to evaluate the achievement of goals and the implementation of strategies in daily life (step 6). Four months later during the last visit, AK reported that she had fewer problems with cooking and visiting her husband. She had increased her physical activity in daily life and had less fear of falling. However, the strategies learnt for handling her memory deficits were still difficult to apply in daily life. A few helping aids and a stoo placed in the kitchen helped her to cook more efficiently. The practice nurse will visit AK every six months to follow-up with her.

group) continued to deliver care as usual. The box illustrates how the approach works.

Measurements

We measured data for the effectiveness analysis at the level of the patient at baseline and after 6,12 and 24 months by using postal questionnaires and telephone interviews. Whereas older people and healthcare professionals were aware of the allocated arm (intervention or control), outcome assessors were kept blinded to the allocation.

\section{Outcome measures}

We measured the primary outcome, disability, at 24 months by means of the Groningen Activity Restriction Scale (GARS). ${ }^{24}$ This is an easy to administer comprehensive, reliable, hierarchical and valid measure for assessing disability in older people. It consists of two subscales. The first subscale is about activities of daily living (ADL) (eleven items), and the second subscale relates to instrumenta activities of daily living (IADL) (seven items). The scores for the total scale range from 18 to 72 with higher scores indicating more disability. ${ }^{24}$ As disability is strongly related to psychological and social functioning, ${ }^{25}$ we chose depressive symptomatology (depression subscale Hospital Anxiety and Depression Scale), ${ }^{26}$ social support interactions (Social Support List-Interaction version), ${ }^{27}$ fear of falling (Short Falls Efficacy Scale-International) ${ }^{28}$ and social participation (Maastricht Social Participation Profile, subscale A) ${ }^{29}$ as secondary outcomes. In addition, we used the Pearlin Mastery Scale to determine the feelings of competence and control in older people, ${ }^{30}$ feelings crucial for self-management and coping, ${ }^{31}$ which belong to the importan underlying mechanisms of the PoC approach.

Statistical analysis

We used descriptive techniques to describe the study groups. We compared baseline variables to detect differences between the intervention and control group at the start of the study. Because of the cluster randomised design of the study including three evels (GP practices, participants and repeated measurements), we applied a mixed model multi-level analysis. We analysed the primary and secondary outcomes, measured on the level of the patient, according to the intention to treat principle. We imputed missing values on the level of the scale by means of multiple imputations We based the maximum number of missing values within a scale on the guidelines given by the developers. If no guidelines were available, we accepted a limit of $25 \%$ missing values. Multi-level analyses are quite robust against missing values on measurement level. Therefore, we needed at least the baseline measurement and one out of three follow-up measurements to include older people in the analyses. For all analyses, we used a standard model including six independent variables. We corrected outcome estimates of the multilevel analyses for age, sex, educationa evel, significant differences at baseline (frailty and disability) and the baseline status of the outcome variable (in case of secondary outcomes) by including these variables as covariates in each model. We obtained insight into the effectiveness of the PoC approach in comparison with usual care at various follow-up times by examining fixed effects for group by time interaction. We evaluated the trend in time by removing the interaction term (group by time) from the model and testing only fixed effects for group and time. In a few imputed data sets, variance of GP practice iterated to zero. Consequently, we have examined in a basic model of disability, including only baseline status of disability as a covariate, whether GP practices had an impact on outcomes. The analyses of the basic model with and without GP practice as random effect have shown that results were highly similar for the two analyses. Therefore, we decided to exclude GP practice as an extra level.

We did several subgroup analyses. Firstly, we divided older people in the intervention group into two subgroups on the basis of their exposure to the PoC approach. We compared older people who received only assessment(s) (exposure group low) with those who received interventions, follow-up visits, or both (exposure group high). We tested fixed effects for exposure group by time interactions for significance. In addition, we did pre-planned subgroup analyses for the potential 
effect modifiers baseline status of frailty and mastery.16 We created two groups for each effect modifier based on the median scores: low frailty (GFI score 5-6) versus high frailty (score 7-14) and low mastery (PMS score 23-32) versus high mastery (score 10-22). Again we tested fixed effects for effect modifier by group interactions for significance.

The sample size calculation was based on our primary outcome (disability). On the basis of a power of $80 \%$ and an alpha of 0.05 (two-tailed testing), and an expected treatment difference of at least 2.0 points on the GARS, ${ }^{24}$ the required sample size was $n=80$ per group ( $N=160$ in total). Accounting for a drop-out rate of $30 \%$ and a cluster effect of $1.73(\mathrm{ICC}=0.05),{ }^{16}$ assuming equal cluster sizes, the final sample size had to be 180 per group (360 in total). ${ }^{16}$ We used software package SPSS for Windows, version 20.0, for all statistical analyses.

\section{Results}

We allocated twelve GP practices at random to the control (six practices) or intervention group (six practices). Half of the practices had less than 350 patients and half of them had at least 350 patients. In addition, six practices were located in an urban area and six in a rural area. These cluster characteristics were equally distributed among the groups. As shown in Figure 2, 3,498 community-dwelling older patients ( $\geq 70$ years) of the twelve practices received the screening questionnaire. The response rate was $80 \%(n=2,790)$. Non-responders were significantly younger than responders (mean age 76.75 years vs. 77.62 years, $\mathrm{p}<0.05$ ) and slightly non-responders were male $(42.9 \%$ vs. $39.1 \%, p=0.07)$. Older people who completed the questionnaire and were willing to participate in the study $(n=1,101)$ were significantly frailer than respondents who completed the questionnaire but declined participation $(n=1,634)$ (mean score on GFI 3.64 vs. 2.96, $p<0.05$ ). Severa participants declined participation and the questionnaire was not (completely) filled in $(n=55)$, thus, we were not able to obtain GFI scores and have no information about the level of frailty. Of the older people who were willing to participate, $34 \%(n=179)$ in the control group and $38 \%(n=214)$ in the intervention group were frail according to their frailty score (GFl $\geq 5)$. Of the 393 older people who were eligible for the study (frail and written informed consent) 47 older people were not included in the study, as they had not completed the baseline measurement (Figure 2). Finally, 346 older people were included in the study; 193 (56\%) of whom received the PoC approach. The mean age of participants was 77.2 years $(S D=5.1), 58 \%(n=199)$ were female, $49 \%$ $(n=170)$ were living alone and 58\% $(n=202)$ had a low level of education.
Table 1 Baseline characteristics of participants in control group and intervention group. Values are numbers (percentages) unless stated otherwise

\begin{tabular}{|l|c|c|}
\hline Characteristics & Control $(\mathbf{n = 1 5 3})$ & Intervention $(\mathbf{n = 1 9 3})$ \\
\hline Mean (SD) age (years) & $76.80(4.92)$ & $77.49(5.28)$ \\
\hline Female sex & $93(61)$ & $106(55)$ \\
\hline Living alone & $80(52)$ & $90(47)$ \\
\hline Low education & $94(61)$ & $108(56)$ \\
\hline Mean $(S D)$ scores: & & \\
\hline GARS total & $30.58^{*}(10.62)$ & $33.09^{*}(11.52)$ \\
\hline GARS ADL scale & $16.54^{*}(5.35)$ & $17.97^{*}(6.14)$ \\
\hline GARS IADL scale & $14.03(5.86)$ & $15.12(5.96)$ \\
\hline MSPP-CP-D & $1.90(1.63)$ & $1.63(1.48)$ \\
\hline MSPP-CP-F & $0.46(0.44)$ & $0.36(0.35)$ \\
\hline MSPP-FSP-D & $0.73(0.88)$ & 0.610 .84 \\
\hline MSPP-FSP-F & $0.45(0.63)$ & $0.38(0.55)$ \\
\hline Short FES-I & $12.38(4.72)$ & $13.24(5.39)$ \\
\hline HADS-D & $6.69(4.35)$ & $6.54(3.77)$ \\
\hline SSL-112 & $27.46(6.06)$ & $27.17(6.30)$ \\
\hline GFI & $6.72^{*}(1.71)$ & $7.13^{*}(1.89)$ \\
\hline PMS & $21.41(4.25)$ & $21.97(4.01)$ \\
\hline
\end{tabular}

GARS $=$ Groningen Activity Restriction Scale (range total scale 18-72, range activities of daily living (ADL) scale 11-44, range instrumental ADL (IADL) scale 7-28; higher scores indicate more disability); MSPP= Maastricht Social Participation Profile; MSPP-CP-D=MSPP consumpative participation, diversity score (range 0-7; higher score indicates more diverse consumptive participation); MSPP-CP-F=MSPP consumptive participation, frequency score (range $0-3$; higher score indicates more frequent consumptive participation); MSPP-FSP-D=MSPP formal social participation, diversity score (range 0-7; higher score indicates more diverse formal social participation); MSPP-FSP-F=MSSP formal social participation, frequency score (range $0-3$; higher score indicates more frequent formal social participation); Short FES-I=Short Falls Efficacy Scale-International (range 7-28; higher score indicates more fear of falling); HADS-D=Hospital Anxiety and Depression Scale-depression subscale (range $0-21$, higher score indicates more depressive); SSL-112=Social Support List-Interaction version (range 12-48; higher score indicates more social support); GFI=Groningen Frailty Indicator (range 0-15; higher score indicates more severe frailty); PMS=Pearlin Mastery Scale (higher score indicates less own control).

'Significant differences: $P<0.05$ 


\begin{tabular}{|c|c|}
\hline \multicolumn{2}{|c|}{ General practices $(n=12)$} \\
\hline$v$ & $\downarrow$ \\
\hline $\begin{array}{l}\text { Allocated to PoC approach ( } n=6 \text { practices) } \\
\text { Approached }(n=1,825) \\
\text { Returned questionnaire }(n=1,477,81 \%) \\
\text { Consent }(n=569,39 \%) \\
\text { Screening positive }(n=214,38 \%) \\
\text { Baseline completed/ included }(n=193,90 \%) \\
\text { Dropped out before baseline }(n=21) \text { : } \\
\text { - Death }(n=1) \\
\text { - Admission }(n=0) \\
\text { - Health problems }(n=5) \\
\text { - Lost interest }(n=6) \\
\text { - Other reasons }(n=9)\end{array}$ & $\begin{array}{l}\text { Allocated to care as usual ( } n=6 \text { practices) } \\
\text { Approached }(n=1,673) \\
\text { Returned questionnaire }(n=1,313,79 \%) \\
\text { Consent }(n=532,41 \%) \\
\text { Screening positive }(n=179,34 \%) \\
\text { Baseline completed/included }(n=153,85 \%) \\
\text { Dropped out before baseline }(n=26) \text { : } \\
\text { - Death }(n=4) \\
\text { - Admission }(n=4) \\
\text { - Health problems }(n=6) \\
\text { - Lost interest }(n=7) \\
\text { - Other reasons }(n=5)\end{array}$ \\
\hline$\vee$ & $\downarrow$ \\
\hline $\begin{array}{l}6 \text { month follow-up }(n=171,89 \%) \\
\text { Lost to follow-up }(n=22): \\
\text { - Death }(n=4) \\
\text { - Admission }(n=3) \\
\text { - Health problems }(n=5) \\
\text { - Lost interest }(n=6) \\
\text { - Other reasons }(n=4)\end{array}$ & $\begin{array}{l}6 \text { month follow-up }(n=145,95 \%) \\
\text { Lost to follow-up }(n=8): \\
\text { - Death }(n=2) \\
\text { - Admission }(n=1) \\
\text { - Health problems }(n=2) \\
\text { - Lost interest }(n=2) \\
\text { - Other reasons }(n=1)\end{array}$ \\
\hline$\vee$ & $\downarrow$ \\
\hline $\begin{array}{l}12 \text { month follow-up }(n=157,81 \%) \\
\text { Lost to follow-up }(n=14): \\
\text { - Death }(n=5) \\
\text { - Admission }(n=2) \\
\text { - Health problems }(n=4) \\
\text { - Lost interest }(n=2) \\
\text { - Other reasons }(n=1)\end{array}$ & $\begin{array}{l}12 \text { month follow-up }(n=141,92 \%) \\
\text { Lost to follow-up }(n=4): \\
\text { - Death }(n=1) \\
\text { - Admission }(n=1) \\
\text { - Health problems }(n=1) \\
\text { - Lost interest }(n=1) \\
\text { - Other reasons }(n=0)\end{array}$ \\
\hline$\vee$ & $\downarrow$ \\
\hline $\begin{array}{l}24 \text { month follow-up }(n=143,74 \%) \\
\text { Lost to follow-up }(n=14): \\
\text { - Death }(n=6) \\
\text { - Admission }(n=3) \\
\text { - Health problems }(n=3) \\
\text { - Lost interest }(n=0) \\
\text { - Other reasons }(n=2)\end{array}$ & $\begin{array}{l}24 \text { month follow-up }(n=127,83 \%) \\
\text { Lost to follow-up }(n=14) \text { : } \\
\text { - Death }(n=7) \\
\text { - Admission }(n=3) \\
\text { - Health problems }(n=1) \\
\text { - Lost interest }(n=3) \\
\text { - Other reasons }(n=0)\end{array}$ \\
\hline
\end{tabular}

We found significant differences between intervention and control group participants with regard to frailty $(\mathrm{GFI})$ and disability (GARS) scores. The intervention group participants were significantly frailer (GFI score 7.13 vs. $6.72, p<0.05)$ and more disabled (GARS score 33.09 vs. 30.58, $\mathrm{p}<0.05)$. All other characteristics were similar between the groups at baseline (Table 1). In total, 76 older people were lost to follow-up during the trial, significantly more people in the intervention group ( $26 \% \mathrm{vs}$ $17 \%, p<0.05)$

Primary outcome

All twelve clusters, consisting of 310 frail older people with a baseline disability score and at least one out of three follow-up measurements, were included in the mixed model multilevel analyses. With regard to disability, we identified no significan difference between control and intervention group at 24 months follow-up. We found no significant group by time interaction effects for the total GARS scores and for the $A D L$ and IADL subscale scores. After removing the interaction term from the model, we tested the trend for time. Both groups increased significantly $(p<0.05)$ in disability over a period of 24 months, but no significant differences between the groups with respect to their increase existed. Table 2 gives a summary of these results.

Secondary outcomes

Table 3 shows the results on the secondary outcomes. Again, we found no significan group by time interaction effects of the intervention group on any of these outcomes.

\section{Subgroup analyses}

The fixed effects for exposure groups (low versus high) by time interactions were no significant $(p<0.05)$. We found no significant $(p<0.05)$ mediating effects for a higher level of mastery or a lower level of frailty (data not presented).

Figure 2 Flow of participants through trial.

PoC $=$ Prevention of Care approach 

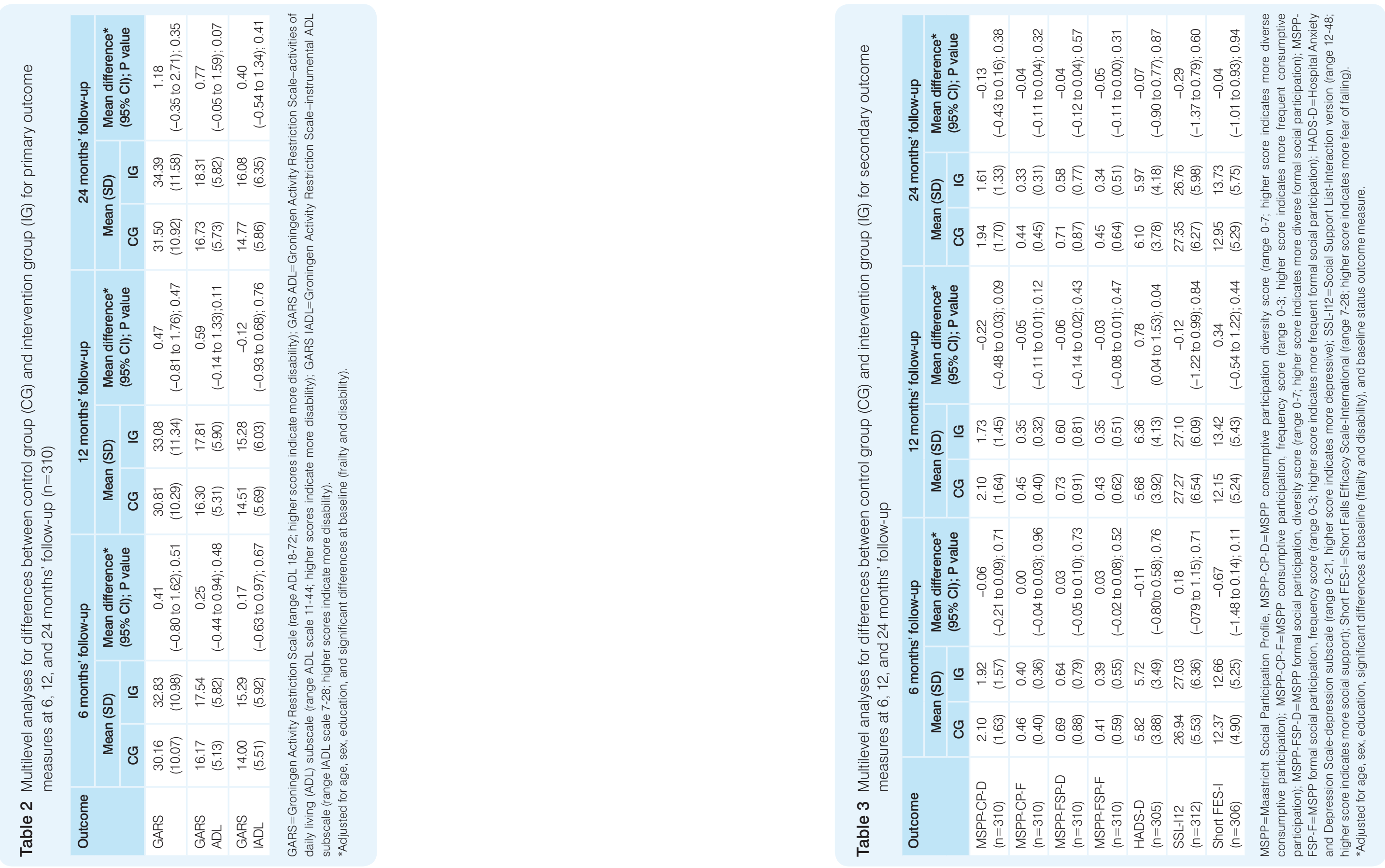


\section{Discussion}

Our study has provided no evidence for the effectiveness of a pro-active primary care approach, consisting of a multidimensional assessment with interdisciplinary care based on a tailor-made treatment plan and regular evaluation and follow-up, among frail older people. We found no significant differences between the intervention and control group (care as usual) with regard to disability (primary outcome) and our secondary outcomes: depressive symptomatology, social support interactions, fear of falling and social participation. Pre-planned subgroup analyses confirmed these results.

Strengths and weaknesses of study

The strengths of this cluster randomised trial include a long follow-up period with relatively few missing data and high follow-up rates. In addition, outcome measures with good psychometric properties were used, which were assessed by blinded data collectors. This study also has some weaknesses. Firstly, significant baseline differences existed between the intervention and control group with regard to frailty and disability and the sample size distribution was skewed. These differences were result of the cluster randomised design of the study, which is a common approach with this kind of intervention to avoid contamination bias..$^{17}$ Although, we adjusted for baseline differences in our analyses, this still may have affected our findings to some extent. Secondly, significantly more participants were lost to follow-up in the intervention than in the control group (26\% vs. $17 \%$ ). We cannot fully explain this finding, but older people in the intervention group were significantly more frail and disabled than those in the control group, which might have had affect the completion rate. Third, the PoC approach is evaluated in a real life setting among twelve GP practices. Although we did a comprehensive process evaluation alongside the trial, ${ }^{32}$ we have limited insigh into what has happened in practice for several reasons. Participating older people were patients of their GP irrespective of their study participation. Consequently making a distinction between usual care activities and contacts related to the PoC approach was difficult, resulting in an overlap in time spent in delivering usual care versus the new approach. Also, practice nurses had trouble in determing a clear endpoint of the PoC approach, because older people remained a patient of their GP after the PoC approach had been delivered, resulting in continuous monitoring of older people. In addition, older people were referred to other healthcare professionals as well. As a result, we do not know exactly how much time was spent in delivering the PoC approach and for how long these activities were continued. However, the process evaluation showed that slightly more than one third of the participants in the intervention group (34\%) had only the multidimensional assessment conducted by the practice nurse during an initial home visit. The remaining older people received tailor-made treatment followed by up to five follow-up visits by the practice nurse. ${ }^{32}$
Comparison with other studies

During the past decades, much research targeting community-dwelling (frail) older people has been done, with many studies in the field of preventive home visiting programs. Since 2000, several meta-analyses, ${ }^{33,34}$ systematic reviews, ${ }^{35-37}$ and literature reviews ${ }^{12,13}$ were published. The studies evaluated a range of interventions (such as multidimensional geriatric assessment, care planning, organisation and monitoring health promotion, self-management support, nursing services and referrals to other services) carried out by various professionals (GPs, nurses, allied professionals). The aim of these interventions is to proactively detect modifiable risk factors and worsening health conditions to reduce or prevent disability, health care use and related costs. Results regarding the effectiveness of these interventions have been inconsistent and conflicting. A few studies have shown favourable effects with regard to disability. For example, Bernabei and colleagues (1998) did a randomised controlled trial showing that a model of integrated care and case management had favourable effects with regard to disability in community-dwelling older people. ${ }^{38}$ Services were provided by the GP and a community geriatric evaluation unit, consisting of a geriatrician, a socia worker, and several nurses. Gill and colleagues (2002) have reported a successfu randomised controlled trial evaluating an intense exercise programme for physically frail older people living in the community. ${ }^{39}$ The programme is based on the outcomes of an extensive assessment and focus on the individual needs of older people, but also takes their environmental conditions into account. Most studies however, reported no or only modest effects of their interventions. Also, the largest trial in this field, by Fletcher and colleagues (2004), comparing different strategies for assessment (targeted versus universal) and evaluation and management (primary care versus multidisciplinary geriatric team) in more than 40,000 older people, did not result in convincing effects or adequate evidence that one strategy is better than another. ${ }^{40}$

Meaning of the study: explanations and clinical implications

Besides the methodological drawbacks, some other explanations for the lacking effects are possible. These relate to the target group, insufficient implementation of the PoC approach, and current healthcare delivery in the Netherlands.

Firstly, the PoC approach focuses on frail older people (GFl $\geq 5$ ). The baseline disability score in our frail sample (mean 32.0, $S D=11.2$ ) was substantially higher than in a comparable sample ( $\geq 70$ years) of the Dutch general population (mean 24.9 $\mathrm{SD}=9.3){ }^{41}$ Some participants in our study may have been too frail, as some previous reviews in the field of preventive home visiting programmes suggest that interventions may be more effective in low-risk, non-disabled older people. ${ }^{25,34}$ This is in line with a more recent review reporting that frail older people have to be identified in relatively early stage when negative health outcomes can still be avoided. ${ }^{42}$ In contrast, practice nurses and GPs interviewed during the process evaluation mentioned that a large 
number of participants were in their view not eligible for the PoC approach, as they had hardly any disability in terms of ADL and IADL. ${ }^{32}$ In efforts to reduce disability and prevent (further) functional decline, which older people would benefit the most from interventions such as the PoC approach is still not clear.

Secondly, the process evaluation showed that some parts of the intervention protocol were not implemented as planned. ${ }^{32}$ The problem analysis and the development of a preliminary treatment plan (step 3) was often not done in a bilatera or an extended team meeting and only half of the treatment plans were discussed with the frail older person (step 4). Also, the toolbox parts were not frequently used in the treatment phase (step 5), and the extent of evaluation and follow-up, especially among the healthcare professionals, was limited (step 6). ${ }^{32}$ Insufficient implementation is a well-known problem, especially in the field of preventive and behavioural change interventions. ${ }^{43}$ During the process evaluation, professionals mentioned that some parts of the intervention protocol were (too) time-consuming or difficult to apply:32 this may have been a reason for insufficient implementation. ${ }^{43}$ In addition, professionals expressed a need for more training on the job and more opportunities to exchange experiences with each other. Education and experience of professionals and the intensity of provided training activities are strongly related to beneficial outcomes.12 Despite an extensive development period and a comprehensive training program with regard to the intervention protocol, we probably failed in providing professionals with the needed competencies and feasible tools to apply rather complex concepts, such as interdisciplinary collaboration, tailor-made care and self-management support, into daily practice. ${ }^{32}$ For example, the development of individualised goals, a prerequisite for tailor-made care and self-management support, is a challenging task, as patients tend to adopt a passive role in goal setting. ${ }^{44}$ Encouragement of active involvement is even more difficult in older people owing to highly prevalent cognitive impairments, communication difficulties and comorbidities and as such requires a unique set of competencies. ${ }^{45}$ Goal identification tools, such as the Canadian Occupational Performance Measure ${ }^{46}$ or Goal Attainment Scaling ${ }^{47}$, may be useful in the process of goal setting. In addition, more attention has to be paid to the implementation of evaluation and follow-up activities, as a minimum intensity and length of follow-up is needed to reach favourable effects. ${ }^{25,34}$

Third, standard healthcare delivery in the Netherlands is already at a relatively high level. Nearly all people are covered by healthcare insurance, healthcare is easily accessible and its quality is often considered to be good. ${ }^{48}$ Moreover, the contrast between the PoC approach and care as usual was probably too small to detect substantial effects. The non-effective results of this study and the complexity of effective interventions (or elements) described in the literature mean that drawing conclusions about which specific strategies would result in a surplus effect in which target group is difficult.
Future research

Although this study has not shown any beneficial effects of a pro-active primary care approach, including a multidimensional assessment and interdisciplinary care based on a tailor-made treatment plan and regular evaluation and follow-up, it adds to the evidence base for clinical decision-making and future research regarding communitybased care for frail older people. The publication of non-effective studies is highly relevant to prevent an overestimation of the benefits of interventions and a waste of healthcare resources. This study contributes to the emerging body of evidence that more research is needed to improve the effectiveness of interventions for frail older people.

\section{Acknowledgements}

The authors would like to thank the participants in the study; participating healthcare professionals; Centre for Data and Information Management (MEMIC): Anita Legtenberg, Alfons Schroten, Marlène Ronner; the members of the research group: Lilo Crasborn (MCC Omnes), Simone Denis (MCC Omnes), Marlou Wolters (MCC Omnes), Herbert Habets (Orbis Medical Centre), Ramon Daniels (Zuyd University of Applied Sciences, Heerlen); their research assistants: Floor Koomen, Ine Hesdahl and Astrid Dello; and their sponsor: Dutch National Care for the Elderly Programme by The Netherlands Organisation for Health Research and Development (ZonMw 311070301), the Hague, the Netherlands. 


\section{References}

Bergman $\mathrm{H}$, Béland $\mathrm{F}_{\text {., }}$ Perrault A. The global challenge of understanding and meeting the needs of the frail older population. Aging Clin Exp Res. 2002;14(4):223-5.

2. Coleman EA. Challenges of systems of care for frail older persons: the United States of America experience. Aging Clin Exp Res. 2002:14(4):233-8.

3. Ploeg J, Brazil K, Hutchison B, Kaczorowski J, Dalby DM, Goldsmith CH, Furlong W. Effect of preventive primary care outreach on health related quality of life among older adults at risk of functional decline: nom

4. Woolhandler S, Campbell T, Himmelstein DU. Costs of health care administration in the United States and Canada N Engl J Med. 2003;349:768-75

5. Keating N, Ottinowski P, Wenger C, Fast J, Derksen L. Understanding the caring capacity of informal etworks of frail seniors: a case for care networks. Ageing Soc. 2003;23(1):115-27.

6. Markle-Reid M, Browne G, Weir R, Gafni A, Roberts J, Henderson SR. The effectiveness and efficiency of home-based nursing health promotion for older people: review of the literature. Med Care Res Rev. 2006;63(5):531-69.

7. Stijnen MMN, Duimel-Peeters IGP, Jansen MWJ, Vrijhoef HJM. Early detection of health problems in potentially frail community-dwelling older people by general practices - project [G]OLD: design of a ongitudinal, quasi-experimental study. BMC Geriatr. 2013;13(7):1-10.

8. Fletcher AE, Jones DA, Bulpitt CJ, Tulloch AJ. The MRC trial of assessment and management of older people in the community: objectives, design and interventions [ISRCTN23494848]. BMC Health Serv

9. Fried $L P$, Ferrucci L, Darer J, Williamson JD, Anderson G. Untangling the concepts of disability, frailty and comorbidity: implications for improved targeting and care. J Gerontol A Biol Sci Med Sel. 2004;59(3):255-63.

10. Markle-Reid M, Browne G Conceptualizations of frailty in relation to older adults. J Adv Nurs. 2003:44(1):58-68

11. Pel Littel RE, Schuurmans MJ, Emmelot Vonk MH, Verhaar HJJ. Frailty: defining and measuring of a concept. J Nutr Health Aging. 2009;13(4):390-4

12. Liebel DV, Friedman B, Watson NM, Powers BA. Review of nurse home visiting interventions for communitydwelling older persons with existing disability. Med Care Res Rev. 2009;66(2):119-46.

13. Hallberg IR, Kristensson J. Preventive home care of frail older people: a review of recent case management studies. J Clin Nurs. 2004;13(6B):112-20.

14. Daniels R, Metzelthin S, Van Rossum E, De Witte L, Van den Heuvel W. Interventions to prevent disability in frail community-dwelling older persons: an overview Eur J Ageing 2010;7(1):37-55.

15. Daniels R, van Rossum E, Metzelthin S, Sipers W, Habets H, Hobma S, van den Heuvel W, de Witte L. A disability prevention programme for community-dwelling frail older persons. Clin Rehabil. 2011;25(11):963-74.

6. Metzelthin SF, van Rossum E, de Witte LP, Hendriks MR, Kempen Gl. The reduction of disability in community-dwelling frail older people: design of a two-arm cluster randomized controlled trial. BMC Public Health. 2010;10:51

17. Campbell MK, Elbourne DR, Altman DG, and CONSORT group. CONSORT statement: extension to cluster randomised trials. BMJ. 2004;328(7441):702-8.

18. Steverink N, Slaets JPJ, Schuurmans $\mathrm{H}$, van Lis M. Measuring frailty: development and testing of the Groningen Frailty Indicator (GFI). Gerontologist. 2001;41(1):236-7.

19. DerckX E. Eerste nurse practitioners met differentiatie huisartsenzorg. Tijdschr Verpleegkundigen 2006;3:26-30

20. Van Weel C, Schers H, Timmermans A. Health Care in The Netherlands. J Am Board Fam Med. 2012;25(Suppl 1):S12-7.

21. Daniels R. Frail Elderly - Identification and disability prevention in primary care [PhD Thesis]. Maastricht School for Public Health and Primary Care, Maastricht University; 2011

22. Glasgow RE, Emont S, Miller DC. Assessing delivery of the five 'As' for patient-centred counseling Health Promot Int. 2006;21(3):245-55.
23. Miller WR, Rollnik S. Motivational Interviewing: preparing people for change. New York: Guildford Publications; 2002

24. Kempen GIJM, Miedema I, Ormel J, Molenaar W. The assessment of disability with the Groningen Activity Restriction Scale. Conceptual framework and psychometric properties. Soc Sci Med 1996;43(11):1601-10.

25. Stuck AE, Walthert JM, Nikolaus T, Bula CJ, Hohmann C, Beck JC. Risk factors for functional status decline in community-living elderly people: a systematic literature review. Soc Sci Med. 1999:48(4):445-69.

26. Spinhoven P, OrmelJ Sloekers PPA, Kempen GIJM, Speckens AEM, van Hemert AM A validation sudy

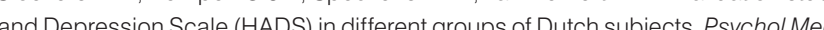
1997;27(2):363-70

27. Kempen GIJM, van Eijk LM. The psychometric properties of the SSL12-I, a short scale for measuring social support in the elderly. Soc Indic Res 1995;35(3):303-12.

28. Kempen GIJM, Yardley L, van Haastregt JCM, Zillstra GAR, Beyer N. Hauer K, Todd C. The Short FES-I. a shortened version of the falls efficacy scale-international to assess fear of falling. Age Ageing 2008;37(1):45-50.

29. Mars GMJ, Kempen GIJM, Post MWM, Proot IM, Mesters I, van Eijk JTM. The Maasticht Socia Particiaption Profile: development and clinimetric properties in older adults with a chronic physical illness. Qual Life Res. 2009;18(9):1207-18.

30. Pearlin LI, Schooler C. The structure of coping. J Health Soc Behav. 1978:19(1):2-21.

31. Kempen GIJM. Preventie en ouder worden: mogelijkheden voor zelfmanagement. Epid Bull. 2006;41(2):27-32

32. Metzelthin SF, Daniels R, Van Rossum E, Cox K. Habets H, de Witte LP, Kempen GIJM. A nurse-led interdisciplinary care approach to prevent disability among community-dwelling frail older people: large-scale process evaluation. Int J Nurs Stud. 2013;50 (9):1184-96.

33. Elkan R, Kendrick D, Dewey M, Hewitt M., Robinson J., Blair M., Williams D., K. B. Effectiveness of home based support for older people: systematic review and meta-analysis. BMJ. 2001;323(7315):719-25.

34. Stuck AE, Egger M, Hammer A, Minder CE, Beck JC. Home visits to prevent nursing home admission and functional decline in elderly people: systematic review and meta-regression analysis. JAMA $2002 \cdot 287(8) \cdot 1022-8 \cdot(-10)$

35. Bouman A, van Rossum E, Nelemans P., Kempen GIJM, Knipschild P. Effects of intensive home visiting programs for older people with poor health status: a systematic review. BMC Health Serv Res. 2008;8:74.

36. Huss A, Stuck AE, Rubenstein LZ, Egger M, Clough-Gorr KM. Multidimensional preventive home vis programs for community-dwelling older adults: a systematic review and meta analysis of randomized controlled trials:: A Systematic Review Biol Sci Med Sci. 2008;63(3):298-307.

37. Van Haastregt JC, Diederiks JP, van Rossum E, de Witte LP, Crebolder HF. Effects of preventive hom visits to elderly people living in the community. systematic review. BMJ. 2000;320(7237):754-8

38. Bernabei R, Landi F, Gambassi G, Sgadari A, Zuccala G, Mor V, Rubenstein LZ, Carbonin P. Randomised trial of impact of model of integrated care and case management for older people living in the community. BMJ. 1998;316(7141):1348-51.

39. Gill TM, Baker DI, Gottschalk M, Peduzzi PN, Allore H, Byers A. A program to prevent functional decline in physically frail, elderly persons who live at home. N Engl J Med. 2002;347(14):1068-74.

40. Fletcher AE, Price GM, Ng ESW, Stirling SL, Bulpitt CJ, Breeze E, Nunes M, Jones DA, Latif. A., Fasey NM, Vickers MR, Tulloch AJ. Population-based multidimensional assessment of older people in UK general practice: a cluster-randomised factorial trial. Lancet. 2004;364(9446):1667-77.

41. Daniels R, Van Rossum HIJ, Beurskens A, Van den Heuvel W, De Witte L. The predictive validity of thre self-report screening instruments for identifying frail older people in the community. BMC Public Health 2012;12:69.

42. Pijpers E, Ferreiral, Stehouwer CDA, Nieuwenhuijzen Kruseman AC. The frailty dilemma. Review of the predictive accuracy of major frailty scores. Eur J Intern Med. 2012;23(2):118-23.

43. Glasgow RE, Emmons KM. How can we increase transiation of research into practice? Types of evidence needed. Annu Rev Public Health. 2007:28:413-33. 
44. Siegert RJ, Taylor WJ. Theoretical aspects of goal-setting and motivation in rehabilitation. Disabil Rehabil. 2004:26(1):1-8

45. Parsons JGM, Parsons MJG. The effect of a designated tool on person-centred goal identification and service planning among older people receiving homecare in New Zealand. Health Soc Care Community 2012;20(6):653-62.

46. Law M, Baptiste S, McColl M, Opzoomer A, Polatajko H, Pollock N. The Canadian Occupationa .

4. Kiresuk TJ, Sherman RE. Goal Attainment Scaling: a general method for evaluating comprehensive community mental health programs. Community Ment Health 1968; (4):443-53. 4. Smeulders ESTF, van Haastregt JCM, Ambergen T, Uszko-Lencer NHKM, Janssen-Boyne JJJ, Gorge APM, Stoffers HEJH, Lodewijks-van der Bolt CLB, van Eijk JTM, Kempen GIJM. Nurse-led sel$J$ Adv Nurs. 2010:66(7):1487-99. 
Cost-effectiveness of an interdisciplinary primary care approach to reduce disability in community-dwelling frail older people: a cluster randomised controlled trial

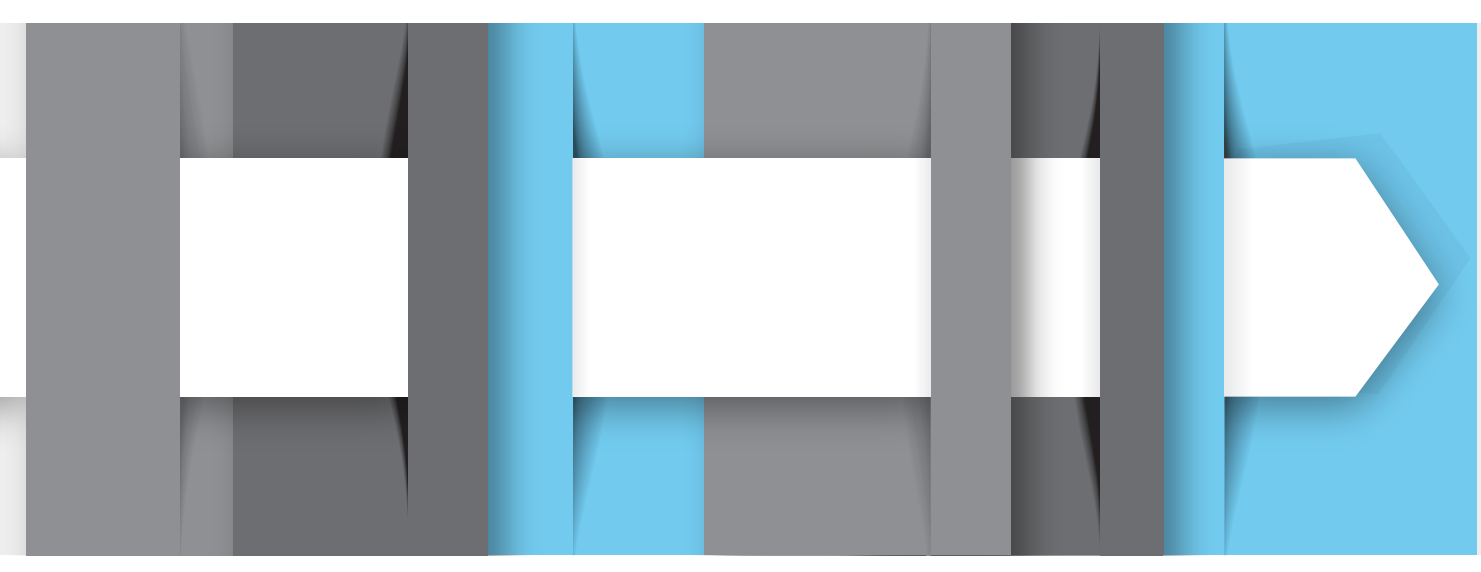

Under review

Metzelthin, SF, van Rossum, E, Hendriks, MRC, de Witte, LP, Hobma, SO Sipers, W, Kempen, GlJM 


\section{Abstract}

Background: There is increasing interest in cost-effective interventions for community dwelling frail older people. However, there are only few economic evaluations conducted. The aim of this study was to evaluate the cost-effectiveness of an interdisciplinary primary care approach

Methods: Embedded in a cluster randomised trial among 12 general practitioner practices (registered with Current Controlled Trials, ISRCTN31954692), an economic evaluation was performed from a societal perspective with a time horizon of 24 months. Frail older people in the intervention group received a multidimensional comprehensive geriatric assessment and interdisciplinary care based on a tailor made treatment plan and regular evaluation and follow-up. Practices in the contro group delivered usual care. The primary outcome for the cost-effectiveness (CEA) and cost-utility analysis (CUA) was disability and quality of life, respectively.

Results: Multilevel analyses among 346 frail older people showed no significant differences between the groups regarding disability and quality of life at 24 months. People in the intervention group used, as expected, more primary care services, but there was no decline in more expensive hospital and long-term care. Total costs over 24 months tended to be higher in the intervention group than in the control group (€26,503 versus $€ 20,550, p=0.08$ ).

Conclusion: This study does not support the surplus value of the interdisciplinary primary care approach for frail older people and its implementation in the curren form is not recommended, at least in the Netherlands or other countries with similar healthcare systems.

\section{Background}

Frail older people have an increased risk for adverse outcomes such as disability.1, In most Western countries, including the Netherlands and the US, disability prevention in community-dwelling frail older people is considered to be a priority in geriatric care and research. ${ }^{3,4}$ In several countries, for example the UK, proactive approaches, including early detection and treatment of frail older people, have even became part of the national healthcare policy. ${ }^{5}$ However, results regarding their effectiveness are inconsistent and little is known about their cost-effectiveness. ${ }^{6-9}$ The review of Markle-Reid and colleagues ${ }^{9}$ identified six studies between 1966 and 2003 that included an economic evaluation; three studies provided some evidence that healthcare costs can be reduced (in particular by hospital and nursing home savings). However, the usefulness of this evidence is limited as only one study ${ }^{10}$ conducted a cost-effectiveness analysis, meaning that relative costs and outcomes (effects) were compared. During the last decade (2003-2013) a few more economic evaluation were conducted, ${ }^{11-14}$ but only one study ${ }^{14}$ showed some beneficial effects regarding functional decline and improved well-being at 6 months, while costs were comparable between both groups. Long-term effects are not known. ${ }^{14}$

Between 2008 and 2010 Daniëls and colleagues ${ }^{15}$ developed an interdisciplinary primary care approach for frail older people: the 'Prevention of Care' $(\mathrm{PoC})$ approach.' It was expected that PoC leads to reduced disability and improved quality of life in frail older people. Furthermore, we assumed an increase in the use of primary care services and a decline in the use of more expensive hospital and long-term care, leading to a reduction in overall healthcare costs, and consequently a cost-effective intervention. This paper reports about shifts in healthcare utilisation and the costeffectiveness of the PoC approach compared to usual care.

\section{Methods}

\section{Design}

Embedded in a cluster randomised controlled trial a cost-effectiveness (CEA) and cost-utility analysis (CUA) were performed from a societal perspective with a time horizon of 24 months. The design, methods, feasibility, and clinical outcomes of this trial have been described in detail elsewhere. ${ }^{17-19}$ The Medical Ethical Committee of Maastricht University/ Academic Hospital Maastricht approved the study (MEC 09-3-067). 
Setting and participants

The trial was conducted among 12 Dutch general practitioner (GP) practices. Community-dwelling frail older people ( $\geq 70$ years) were recruited from these practices by means of postal screening, including the Groningen Frailty Indicator (GFI). ${ }^{20}$ People with a GFI score of five or higher (range 0-15) who signed the informed consent prior to the baseline measurement were eligible for the study.

Randomisation and blinding

Before randomisation, GP practices were pre-stratified into four strata based on two characteristics: number of older patients ( $<350$ patients versus $\geq 350$ patients) and location (urban versus rural area). A computer generated randomisation list was used to allocate stratified pairs to either the intervention or control group..$^{17}$ Whereas participants and healthcare professionals were aware of the allocated arm, outcome assessors were kept blinded to the allocation.

Intervention

Older people in the intervention group received the PoC approach, which was delivered by an interdisciplinary team (e.g. GP, practice nurse, occupational therapist and physiotherapist). The six-step approach aims to reduce disability and to preven (further) functional decline ${ }^{16}$ After the frailty screening (step 1), people receive comprehensive in-home assessment by the practice nurse and additional assessments by other professionals if indicated (step 2). In a bilateral meeting (i.e. GP and practice nurse) or in an extended team meeting (e.g. GP, practice nurse, occupational therapist and physiotherapist) a preliminary treatment plan is formulated (step 3). During a second home visit a final treatment plan is made together with the frail older person (step 4). A specific toolbox offers recommendations and guidelines for the execution of the treatment plan (step 5). The achievement of goals and implementation of strategies in daily life are regularly evaluated and the need for follow-up is determined (step 6). The intervention is described in more detal elsewhere. ${ }^{16}$ Older people in the control group received usual care. In the Netherlands, GPs have a central position in community-based care, as they are gatekeepers to secondary care. In 2001, the profession of practice nurses was introduced to reduce the workload of GPs. ${ }^{21}$ However, no standard approach for the care for frail older people is available.

Healthcare utilisation and costs

We assessed intervention costs, other healthcare costs and patient and family costs. Intervention costs (i.e. screening, training activities and intervention delivery) were not assessed individually, but mean volumes were estimated based on the feasibility study that was conducted alongside this trial (see Appendix 1). ${ }^{19}$ Other healthcare costs related to: primary care, including services from GP, practice nurse, allied professionals (i.e., occupational therapist, physiotherapist, speech therapist and dietician), hospital care (i.e., admission days, outpatient medical services and day care), long-term care (i.e., home for the elderly/ nursing home admission, professional homecare), and prescribed medication. In addition, patient and family costs (i.e. informal care and helping aids/ in-home modifications) were assessed. During 24 months, volumes of healthcare utilisation as well as medication costs were collected from registries of health insurance agencies, as nearly all people in the Netherlands are covered by healthcare insurance. ${ }^{22}$ Volumes that could not be obtained from these registries were assessed from the local hospital or directly from the participants by means of telephone interviews and postal questionnaires at baseline, 6, 12 and 24 months follow-up (see Appendix 2).

Cost valuation was conducted according to Dutch guidelines for costing research. ${ }^{23}$ Costs were calculated by multiplying the volumes with cost prices of that unit (see Appendix 2). Where no standardised cost prices were available (i.e., helping aids and assistive devices), costs were obtained from the internet. Costs are presented in Euros (€) for the year 2010, and if needed, prices were indexed to the reference year using a consumer price index..$^{23}$

Cost-effectiveness

The primary outcome of our CEA is disability, which was assessed by the Groningen Activity Restriction Scale (GARS) ${ }^{24}$ at baseline, 6, 12, and 24 months. For the CUA outcomes are expressed in terms of generic quality adjusted life years (QALYS), measured by means of the EuroQol-5D (EQ-5D). ${ }^{25} \mathrm{~A}$ direct value for every state of health was generated using the UK Dolan tariff, ${ }^{26}$ which involves an algorithm for interpolating EuroQol results to population utilities. Utilities refer to preferences that individuals or society may have for a particular set of health outcomes. ${ }^{25}$ The utilities at the four measurement points were used to compute QALYs using the area under the curve method..$^{25}$ Data for CEA and CUA were collected at 6, 12, and 24 months.

Statistical analysis

Clinical outcomes were analysed according to the intention-to-treat principle using mixed model multilevel analyses. Volumes of healthcare utilisation and related costs are presented as arithmetic means and were tested for their significance by using t-tests, which is considered the most appropriate method to analyse cost data. ${ }^{27}$ Incremental cost-effectiveness ratios (ICERs) were calculated, representing the differences in mean costs between the intervention and control group in the numerato and the difference in mean clinical effects in the denominator. ${ }^{25}$ Sampling uncertainty around the ICER was assessed by means of (1,000 times) non-parametric bootstrapping (percentile method). ${ }^{25}$ To assess the robustness of the assumptions 
made, we performed one-way sensitivity analyses. First, the intervention costs were not assessed individually, but mean volumes were estimated based on the feasibility study that was conducted alongside this trial. ${ }^{19}$ If interventions aspects are reimbursed as usual care this may have resulted in double counting of intervention costs. Therefore, we compared the base-case with the analyses without intervention costs. Second, as the study was performed in a Dutch setting, the base-case (UK tariff) ${ }^{26}$ was compared with the Dutch tariff ${ }^{28}$. Statistical analyses were conducted with SPSS for Windows version 20.0, bootstrapping was done in Excel 2010.

\section{Results}

Participants

In total, 346 frail older people were included in the study 193 (56\%) received the PoC approach. The mean age of participants was 77.2 years. At baseline, participants in the intervention group were on average significantly more frail (GFI: 7.13 versus 6.72 , $p=0.03$ ) and more disabled (GARS: 33.09 versus 30.58, $p=0.03$ ). Other characteristics and the amount of healthcare utilisation were comparable between both groups (Table 1). Details of the progress of participants through the trial are reported elsewhere. ${ }^{18}$

Healthcare utilisation and costs

Volumes of hospital care (including outpatient medical services), long-term care, informal care and helping aids/ in-home modifications were comparable between the groups over a period of 24 months. However, people in the intervention group had more primary care use compared to the control group (Table 2).

Additional analyses of annual volumes showed that this group difference was strongest in the first 12 months: the intervention group showed significantly more contacts with the occupational therapist ( 4.6 to $0.4, \mathrm{p}<0.001)$, practice nurse (5.2 to $1.0, p<0.001)$ and GP (15.0 to $12.4, p=0.05)$. From month 12 to 24 only the amount of occupational therapy sessions was significantly higher in the intervention group (2.6 to $0.7, p=0.01$ ). Annual volumes are not tabulated, but available on request.

Table 3 shows the mean costs for participants with a complete dataset over a period of two years $(n=194,56 \%)$. The mean total costs in 24 months were $€ 26,503$ per participant in the intervention group compared to €20,550 in the control group. The costs for the PoC approach were $€ 728$. Mean healthcare costs were significantly higher in the intervention group than in the control group ( $€ 17,664$ versus $€ 12,963$, $p=0.03$ ). Participants in the intervention group showed significantly higher mean costs for GP care ( $€ 1,072$ versus $€ 729, p<0.001)$, hospital care ( $€ 2,905$ versus $€ 1.488$ $p=0.05$ ), and helping aids/ in-home modifications ( $€ 552$ versus $€ 278, p=0.03$ ) than
Table 1 Baseline characteristics of frail older people $(n=346)$

\begin{tabular}{|c|c|c|}
\hline & $\begin{array}{c}\text { Intervention } \\
(\mathrm{n}=193)\end{array}$ & $\begin{array}{l}\text { Control } \\
(n=153)\end{array}$ \\
\hline Age mean & 77.5 (5.3) & $76.8(4.9)$ \\
\hline Female & $106(55)$ & $93(60)$ \\
\hline Living alone & $90(47)$ & $80(52)$ \\
\hline Low education & $108(56)$ & $94(61)$ \\
\hline \multicolumn{3}{|l|}{ Frailty } \\
\hline GFI $\underline{0}-15^{* \dagger}$ & $7.1(1.9)$ & $6.7(1.7)$ \\
\hline \multicolumn{3}{|l|}{ Disability } \\
\hline GARS $\underline{18-72^{*+}}$ & $33.1(11.5)$ & $30.6(10.6)$ \\
\hline \multicolumn{3}{|l|}{ Utilities } \\
\hline EuroQol-5D based on UK tariff $(0-1)^{\dagger}$ & $0.6(0.2)$ & $0.7(0.2)$ \\
\hline EuroQol-5D based on NL tariff $(0-1)^{\dagger}$ & $0.7(0.2)$ & $0.7(0.2)$ \\
\hline Contacts GP last year ${ }^{\ddagger}$ & $6.5(5.2)$ & $4.5(3.8)$ \\
\hline Contacts allied professionals last year ${ }^{\ddagger}$ & $24.9(47.3)$ & $19.3(30.3)$ \\
\hline Hospital admission last year (days) $)^{\ddagger}$ & $3.4(10.1)$ & $3.3(16.1)$ \\
\hline Nursing home admission last year (days) & $0.2(3.0)$ & $2.0(14.2)$ \\
\hline Professional homecare (hours/week) & $2.4(3.4)$ & $2.2(3.2)$ \\
\hline Informal care (hours/week) & $7.0(20.9)$ & $5.1(10.0)$ \\
\hline Aids/ in-home modifications available & $3.2(2.6)$ & $2.9(2.7)$ \\
\hline \multicolumn{3}{|c|}{$\begin{array}{l}\text { Notes: Data are n (\%), or mean (SD); GFI=Groningen Frailty Indicator; GARS=Groningen Activity } \\
\text { Restriction Scale. } \\
\text { *Significant differences at baseline } p<0.05 \text {. } \\
\text { t Underlined scores=most favourable scores. } \\
\text { fBaseline: self-report, follow-up measurements: continuous registration. }\end{array}$} \\
\hline
\end{tabular}

participants in the control group. The remaining categories of healthcare utilisation were comparable between the groups. 

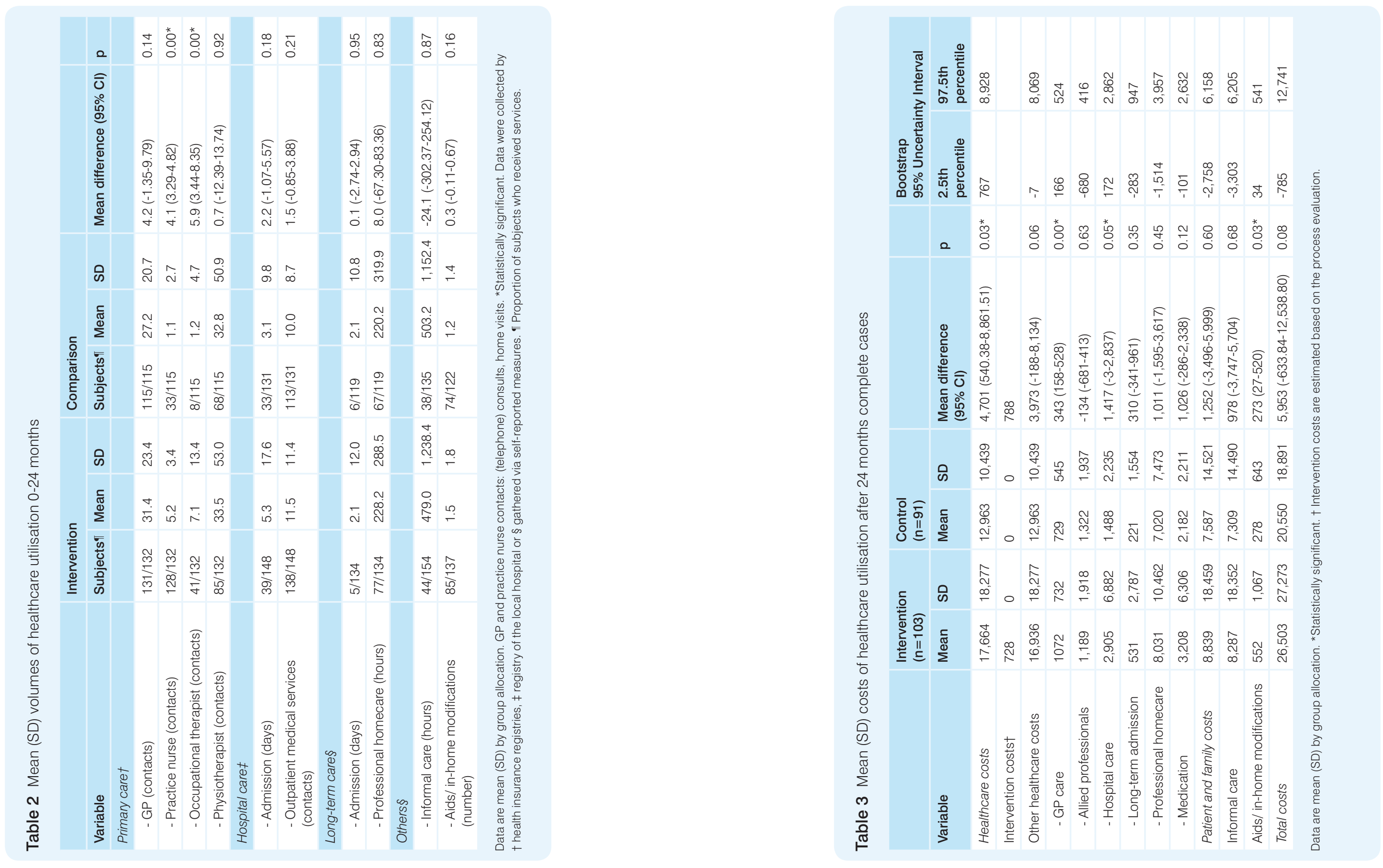


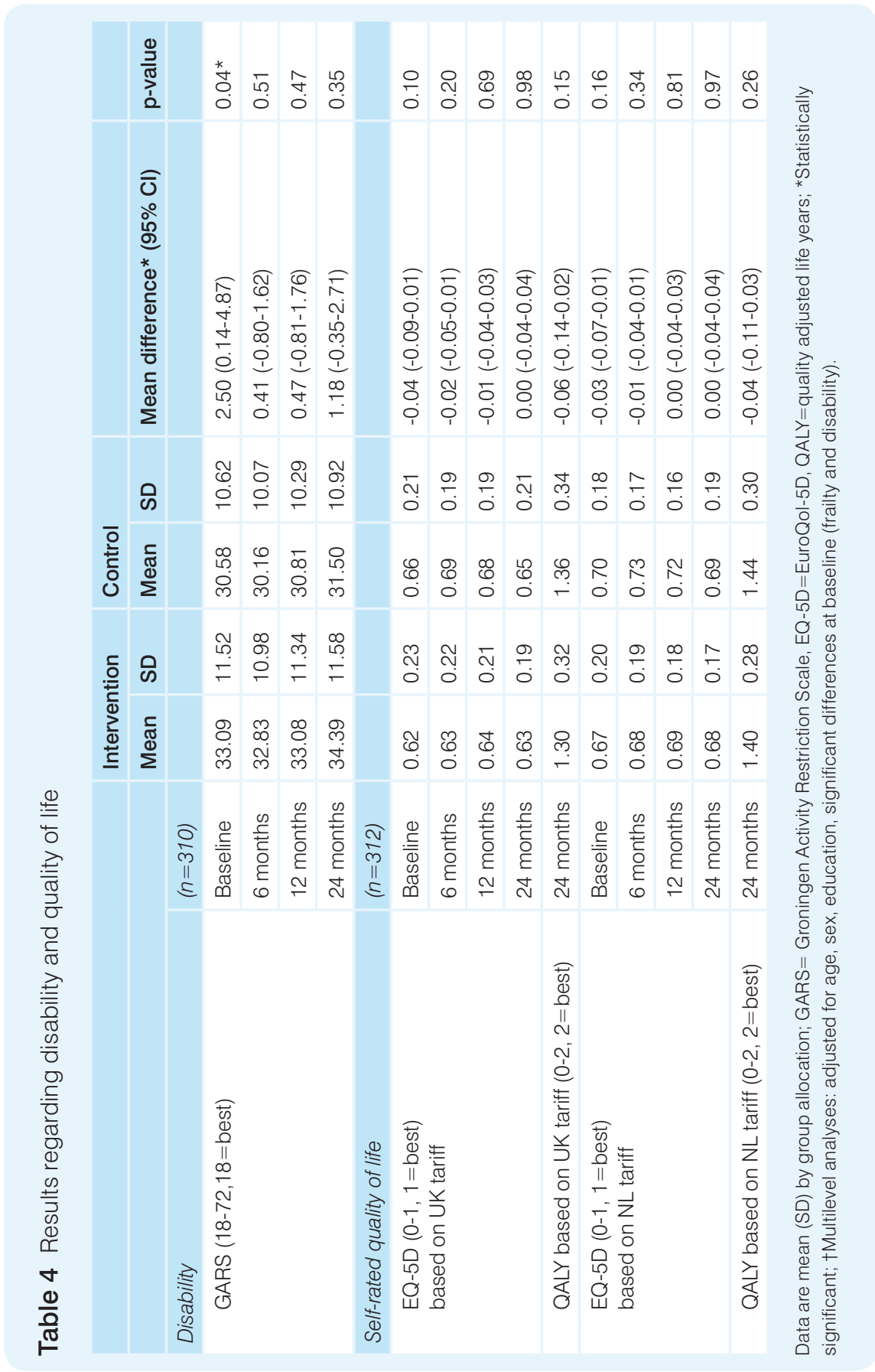

Clinical outcomes

No significant effects were found for disability (GARS) and quality of life (EuroQol-5D). Table 4 summarises scores at baseline, 6, 12 and 24 months follow-up. In addition 2 year QALYs are presented. ${ }^{29}$

Base-Case cost-effectiveness analysis and sensitivity analyses Since we did not detect differences in clinical effects, it is not useful to present ICERs. The cost-effectiveness plane of the CUA base-case bootstrap analysis for QALYS (Figure 1) shows that $2 \%$ of the ICERs lies in the dominant ('south east') quadrant (representing the probability of the PoC approach having more effect and lower costs compared to usual care). Sensitivity analyses did not reveal other results (see Appendix 3).

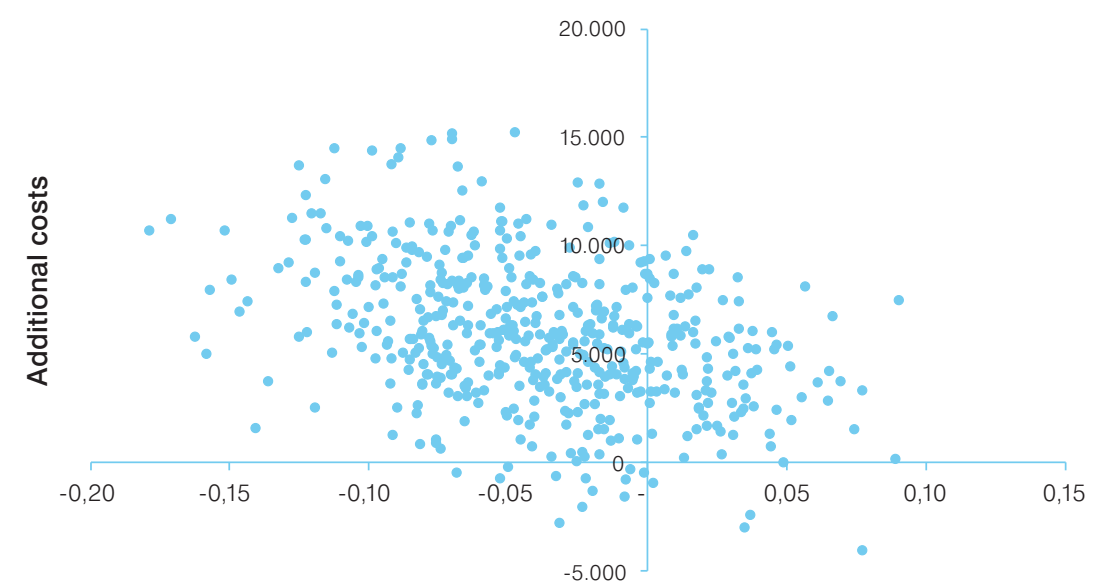

Additional effects

Figure 1 Cost-effectiveness plane for costs (euros) versus adjusted life years (QALYS) based on UK tariff

\section{Discussion}

As expected frail older people in the intervention group used more primary care services compared to the control group, especially in the first 12 months, but against our hypothesis we found no effects on disability or quality of life and no decline in more expensive hospital and long-term care. Subsequently, total healthcare costs 
over 24 months tended to be higher in the intervention group than in the control group (€26,503 versus $€ 20,550, p=0.08$ ). The probability of the PoC approach being cost-effective compared to usual care is negligible (2\%).

The findings of this economic evaluation are in line with the effect evaluation. ${ }^{18}$ There are some possible explanations why the PoC approach has shown no beneficia effects. First, there is still a lively debate about the conceptualisation and measurement of frailty. ${ }^{30}$ Possibly the wrong target group was recruited for the PoC approach. Second, according to the process evaluation some steps of the intervention protoco were not sufficient implemented.19 The problem analysis and development of a preliminary treatment plan (step 3) was often not done in a bilateral or an extended team meeting and only half of the treatment plans were discussed with the frail older person (step 4). Also toolbox parts were not frequently used in the treatment phase (step 5) and the extent of evaluation and follow-up, especially among the healthcare professionals, was limited (step 6)..$^{19}$ Finally, standard healthcare in the Netherlands is at a relatively high level. Nearly all people are covered by healthcare insurance and care is easily accessible. ${ }^{22}$ This is supported by the volumes of healthcare utilisation in the control group. Over a period of 24 months, people in this latter group had, on average, 27 contacts with their GP, 33 physiotherapy sessions and 10 contacts with outpatient medical services. The contrast between the PoC approach and usual care was possibly too small to demonstrate convincing effects. This is in line with other studies assuming that the increasing awareness of the needs and problems of older people might have reduced the potential benefit of recent interventions compared to studies in earlier decades. ${ }^{31}$

Comparing our results with previous studies is complex for several reasons First, interventions differ in terms of content, disciplines involved, duration, intensity and setting. Second, the measurement and data analysis of volumes of healthcare utilisation and related costs vary across studies. Third, results of economic evaluations have to be interpreted in the light of national contexts, as cost-effectiveness strongly depends on the characteristics of the healthcare system in which the intervention is evaluated. ${ }^{25}$ Finally, economic evaluations in this field are scarce. To our knowledge the present study is the first cost-effectiveness analysis of an interdisciplinary primary care approach for frail older people, including postal screening, a multidimensiona assessment and interdisciplinary care based on a tailor made treatment plan and regular evaluation and follow-up, which was conducted from a societal perspective with a time horizon of 24 months.

The present study has some limitations that may have influenced our results First, although healthcare registries were used as primary data sources, some data were collected via self-reports, which were assessed at 6, 12 and 24 months follow-up. The relatively long period between the measurements may have led to recall bias. However, there is no reason to assume that possible recall bias is unevenly distributed between the groups. Second, the intervention costs were not assessed individually, but mean costs were based on the process evaluation. ${ }^{19}$ When interventions aspects are reimbursed as usual care this may have resulted in double counting. However sensitivity analyses without intervention costs did not reveal other results though.

Against our hypothesis, the PoC approach is not cost-effective. Consequently, its implementation in its current form is not recommended, at least not in the Netherlands or other countries with similar healthcare systems. Anyway, it is questionable to some extent whether it is justified yet to integrate early detection and treatment of frail older people in national healthcare policies. According to the screening literature, the 'screening condition' has to be fully understood and suitable screening tools and accepted interventions are needed. ${ }^{32}$ Furthermore, the costs of screening, including subsequent assessment and treatment should be economically balanced in relation to potential healthcare costs. ${ }^{32}$ With regard to frailty there is, as mentioned earlier, still a lively debate about its conceptualisation and measurement. ${ }^{30}$ In addition, available interventions have produced inconsistent and conflicting results regarding their effectiveness and little is known about cost-effectiveness. ${ }^{6-9}$ At leas our study has shown that such a proactive approach may lead to increased healthcare costs without providing any beneficial effects. Consequently, more research into the conceptualisation and measurement of frailty is needed and more studies, including economic evaluations, have to be conducted to optimise services for communitydwelling frail older people.

Acknowledgements

We thank older people and professionals for their participation. We are grateful to the insurance agencies (ZilverenKruis/ Achmea, VGZ and CZ in the Netherlands) and the local hospital (Orbis Medical Centre Sittard-Geleen, the Netherlands) for providing healthcare utilisation data.

\section{Funding}

This work was supported by The Netherlands Organisation for Health Research and Development (ZonMw 311070301), the Hague, the Netherlands. 


\section{References}

1. Fried LP, Ferrucci L, Darer J, Williamson JD, Anderson G. Untangling the concepts of disability, frailty and comorbidity implications for improved targeting and care $J$ Gerontol A Biol Sci Med SCi. 2004;59(3):255-63

2. Fried LP, Tangen CM, Walston J, Newman AB, Hirsch C, Gottdiener J, Seeman T, Tracy R, Kop WJ . 2001;56(3):M146-56

3VZ. Preventie bij ouderen: focus op zelfredzaamheid. Den Haag: Gezondheidsraad2009.

4. Ferrucci L, Guralnik JM, Studenski S, Fried LP, Cutler GB, Jr., Walston JD. Designing randomized controlled trials aimed at preventing or delaying functional decline and disability in frail, older persons: a consensus report. J Am Geriatr Soc. 2004;52(4):625-34.

5. Fletcher AE, Price GM, Ng ESW, Stirling SL, Bulpitt CJ, Breeze E, Nunes M, Jones DA, Latif. A., Fasey NM, Vickers MR, Tulloch AJ. Population-based multidimensional assessment of older people in UK general practice: a cluster-randomised factorial trial. Lancet. 2004;364(9446):1667-77.

6. Bouman A, van Rossum E, Nelemans P., Kempen GIJM, Knipschild P. Effects of intensive home visiting programs for older people with poor health status: a systematic review. BMC Health Serv Res. $2008 \cdot 8 \cdot 74$

7. Huss A, Stuck AE, Rubenstein LZ, Egger M, Clough-Gorr KM. Multidimensional preventive home visit programs for community-dwelling older adults: a systematic review and meta analysis of randomized controlled trials. J Gerontol A Biol Sci Med Sci. 2008;63(3):298-307.

8. Stuck AE, Egger M, Hammer A, Minder CE, Beck JC. Home visits to prevent nursing home admission and functional decline in elderly people: systematic review and meta-regression analysis. JAMA 202;287(8):1022-8

9. Markle-Reid M, Browne G, Weir R, Gafni A, Roberts J, Henderson SR. The effectiveness and efficiency of home-based nursing health promotion for older people: review of the literature. Med Care Res Rev. 2006;63(5):531-69.

0. Stuck AE, Aronow HU, Steiner A, Alessi CA, Büla CJ, Gold MN, Yuhas KE, Nisenbaum R, Rubenstein ZZ, Beck JC. A trial of annual in-home comprehensive geriatric assessments for elderly people livin in the community. N Engl J Med. 1995;333(18):1184-9.

1. Kono A, Kanaya Y, Fujita T, Tsumura C, Kondo T, Kushiyama K, Rubenstein LZ. Effects of a preventive home visit program in ambulatory frail older people: a randomized controlled trial. $J$ Gerontol A Biol S Med Sci. 2012;67(3):302-9

12. Ploeg J, Brazil K, Hutchison B, Kaczorowski J, Dalby DM, Goldsmith CH, Furlong W. Effect of preventive primary care outreach on health related quality of life among older adults at risk of functional decline: randomised controlled trial. BMJ. 2010;340:c1480.

13. Bouman A, van Rossum E, Evers S, Ambergen T, Kempen G, Knipschild P. Effects on health care use and associated cost of a home visiting program for older people with poor health status: a randomized clinical trial in the Netherlands. J Gerontol A Biol Sci Med Sci. 2008;63(3):291-7.

14. Melis RJF, Adang E, Teerenstra S, van Eijken MIJ, Wimo A, van Achterberg T, van de Lisdonk EH, Rikkert MGM. Cost-effectiveness of a multidisciplinary intervention model for community-dwelling fral older people. J Gerontol A Biol Sci Med Sci. 2008;63(3):275-82.

15. Daniels R, Metzelthin $S$, Van Rossum E, De Witte L, Van den Heuvel W. Interventions to prevent disability in frail community-dwelling older persons: an overview. Eur J Ageing 2010;7(1):37-55

6. Daniels R, van Rossum E, Metzelthin S, Sipers W, Habets H, Hobma S, van den Heuvel W, de Witte L A disability prevention programme for community-dwelling frail older persons. Clin Rehabil. 2011;25(11):963-74.

17. Metzelthin SF, van Rossum E, de Witte LP, Hendriks MR, Kempen Gl. The reduction of disability in community-dwelling frail older people: design of a two-arm cluster randomized controlled trial. BMC Public Health. 2010;10:511
18. Metzelthin SF, Van Rossum E, De Witte LP, Ambergen T, Sipers W, Hobma S, Kempen GIJM Effectiveness of an interdisciplinary primary care approach to reduce disability in community-dwelling frail older people: a cluster randomised controlled trial. BMJ. 2013;347:f5264

19. Metzelthin SF, Daniels R, Van Rossum E, Cox K, Habets H, de Witte LP, Kempen GIJM. A nurse-led interdisciplinary care approach to prevent disability among community-dwelling frail older people: large-scale process evaluation. Int J Nurs Stud. 2013;50 (9):1184-96.

20. Steverink N Slaets JPJ Schuurmans $H$, van Lis M. Measuring frailty: development and testing of the Groningen Frailty Indicator (GFI). Gerontologist. 2001;41(1):236-7.

21. Derckx E. Eerste nurse practitioners met differentiatie huisartsenzorg. Tijdschr Verpleegkundigen 2006:3:26-30

22. Smeulders ESTF, van Haastregt JCM, Ambergen T, Uszko-Lencer NHKM, Janssen-Boyne JJJ, Gorgels APM, Stoffers HEJH, Lodewijks-van der Bolt CLB, van Eijk JTM, Kempen GIJM. Nurse-led self-management group programme for patients with congestive heart failure: randomized controlled self-management group programme
trial. J Adv Nurs. 2010;66(7):1487-99

23. Oostenbrink JB, Bouwmans CAM, Koopmanschap MA, Rutten FFH. Handleiding voor kosten onderzoek; methoden en standaard kostprijzen voor economische evaluaties in de gezondheidszor [Dutch manual for costing: methods and standard costs for economic evaluations in healthcare] Diemen, The Netherlands: College voor Zorgverzekeringen; 2010.

24. Kempen GIJM, Miedema I, Ormel J, Molenaar W. The assessment of disability with the Groningen Activity Restriction Scale. Conceptual framework and psychometric properties. Soc Sci Med 1996;43(11):1601-10

25. Drummond MF, Sculpher MJ, Thorrance GW, O'Brien BJ, Stoddard GL. Methods for the economic evaluation of health care programmes. 3rd edition ed. New York: Oxford University Press; 2005.

26. Doun P. Modeling valuations for EuroQol health states. Med Care. 1997:35(11):1095-108.

7. Thompson S. G... Barber JA. How should cost data in pragmatic randomised trials be analysed? BM 2000;320(7243):1197-200.

28. Hoeymans $\mathrm{N}$, Van LH, Westert GP. The health status of the Dutch population as assessed by the EQ-6D. Qual Life Res. 2005, 14:655-63.

La in economic evaluations: the Dutch EQ-5D tariff. Ned Tijdschr Geneeskd. 2005;149(28):1574-8.

30. Lacas A, Rockwood K. Frailty in primary care: a review of its conceptualization and implications for practice. BMC Med 2012;10(4):1-9.

31. Beswick AD, Rees K, Dieppe P, Ayis S, Gooberman-Hill R, Horwood J, Ebrahim S. Complex interventions to improve physical function and maintain independent living in elderly people: systematic review and meta-analysis. Lancet. 2008;371(9614):725-35.

2. Wilson JMG, Jungner G. Principles and Practice of Screening for Disease. Geneva: World Health Organization. 1968. 
Appendix 1 Estimated intervention costs for a sample of $n=200$ (based on process evaluation)

\begin{tabular}{|c|c|c|c|c|c|}
\hline & & & & $\begin{array}{l}\text { Total } \\
\text { costs }(€) \\
\mathrm{n}=200\end{array}$ & $\begin{array}{l}\text { Costs } \\
(€) \\
\text { per } \\
\text { patient }\end{array}$ \\
\hline Intervention materials & & & & $1,200.00$ & 6.00 \\
\hline Training activities & & & & $16,500.00$ & 82.50 \\
\hline \multirow[t]{2}{*}{ Postal screening } & & & & $8,100.00$ & 40.50 \\
\hline & $\begin{array}{l}\text { Price } \\
\text { (per } \\
\text { hour) }\end{array}$ & Minutes & $\begin{array}{l}\text { Number } \\
\text { of } \\
\text { patients }\end{array}$ & $\begin{array}{l}\text { Total } \\
\text { costs } \\
(€) \\
n=200\end{array}$ & $\begin{array}{l}\text { Costs } \\
(€) \\
\text { per } \\
\text { patient }\end{array}$ \\
\hline \multicolumn{6}{|l|}{ Assessment practice nurse } \\
\hline - Home visit practice nurse & 50.00 & 90 & 200 & $15,000.00$ & 75.00 \\
\hline - Home visit GP & 50.00 & 45 & 80 & $3,000.00$ & 15.00 \\
\hline - Home visit occupational therapist & 50.00 & 45 & 40 & $1,500.00$ & 7.50 \\
\hline - Home visit physiotherapist & 50.00 & 45 & 30 & $1,125.00$ & 5.63 \\
\hline - Home visit other professionals & 50.00 & 45 & 20 & 750.00 & 3.75 \\
\hline Total & & & & $21,375.00$ & 106.88 \\
\hline \multicolumn{6}{|l|}{ Preliminary treatment plan } \\
\hline - Practice nurse & 50.00 & 10 & 25 & 208.33 & 1.04 \\
\hline $\begin{array}{l}\text { - Duo meeting } \\
\text { (practice nurse and GP) }\end{array}$ & 100.00 & 15 & 120 & $3,000.00$ & 15.00 \\
\hline - Team meeting (all professionals) & 200.00 & 20 & 40 & $2,666.67$ & 13.33 \\
\hline Total & & & & $5,875.00$ & 29.37 \\
\hline \multicolumn{6}{|l|}{ Definitive treatment plan } \\
\hline - Home visit (preparation included) & 50.00 & 90 & 100 & $7,500.00$ & 37.50 \\
\hline \multicolumn{6}{|l|}{ Toolbox } \\
\hline - Meaningful activities & 50.00 & 600 & 30 & $15,000.00$ & 75.00 \\
\hline $\begin{array}{l}\text { - Adapting the environment, } \\
\text { activities or skills }\end{array}$ & 50.00 & 600 & 50 & $25,000.00$ & 125.00 \\
\hline $\begin{array}{l}\text { - Social network and social } \\
\text { activities }\end{array}$ & 50.00 & 300 & 30 & $7,500.00$ & 37.50 \\
\hline - Daily physical activity & 50.00 & 600 & 35 & $17,500.00$ & 87.50 \\
\hline - Stimulating health & 50.00 & 300 & 40 & $10,000.00$ & 50.00 \\
\hline Total & & & & $75,000.00$ & 375.00 \\
\hline
\end{tabular}

Appendix 1 Continued

\begin{tabular}{|l|l|l|r|r|r|}
\hline & $\begin{array}{l}\text { Price } \\
\text { (per } \\
\text { hour) }\end{array}$ & Minutes & $\begin{array}{l}\text { Number } \\
\text { of } \\
\text { patients }\end{array}$ & $\begin{array}{l}\text { Total } \\
\text { costs (€) } \\
n=200\end{array}$ & $\begin{array}{l}\text { Costs } \\
(€)\end{array}$ \\
\hline $\begin{array}{l}\text { per } \\
\text { patient }\end{array}$ \\
\hline Other interventions & 50.00 & 120 & 30 & $3,000.00$ & 15.00 \\
\hline Evaluation and follow-up & & & & & \\
\hline - Practice nurse & 50.00 & 30 & 100 & $2,500.00$ & 12.50 \\
\hline $\begin{array}{l}\text { - Duo meeting } \\
\text { (practice nurse and GP) }\end{array}$ & 100.00 & 15 & 35 & 875.00 & 4.38 \\
\hline - Team meeting (all professionals) & 200.00 & 20 & 55 & $3,666.67$ & 18.33 \\
\hline Total & & & & $7,041.67$ & 35.21 \\
\hline TOTAL & & & & $145,591.67$ & 727.96 \\
\hline
\end{tabular}




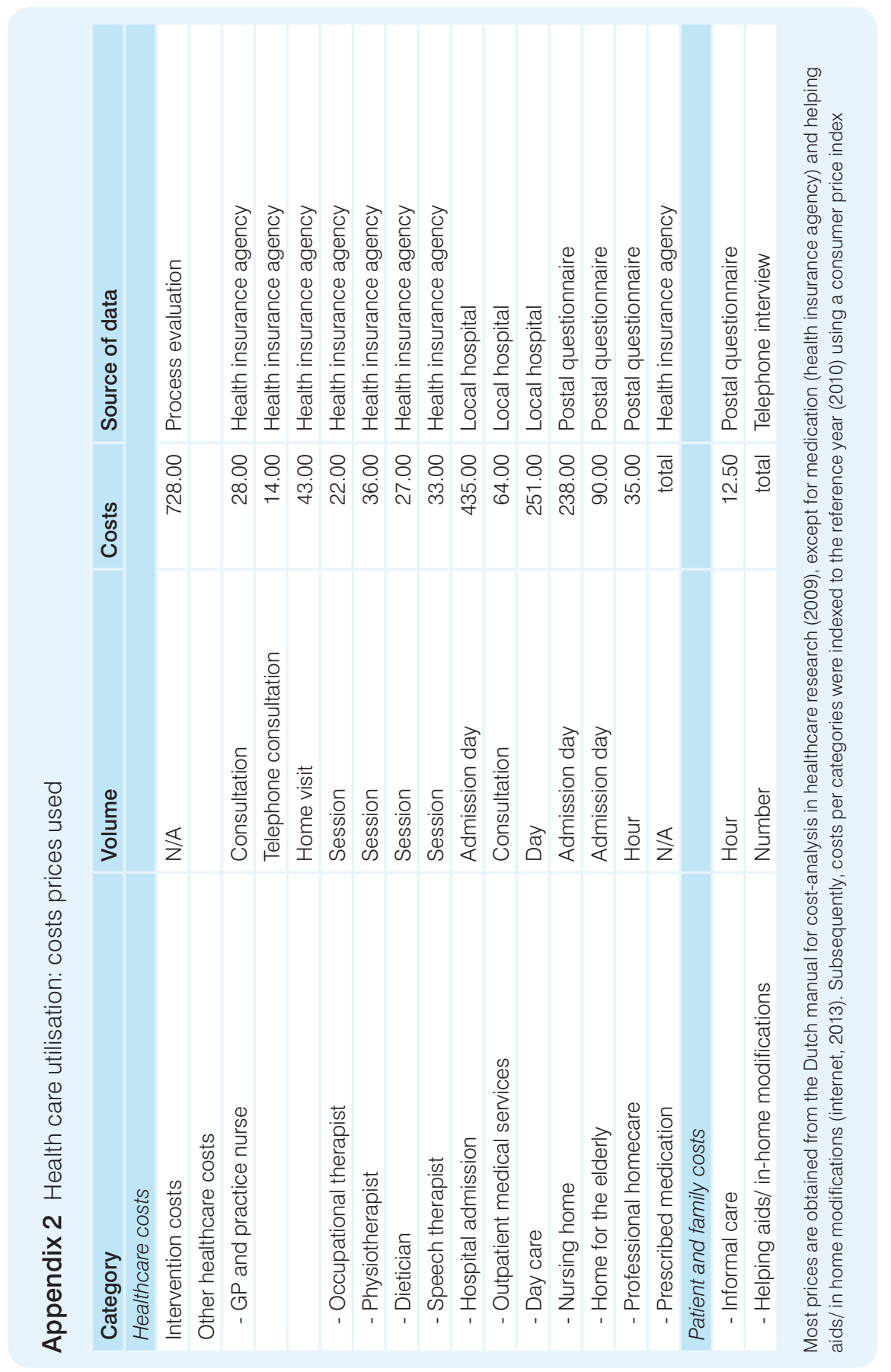

Appendix 3 Results of base-case and sensitivity analysis over 24 months $(n=194)$

\begin{tabular}{|l|l|l|l|l|l|}
\hline & ICER & \multicolumn{4}{|c|}{ Distribution of cost-effectiveness plane } \\
\hline & & NE\% & $\begin{array}{c}\text { NW\% } \\
\text { (inferior) }\end{array}$ & SW\% & $\begin{array}{c}\text { SE\% } \\
\text { (superior) }\end{array}$ \\
\hline Base-case analysis & & & & & \\
\hline QALYS UK & 150,616 & 0.19 & 0.77 & 0.02 & 0.02 \\
\hline GARS & 1,920 & 0.02 & 0.95 & 0.03 & 0.01 \\
\hline Sensitivity analyses & & & & & \\
\hline $\begin{array}{l}\text { Without intervention } \\
\text { costs }\end{array}$ & 132,195 & 0.18 & 0.76 & 0.03 & 0.04 \\
\hline QALYS NL & 285,428 & 0.29 & 0.68 & 0.02 & 0.02 \\
\hline
\end{tabular}

$\mathrm{NE}=$ north east quadrant (higher costs, more effects), $\mathrm{NW}=$ north west quadrant (higher costs, less effect), SW-south west quadrant (less costs, less effects), SE=south east quadrant (less costs, more ects), QALY=qually adjusted ilie years 


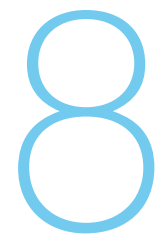

General Discussion

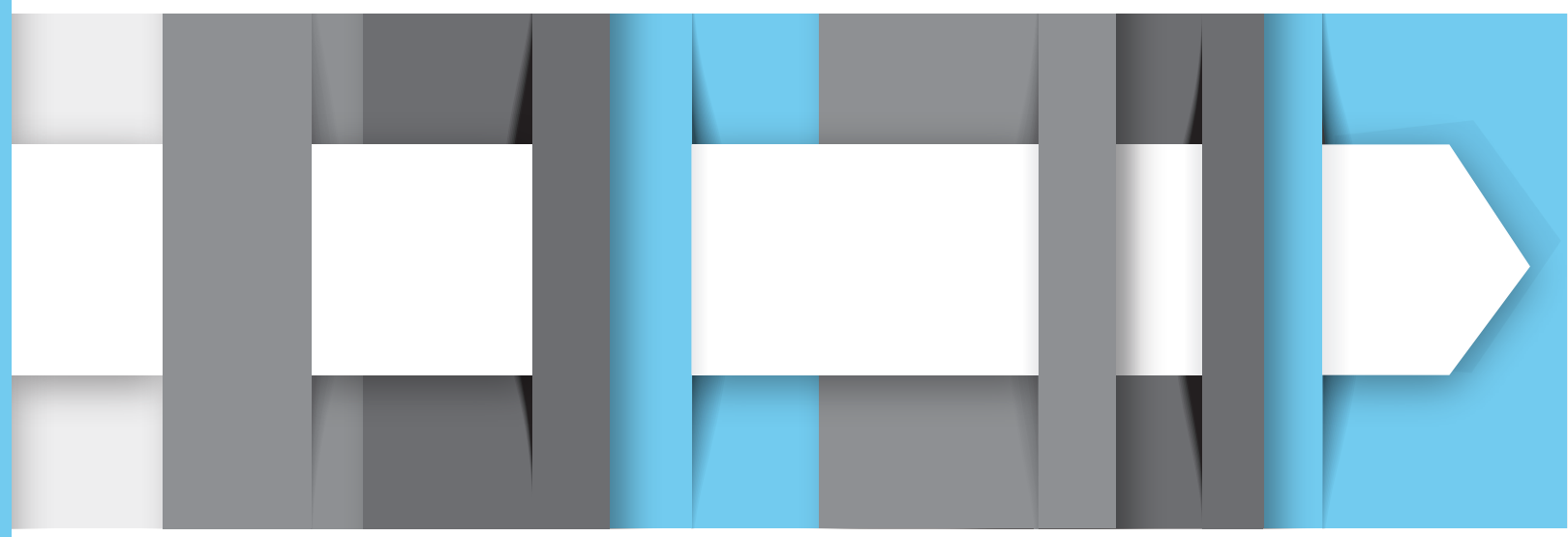




\section{Introduction}

The main objective of this thesis was to evaluate an interdisciplinary primary care approach for community-dwelling frail older people: the 'Prevention of Care' (PoC) approach. The aim of this approach is to reduce disability and prevent (further) functional decline. The PoC approach consists of a postal frailty screening, a multidimensional in-home assessment, interdisciplinary care based on a tailor-made treatment plan and regular evaluation and follow-up.

A cluster randomised trial was conducted to investigate the effectiveness and costeffectiveness of the PoC approach. Alongside the trial, a comprehensive process evaluation was done. In addition, a validation study was conducted evaluating and comparing three screening instruments for identifying frail older people in the community.

This final chapter summarises the main findings of this thesis. Furthermore, it discusses some methodological issues and reflects on theoretical considerations regarding the interpretation of the results. The chapter ends with implications for practice and future research.

\section{Main findings}

First, a validation study was conducted to evaluate and compare three frailty screening instruments: the Groningen Frailty Indicator (GFI), ${ }^{1}$ the Tilburg Frailty Indicator (TFI) ${ }^{2}$ and the Sherbrook Postal Questionnaire (SPQ). ${ }^{3}$ The GFI and the TFI showed high internal consistency and construct validity in contrast to the SPQ. Based on our findings it is not yet possible to conclude whether the GFI or the TFI should be preferred, but the SPQ seems less appropriate.

Second, a comprehensive process evaluation was conducted in the intervention group to provide insight into the extent to which the PoC approach was implemented as intended and to evaluate experiences of healthcare professionals and frail older people regarding benefits, burden, stimulating factors and barriers. Data was collected from 193 older people and 45 healthcare professionals. Although some parts of the protocol were insufficiently executed, healthcare professionals and frail older people were satisfied with the PoC approach; it provided a useful structure for the delivery of geriatric primary care and increased the attention to preventive treatment. Frail older people felt acknowledged by healthcare professionals and experienced support in handling their problems and fulfilling their wishes. Given its complexity, the implementation of the PoC approach was challenging and some parts of the protocol need further attention.

Third, to test the effectiveness of the PoC approach a cluster randomised controlled trial was conducted among twelve general practitioner practices, which 
were randomly allocated to the intervention (six practices - PoC approach) or control group (six practices - usual care). Based on a postal questionnaire, including the GFI, 346 frail older people were included in the study. Their mean age was 77.2 years and $58 \%$ were female. Patient follow-up rates were $91 \%$ at 6 months, $86 \%$ at 12 months and $78 \%$ at 24 months with significantly more loss to follow-up in the intervention group. Mixed-model multi-level analyses showed no significan differences between the two groups with regard to all primary (disability) and secondary outcomes (i.e., depressive symptomatology, social support interactions, fear of falling and social participation, quality of life) at 6, 12 and 24 months follow-up. Pre-planned subgroup analyses confirmed these results.

Finally, embedded in the trial a cost-effectiveness (CEA) and cost-utility analysis (CUA) was performed from a societal perspective with a time horizon of 24 months. People in the intervention group had significantly more contacts with the practice nurse $(5.2$ versus $1.1, p<0.001)$ and occupational therapy sessions $(7.1$ versus 1.2 $p<0.001)$ over a period of two years than people in the control group. Remaining categories of healthcare utilisation were comparable between the groups. Total healthcare costs in the sample of complete cases (56\%) were 26,503 in the intervention group compared to 20,550 in the control group. Despite higher healthcare costs in the intervention group we found no significant differences between the groups with regard to disability and quality of life.

\section{Methodological considerations}

Regarding the methodological strengths and limitations of our trial the following issues will be discussed: 1) study design, 2) recruitment of participants, 3) outcome measures, and 4) treatment fidelity

\section{Study design}

Randomised controlled trials are considered to be a gold standard for evaluating interventions, as they estimate the impact of an intervention through direct comparison with a randomly allocated control group. Randomisation is the best way to ensure that factors, which may affect the outcome of an intervention, are evenly distributed among study groups. ${ }^{4}$ In the current study individual randomisation was inappropriate it was assumed that organisational circumstances and professional behaviour affect the treatment. Consequently, a cluster design was chosen to avoid contamination bias between the groups. Unfortunately, this choice led to significant baseline differences between the groups with regard to frailty and disability and a skewed sample size distribution. These are common risks in a cluster approach. ${ }^{4}$ Anothe problem in cluster randomised designs is within-cluster correlation leading to a greater homogeneity of individuals within a cluster, which results in a loss of power for detecting differences between the groups. ${ }^{5}$ In order to correct for the disadvantages of a cluster approach, an Intra Cluster Correlation (ICC) coefficient was taken into account in the sample size calculation and outcome estimates of the multileve analyses were adjusted for significant differences at baseline by including these two variables as covariates in each model. Despite all efforts taken, the methodological drawbacks of a cluster randomised design may have affected our findings to some extent.

Recruitment of participants

One of the biggest challenges in this study was the recruitment of appropriate participants. During the last decades, the concept of frailty has been established to describe older people with an increased risk for adverse outcomes. However, there is still a lively debate about the definition and measurement of frailty. ${ }^{6}$ In the curren study, postal screening (GFI) followed by a comprehensive assessment, has shown to be a feasible method to identify community-dwelling frail older people. However there are still some doubts about the psychometric properties of the chosen screening instrument. The GFI showed high internal consistency and construct validity in a cross-sectional validation study, which is part of this thesis (Chapter 2), but information about the predictive validity was lacking when the trial started. Two recent validation studies ${ }^{7,8}$ have shown that the GFI needs further improvement regarding its accuracy. There are numerous other self-report instruments available, but all of them are in the early stage of development and their psychometric properties are still insufficient or unclear. ${ }^{7,9}$

An alternative for self-report instruments are performance-based measures. It is assumed that performance-based measures provide more precise and valid answers as they are less influenced by socio-demographic variables, personality and cognitive and affective factors. However, they are more sensitive to non-response, changes in time and differences in the execution of activities. Furthermore, they are less easy to conduct and time-consuming. 10-12 When considering performance-based measures modern technologies have the potential to monitor frailty markers efficiently. For example, a modified bathroom scale, a grip ball, or a mobile phone with built-in accelerometer are relatively inexpensive technologies to measure balance, grip strength and physical activity, which are known as relevant predictors for adverse health outcomes in older people.13,14 In a recent validation study, the modified bathroom scale was compared with clinical balance tests and provided promising results. However, more research into their validity, feasibility and clinical relevance is needed. ${ }^{15}$ 
Outcome measures

This study, like many intervention studies in this domain, is based primarily on self-report measures. To increase the validity of the outcome measurements severa strategies were applied. First, assessed by blinded data collectors, outcome measures with good psychometric properties were used. Second, missing values were minimised by using telephone interviews for most outcome measures. To complete missing data gathered via postal questionnaires, participants were contacted by telephone. This method led to relatively few missing data and high follow-up rates. Third, during the economic evaluation, registries from health insurance agencies and the local hospital were used as primary sources for data collection. Only data that could not be obtained from the registries were collected via self-report.

Despite a thoughtful selection of outcome measures and data collection methods, we failed to include some measures of relevant intermediate outcomes that are strongly related to the PoC approach, for example, the achievement of individual goals. Older people interviewed during the process evaluation (Chapter 5) felt acknowledged by healthcare professionals and experienced support in handling their problems and fulfilling their wishes. However, our outcome measures, which were focussed on frail older peoples' health status rather than on the achievement of their goals, did not detect any effects. A study by Rockwood and colleagues evaluating a comprehensive geriatric assessment and management approach has shown that Goal Attainment Scaling ${ }^{16}$ is more responsive to clinical relevant changes than for example (I)ADL measures. ${ }^{17}$ We probably missed clinically important changes due to our choice of outcome measures. Furthermore, additional information about intermediate outcomes would have been useful to explain the lack of effects.

Treatment fidelity

Being aware of the complexity of the PoC approach, much effort was put into a comprehensive process evaluation (Chapter 5). A mixed methods design was applied, integrating quantitative and qualitative data from different sources. Nevertheless, to some extent it remains unclear what has happened in daily practice. While healthcare professionals involved were very positive about the implementation of the PoC approach during the semi-structured and focus group interviews, the logbooks showed that not all the steps of the PoC approach were applied. This finding is in line with previous studies in this field ${ }^{18,19}$ which have shown that healthcare professionals have difficulties in reflecting adequately on their functioning. perhaps due to a lack of awareness of their own behaviour. These researchers suggest more objective measures like video- or audio-taping to increase treatment fidelity. It is also insufficiently known what happened in the control group, as no fidelity data were collected in this group. Based on the economic evaluation (Chapter 7) it is known that older people in the control group had comparable volumes of healthcare utilisation part from contacts with practice nurses and occupational therapists, which were more frequent in the intervention group. Older people in the control group probably received active ingredients of the PoC approach as well resulting in insufficien contrast between the groups and consequently a reduced effect size. Detailed treatment fidelity data measured in both groups would have been useful to provide more insight into the differences between the groups.

\section{Theoretical considerations}

This section provides some theoretical considerations regarding the interpretation of the results. The following issues will be discussed: 1) target group, 2) ingredients of the PoC approach, and 3) the implementation of the PoC approach.

Target group

Earlier in this chapter we reflected on some psychometric issues regarding the recruitment of frail older people. However, there are also some theoretica considerations regarding the target group of the PoC approach. The PoC approach is based on a multifactorial perspective on frailty. ${ }^{20,21}$ Compared to the frailty phenotype ${ }^{22,23}$ this perspective leads to a more heterogeneous target group with a great diversity in deficits (i.e., physical, psychological and social). Also the severity of health problems varied, as the PoC approach focuses on both older people in a relatively early stage of frailty with no disability and older people who are already disabled. Obviously, the heterogeneity of the target group has consequences for healthcare delivery. Frail older people who are already disabled may benefit from a rehabilitative approach that attempts to improve functional status. In contrast, older people with no disability may profit, to a larger extent, from a health promotion and prevention approach that attempts to reduce risk factors for functional decline. Furthermore, physically frail older people may need another approach than older people with psychological or social problems. Consequently, the PoC approach is designed in a tailor-made way and the guidelines for assessment and treatment cover the full range of deficits irrespective of their severity. However, the process evaluation has shown that practice nurses and GPs in our study were used to focus particularly on specific diseases and existing disability; they paid less attention to psycho-social problems and the enhancement of meaningful activities. Older people with hardly any disability in terms of activities of daily living were in their view not (yet) frail and consequently not eligible for the PoC approach. A preventive approach and a multifactorial perspective on frailty may therefore not fit very well with their (current) philosophy of healthcare delivery. 
In an effort to prevent (further) functional decline, it is still not clear which older people would benefit the most from interventions like the PoC approach. Several previous reviews in the field of preventive home visiting programmes suggested that interventions may be more effective in low-risk, non-disabled older people. ${ }^{24,25}$ This is in line with a more recent review reporting that frail older people have to be identified in a relatively early phase when negative health outcomes can still be avoided. ${ }^{26}$ Participants in our study were probably, at least in part, too frail. The baseline disability score in our sample was substantially higher compared to the Dutch general population of those 70 years of age and older. ${ }^{8}$ Still, subgroup analyses based on the baseline scores of frailty (pre-planned) and disability (post-hoc) did not reveal other results.

Ingredients of the PoC approach

With regard to community-based care for frail older people, Dutch policy makers recommend pro-active, client-centred care. ${ }^{27}$ Due to the complex healthcare needs of frail older people, care should be delivered by an interdisciplinary team coordinated by a case-manager to ensure integrated care. ${ }^{27}$ The importance of these components is supported by the review in this thesis (Chapter 3). In addition, we suggested selfmanagement support and engagement in meaningful activities as promising elements for the reduction of disability and prevention of (further) functional decline. It was expected that combining these ingredients would lead to an intervention that is more (cost-) effective than usual care. However, no significant results were found. Due to the complexity of the PoC approach it is difficult to interpret these unexpected results. According to the Medical Research Council (MRC) ${ }^{28,29}$ complex interventions 1) have a high number of interacting components, 2) affect behaviour of those delivering and receiving care, 3) target a variety of groups, 4) affect variable outcomes, and 5) need a degree of flexibility and tailoring during implementation. Consequently, active ingredients of the PoC approach could have been limited in their effectiveness by other components. Perhaps the right ingredients were chosen, but the combination led to an approach that was too complex to apply in daily practice resulting in insufficient delivery or receipt by professionals and older people. In addition, it is unclear whether the components of the PoC approach are sufficiently tailored to the needs and preferences of the heterogeneous target group of frail older people. In conclusion, it is not possible to determine whether or not the right ingredients were chosen for the PoC approach and which (combination of) ingredients work better than others.

Implementation of the PoC approach

Although healthcare professionals in this study appreciated the PoC approach, the process evaluation (Chapter 5) has shown that some parts of the protocol were insufficiently executed. For example, 1) fewer older people than expected were referred to other disciplines; 2) the problem analysis and the development of treatment plan was often not done within the team of professionals involved; 3) half of the frail older people did not receive a second home visit to discuss their treatment plan or follow-up activities; 4) the toolbox, including guidelines for assessment and treatment and the theoretical models to stimulate behavioural change and self-management, were not frequently applied; 5) the extent of evaluation and follow-up was limited; and 6) healthcare professionals focussed too much on risk factors for disability or existing problems in performing daily activities instead of enhancing meaningful activities. The interviews of the process evaluation provided some reasons for the insufficient implementation of the PoC approach. Professionals mentioned that some parts of the intervention protocol were (too) time-consuming or difficult to apply. They expressed a need for more on the job training and more opportunities to exchange experiences with each other. Despite an extensive development period and a comprehensive training program, we probably failed in providing professionals with the needed competencies, feasible tools or environmenta circumstances to apply the PoC approach in daily practice. For example, the development of individualised goals, a prerequisite for tailor-made care and selfmanagement support, is a challenging task, as patients tend to adopt a passive role in goal setting. ${ }^{30}$ Tools to facilitate goal-setting might have been useful to add. Furthermore, healthcare professionals are used to focusing their treatment on single diagnosis. ${ }^{6}$ Maybe professionals lack the competencies to deal with multiple interacting medical and social problems, which characterise frail older people. In addition, the structure of a predominantly reactive healthcare system in the Netherlands ${ }^{31}$ may have worked against a proactive approach and the philosophy of healthcare delivery has to be changed first.

\section{Implications}

This thesis results in several implications for practice and future research.

Practice

First, frail older people are a heterogeneous target group with a great diversity in deficits (i.e., physical, social and psychological), risks and severity of health problems Interdisciplinary collaboration, client-centeredness, self-management support, engagement in meaningful activities and case management are suggested as relevant ingredients in the scientific literature and recent policy reports to increase the quality of care for frail older people. These recommendations have led to the development of highly complex interventions. However, we have to critically consider 
how much complexity healthcare professionals can handle and how to design interventions that are as simple and straightforward as possible.

Second, although an intensive training program was offered, additional time and efforts seem to be necessary to change well-established patterns of care. An initial educational program, review of specific competencies needed and on-going supervision and encouragement to implement new activities are recommended to facilitate the implementation of complex interventions. In addition, changing behaviou requires a comprehensive approach at different levels such as patient, professionals, and environment. ${ }^{32}$ Taking into account a socio-ecological model may contribute to the understanding of intrapersonal, interpersonal, environmental and policy factors and may facilitate changes in health behaviour, current care philosophies and care practices. ${ }^{33}$ Consequently, successful interventions have to focus on both patients and professionals, while taking into account their environmental circumstances

Lastly, although no difference in effects between the intervention and contro group was found, implementation of the PoC approach results in higher healthcare costs compared to usual care. Consequently, the implementation of the PoC approach, in its current form, is not recommended, at least not in the Netherlands or in other countries with a similar healthcare system. In the Netherlands, standard healthcare is already at a relatively high level. Nearly all people are covered by healthcare insurance, care is easily accessible and its quality is often considered to be good. ${ }^{34}$ Consequently, it is difficult to add something to increase the (cost-) effectiveness of current healthcare delivery. Within the Dutch National Care for the Elderly Program, several other projects have focused on community-based care in frail older people as well. ${ }^{35-38}$ Despite some variances, the projects have several features in common like screening and assessment, tailor-made treatment, integrated healthcare delivery, and regular monitoring and follow-up. It is not yet clear, whether these projects will provide significant results, but it seems obvious that findings have to be combined in a next step to make final recommendations on how to reduce disability and prevent (further) functional decline in community-dwelling frail older people.

Research

First, this thesis has shown that more research into the conceptualisation and measurement of frailty is needed. On the one hand it is not known which older people have to be considered as frail and would benefit the most from which intervention. On the other hand, the validity of available screening methods to measure frailty has to be improved. Given the large population which were screened, a two-step approach consisting of a short postal screening followed by a comprehensive in-home assessment has shown to be feasible. However, it is not yet clear which screening instruments (first step) are preferred in identifying frail older people for the second step. Available screening instruments that could be used for the postal screening have shown not to be sensitive enough for the purpose of screening and diagnosis.7,8,26 They can be used for the exclusion of non-frail older people rather than for casefindings. ${ }^{26}$ An alternative for postal screenings are performance-based measures For example, modern technologies can be used to measure the performance of older people on a regular basis; this fits the dynamic nature of frailty. ${ }^{39}$ Moreover, the combination of self-reported and performace-based measures is also promising, but research in this area is just in the initial stage.

Second, despite a comprehensive process evaluation, which was conducted alongside the trial, it remains questionable whether or not the lack of effects in this study is due to an ineffective intervention or insufficient implementation of the PoC approach. Furthermore, it is not known whether older people in the control group received active ingredients of the $\mathrm{PoC}$ approach resulting in insufficient contrasts between the groups and consequently a reduced effect size. It is recommended that in the future more time and efforts is focused on the measurement of treatment fidelity (e.g., by using audio-/ videotaping), in both the intervention and control group to draw accurate conclusions about treatment effectiveness ${ }^{40}$

Finally, it has to be considered whether or not randomised controlled trials are the most appropriate design to evaluate complex interventions like the PoC approach in which individual randomisation is inappropriate. One variant of the cluster tria design is the stepped wedge trial design, which is in essence a one-way crossove cluster trial. The stepped wedge trial design requires fewer clusters and maximises statistical power compared with a parallel-group cluster randomised trial design; the intervention effect is estimated not only by between-cluster comparisons but also by within-cluster comparisons. Also, the phased implementation allows for improvement of the intervention or its delivery where necessary before the next implementation phase. ${ }^{41}$ It is recommended that in the future when planning a study on complex interventions, these innovative design techniques are considered. 


\section{References}

1. Steverink N, Slaets JPJ, Schuurmans H, van Lis M. Measuring frailty: development and testing of the Groningen Frailty Indicator (GFI). Gerontologist. 2001;41(1):236-7.

2. Gobbens RJJ, van Assen MALM, Luijkx KG, Wijnen-Sponselee MT, Schols JMGA. The Tilburg Frailty Indicator: psychometric properties. J Am Med Dir Assoc. 2010;11(5):344-55.

3. Hebert R, Bravo G, Korner-Bitensky N, Voyer L. Predictive validity of a postal questionnaire for 996:25(2):159-67.

4ccles M, Grimshaw J, Campbell M, Ramsay C. Research designs for studies evaluating the effectiveness of change and improvement strategies. Qual Saf Health Care. 2003;12:47-52.

5. Parker DR, Evangelou E, Eaton CB. Intraclass correlation coefficients for cluster randomized trials in primary care: the cholesterol education and research trial (CEART). Contemp Clin Trials.

6. Lacas A, Rockwood K. Frailty in primary care: a review of its conceptualization and implications for practice. BMC Med 2012;10(4):1-9.

7. Hoogendijk EO, van der Horst HE, Deeg DJ, Frijters DH, Prins BA, Jansen AP, Nijpels G, van Hout HP. The identification of frail older adults in primary care: comparing the accuracy of five simple instruments. Age Ageing. 2013;42(2):262-5.

8. Daniels R, Van Rossum HIJ, Beurskens A, Van den Heuvel W, De Witte L. The predictive validity of three self-report screening instruments for identifying frail older people in the community. BMC Public

Health. 2012;12:69.
9. De Vries NM, Staal JB, van Ravensberg CD, Hobbelen JS, Olde Rikkert MG, Nijhuis-van der Sanden MW. Outcome instruments to measure frailty: a systematic review. Ageing Res Rev. 2011;10(1):104-14

. Guralnik JM, Branch LG, Cumm Gerontol. 1989;44(5):M141-6.

11. Kempen GIJM, van Heuvelen MJG, van den Brink RHS, Kooijman AC, Klein M, Houx PJ, Ormel Factors affecting contrasting results between self-reported and performance-based levels of physica limitation. Age Ageing. 1996;25(6):458-64

12. Martin FC, Brighton P. Frailty: different tools for different purposes? Age Ageing. 2008;37(2):129-31.

3. Vermeulen J, Spreeuwenberg MD, Daniels R, Neyens JC, Van Rossum E, De Witte LP. Does a falling level of activity predict disability development in community-dwelling elderly people? Clin Rehab 2013;27(6):546-54

14. Vermeulen J, Neyens JC, van Rossum E, Spreeuwenberg MD, de Witte LP. Predicting ADL disability in community-dwelling elderly people using physical frailty indicators: a systematic review. BMC Geriat. 2011;11:33

15. Vermeulen J, Neyens JC, Spreeuwenberg MD, van Rossum E, Hewson DJ, Duchêne J, de Witte LP. Construct validity of a modified bathroom scale that can measure balance in elderly people. J Am Med Dir Assoc. 2012;13(7):665.e1-5

16. Kiresuk TJ, Sherman RE. Goal Attainment Scaling: a general method for evaluating comprehensive community mental health programs. Community Ment Health. 1968; 1(4):443-53

17. Rockwood K, Howlett S, Stadnyk K, Carver D, Powell C, Stolee P. Responsiveness of goal attainment scaling in a randomized controlled trial of comprehensive geriatric assessment. J Clin Epidemio. 2003:56:736-43.

18. Dorresteijn TAC, Zijlstra GAR, van Haastregt JCM, van Vlaeyen JWS, Kempen GIJM. Feasibility of a nurse-led in-home cognitive behavioral program to manage concerns about falls in frail older people: a process evaluation. Res Nurs Health. 2013;36(3):257-70.

19. Heinrich E. Diabetes self-management; strategies to support patients and healthcare professionals [PhD Thesis]. Maastricht: Health Medicine and Life Sciences, Maastricht University; 2012.

20. Bergman H, Beland F, Karunanthan S, Hummel S, Hogan D, Wolfson C. Developing a working framework for understanding frailty. (English translation of article published in) Gerontol Soc. 2004; 109:15-29.
21. Gobbens RJ, van Assen MA, Luijkx KG, Schols JM. Testing an integral conceptual model of frailty. J Adv Nurs. 2012;68(9):2047-60.

22. Fried LP, Ferrucci L, Darer J, Williamson JD, Anderson G. Untangling the concepts of disability frailty, and comorbidity: implications for improved targeting and care. J Gerontol A Biol Sci Med Soi 2004;59(3):255-63

23. Fried LP, Tangen CM, Walston J, Newman AB, Hirsch C, Gottdiener J, Seeman T, Tracy R, Kop WJ, Burke G McBurnie MA. Frailty in older adults: evidence for a phenotype $J$ Gerontol A Biol Sci Med Sci 2001;56(3):M146-56

24. Stuck AE, Egger M, Hammer A, Minder CE, Beck JC. Home visits to prevent nursing home admission and functional decline in elderly people: systematic review and meta-regression analysis. JAMA. 2002;287(8):1022-8.

25. Stuck AE, Walthert JM, Nikolaus T. Bula CJ, Hohmann C, Beck JC. Risk factors for functional status decline in community-living elderly people: a systematic literature review. Soc Sci Med. 1999;48(4):445

26. Piipers E, Ferreira I, Stehouwer CDA, Nieuwenhuijzen Kruseman AC. The frailty dilemma. Review of the predictive accuracy of major frailty scores. Eur J Intern Med. 2012;23(2):118-23.

27. RVZ. Redzaam ouder. Zorg voor niet-redzame ouderen vraagt om voorzorg door iedereen. The Hague The Netherlands: Raad voor de Volksgezondheid \& Zorg;2012.

28. Craig P, Dieppe P, Macintyre S, Michie S, Nazareth I, Petticrew M. Developing and evaluating complex interventions: the new Medical Research Council guidance. BMJ. 2008;29:337:a1655.

29. Medical Research Council. A framework for the development and evaluation of RCTs for complex interventions to improve health. London: MRC; 2000

30. Siegert RJ, Taylor WJ. Theoretical aspects of goal-setting and motivation in rehabilitation. Disabil Rehabil. 2004;26(1):1-8.

31. Synthesis report on the public consultation on the European Innovation Partnership on Active and Healthy Ageing. Retrieved from http://ec.europa.eu/research/innovation-union/index_en.cfm?section=active-healthy-ageing\&pg=consultation. Accessed June 2012.

32. Grol R, Grimshaw J. From best evidence to best practice: effective implementation of change in patients' care Lancet. 2003:362(9391):1225-30

33. Resnick B. Changing the philosophy of care - a function-focused care approach. Aging Well. 2012;5(2):34.

34. Smeulders ESTF, van Haastregt JCM, Ambergen T, Uszko-Lencer NHKM, Janssen-Boyne JJJ, Gorgels APM, Stoffers HEJH, Lodewijks-van der Bolt CLB van Eijk JTM, Kempen GIJM. Nurse-led self-management group programme for patients with congestive heart failure: rand omized controlled trial. J Adv Nurs. 2010:66(7):1487-99.

35. Bleijenberg N, Drubbel I, ten Dam VH, Numans ME, Schuurmans MJ, de Wit NJ. Proactive and integrated primary care for frail older people: Design and methodological challenges of the Utrech Primary care PROactive Frailty Intervention Trial (U-PROFIT). BMC Geriatr. 2012;12(16).

36. Muntinga ME, Hoogendijk EO, van Leeuwen KM, van Hout HPJ, Twisk JWR, van der Horst HE, Nijpels G, Jansen APD. Implementing the chronic care model for frail older adults in the Netherlands: study protocol of ACT (frall older adults: care in tranisition). BMC Geriatr. 2012;12(19):1-10.

37. Suijker JJ, Burrman BM, ter Riet G, van Rijn M, de Haan RJ, de Rooij SE, Moll van Charante EP. Comprehensive geriatric assessment, multifactorial interventions and nurse-led coordination to prevent functional decline ammong community-dwelling older persons: protocol of a cluster randomized trial. BMC Health Serv Res. 2012;12(85):1-12.

38. Stijnen MMN, Duimel-Peeters IGP, Jansen MWJ, Vrijhoef HJM. Early detection of health problems in potentially frail community-dwelling older people by general practices - project [G]OLD: design of a Iongitudinal, quasi-experimental study. BMC Geriatr. 2013;13(7):1-10.

39. Studenski S, Hayes RP, Leibowitz RQ, Bode R, Lavery L, Walston J, Duncan P, Perera S. Clinical globa impression of change in physical frailty: development of a measure based on clinical judgment. J Am Geriatr Soc. 2004;52(9):1560-6. 
40. Bellg AJ, Borrelli B, Resnick B, Hecht J, Minicucci DS, Ory. M., Ogedegbe G, Orwig. D., Ernst D, Czajkowski S, Treatment Fidelity Workgroup of the $\mathrm{NIH}$ Behavior Change Consortium. Enhancing
treatment fidelity in health behavior change studies: best practices and recommendations from the NIH Behavior Change Consortium. Health Psychol. 2004;23(5):443-51.

41. Mdege ND, Man MS, Taylor nB, C.A., , Torgerson DJ. Systematic review of stepped wedge cluster randomized trials shows that design is particularly used to evaluate interventions during routine implementation. J Clin Epidemiol. 2011;64(9):936-48. 


\section{Summary}

Nederlandse samenvatting

Deutsche Zusammenfassung

Dankwoord | Dankwort

About the author

List of publications

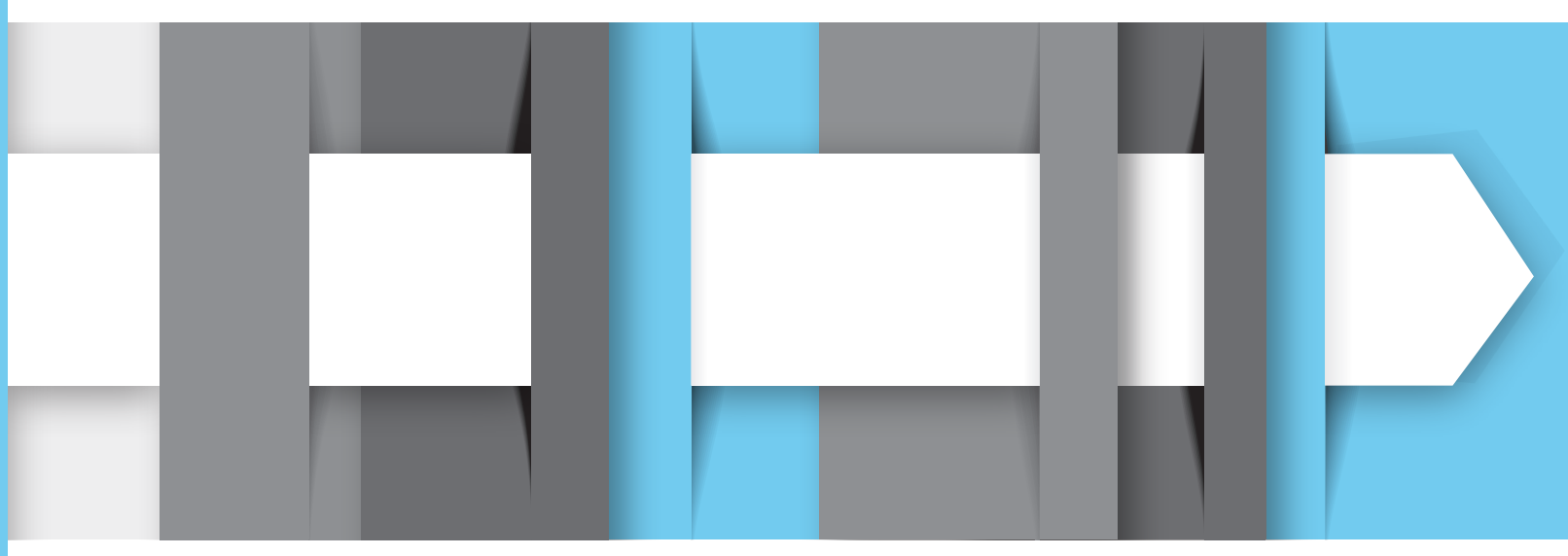




\section{Summary}

Evaluation of the Prevention of Care Approach

This thesis reports on the evaluation of an interdisciplinary primary care approach for community-dwelling frail older people: the 'Prevention of Care' (PoC) approach. The aim of this approach is to reduce disability and prevent (further) functional decline The PoC approach consists of six steps: (1) first a postal screening for frailty takes place using the Groningen Frailty Indicator (GFI); (2) identified frail older people receive a comprehensive geriatric assessment during a home visit by the practice nurse and if indicated additional assessments by other professionals (e.g. occupational therapist or a geriatrician); (3) after the assessment a preliminary treatment plan is formulated, either in a bilateral meeting (general practitioner and practice nurse) or in an extended team meeting consisting of a general practitioner, practice nurse, occupational and physiotherapist and, if indicated, other healthcare professionals; (4) during a second home visit by the practice nurse a final treatment plan is formulated, including a list of goals, strategies and actions that meet the older person's needs; (5) subsequently, the treatment starts (step 5). The intervention protocol offers a toolbox with recommendations and guidelines for the execution of the treatment plan; (6) finally, the achievement of goals and the implementation of strategies in daily life is evaluated and appointments for follow-up are made (see Appendix for a more detailed description of the approach).

Chapter 1 provides information about the concept of 'frailty' and 'disability' in older people. Furthermore, the relevance of community-based care for frail older people is introduced and recent developments in the Dutch healthcare system are described. The chapter ends with the main objectives of this thesis.

To reduce disability and prevent (further) functional decline frail older people have to be identified in time. Consequently, valid screening instruments are needed. However the empirical evidence with regard to the psychometric properties of available instruments is scarce. A validation study (Chapter 2) was conducted to evaluate and compare three self-report screening instruments: the 'Groningen Frailty Indicator' (GFI), the 'Tilburg Frailty Indicator' (TFI) and the 'Sherbrook Postal Questionnaire' (SPQ). The GFI and the TFI showed high internal consistency and construct validity in contrast to the SPQ. Based on our findings it is not yet possible to conclude whether the GFI or the TFI should be preferred, but the SPQ seems less appropriate.

During the last decades many intervention studies targeting community-dwelling frail older people have been conducted. Although several meta-analyses and systematic 
reviews were conducted focusing on specific categories of interventions, mostly in the field of preventive home visiting programmes and physical exercises, no review was available that provides an overview of the full range of existing interventions aimed at community-dwelling frail older people with 'disability' as an outcome measure. Consequently, a narrative review (Chapter 3) was conducted to identify and summarise promising components for future intervention programmes that aim to reduce disability and prevent (further) functional decline. Based on the review it is suggested that community-based interventions for frail older people should be conducted by an interdisciplinary primary care team, involving (population) screening, individualised assessments and interventions (tailor-made care), self-management support, engagement in meaningful activities, case management and long-term follow-up. Promising elements identified in the narrative review were combined in the PoC approach

To evaluate its feasibility, effectiveness and cost-effectiveness a cluster randomised controlled trial was conducted among twelve general practitioner practices, which were randomly allocated to the intervention group (six practices - PoC approach) or control group (six practices - usual care) (Chapter 4). A postal questionnaire, including the GFI, was send to 3,498 of their patients ( $\geq 70$ years) to identify frail older people. The response rate was $80 \%$. Of the older people who were willing to participate, $36 \%$ was frail according to the GFI scores (GFI $\geq 5$ ). Finally, 346 frail older people were included in the trial, which consists of three interrelated sub-studies: a process evaluation, an effect evaluation and an economic evaluation

First, a comprehensive process evaluation (Chapter 5) was conducted using a mixed methods design to provide insight into the extent to which the PoC approach was implemented as intended and to evaluate experiences of healthcare professionals and frail older people regarding benefits, burden, stimulating factors and barriers. Data were collected from participating older people and healthcare professionals. Professionals were satisfied with the PoC approach, as it provided a useful structure for the delivery of geriatric primary care and increased the attention to interdisciplinary cooperation and preventive treatment. Frail older people felt acknowledged by healthcare professionals and experienced support in handling their problems and fulfilling their wishes. Given its complexity, some parts of the intervention protoco were not implemented as planned. The problem analysis and the development of a preliminary treatment plan (step 3) was often not done in a bilateral or an extended team meeting and only half of the treatment plans were discussed with the frail olde person (step 4). Also, the toolbox parts were not frequently used in the treatment phase (step 5), and the extent of evaluation and follow-up was limited (step 6).
Second, with regard to disability (primary outcome) and several secondary outcomes, the effectiveness of the PoC approach was evaluated (Chapter 6 ). In the intervention group (6 practices), 193 frail older people received the PoC approach compared to 153 in the control group (6 practices) who received care as usual. The mean age of the total sample was 77.2 years; $58 \%$ of the sample was female, $49 \%$ was living alone and $58 \%$ had a low level of education. At baseline, participants in the intervention group were significantly frailer and more disabled than those in the control group. Patient follow-up rates were $91 \%$ at 6 months, $86 \%$ at 12 months and $78 \%$ at 24 months, with significantly more loss to follow-up in the intervention group. Data on our primary and secondary outcomes were collected after 6,12 and 24 months Mixed-model multi-level analyses showed no significant differences between the two groups with regard to all primary (disability) and secondary outcomes (i.e., depressive symptomatology, social support interactions, fear of falling, social participation and quality of life). Pre-planned subgroup analyses confirmed these results. Consequently, no evidence for the effectiveness of the PoC approach was found.

Third, an economic evaluation was conducted to evaluate the cost-effectiveness of the PoC approach (Chapter 7). We hypothesised that the programme should lead to reduced disability, improved quality of life and reduced healthcare use and related costs. A cost-effectiveness (CEA) and cost-utility analysis (CUA) were performed from a societal perspective with a time horizon of 24 months. Healthcare utilisation was measured continuously during 24 months. Daily functioning and quality of life were measured at baseline and after 6,12 and 24 months follow-up. Bootstrap analyses were performed to estimate uncertainty of the findings, and sensitivity analyses were conducted to assess the generalisability of assumptions made. People in the intervention group had significantly more contacts with the practice nurse $(5.2$ versus $1.1, p<0.001)$ and had more occupational therapy sessions $(7.1$ versus $1.2, p<0.001$ ) than older people in the control group. Remaining categories of healthcare utilisation were similar between the groups. Complete cases (56\%) were included in the CEA and CUA analyses. Total healthcare costs in the intervention group were 26,503 compared to 20,550 in the control group. Despite these highe costs in the intervention group we found no significant differences between the groups with regard to disability and quality of life.

Chapter 8 provides a summary and discussion of the main findings, followed by implications for practice and future research.

There is no evidence for the effectiveness and cost-effectiveness of the PoC approach. Possibly the wrong target group was recruited for this study. There is still a ot of discussion in the literature about the concept of frailty and frailty instruments with sufficient psychometric properties are lacking. Another reason might be the 
inadequate implementation of the intervention protocol during the study period. Although participating older people and professionals were satisfied with the approach, some parts were not implemented as planned, as professionals experienced difficulties in applying them into daily practice. Multiple and complex healthcare needs of frail older people have to be addressed by complex interventions. However, we have to critically consider how much complexity healthcare professionals can handle and how to design interventions that are as simple and straightforward as possible. Intense training programme are needed to change well-established patterns of care. Consequently, successful interventions have to focus on both patients and professionals, while taking into account their environmental circumstances. Due to lack of effects and higher costs compared to usual care, the implementation of the PoC approach in its current form is not recommended, at least not in the Netherlands or in other countries with similar healthcare systems. Future research should focus on the conceptualisation and measurement of frailty and (cost-) effective interventions for the target group of community-dwelling frail older people.

In conclusion, the aim of this thesis was to provide evidence for the feasibility effectiveness and cost-effectiveness of the PoC approach. Although, no positive results were found this thesis provided valuable knowledge with regard to the complexity of interdisciplinary primary care for frail older people and describes methodologica challenges in this field of research. 


\section{Samenvatting}

Evaluatie van Zorg uit Voorzorg

Dit proefschrift beschrijt de evaluatie van een interdisciplinair eerstelijnsprogramma voor thuiswonende ouderen in een kwetsbare positie: "Zorg uit Voorzorg". Het doel van dit programma is het reduceren van beperkingen in activiteiten van het dagelijkse even en het voorkomen van (verdere) functionele achteruitgang. Zorg uit Voorzorg bestaat uit een zestal stappen: (1) eerst vindt een schriftelijke screening met behulp van de "Groningen Frailty Indicator" plaats om ouderen in een kwetsbare positie op e sporen; (2) vervolgens krijgen de opgespoorde ouderen tijdens een huisbezoek een uitgebreid onderzoek door een praktijkondersteuner en eventueel aanvullende onderzoek door andere disciplines zoals ergotherapeut or geriater; (3) daarna stellen praktijkondersteuner en huisarts samen een voorlopig plan van aanpak op. Bij complexe problematiek wordt het team uitgebreid met andere disciplines (bijvoorbeeld fysiotherapeut of ergotherapeut); in dat geval vindt multidisciplinair overleg plaats (4) de praktijkondersteuner stelt samen met de oudere tijdens een tweede huisbezoek een definitief plan van aanpak vast. Dit plan bevat doelen, strategieën en acties die gerelateerd zijn aan een toolbox van interventies. De toolbox bestaat uit een combinatie van medische en meer sociaal (participatie/activiteiten) gerichte interventies. (5) De praktijkondersteuner, huisarts, fysiotherapeut en ergotherapeut zijn bij de uitvoering van de toolbox onderdelen betrokken. Daarnaast kan een beroep gedaan worden op andere hulpverleners en voorzieningen in de eerste of tweede lijn; (6) het bereiken van doelen en de implementatie van strategieën in het dagelijkse leven worden regelmatig geëvalueerd en er worden afspraken gemaakt voor follow-up (zie Appendix voor een gedetailleerde beschrijving van het programma).

Hoofdstuk 1 gaat in op de concepten "kwetsbaarheid" en "beperkingen in activiteiten van het dagelijkse leven" bij ouderen. Bovendien wordt het belang van eerstelijnszorg voor ouderen in een kwetsbare positie aangegeven en recente ontwikkelingen in de Nederlandse gezondheidszorg beschreven. Het hoofdstuk eindigt met de hoofddoelstellingen van dit proefschrift.

Om beperkingen in activiteiten van het dagelijkse leven te verminderen en (verdere) functionele achteruitgang te voorkomen, is het van belang om ouderen in een kwetsbare positie tijdig op te sporen. Hiervoor zijn valide screeningsinstrumenten nodig. Tot op heden is er echter weinig wetenschappelijke ondersteuning voor de psychometrische kwaliteit van bestaande instrumenten. Een valideringstudie (Hoofdstuk 2) is uitgevoerd om de kwaliteit van drie vragenlijsten te evalueren en te vergelijken: de "Groningen Frailty Indicator" (GFI), de "Tilburg Frailty Indicator" (TFI) en de "Sherbrook Postal 
Questionnaire" (SPQ). De GFI en de TFI hebben in vergelijking met de SPQ een hoge interne consistentie en construct validiteit laten zien. De resultaten laten het echter niet toe om eenduidige conclusies te trekken of de GFI of de TFI de voorkeur verdient. Wel kan worden geconcludeerd dat de SPQ minder geschikt is.

In de laatste decennia is er veel onderzoek gedaan naar interventies voor thuiswonende ouderen in een kwetsbare positie. Er zijn diverse meta-analyses en systematische reviews uitgevoerd die zich richten op specifieke categorieën van interventies; het betreft vooral preventieve huisbezoeken en fysieke oefenprogramma's. Echter, er bleek geen studie te zijn die een volledig beeld geeft van bestaande interventies me "beperkingen in activiteiten van het dagelijkse leven" als uitkomstmaat. Daarom is een narratieve review (Hoofdstuk 3) uitgevoerd om veelbelovende componenten voor toekomstige interventies te achterhalen, gericht op het verminderen van beperkingen in dagelijkse activiteiten en het voorkomen van (verdere) achteruitgang in functioneren bij ouderen. Gebaseerd op deze review wordt aanbevolen dat toekomstige interventies voor thuiswonende ouderen in een kwetsbare positie uitgevoerd moeten worden door een interdisciplinair eerstelijnsteam. Het programma zou de volgende elementen moeten bevatten: een screening, individueel onderzoek met bijbehorende specifieke interventies (zorg op maat), zelfmanagement ondersteuning, het identificeren en werken aan betekenisvolle activiteiten, casemanagement en lange termijn follow-up. Deze veelbelovende elementen zijn gecombineerd in het programma Zorg uit Voorzorg.

Om de praktische uitvoerbaarheid, effectiviteit en kosteneffectiviteit van Zorg uit Voorzorg te evalueren is er een cluster gerandomiseerd onderzoek uitgevoerd in twaalf huisartspraktijken in de Westelijke Mijnstreek. Deze praktijken zijn at random toegewezen aan de interventiegroep (zes praktijken, Zorg uit Voorzorg) of controlegroep (zes praktijken, reguliere zorg) (Hoofdstuk 4). Een schriftelijke screeningslijst bestaande uit de GFI, is naar 3.498 ouderen ( $\geq 70$ jaar) gestuurd. Het responspercentage was $80 \%$. Van de ouderen die bereid waren om deel te nemen aan he onderzoek, bevonden zich volgens de GFI (GFI $\geq 5) 36 \%$ in een kwetsbare positie. Uiteindelijk zijn er 346 ouderen geïncludeerd in het onderzoek dat uit drie samenhangende deelstudies bestaat: een procesevaluatie, een effectevaluatie en een economische evaluatie.

Ten eerste is er een uitgebreide procesevaluatie uitgevoerd (Hoofdstuk 5). Een mixed methods design is toegepast om inzicht te verkrijgen in hoeverre Zorg uit Voorzorg volgens protocol is uitgevoerd. Bovendien zijn de ervaringen van hulpverleners en ouderen met het programma geëvalueerd en zijn belemmerende en bevorderende factoren geïnventariseerd. Gegevens voor de procesevaluatie zijn verzameld bi ouderen in de interventiegroep en betrokken hulpverleners. Hulpverleners waren ove het algemeen tevreden over Zorg uit Voorzorg, omdat het een goede structuur geeft aan de invulling van de geriatrische zorg in de eerste lijn en de aandacht verhoog voor interdisciplinair samenwerken en preventieve zorg. Ouderen in een kwetsbare positie voelden zich begrepen door de hulpverleners en hebben steun ervaren bij het omgaan met hun problemen en het bereiken van betekenisvolle doelen die binnen het programma werden geformuleerd. Door de complexiteit van het interventieprotoco zijn sommige onderdelen echter niet goed gevolgd: (1) de voorlopig plan van aanpak is vaak niet tijdens een bilateraal overleg of een teambiijeenkomst besproken (stap 3); (2) slechts de helft van de deelnemende ouderen heeft een tweede huisbezoek ontvangen om het plan van aanpak te bespreken (stap 4); (3) de zogenaamde ( beperkt aantal evaluatie- en follow-up momenten (stap 6).

Ten tweede zijn de effecten van het programma Zorg uit Voorzorg op beperkingen in activiteiten van het dagelijkse leven (primaire uitkomstmaat) en diverse secundaire uitkomstmaten onderzocht (Hoofdstuk 6). In de interventiegroep (zes praktijken) hebben 193 ouderen het programma Zorg uit Voorzorg ontvangen. Deze zijn vergeleken met 153 ouderen in de controlegroep die reguliere zorg ontvingen. De gemiddelde leeftijd van de gehele onderzoeksgroep was 77,2 jaar; $58 \%$ was vrouw $49 \%$ was alleenstaand en $58 \%$ had een lage opleiding. Bij de start van het onderzoek waren ouderen in de interventiegroep gemiddeld meer kwetsbaar en beperkt dan ouderen in de controlegroep. Na 6 maanden nam 91\% van de ouderen nog deel aan het onderzoek, na 12 maanden was het percentage 86\% en na 24 maanden $78 \%$, met significant meer uitval in de interventiegroep. Gegevens betreffende de primaire en secundaire uitkomstmaten zijn verzameld na 6, 12 en 24 maanden. Uit de resultaten blijkt dat er geen significante verschillen zijn tussen de groepen met betrekking tot alle primaire (beperkingen in activiteiten van het dagelijkse activiteiten) en secundaire uitkomstmaten (depressieve symptomen, sociale steun interacties, bezorgdheid om te vallen, sociale participatie en kwaliteit van leven). De voora geplande subgroep analyses laten geen andere bevindingen zien. Er kan worden geconcludeerd dat er voor deze uitkomsten geen empirisch bewijs is voor de effectiviteit van Zorg uit Voorzorg.

Ten derde is een economische evaluatie uitgevoerd om de kosteneffectiviteit van Zorg uit Voorzorg te evalueren (Hoofdstuk 7). De aanname vooraf was dat het programma zou leiden tot minder beperkingen in activiteiten van het dagelijkse leven, een hogere kwaliteit van leven en over het geheel genomen minder gebruik van zorg en gerelateerde kosten. Vanuit een maatschappelijk perspectief met een tijdshorizon van 24 maanden zijn er kosten-effectiviteitsanalyses (KEA) en kosten-utiliteitsanalyses (KUA) uitgevoerd. Zorggebruik is gedurende een periode van 24 maanden 
geregistreerd. Beperkingen in activiteiten van het dagelijkse leven en kwaliteit van leven zijn gemeten aan het begin van de studie en na 6, 12 en 24 maanden. Bootstrap analyses zijn uitgevoerd om de onzekerheid rondom de resultaten te schatten. Bovendien zijn er sensitiviteitsanalyses gedaan om de generaliseerbaarheid van de gemaakte aannames te onderzoeken. Ouderen in de interventiegroep hadden significant meer contacten met de praktijkondersteuner (5,2 contacten versus 1,1 contacten, $\mathrm{p}<0,001)$ en gebruikten ook significant meer ergotherapie $(7,1$ sessies versus 1,2 sessies, $p<0,001)$ dan ouderen in de controlegroep. Het gebruik van andere typen zorg was vergelijkbaar tussen de twee groepen. Alleen de ouderen voor wie alle gegevens gedurende de 24 maanden beschikbaar waren (56\%) zijn geïncludeerd in de KEA en KUA analyses. De totale kosten voor het gebruik van zorg bedroegen per persoon 26.503 in de interventiegroep en 20.550 in the controlegroep. Er zijn geen significant verschillen tussen de groepen gevonden met betrekking to beperkingen in activiteiten van het dagelijkse leven en kwaliteit van leven.

Hoofdstuk 8 bevat de conclusies van het onderzoek, evenals een discussie van de bevindingen. Tevens worden aanbevelingen voor de praktijk en toekomstig onderzoek geformuleerd.

Er is geen empirisch bewijs voor de effectiviteit en kosteneffectiviteit van Zorg uit Voorzorg. Een verklaring zou kunnen zijn dat niet de goede doelgroep voor he onderzoek gerekruteerd is. Er is nog steeds veel onduidelijkheid rondom het begrip kwetsbaarheid en meetinstrumenten op dit terrein met goede psychometrische eigenschappen zijn nog onvoldoende voorhanden. Maar het ontbreken van effecten zou ook te maken kunnen hebben met onvoldoende implementatie van Zorg uit Voorzorg tijdens de onderzoeksperiode. Ofschoon de deelnemende ouderen en hulpverleners over het algemeen tevreden waren over het programma, zijn sommige onderdelen van het protocol niet goed uitgevoerd, omdat ze als moeilijk toepasbaar ervaren zijn. De meervoudige en complexe zorgbehoeftes van ouderen in een kwetsbare positie vragen om complexe interventies. Echter, een punt van aandacht blijft de vraag hoeveel complexiteit hulpverleners aan kunnen en hoe interventies op een zo eenvoudig mogelijke wijze kunnen worden aangeboden. Er is een behoefte aan intensieve scholingsprogramma's om gevestigde patronen van zorgverlening te veranderen. Als gevolg zouden succesvolle interventies zich moeten richten op zowe ouderen als hulpverleners rekening houdend met de fysieke en sociale omgeving waar ouderen in verblijven en hulpverleners in werken. Gezien er geen effecten op de primaire en secundaire uitkomstmaten gevonden zijn en Zorg uit Voorzorg geleid heeft tot meer (zorg-) kosten, wordt aanbevolen om het programma in de huidige vorm niet te implementeren. Dit geldt in ieder geval voor Nederland of andere landen met een vergelijkbaar gezondheidszorgsysteem. Toekomstig onderzoek zou zich eerst moeten richten op het conceptualiseren en het meten van kwetsbaarheid en (kosten-) effectieve interventies voor de doelgroep of thuiswonende kwetsbare ouderen.

Concluderend: het doel van dit proefschrift was het genereren van empirisch bewijs voor de praktische toepasbaarheid, effectiviteit en kosteneffectiviteit van he programma Zorg uit Voorzorg. Ofschoon er met betrekking tot (kosten-) effectivitei geen positief bewijs is gevonden, heeft het onderzoek waardevolle inzichten opgeleverd waar het gaat om de complexiteit van interdisciplinaire eerstelijnszorg voor ouderen in een kwetsbare positie. Tevens zijn verschillende methodologische uitdagingen geformuleerd. 


\section{Zusammenfassung}

Evaluation des „Prevention of Care" Ansatzes

Die vorliegende Dissertation beschreibt eine cluster-randomisierte Interventionsstudie in der ein interdisziplinärer ambulanter Versorgungansatz für gebrechliche ältere Menschen evaluiert wird: der „Prevention of Care“ (PoC) Ansatz. Zielsetzung dieses Versorgungsansatzes ist zum einen, bestehende funktionelle Einschränkungen, die mit der Gebrechlichkeit einhergehen, zu reduzieren und zum anderen, (weiteren) Einschränkungen vorzubeugen, sodass ältere Menschen trotz ihrer Gebrechlichkeit die Anforderungen des alltäglichen Lebens meistern und länger in ihrem gewohnten Lebensumfeld verbleiben können.

Der PoC-Ansatz besteht aus sechs Schritten: (1) im ersten Schritt findet ein GebrechlichkeitsScreening statt. Ältere Menschen ( $\geq 70$ Jahre) erhalten per Post einen schriftlichen Fragebogen, den „Groningen Frailty Indicator”; (2) Personen, die als gebrechlich identifiziert wurden, werden während eines Hausbesuches einer ausführlichen Diagnostik unterzogen, die durch die medizinische Fachangestellte des Hausarztes („practice nurse“) ausgeführt wird. Bei Bedarf können diagnostische Verfahren durch andere medizinische Fachkräfte erfolgen (z.B. Ergotherapeuten oder Geriater); (3) danach erstellen der Hausarzt und die medizinische Fachangestellte gemeinsam einen vorläufigen Behandlungsplan. Bei komplexen Problemen werden andere medizinische Fachkräfte hinzugezogen (z.B. Physiotherapeuten, Ergotherapeuten oder Apotheker). In diesem Fall findet eine interdisziplinäre Teambesprechung statt; (4) im vierten Schritt besucht die medizinische Fachangestellte den Patienten ein zweites Mal, um einen definitiven Behandlungsplan zu erstellen. Der Behandlungsplan beinhaltet Ziele, Strategien und Handlungsempfehlungen. Für die Behandlung steht eine Toolbox mit Interventionen zur Verfügung. (5) Die Toolbox-Interventionen werden im nächsten Schritt je nach Bedarf durch die Medizinische Fachangestellte den Physiotherapeuten, den Ergotherapeuten oder den Hausarzt ausgeführt. Zudem können andere medizinische Fachangestellte und Institutionen konsultiert werden (6) abschließend wird das Erreichen der Behandlungsziele und die Umsetzung der Strategien ins alltägliche Leben evaluiert. Außerdem werden Absprachen hinsichtlich der Nachsorge getroffen (siehe Appendix für eine detaillierte Beschreibung des PoCAnsatzes).

In Kapitel 1 werden die Konzepte der "Gebrechlichkeit" und der „funktionellen Beeinträchtigungen" erläutert. Des Weiteren wird die Bedeutung der ambulanten Versorgung gebrechlicher älterer Menschen thematisiert, die es innen ermöglich soll, trotz ihrer Gebrechlichkeit die Anforderungen des alltäglichen Lebens zu meistern 
und in ihrem gewohnten Lebensumfeld zu verbleiben. Zudem werden gegenwärtige Entwicklungen im Niederländischen Gesundheitswesen beschrieben. Das Kapitel endet mit einer Beschreibung der Hauptzielsetzungen dieser Dissertation.

Um bestehende funktionelle Einschränkungen, die mit der Gebrechlichkeit einhergehen, zu reduzieren und (weiteren) Einschränkungen vorzubeugen, müssen gebrechliche ältere Menschen frühzeitig identifiziert werden. Hierfür werden valide Screenings Instrumente benötigt. Es gibt jedoch kaum empirische Grundlage hinsichtlich der psychometrischen Eigenschaften bestehender Instrumente. In einer Validationsstudie (Kapitel 2) wurden die Eigenschaften dreier Fragebögen evaluiert und miteinande verglichen: der "Groningen Frailty Indicator" (GFI), der „Tilburg Frailty Indicator" (TFI) und der "Sherbrook Postal Questionnaire" (SPQ). Dem GFI- und TFI-Fragebogen sind im Vergleich zum SPQ-Fragebogen eine hohe interne Konsistenz- und Konstruktvalidität zu zuschreiben. Es bleibt jedoch unklar, ob GFI oder TFI bevorzugt werden sollten. Lediglich der SPQ-Fragebogen scheint weniger angemessen zu sein.

In den letzten Jahrzehnten wurden zahlreiche Interventionsstudien bei gebrechlichen älteren Menschen durchgeführt. Obschon einige Meta-Analysen und systematische Reviews erschienen sind, richten sich diese zumeist auf spezifische Interventionskategorien, meistens im Bereich von physischen Trainingsprogrammen oder präventiven Hausbesuchen. Es gab keine Literaturstudie, welche die gesamte Bandbreite an ambulanten Interventionen zur Behandlung von funktionellen Einschränkungen wiederspiegelt. Demzufolge wurde ein narrativer Review ausgeführt (Kapitel 3) um vielversprechende Komponenten für zukünftige Interventionen herauszufiltern. Basierend auf diesem Review sollte ein ambulanter Versorgungsansatz für gebrechliche ältere Menschen der durch ein interdisziplinäres Team angeboten wird, entwickelt werden. Des Weiteren scheinen ein Gebrechlichkeits-Screening, individuelle Diagnostik und Interventionen, Unterstützung bei Selbstmanagement, Betätigung in bedeutungsvollen Aktivitäten, Case Management und Langzeitnachsorge vielversprechende Komponenten zu sein. Diese Komponenten wurden im PoC Ansatz vereinigt.

Um die praktische Ausführbarkeit, Effektivität und Kosteneffektivität des PoC Ansatzes zu evaluieren wurde eine cluster-randomisierte Interventionsstudie in zwölf Hausarztpraxen in Sittard (NL) und Umgebung ausgeführt. Die Praxen wurden per Zufall der Interventionsgruppe (sechs Praxen, PoC Ansatz) oder Kontrollgruppe (sechs Praxen, reguläre Versorgung) (Kapitel 4) zugeteilt. Ein schriftlicher Fragenbogen (GFI) wurden an 3.498 ältere Menschen ( $\geq 70$ Jahre) verschickt. Die Antwortrate des schriftlichen Screenings war $80 \%$. Von den Personen, die bereit waren an der Studie teilzunehmen, waren laut des GFI-Fragebogens (GFI $\geq 5$ ) 36\% gebrechlich. Letztendlich haben 346 gebrechliche ältere Menschen an der Studie teilgenommen, die aus drei Teilstudien besteht: einer Prozessevaluation, einer Effektivitätsstudie und einer ökonomischen Evaluation.

Als erstes, wurde mit Hilfe eines Mixed Methods Design eine umfassende Prozessevaluation ausgeführt (Chapter 5) um zu beurteilen, ob bei der Anwendung des PoC-Ansatz gemäß Protokoll ausgeführt wurde. Des Weiteren wurden die Erfahrungen der gebrechlichen älteren Menschen und des interdisziplinären Teams (Hausärzte, medizinischen Fachangestellten, Physiotherapeuten und Ergotherapeuten) im Hinblick auf unterstützende und hinderliche Faktoren evaluiert. Das interdisziplinäre Team war im Allgemeinen zufrieden mit dem PoC-Ansatz, da er eine gute Struktur für die ambulante geriatrische Versorgung bot und zudem die Aufmerksamkeit bezüglich interdisziplinärer Zusammenarbeit und Prävention erhöhte. Teilnehmende gebrechliche ältere Menschen fühlten sich verstanden und haben Unterstützung bei dem Erreichen ihrer Ziele erfahren, die im Rahmen des PoC-Ansatzes erstellt wurden. Durch die Komplexität des Interventionsprotokolls wurden manche Aspekte der Intervention nicht wie gewünscht ausgeführt: (1) der vorläufige Behandlungsplan wurde oftmals nicht während eines Teammeetings besprochen (Schritt 3); (2) nur die Hälfte der gebrechlichen älteren Menschen haben einen zweiten Hausbesuch erhalten um den definitiven Behandlungsplan zu besprechen (Schritt 4); (3) die sogenannte Toolbox wurde nicht oft angewendet (Schritt 5); und (4) es gab nur wenige Evaluations- und Nachsorgemomente (Schritt 6)

Als Zweites, wurden die Effekte des PoC Ansatzes im Hinblick auf funktionelle Beeinträchtigungen (primärer Endpunkt) und diverse sekundäre Endpunkte untersucht (depressive Symptome, soziale Unterstützung, Angst vor dem Fallen, soziale Partizipation und Lebensqualität) (Kapite/ 6). In der Interventionsgruppe (sechs Praxen) wurden 193 gebrechliche ältere Menschen nach dem PoC-Ansatz behandelt. Diese wurden verglichen mit 153 gebrechlichen älteren Menschen in de Kontrollgruppe, die die reguläre Versorgung erhielten. Das durchschnittliche Alter der gesamten Stichprobe war 77,2 Jahre, 58\% waren weiblich, 49\% waren alleinstehend und 58\% hatten eine niedrige Schulbildung. Zu Beginn der Studie waren die gebrechlichen älteren Menschen in der Interventionsgruppe im Durchschnitt gebrechlicher und funktionell mehr beeinträchtigt als die Personen in der Kontrollgruppe. Nach 6 Monaten nahmen noch 91\% der gebrechlichen älteren Menschen an der Studie teil nach 12 Monate war der Prozentsatz auf 86\% und nach 24 Monaten auf 78\% gesunken, wobei in der Interventionsgruppe signifikant mehr Menschen die Studie frühzeitig beendet haben als in der Kontrollgruppe. Daten im Hinblick auf die primären und sekundären Endpunkte sind zu Beginn und nach 6, 12 und 24 Monaten erhoben worden. Basierend auf den Ergebnissen dieser Studie gab es keine signifikanten 
Unterschiede zwischen den Gruppen bezüglich aller primären und sekundären Endpunkte. Die vorab geplanten Subgruppenanalysen verifizieren diese Ergebnisse. Auf Grund dessen ist die Schlussfolgerung, dass es keinen empirischen Beweis für die Effektivität des PoC-Ansatzes gibt

Als drittes wurde eine ökonomische Evaluation durchgeführt um die Kosteneffektivität des PoC-Ansatzes zu beurteilen (Kapitel 7). Es wurde angenommen, dass der PoC-Ansatz funktionelle Beeinträchtigung reduziert und die Lebensqualität erhöht. Zudem sollte er im Allgemeinen die Inanspruchnahme von Versorgungsleistungen reduzieren und folglich geringere Kosten verursachen. Es wurde eine KostenWirksamkeits-Analyse und Kosten-Nutzwert-Analyse ausgeführt, für diese Analyse wurde eine gesellschaftliche Perspektive und ein Zeitrahmen von 24 Monaten gewählt. Hierfür wurde in beiden Gruppen die Inanspruchnahme der Versorgungsleistungen in der 24-monatigen Studienperiode registriert. Daten bezüglich der funktionellen Beeinträchtigungen und Lebensqualität wurden zu Beginn der Studie, nach 6, 12 und 24 Monaten erhoben. Zur Validierung der Ergebnisse wurde eine Bootstrap Analyse angewendet. Mit Hilfe von Sensitivitätsanalysen wurde die Generalisierbarkeit der gemachten Annahmen evaluiert. Die ökonomische Evaluation zeigte, dass gebrechliche ältere Menschen in der Interventionsgruppe signifikan mehr Kontakte mit medizinischen Fachangestellten (5,2 Kontakte versus 1,1 Kontakte $p<0,001)$ und Ergotherapeuten (7,1 Einheiten versus 1,2 Einheiten, $p<0,001)$ hatten als die Personen aus der Kontrollgruppe. Die Inanspruchnahme andere Versorgungsformen war vergleichbar zwischen den Gruppen. Die gebrechlichen älteren Menschen, bei denen ein vollständiger Datensatz vorlag (56\%), wurden in die Kosten-Wirksamkeits- und Kosten-Nutzwert-Analysen inkludiert. Die totalen Versorgungskosten in 24 Monaten waren pro Kopf 26.503 in der Interventionsgruppe und 20.550 in der Kontrollgruppe. Es gab keine signifikanten Unterschiede hinsichtlich funktioneller Beeinträchtigung und Lebensqualität.

Kapitel 8 beinhaltet die Schlussfolgerungen dieser Studie und eine Diskussion de Ergebnisse. Außerdem werden Handlungsempfehlungen für die Praxis und zukünftige Studien ausgesprochen.

Es gibt keine empirischen Beweise für die Effektivität und Kosteneffektivität des PoC-Ansatzes. Möglicherweise wurde in der vorliegenden Studie nicht die richtige Zielgruppe für den gewählten Ansatz rekrutiert. Dies kann unter anderem damit zusammenhängen, dass in der Fachliteratur noch nicht klar definiert ist was unter dem Begriff der Gebrechlichkeit verstanden wird. Des Weiteren sind GebrechlichkeitsInstrumente mit guten psychometrischen Eigenschaften nur unzureichend vorhanden. Jedoch kann das Fehlen von Effekten auch mit der mangelhaften Umsetzung des PoC-Ansatzes während der Studienperiode zusammenhängen. Obwohl die teil-nehmenden gebrechlichen älteren Menschen und das interdisziplinäre Team im Allgemeinen mit dem PoC-Ansatz zufrieden waren, wurden einige Aspekte des Protokolls nicht wie gewünscht ausgeführt, da sie in ihrer praktischen Handhabung als schwierig empfunden wurden. Die Anzahl und Komplexität der Versorgungsbedarfe gebrechlicher älterer Menschen erfordert komplexe Interventionen. Jedoch bleibt es fraglich, wie viel Komplexität durch medizinische Fachkräfte umgesetzt werden kann und wie Interventionen so einfach wie möglich gestaltet werden können. Es gibt einen Bedarf an intensiven Fortbildungsprogrammen um gefestigte Routinen in der Versorgung zu verändern.

Da hinsichtlich der primären und sekundären Endpunkte keine Effekte gefunden werden konnten wird empfohlen, den PoC-Ansatz in der heutigen Form nicht in der Praxis einzuführen. Diese Empfehlung gilt zumindest für die Niederlande und andere Länder mit vergleichbaren Gesundheitssystemen. Zukünftige Studien sollten sich mit dem Konzept der Gebrechlichkeit und der Validierung von GebrechlichkeitsInstrumenten beschäftigen. Zudem werden (kosten-) effektive ambulante Versorgungsansätze für gebrechliche ältere Menschen benötigt, um ihnen zu

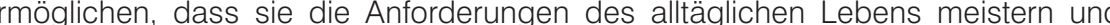
länger in ihrem gewohnten Lebensumfeld verbleiben können

Zusammenfassend: Das Ziel dieser Dissertation war es, empirische Beweise für die Ausführbarkeit, Effektivität und Kosteneffektivität des PoC-Ansatzes zu erbringen Obschon hinsichtlich der (Kosten-) Effektivität keine positiven Resultate gefunden wurden, ergeben sich aus dieser Studie wertvolle Einblicke in die Komplexität der interdisziplinären ambulanten Versorgung gebrechlicher älterer Menschen. Des Weiteren werden verschiedene methodologische Herausforderungen beschrieben. 


\section{Dankwoord | Dankwort}

De laatste loodjes wegen het zwaarst ... volgens mij geldt dit in het bijzonder voor he schrijven van een dankwoord. De afgelopen jaren ben ik veel mensen tegen gekomen die interesse in mijn onderzoek getoond hebben, me gemotiveerd en gesteund hebben en die tijd en energie in dit onderzoek gestoken hebben. ledereen heeft op zijn of haar eigen manier, een steentje bijgedragen aan de totstandkoming van dit proefschrift. Mijn dank hiervoor!

Graag wil ik een aantal mensen persoonlijk bedanken.

Allereerst de deelnemers aan dit onderzoek: ouderen, mantelzorgers, huisartsen praktijkondersteuners, ergotherapeuten en fysiotherapeuten. Zonder jullie bereidheid om mee te werken aan dit onderzoek was dit proefschrift er nooit gekomen.

Vervolgens zou ik graag mijn promotoren Ruud Kempen en Luc de Witte, en co-promotor Erik van Rossum willen danken. Voor mij waren jullie het perfecte promotieteam. ledereen heeft vanuit zijn expertise input geleverd aan dit proefschrift. Hierbij hebben jullie elkaar uitstekend aangevuld. Vanaf het begin waren jullie een bron van inspiratie voor mij en gedurende het heel traject heb ik veel van jullie mogen leren. Ook hebben we vaak gelachen tijdens onze driewekelijkse overleggen, wat heb ik hiervan genoten! Beste Ruud, het is ongeveer 6 jaar geleden dat ik als fris afgestudeerde HBO student voor het eerst bij je binnenliep, maar met je hartelijke karakter en je aanmoedigende woorden wist je snel mijn onzekerheid weg te nemen. Je wetenschappelijke deskundigheid, je persoonlijke gedrevenheid, en je oog voo detail zijn eigenschappen die ik heel erg waardeer. Bovendien staat je deur altijd open wat niet vanzelfsprekend is bij zulke drukbezette mensen. Dankjewel voor je grote inzet, je vertrouwen in mij en dat je me de mogelijkheden biedt om me steeds verder te ontwikkelen. Beste Luc, als tweede promotor heb je de grote lijnen bewaak en met je frisse en kritische blik wist je vaak een draai aan onze discussies te geven en de puntjes op de "I" te zetten. Mijn dank hiervoor. Beste Erik, vanaf het eerste uu heb je me - toen nog masterstudente en later promovendi - ontzettend goed begeleid. Jouw feedback was altijd helder en constructief en je wiste het zo te brengen dat ik nooit met een slecht gevoel naar buiten ging. Ik waardeer je pragmatische aanpak die gepaard gaat, met belangstelling voor wetenschap en praktijk en een grote portie humor. Erik en Ruud, ik ben heel erg blij dat we ook in komende jaren ons werk kunnen voortzetten. Luc, we zullen ons beslist blijven zien en wie weet misschien staat ooit weer een gemeenschappelijk project op ons te wachten. 
Mijn dank gaat ook uit naar de leden van mijn projectgroep/ co-auteurs, die een belangrijke bijdrage hebben geleverd aan dit proefschrift: Sjoerd Hobma, Walther Sipers, Herbert Habets, Lilo Crasborn, Simone Denis, Marlou Wolters, Ton Ambergen, Karen Cox, Marike Hendriks en Ramon Daniëls. Beste Ramon, na de opleiding ergotherapie heb je me de weg naar de Universiteit Maastricht gewezen. Vervolgens gaf je me tijdens mijn masteropleiding de kans om als studentassistente een kijkje in de 'onderzoekskeuken' te nemen. Je was niet alleen nauw betrokken bij mijn masteronderzoek, maar ook later heb je als lid van de projectgroep altijd enthousiasme en betrokkenheid getoond. Beste Marike, jouw inzet bij de economische evaluatie was buitengewoon. Het artikel over zorggebruik en kosten was een beetje mijn zorgen-kindje, maar dankzij jouw voortdurende inzet en motivatie is er uiteindelijk een mooi artikel tot stand gekomen. Je stond me altijd met raad en daad terzijde en dat waardeer ik zeer! Beste Sjoerd, Walther, Herbert, Lilo, Simone en Marlou, geen veldonderzoek zonder mensen die dicht bij de praktijk staan. Jullie hebben ontzettend veel inspanningen verricht om dit project van de grond te krijgen en de boel draaiende te houden. Ton, bedankt voor hulp en adviezen bij de statistische analyses. Karen mijn dank voor je steun bij de kwalitatieve data verzameling en analyse.

Beste collega's van de vakgroep Health Services Research, bedankt voor jullie belangstelling in mijn proefschrift, jullie constructieve feedback op mijn werk tijdens diverse refereerbijeenkomsten, de leuke gesprekken op de wandelgangen en de gezellige momenten tijdens uitstapjes en congresbezoeken. Beste Bart, beste Jill, sinds 2009 deelden we niet alleen een werkkamer, maar ook lief en leed. Jullie hadden altijd een luisterend oor en stonden me met goede adviezen ter zijde. Me een lach en een traan kijk ik uit naar onze promoties. Ik ben blij dat we het voor elkaa gekregen hebben om allemaal binnenkort te promoveren, maar ik ga jullie ook ontzettend missen op Dub30! Een belangrijke bron van steun was ook ons team van onderzoeksassistenten: Ine Hesdahl, Astrid Dello en Floor Komen. Samen met Bar en Jill vormden we het 'Tp1 team'. De dataverzameling stelde ons voor veel logistieke uitdagingen, maar met zijn vijven wisten we alles tot een goed einde te brengen. Bovendien was het in ons team ontzettend gezellig - ook buiten het werk. Ik kijk terug op diverse leuke avonden in Maastricht, Aken of Mönchengladbach. Beste Bart, Jil, Ine, Astrid en Floor, bedankt voor deze mooie momenten en de prettige samenwerking in de afgelopen jaren.

Bij de dataverzameling waren ook enige 'externe partners' betrokken, die belangrijk werk hebben verricht. Wilma Krämer, bedankt dat je in de registratie van Orbis Medisch Centrum bent gedoken om voor de deelnemers van onze studie de gegevens met betrekking tot ziekenhuisopnames en poliklinische bezoeken op te zoeken. Dank gaat ook uit naar Rob Doktor en Daniëlle Baenen van CZ, Herm Jan Mateboer en
Gerard Timmermans van Achmea en Marc Korzilius van VGZ. Jullie hebben de gegevens met betrekking tot het eerstelijns zorggebruik voor jullie rekening genomen. Beste medewerkers van MEMIC, in het bijzonder Marlène Ronner, Alfons Schroten en Anita Legtenberg, bedankt voor jullie hulp bij de logistiek rondom de dataverzameling en het afnemen respectievelijk invoeren van talrijke telefonische interviews en vragenlijsten

Ook wil ik de leden van de beoordelingscommissie van dit proefschrift hartelijk danken voor het lezen en beoordelen van dit proefschrift: prof. dr. T. van der Weijden, prof. dr. med. A. Stuck, prof. dr. M. Schuurmans, prof. dr. C. Dirksen, onder voorzitterschap van prof. dr. J. Metsemakers.

Mijn dank gaat ook uit naar ZonMw, NWO, the School for Public Health and Primary Care (CAPHRI) en Zuyd Hogeschool voor het financieren van deze studie, diverse open-acces publicaties en een verblijf in het buitenland gedurende mijn promotietraject.

Liebe Nicole, Eva, Rosalie, Sabrina D., Frauke, Jessica, Sabrina Z., Steffi, Moni und Ania, vielen Dank für Eure langjährige Freundschaft. Die meisten von Euch kenne ich schon fast mein ganzes Leben und ich weiß es unheimlich zu schätzen, dass unsere Freundschaft über all die Jahre hinweg Bestand hat. Ich möchte Euch von Herzen danken für das Interesse in meine Arbeit, die aufmunternden Worte und Eure langjährige Freundschaft. Ich kann es kaum erwarten mit Euch anzustoßen und diesen Meilenstein in meinem Leben zu feiern!

Liebe Mama, lieber Papa, mit Stolz schaue ich auf Euer Leben. Seit nunmehr 40 Jahre geht ihr durch dick und dünn und meistert alle Lebenslagen. Euer Lebenszie war es immer, Christina und mir einen guten Start in ein eigenständiges Leben zu ermöglichen. Hierfür habt Ihr hart gearbeitet und auf vieles verzichtet und dies sollte sich letztendlich auszahlen. Sowohl Christina als auch ich haben unseren Weg gefunden und unser Erfolg ist Euer Erfolg, denn ohne Euch wäre das niemals möglich gewesen. Ich danke Euch, für Eure Liebe, Euren Zuspruch und Eure Unterstützung! Liebe Christina, auch wenn wir uns manchmal streiten wie die Kesselflicker weiß ich, dass ich mich zu 100\% auf Dich verlassen kann. Es ist schön solche Menschen um sich zu wissen. Du wirst immer meine kleine-große Schwester bleiben! Ich hab Dich ganz doll lieb und bin froh, dass es Dich gibt! Lieber Arif, danke für Deine Hilfsbereitschaft in allen Lebenslagen.

Liebe Marlies, lieber Laurenz, liebe Sandra, vielen Dank für Euer Interesse in meine Arbeit. Mit Eurer herzlichen Art habt ihr mich immer willkommen geheißen im Hasenwald und ich fühle mich sehr, sehr wohl bei Euch! 
Lieber Swen, dir sollen die letzten Worte meines Dankwortes zuteil werden. Ich möchte Dir für die letzten sechs Jahre danken in denen Du immer für mich da warst und mich unterstützt hast. Nie zuvor habe ich einen Menschen kennengelernt, der so bescheiden, ehrlich, hilfsbereit und liebenswürdig ist wie Du. Kurzum du hast das Herz am rechten Fleck und dafür liebe ich Dich. Ich bin froh Dich an meiner Seite habe. In Liebe, Silke! 


\section{About the author}

Silke Friederike Metzelthin was born on 5th May 1983 in Würselen (Germany). She attended the secondary school (Gymnasium) in Herzogenrath (Germany). After her graduation she studied Occupational Therapy at Zuyd University of Applied Sciences in Heerlen (the Netherlands). Her bachelor thesis was about the development of a self-management program for community-dwelling older people. After obtaining he bachelor's degree in June 2009, she followed the Health Sciences Research Master at Maastricht University (the Netherlands). At the same time she worked as student assistant at the Department of Health Services Research at Maastricht University. Her master thesis was about the validation of three frailty instruments. During the master she also developed a research proposal for a randomised controlled tria studying the feasibility, effectiveness and cost-effectiveness of an interdisciplinary primary care approach for community-dwelling frail older people. The proposal was approved by the Dutch Organisation for Health Research and Development (ZonMw) (grant number \#60-61900-98-213). Consequently, in August 2009 she has started as a PhD student on this research project under supervision of prof. dr. Ruud Kempen prof. dr. Luc de Witte and dr. Erik van Rossum.

In December 2012, Silke has received a travel grant awarded by Maastrich University, CAPHRI School for Public Health and Primary Care. Consequently, she visited Johns Hopkins University in Baltimore for nine weeks to work together with dr. Sarah Szanton and prof. dr. Laura Gitlin.

In April 2013, Silke has applied successfully for the 12 months Kootstra Talent Fellowship for future postdocs, awarded by Maastricht University. Her aim was to develop a community-based care approach for frail older people based on a philosophy of Function-Focused Care, a care philosophy developed by prof. dr. Barbara Resnick and colleagues from the University of Maryland. Within this philosophy caregivers learn to actively help older adults to achieve and maintain thei highest level of function based on the theory of self-efficacy, while taking into account environmental and policy issues. At the same time Silke worked successfully with Dutch colleagues from Maastricht University, UMC St Radboud, and UMC Utrecht on a proposal for a research line to improve basic nursing care. Part of this ZonMw funded research line (grant number \#80-80705-98-025) is an exploratory tria investigating the feasibility and effectiveness of a Function-Focused Care approach Consequently, Silke has started in January 2014 as a postdoctoral researcher at the Department of Health Services Research at Maastricht University. During the upcoming four years she will focus on the development and evaluation of a community-based care approach for frail older people, which is based on the philosophy of FunctionFocused Care. 


\section{List of publications}

Wi-1: scientific publications in international journals mentioned in the Social Science Citation Index, Science Citation Index of Arts and Humanities Citation with Impact Factor (IF)

Metzelthin, SF, Rossum, E van, Hendriks, MRC, Witte, LP de, Sipers, W, Hobma, S. Cost-effectiveness of an interdisciplinary primary care approach to reduce disability in community-dwelling frail older people: a cluster randomized controlled trial (under review).

Metzelthin SF, van Rossum E, de Witte LP, Ambergen T, Sipers W, Hobma S. Effectiveness of an interdisciplinary primary care approach to reduce disability in community-dwelling frail older people: a cluster randomised controlled trial. BMJ. 2013;347:f5264. IF=14.1 (1st decile)

Metzelthin SF, Daniëls R, van Rossum E, Cox K, Habets H, de Witte LP, Kempen GIJM. A nurse-led interdisciplinary care approach to prevent disability among community-dwelling frail older people: a large-scale process evaluation. Int J Nurs Stud. 2013;50(9):1184-96. IF=2.2 (1'st decile)

Daniëls R, Rossum HIJ, Metzelthin S, Sipers W, Habets H, Hobma S, van den Heuvel WIJ, de Witte LP. Rehabilitation in Practice: A disability prevention programme for community-dwelling frail older persons. Clin Rehabil.2011;25:1-12. IF=2.1 (1tt quartile)

Metzelthin SF, Rossum HIJ, de Witte LP, Marike RC Hendriks, Kempen GIJM. The reduction of disability in community-dwelling frail older people: design of a two-arm cluster randomized controlled trial. BMC Public Health. 2010;10:511. IF=2.2 (2 $2^{\text {nd }}$ quartile)

Metzelthin SF, Daniëls R Rossum HIJ de Witte $L P$ van den Heuvel WIJ, Kempen GIJM. The psychometric properties of three self-report screeningsinstruments for identifying frail older people in the community. BMC Public Health. 2010;10:176. IF $=2.2$ (2nd quartile)

Daniëls R, Metzelthin S, Rossum HUJ de Witte LP, Kempen GUMM van den Heuvel WIJ Interventions to 作 pren $=13$ (2nd quartile)

Wi-2: scientific publications in international journals with referee system and without Impact Factor including reviewed and internationally published abstracts

Metzelthin S. Rossum E van, Witte LP de, Kempen GIJM. Effects and feasibility of a Dutch interdisciplinary primary care approach for frail older people (abstract). Gerontologist. 2013; 53 (Suppl 1):415

Metzelthin S, Daniëls R, Rossum E van, Beurskens A, Heuvel WJA van den, Witte L de, Kempen GIJM. The psychometric properties of three self-report screening instruments for identifying community-dwelling frail elderly (abstract). Gerontologist. 2012;52 (Suppl 1): 280-281.

Metzelthin S, Rossum Evan, Daniëls R, Cox K, Habets H. Witte L de, Kempen GIJM. Disability prevention in frail older people: an interdisciplinary primary care approach (abstract). Gerontologist. 2012;52 (Suppl 1):305-306.

Metzelthin S, Daniëls R, Rossum E van, Witte LP de, Kempen GIJM. Disability prevention in frail older people: feasibility of an innovative interdisciplinary primary care approach (abstract). Late Breaker Poster Session Program Book GSA Meeting 2011, pp 29-30.

R. Witte L de, Heuvel W van den, Kenpen GIJM (2010). The psychometric properties of three self-report screening instruments for identifying frail older people in the community (abstract). Gerontologist. 2010;50 (Suppl 1):47. 
WN: scientific publications in national journals with referee system including published Dutch abstracts

Daniëls R, Rossum E, Metzelthin S, de Witte L. Zorg uit Voorzorg - Een preventief programma voor thuiswonende kwetsbare ouderen. Tijdschr Ouderengeneeskunde. 2012;6:298-303.

Metzelthin SF, Rossum HIJ, de Witte LP, Kempen GIJM. Het opsporen van thuiswonende kwetsbare ouderen met behulp van een tweetraps screeningsmethodiek (abstract). Tijdschr Gerontol Geriarts. 2012;43(4):212.

Metzelthin SF, Daniëls R, Rossum E van, Habets H, Witte LP de, Kempen GIJM. Reductie van beperkingen in activiteiten bij thuiswonende kwetsbare ouderen. Procesevaluatie van een innovatieve zorgannak in de eerste lijn: 'Zorg uit Voorzorg' (abstract). In: Programma en samenvattingen Geriatriedagen 2012:83-84.

Metzelthin S, Daniëls R, Rossum van E, Witte de LP, Kempen GIJM. Disability prevention in frail older people: a new interdisciplinary primary care approach (abstract). Tildschr Verpleegkunde. 2012;1:36.

Metzelthin S, Daniëls R, Rossum E van, Witte LP de, Kempen GIJM. Disability prevention in frail older people: a new interdisciplinary care approach (abstract). Abstract book of The 11th European Doctoral
Conference in Nursing Science 2011:67. etzelthin si

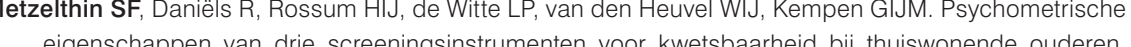
Tijdschr Gerontol Geriatr. 2011;42(3):120-130.

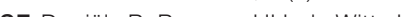

Metzelthin SF, Dane eigenschappen van drie screeningsinstrumenten voor kwetsbaarheid bij thuiswonende ouderen
(abstract). Tijdschr Gerontol Geriatr. 2010;41(4):39-40 (middenkatern). Daniëls R, Metzelthin S, Rossum HIJ, de Witte LP, Kempen GIJM, van den Heuvel WIJ. Interventies ter voorkoming van beperkingen bij thuiswonende kwetsbare ouderen: een systematische literatuurstuclume

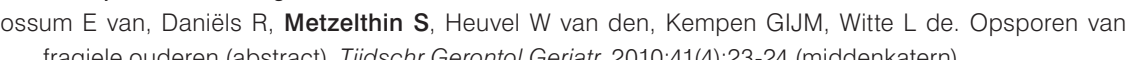
fragiele ouderen (abstract). Tijdschr Gerontol Geriatr. 2010;41(4):23-24 (middenkatern). 


\section{Appendix}

A disability prevention programme for community-dwelling frail older people

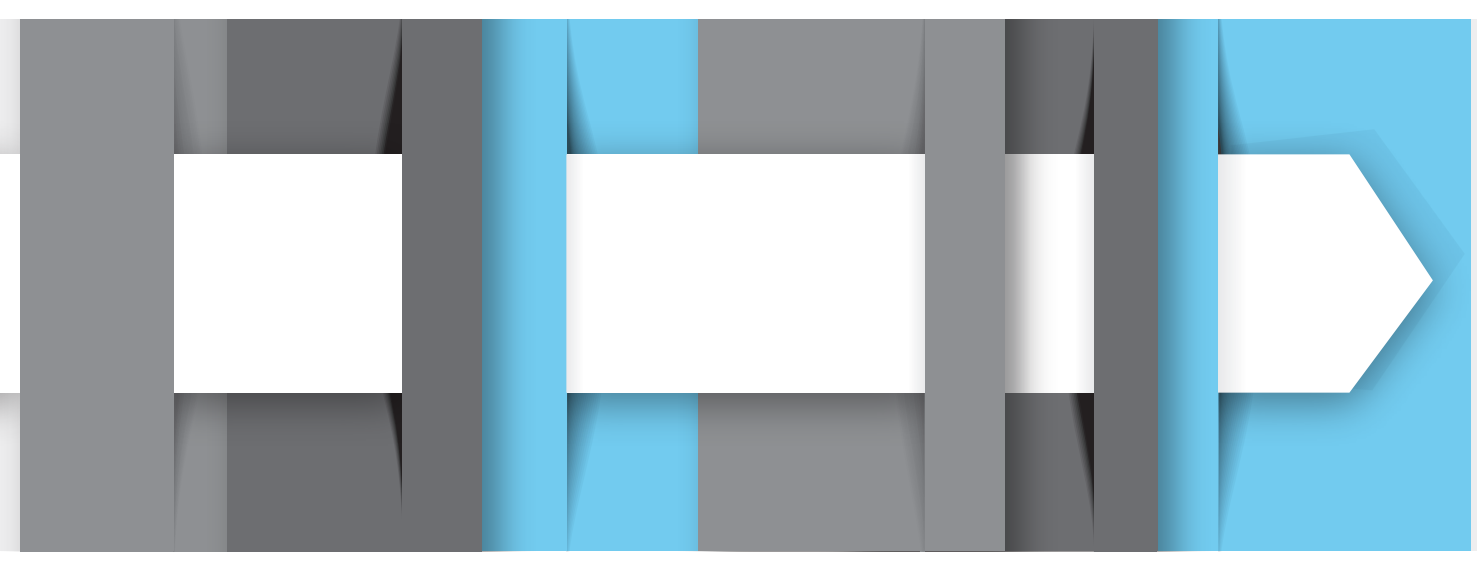

This chapter was published as: Daniëls, R, van Rossum, E, Metzelthin, S, Sipers, W, Habets, H, Hobma, S,

van den Heuvel, W, de Witte, L

Clinical Rehabilitation;25(11):963-974 


\section{Abstract}

Objective: To describe and justify a interdisciplinary primary care programme for community-dwelling frail older people aimed to prevent disability.

Background: Disability is a negative outcome of frailty among older people. Policy reports and research studies emphasise the need for programmes to reduce disability progression. Between 2008 and 2010 we developed such a programme.

Development: Following the Intervention Mapping protocol, a research team and a multidisciplinary task group developed the programme. Literature reviews and an expert meeting led to identification of basic elements, theory based methods and practical tools.

The programme: The general practitioner and the practice nurse comprise the core team that can be extended by other professionals such as the occupational and physiotherapist. The programme includes six steps: (1) screening, (2) assessment, (3) analysis and preliminary action plan, (4) agreement on action plan, (5) execution of the action plan (toolbox parts), and (6) evaluation and follow-up. The main features are: identifying risks for developing disability and targeting risk factors using professional standards and the $5 \mathrm{~A}$ Behavioural Change Model to support selfmanagement, and identifying problems in performing activities and enhancing meaningful activities based on the Model of Human Occupation. Screening and individual assessment, tailor-made and client-centred care, self-management support, case management and interdisciplinary cooperation are important principles in delivering the programme.

Discussion: The disability prevention programme seems promising for addressing the needs of frail older people for independent living and for targeting risk factors. Its feasibility and effects are being tested in an on-going randomised controlled trial.

\section{Introduction}

The rising number of frail older people poses various challenges for the public healthcare system. ${ }^{1}$ Frail older people are recognised as being at risk of adverse outcomes such as death, falls, hospitalisation and institutionalisation. ${ }^{2}$ Disability, defined as experiencing difficulty in performing activities in any domain of life ${ }^{3}$ is another negative outcome of frailty. Being independent and participating in society have great value for older people ${ }^{4}$

Supporting older people to live independently and participate in society are key policy targets for the Dutch government. ${ }^{5}$ Disability prevention for older people, in contrast to disease prevention, has recently been addressed by the Dutch Health Council $^{6}$ as function-oriented prevention. The Council emphasises the necessity for development and evaluation of tailor-made interventions that focus on promoting independent functioning in daily life for (vulnerable) older people with an important role for primary care, screening of vulnerable groups, and multidisciplinary cooperation.

Beswick and colleagues found that complex interventions like communitybased care can support older people to live independently, though there is no clea evidence yet that one specific format is better than another. Various recent studies on proactive home visits for vulnerable groups of older people in the Netherlands, for example, showed conflicting results. These home visits by nurses did not turn out to be effective ${ }^{8,9}$ or only modest positive effects were reported in the short term. ${ }^{10}$

A more powerful intervention in primary care is needed to address the needs of frail older people and prevent or postpone further functional decline. Although the Dutch healthcare system is characterised by its strong emphasis on primary care, ${ }^{11}$ it still seems insufficiently equipped to address the needs of frail older people and to prevent disability. ${ }^{6}$ The overall reactive approach and lack of collaboration between disciplines ${ }^{12,6}$ poses challenges in implementing complex interventions.

Between 2008 and 2010, we developed an interdisciplinary primary care disability prevention programme for frail older people. Between May 2009 and January 2010, feasibility study was conducted. ${ }^{13}$ Further, we studies the validity of the posta screening instrument used in the programme to identify frail older people. ${ }^{14,15} \mathrm{In}$ March 2010, sponsored by the Dutch National Care for the Elderly Programme, a full evaluation of the effectiveness of the programme started. ${ }^{16}$ This article aims to describe and justify the programme based on our development work.

Justification of the program - the theory

The development of the programme was based on the Intervention Mapping protocol $\left.\right|^{17}$ for developing health promotion programmes. Two teams cooperated during the development: a research team conducted (literature) studies and pilots to support decision making, and a multidisciplinary task group developed practica 
tools. The latter group consisted of professional experts such as a general practitioner, a nursing home physician, a geriatrician, a practice nurse, a geriatric clinical nurse specialist, a physiotherapist, an occupational therapist, an expert in technology and a researcher $(\mathrm{RD})$ as the coordinator.

\section{Defining frailty}

Although there is still debate on the definition of frailty, agreement exists on the core feature of frailty: an increased vulnerability to stressors due to impairments in multiple interrelated systems that lead to decline in homeostatic reserve and resiliency. ${ }^{18}$ Some authors describe frailty from as physiological perspective, ${ }^{19-24}$ also referred to as physical frailty, while others consider frailty as multifactorial in nature, taking physical, psychological, social and environmental factors into account..$^{25}$

Literature on disability development in older people suggests that disability is multifactorial in nature. Stuck et al. ${ }^{26}$ identified in their review the following risk factors for developing disability in the community-dwelling older people: cognitive impairment, depression, comorbidity, increased and decreased body mass index lower extremity functional limitation, falls, low frequency of social contact and low level of physical activity. Femia et al. ${ }^{27}$ suggest that although disease conditions and physical impairments are as risk factors strongly related to an individual's functiona abilities, other factors like the beliefs about one's health (e.g., subjective health) motivation and self-efficacy are potentially as important.

With disability prevention as the aim, a multifactorial approach to frailty was in ou opinion a more promising approach in identifying and supporting communitydwelling frail older people dealing with multiple diseases, problems and risks. As a consequence, the programme takes a multifactorial perspective on screening, assessment and treatment. As we consider frailty and disability as overlapping but distinct concepts, the programme needs to address the needs of frail older people in different stages from mildly frail (without disabilities) to severely frail.

Programme outcome and goals

The overall desired outcome of the programme is that frail older people can do those activities they need to do or enjoy doing. As the programme intends to be preventive in nature, two ways of supporting performance of activities were formulated:

- Identifying risks (as described by Stuck et al. ${ }^{26}$ ) for developing disability and targeting risk factors. Targets could be focused on cognitive impairment, depression, comorbidity, increased and decreased body mass index, lower extremity functional limitation, falls, low frequency of social contact or low level of physical activity.

- Identifying problems in performing activities and enhancing meaningful activities. The Model of Human Occupation ${ }^{28}$ describes determinants influencing performance of activities. The model was successfully used in previous effect studies.29,30 Goals could be focused on determinants such as personal causation (including self-efficacy), values, interests, roles, habits, performance capacity (mental and physical functions), skills (motor, cognitive, communicative) and the physical and social environment.

\section{Delivery of the programme}

Our literature review ${ }^{31}$ on interventions aimed at disability prevention in communiy-dwelling frail older people concluded that a tailor-made, multidisciplinary an multifactorial approach, individualised assessment and intervention, physical activity, assistive technology, case management and long-term follow-up are promising elements for a disability prevention programme. During an expert meeting (with sixteen researchers in geriatric care), experts recognised similar factors as self-management, a tailormade and client-centred approach, involvement of the client system and case management as relevant. Our programme elements are presented in Table 1.

Table 1 Elements of the disability-prevention programme

\begin{tabular}{|c|c|c|}
\hline General & Process & Intervention \\
\hline $\begin{array}{l}\text { - Disease and function- } \\
\text { oriented prevention } \\
\text { - Tailor-made } \\
\text { - Client-centred } \\
\text { - Supporting self-management } \\
\text { - Multifactorial approach to } \\
\text { frailty } \\
\text { - Multidisciplinary } \\
\text { cooperation }\end{array}$ & $\begin{array}{l}\text { - Screening } \\
\text { - Assessment } \\
\text { - } \text { Case management } \\
\text { Involvement of the client } \\
\text { system } \\
\text { - Multifactorial and flexible } \\
\text { toolbox of interventions } \\
\text { - Long-term follow-up }\end{array}$ & $\begin{array}{l}\text { Determinants: } \\
\text { Risk factor for developing } \\
\text { disabilities, e.g. } \\
\text { - Falls } \\
\text { - Mood problems } \\
\text { - Lack of physical activity } \\
\text { Determinants } \\
\text { Enhancing performance of } \\
\text { meaningful activities } \\
\text { - Performance } \\
\text { components (physical } \\
\text { - and mental functions) } \\
\text { - Personal causation } \\
\text { - Values } \\
\text { - Rnterests } \\
\text { - Roles } \\
\text { - Sabits } \\
\text { - Skills } \\
\text { - Pocial environment } \\
\text { (e.g., assistive } \\
\text { technology) }\end{array}$ \\
\hline
\end{tabular}


Tailor-made care and self-management support There is a strong body of evidence that self-management support, using a mixture of components is effective in improving clinical outcomes. ${ }^{32}$ The $5 \mathrm{~A}$ Behavioural Change Modele ${ }^{32,33}$ combines a client-centred approach, a model for behavioural change (Stages of Change model| ${ }^{34}$ ) and motivational interviewing techniques ${ }^{35}$ to provide practical tools for professionals to support self-management. Rubak and colleagues ${ }^{36}$ reported that motivationa interviewing outperforms traditional advice. The ' 5 As' refer to assessing the olde person's level of behaviour, beliefs and motivation for change; advice adapted to the need for information; agreeing with the older person on a realistic set of goals and actions (goal setting and action planning); assisting to anticipate barriers and to increase skills; and arranging follow-up support. ${ }^{32}$ Use of the $5 \mathrm{~A}$ Behavioural Mode implies that goals and strategies to achieve the goals are individually determined and will depend strongly on the older person's (self-perceived) problems, motivation and capabilities. Self-management skills of the older person will influence whether goals are focused on the client or more on (support of) the social and physical environment.

Meaningful activities As the programme aims to support older people in continuing to do those activities they enjoy or need to do, meaningful activities had to be at the core of the programme. The Occupational Performance Process Mode ${ }^{37}$ was helpfu in understanding that professionals need to explore concerns or problems of older people with performance of activities, understand the older person's priorities and use meaningful activities, where possible, as outcomes and means. The experience of doing can increase insight and beliefs in one's own capabilities (self-efficacy) ${ }^{28}$ which is central to self-management. Graff et al. ${ }^{30}$ showed the effectiveness of focus on meaningful activities in a programme for older people with dementia.

Interdisciplinary cooperation Besides having relevant clinical components, care programmes should also be seen as multidisciplinary protocols that encompas asks, decision criteria and work procedures for the care professionals involved Studies into team collaboration in rehabilitation ${ }^{39,40}$ show that a common care philosophy defined roles, open and clear communication, regular structural communication shared decision making and goal setting enhance team cooperation.

Delivering the programme - the practice

The GP and the practice nurse comprised the core team of the programme with the practice nurse in the role of case manager. This team can be extended to include the occupational therapist and physiotherapist, other community care professionals or hospital professionals. The programme consists of six steps (Figure $1^{16}$ ).
Screening: GP practice sends GFI to older people $(\geq 70)$.

GFI-score $\geq 5$ : PN calls for home visi

Assessment PN conducts assessment with older people (and informal caregivers) followed by decision making with GP about necessity for:

- Assessment OT and/ or PT (advised in case of concerns or problems in performing activities)

- Additional assessments

Analyses and action plan Perspective GP and PN

(or extended team including OT. PT and others) on curren

problems in performing activities and risk factors for

developing disabilities

Agreement on the action plan: home visit PN discusses

Agreement on the action plan: home visit PN discusses
with older people (and informal caregiver) goals, actions

and toolbox-parts

\section{Executing of the action plan (toolbox)}

- Meaningful activities (OT): increasing client's awareness of capacities, interests and self-efficacy in performing activities Adaptation of environment, skills or activities (PT and OT): adapting the environment (e.g. technology), learning new skills or new ways of performing activities. A tailor-made physical exercise program can be applied in case improvements in srength, balance, flexibility and endurance can contribute to social nerk tor disability

(Pocia Physical activity (PT): increasing physical activity in daily life Stimulate health (GP and PN): measures that will increase health and maintenance of a healthy lifestyle

Next to the toolbox, other interventions can be applied $\mathrm{PN}$ is case-manager monitoring progress and satisfaction

\section{Evaluation and Follow-up}

PN and older people (and caregiver) evaluate progress an agree on follow-up

Figure 1 Steps of the programme.

$\mathrm{PN}=$ practice nurse $\mathrm{OT}=\mathrm{occupational}$ therapist $\mathrm{PT}=$ physiotherapis
Multidisciplinary guidelines on geriatric problems - Dementia - Delirium - Polypharmacy - Fall prevention - Constipation and fecal incontinence - Urine incontinence - Depression

ion/ dehydration

Standards of

utch College of - Diabetes

Cardiovascular risk

management

Arthritis 
Step 1: Screening for frailty

The Groningen Frailty Indicator ${ }^{41}$ accompanied by a covering letter from the genera practice is sent to their population aged 70 or older. The Groningen Frailty Indicator ${ }^{4}$ is a 15-item screening instrument for determining the level of frailty. It focuses on the loss of functions and resources in four domains of functioning: physical (nine items), cognitive (one item), social (three items) and psychological (two items) Scores on the Groningen Frailty Indicator range from 0 to 15. The instrument has shown high internal consistency and construct validity ${ }^{15,41}$ In our feasibility study ${ }^{13}$ among 41 frail older people, nearly all older people were willing to return the Groningen Frailty Indicator by post ( $90 \%$ response), and the number of missing items was low. The study into predictive values of three postal questionnaires ${ }^{14}$ showed that all three instruments, including the Groningen Frailty Indicator, do have potential to identify older people at risk, but their predictive power is not yet sufficient. A substantia proportion of older people identified as frail by the Groningen Frailty Indicator did not develop adverse outcomes at one-year follow-up. In practice, this implies that additional information is needed to lower the number of false positives.

\section{Step 2: Assessment}

In the programme older people with scores $\geq 5$ on the Groningen Frailty Indicator are considered as frail and are contacted by phone by the practice nurse to plan a home visit. During this visit a multidimensional assessment is conducted by the practice nurse in the presence of the main informal caregiver (if available). This assessment ocuses on:

- concerns and wishes expressed by the older person and informal caregiver

- risk factors for disability (based on Stuck et al. ${ }^{26}$ ), such as polypharmacy, mobility problems, falls, lack of physical activity, cognitive impairments or mood problems

- problems or concerns experienced in performing activities

- readiness to change

A structured assessment format is used, based on various tools derived from instruments that have proven their use in previous research (e.g., the 'Easy-Care assessment' ${ }^{42}$ ). To optimise multidisciplinary communication, the format matches the structure of the electronic patient records of the geriatric department of the regional hospital.

Throughout the assessment the practice nurse uses motivational interviewing techniques ${ }^{35}$ to enhance collaborative partnership. Motivational interviewing focuses on providing opportunities to help patients assess for themselves what might be important or possible and how change might be achieved. ${ }^{35}$ Reflective listening accurately understanding a patient's story through open questions, reflections, gentle probing for more details, and use of summaries) is a key element for the practice nurse in conducting the multidimensional assessment. The Stages of Change mode ${ }^{34}$ is used in monitoring the readiness to change.

The stages of change are:

- Precontemplation: not yet acknowledging that there is a problem that needs to be changed

- Contemplation: acknowledging that there is a problem but not yet ready or sure of wanting to make a change

- Preparation: getting ready to change

- Action: changing behaviour

Maintenance: maintaining the behaviour change

- Relapse: returning to older behaviours and abandoning the new changes

\section{An example}

In answering the practice nurse's question about fall incidents, an older person tells that she fell three times, expresses her concern with these incidents, but does not seem to know what to change to improve the situation. The practice nurse concludes that, concerning fall incidents, the older person is in a contemplation phase ready to move to a preparation phase to receive information about possible actions to prevent falling.

In the end of the meeting, the practice nurse summarises the answers of the older person (and informal caregiver) and validates outcomes by asking:

- 'Could you tell me once more what are important activities for you to keep doing?'

- 'What would you say could support you in keep doing those activities as long as you wish?'

- How motivated are you to take measures that will help you to keep doing those activities?'

Practice nurses were told to use the assessment in a flexible manner and to focus on understanding the story of the older person and not on problem-solving. The pilot study ${ }^{13}$ showed that the assessment takes approximately one hour followed by hal an hour of administration. Older people were very positive about the opportunity to share their story and regarded the questions in the assessment as relevant. Although professionals considered the assessment to contain sufficient items about activities, doubt was expressed about whether older people were sufficiently challenged to reflect on their patterns of activities in the context of well-being and health. It is not usual for older people to express their problems and concerns in terms of activities, 
and more tools for professionals to discuss these kinds of issues with older people might be necessary.

Following assessment, the practice nurse and the GP discuss whether additiona assessments are needed. In the case of problems or concerns about performing activities, a referral to the occupational therapist and physiotherapist for an assessment is recommended to gain a better understanding of the underlying causes of problems experienced. Additional assessments by other specialized professionals follow if needed (and if agreed on by the older person).

Step 3: Analysis and preliminary action plan

As complex care needs may arise, thorough analyses of data available from assessments is necessary before action takes place. The GP and practice nurse discuss whether they can do the analysis and formulate a preliminary action plan together or whether the team has to be extended to include the occupational therapist and/ or physiotherapist and, other disciplines. In the case of an extended team, the professionals will held a team meeting using a format based on the Model of Human Occupation. ${ }^{28}$ The older person and the informal caregiver can be invited to the team meeting

The meeting takes a top-down approach towards analyses. After summarising the older person's background and context, the team identifies the activities meaningful to this person, the problems or concerns the older person expressed in performing activities, and the risk factors for developing disabilities. This is followed by analyses in which concepts as personal causation (including self-efficacy), values, interests, roles, habits, performance capacity (mental and physical functions), skills (motor, cognitive communicative) and the physical and social environment are taken into account.

After analyses, the team focuses on two questions:

- How can we support the older person to keep doing those activities that are meaningful?

- How can we support the older person to reduce the risk of developing disability in the near future?

The protocol offers specific information on a number of geriatric problems (e.g dementia, falls, depression, incontinence) based on recent standards and guidelines that professionals can use in their deliberations (see also figure $1^{16}$ ). The best course of action is discussed in perspective of the older person's needs, readiness for change and burden

\section{An example}

A team meeting could result in several goals and actions:

- Lowering the risk of developing disability using measures such as:

o polypharmacy: takes measures to increase safe use of medication toolbox 'stimulate health'.

o physical activity: increases physical activity in daily life toolbox 'physical activity'

o falls: takes measures to reduce fall risks toolbox 'adapting the environment, skills or activities'.

- Enhancing performance of (meaningful) activities such as:

o older person wishes to continue working in the garden: physical environment uses aids and adaptations to facilitate performance of activities toolbox 'adapting the environment, skills or activities'

o older person wishes to do meaningful activities in the weekend: interests: is able to explore interests and to choose for and perform meaningful activities toolbox 'meaningful activities'

During development of the programme, some professionals, especially GPs, were reluctant towards these team meetings. The pilot study showed some constraints in organising the meetings in one GP. The twelve practices in the randomised controlled trial, however, did not report problems with organisation so far. Interdisciplinary meetings usually took one hour in which three or four older people were discussed. Professionals experienced several advantages of this team collaboration, such as a more extensive picture of older people, sharing a mutual view on treatment, and better understanding of the expertise of other disciplines. The feasibility study showed that education and guidance of teams may be important factors in achieving effective eam collaboration.

Step 4: Agreement on action plan

The next phase relates to goal setting and action planning together with the older person (and informal caregiver). During a second home visit, the practice nurse explains that she would like to talk about measures to support the older person to keep doing meaningful activities. The practice nurse uses the motivational interviewing tool 'agenda setting' to raise issues considered important by the older person, the informal caregiver and the team. In agenda setting, rather than impose the professionals' priority on patients, one conducts an overview by inviting patients to select an issue or behaviour that they are most ready and able to tackle, feeling free also to express one's own views. ${ }^{35}$ 


\section{An example}

Using the tool 'Agenda setting' the practice nurse asks: 'Is there some other topic that you would prefer to talk about? l'd like to talk at some point about your fear of falling and your wish to keep doing gardening. But what makes sense to you right now?

After an agenda has been set, the older person's topics are discussed, followed by the topics of the practice nurse. Information and advice adapted to the needs of the older person (e.g. about possible treatment options) is given after permission has been asked. Further, the practice nurse can use motivational interviewing techniques such as 'reflecting on pros and cons of change' and 'assessing importance and confidence in change'

The outcome of this meeting is a list of goals, actions and responsibilities that should meet the older person's and informal caregiver's needs. It is not important for practice nurses to make time for this meeting, and do this as a face-to-face interaction and not over the phone. The practice nurse needs to involve the older person in decision making, and goals of treatment should be specific so that they motivate and direct a person's attention toward goal relevant activities. It is not important that all issues seen as important by the team are negotiated. The setting of goals and actions is a part of a process in which professionals and clients establish a cooperation, in which a learning process begins, leading to new insights and possibly to new goals and actions. Practice nurses in the pilot study experienced that goal setting in this programme differs from goal setting with clients with chronic obstructive pulmonary disease (COPD) or diabetes. They reported that client goals in our programme range further in scope and are less pre-described.

Step 5: Executing the action plan

A flexible toolbox of interventions is available to execute the action plan. For each toolbox part, a rationale and method are described to guide professionals. The toolbox exists of five parts:

Meaningful activities (occupational therapist). This part of the toolbox is meant for older people who lack confidence in capabilities or experience a lack in activities tha they enjoy to do or that give meaning to life. The objective is to explore capacities, interests and satisfaction with meaningful activities and ability to choose and perform meaningful activities. The method is based on occupational therapy literature ${ }^{28}$ motivational interviewing ${ }^{35}$ and the Stages of Change model. ${ }^{34}$
Adapting the environment, activities or skills (occupational and physiotherapist). This module is for older people who experience problems in performance of activities. The objective is to adapt the environment, activities or skills to enhance performance of activities. The method is based on standards and guidelines in occupational and physiotherapy for advising assistive technology and strategy training. A functiona exercise program (based on Gill et al. ${ }^{43}$ ) focusing on physical parameters as strength balance, endurance and flexibility is also part of this module.

Social network and social activities (practice nurse). This part of the toolbox is for older people with a small or instable network, without sufficient support from their network, experiencing tension in their social network or loneliness. The objective is to strengthen the social network and/ or increasing social activities. The method is based on the Dutch 'strengthening your network' programme, as described by Hofman et al. ${ }^{44}$ and the 'friendship course' by Stevens et al ${ }^{45}$. Increased insight in one's network and wishes in relation to the social network are translated into goals and an action plan.

Daily physical activity (physiotherapist). This part is meant for older people aiming to increase daily physical activity. The method is based on motivational interviewing the Stages of Change model and the seven-step approach as described by Resnick et al.

Stimulate health (GP and practice nurse). This module is for older people with chronic diseases or other risk factors for developing disability related to lifestyle. The objective is to take measures that stimulate health and a healthy lifestyle based on standards of the Dutch College of General Practitioners. In case of behavioural change, the GP and practice nurse use motivational interviewing and the Stages of Change model to guide the older person.

During execution of the toolbox parts the case manager (practice nurse) keeps in touch with the older person and the informal caregiver to monitor progress and satisfaction.

The pilot study ${ }^{13}$ showed that professionals considered this toolbox appropriate or targeting frail older people. The methods in the toolbox required the professionals to adapt. They had to change their focus from problem-solving to support the client's self-management and increase their own coaching skills.

Step 6: Evaluation and follow-up

After finishing parts of the toolbox, the practice nurse evaluates, as case manager with the older person the achievement of goals, the implementation of strategies in daily life and the need for support in the following period. This support could be arranged, for example, through regular visits, telephone, email or community-based 
facilities. The professionals involved will be informed about the agreements. It is possible, for example, that an older person who was involved in the toolbox part 'physical activity' still has a monthly telephone contact with the physiotherapist to support the maintenance of new habits.

From the perspective of behavioural change, follow-up is a relevant phase. As a result of events and transitions, frail older people can have difficulty in maintaining self-management strategies. In the pilot study ${ }^{13}$, arranging the follow-up was one of the elements that was often not applied. Arranging a follow-up is a rather new element in primary care; it is not in the process of usual care and usually there is no reimbursement for it. A good system is needed to organise who is responsible, for what, in which period. This needs more attention in the programme.

\section{Discussion}

This paper describes the content of a disability-prevention programme for community dwelling frail older people. The programme was designed to promote self-management in a tailor-made way using motivational interviewing and meaningful activities as a vehicle to guide behavioural change. Literature studies provided relevant elements in delivering the programme. To shape the programme, the research team and the multidisciplinary task group used theories, models and tools that have proven their usefulness in other contexts. How this combination works out in the context of community-based care targeted at frail older people is still unclear. Bodenheimer ${ }^{32}$

for instance, emphasises that although there is a strong body of evidence that self-management support is effective in achieving clinical outcomes, much work is still needed on precisely which activities are the most effective for which patients. Jonker and colleagues ${ }^{47}$ found in their review that a group based Chronic Disease Self-Management Programme for vulnerable older people (containing goal setting and action planning) was consistently beneficial for health behaviour, especially with regard to the variables of exercise and self-care. How effective self-management support is in an individual format in community-dwelling frail older people is a question for further studies.

The combination of elements, theories, models and tools has led to a complex intervention. New elements such as screening, motivational interviewing, meaningfu activities in the core, team meetings and a toolbox of interventions might be too ambitious for the average GP practice. The pilot study ${ }^{13}$ showed that the screening procedures needs reconsideration and that additional tools for professionals to support self-management are necessary.
Acknowledgements

For their contribution to developing the disability prevention programme many thanks go to Inge Systermans, Margot van Melick, Frederique Prompers, Frank Vlaskamp Henk Jochems, Rob de Ruijter and Michel Oostdam. 


\section{References}

1. Hirdes JP. Addressing the health needs of frail elderly people: Ontario's experience with an integrated health information system. Age Ageing. 2006:35(4):329-31.

Ferrucci L, Guralnik JM, Studenski S, Fried LP, Cutler GB, Jr., Walston JD. Designing randomized, controlled trials aimed at preventing or delaying functional decline and disability in frail older persons: consensus report. J Am Geriatr Soc. 2004;52(4):625-34.

3. Jette AM. Toward a common language for function, disability and health. Phys Ther, 2006:86(5):726-34 Swedish Nationa Institute of Public Health. Heathy ageing -achal National Institute of Public Health 2007.

5. Ministerie van Volksgezondheid WeS. Nota 'Ouderenbeleid in het perspectief van de vergrijzing' Den Haag: Ministerie VWS; 2005

RVZ Preventie bï ouderen: focus op zelfredzaamheid. Den Haag: Gezondheidsraad2009

7. Beswick AD, Rees K, Dieppe P, Ayis S, Gooberman-Hill R, Horwood J, Ebrahim S. Complex interventions to improve physical function and maintain independent living in elderly people: a systematic review and eta-analysis. Lancet. 2008:371(9614):725-35.

8. Van Hout HP. Jansen AP, van Marwijk HW. Pronk M, Frijters DF, Nijpels G. Prevention of adverse health trajectories in a vulnerable elderly population through nurse home visits: a randomized controlled trial. Gerontol A Biol Sci Med Sci. 2010;65(7):734-42.

9. Nicolaides-Bouman A, van Rossum E, Kempen GI, Knipschild P. Effects of home visits by home nurses to elderly people with health problems: design of a randomised clinical trial in the Netherlands (1) 2004:4(1):35.

10. Melis RJ, van Eijken MI, Teerenstra S, van Achterberg T, Parker SG, Borm GF, van de Lisdonk EH, Wensing M, Olde Rikkert MG. A randomized study of a multidisciplinary program to intervene on geriatr syndromes in vulnerable older people who live at home Kea SCi. $2008,63(3) .283-90$.

11. Knottnerus JA, ten Velden GH. Dutch doctors and their patients - effects of health care reform in the Netherlands. N Eng/ J Med. 2007;357:2424-6.

2. Van Oosterbos $\mathrm{H}$. The future of integrated primary care: community health centres at the heart of the neighbourhood. Int J Integr Care. 2006;6:e08

3. Daniels R. Frail Elderly - Identification and disability prevention in primary care [PhD Thesis]. Maastrich School for Public Health and Primary Care, Maastricht University; 201

14. Daniels R, Van Rossum HIJ, Beurskens A, Van den Heuvel W, De Witte L. The predictive validity of three self-report screening instruments for identifying frail older people in the community. BMC Public Health. 2012;12:69.

15. Metzelthin SF, Daniels R, van Rossum E, de Witte L, van den Heuvel WJ, Kempen Gl. The psychometric properties of three self-report screening instruments for identifying frail older people living in the community BMC Public Health. 2010;10:176

16. Metzelthin $\mathrm{SF}$, van Rossum E, de Witte LP, Hendriks MR, Kempen GI. The reduction of disability in community-dwelling frail older people: design of a two-arm cluster randomized controlled trial. BMC Public Health. 2010;10(1):511.

17. Bartholomew LK, Parcel GS, Kok G, Gottlieb NH. Planning Health Promotion Programs: Intervention Mapping. San Francisco: Jossey-Bass; 2006.

18. Rockwood K. What would make a definition of frailty successfull? Age Ageing. 2005;34:432-4

19. Fried $L P$, Ferrucci $L$, Darer J, Williamson JD, Anderson G. Untangling the concepts of disability, frailty and comorbidity: implications for improved targeting and care. J Gerontol A Biol Sci Med Sci. 2004;59(3):255-63.

20. Fried LP, Tangen CM, Walston J, Newman AB, Hirsch C, Gottdiener J, Seeman T, Tracy R, Kop WJ, Burke G, McBurnie MA. Frailty in older adults: evidence for a phenotype. J Gerontol A Biol Sci Med So 2001;56(3):M146-56.

21. Rockwood K, Fox RA, Stolee P, Robertson D, Beattie BL. Frailty in elderly people: an evolving concept. CMAJ. 1994;150(4):489-95
2. Walston J. Fried LP. Frailty and the older man. Med Clin North Am. 1999:83(5):1173-94.

3. Buchner DM, Wagner EH. Preventing Frail Health. Clin Geriatr Med. 1992;8(1):1-17.

24. Fried LP. Frailty. In: Hazzard W.R. B, R.L. Blass, J.P., Ettinger, W.H., Halter, J.B., ed. Principles of geriatric medcine and gerontology. 4th edn ed. New York, NY, USA: McGraw Hill; 1994:1149-56.

25. Bergman H, Ferrucci L, Guralnik J, Hogan DB. Hummel S, Karunananthan S, Wolfson C. Frailty: an chal 2007;62(7):731-7.

26. Stuck AE, Walthert JM, Nikolaus T, Bula CJ, Hohmann C, Beck JC. Risk factors for functional status decline in community-living elderly people a systematic literature review Soc Sci Med 1999:48(4):445-69.

27. Femia $\mathrm{EE}$, Zarit $\mathrm{SH}$, Johansson $\mathrm{B}$. The disablement process in very late life: a study of the oldest-old in Sweden. J Gerontol B Psychol Sci Soc Sci. 2001;56(1):P12-23.

28. Kielhofner G. Model of Human Occupation. Theory and application. Baltimore: Kluwer/Lippincot Williams\&Wilkins Wolters; 2008.

29. Corr S, Bayer A. Occupational therapy for stroke patient after hospital discharge-a randomized controlled trial. Clin Rehabil. 1995;9:291-6.

30. Graff MJ, Vernooii-Dassen MJ, Thijssen M, Dekker J, Hoefnagels WH, Olde Rikkert MG. Effects of community occupational therapy on quality of life, mood, and health status in dementia patients and the caregivers: a randomized controlled trial. J Gerontol A Biol Sci Med Sci. 2007;62(9):1002-9.

31. Daniels R, Metzelthin $S$, Van Rossum E, De Witte L, Van den Heuvel W. Interventions to prevent disability in frail community-dwelling older persons: an overview Eur J Ageing 2010;7(1):37-55.

32. Bodenheimer T. Grumbach K. Improving primary care. Strategies and tools for better practice. New York: Lange Medical Books/McGraw-Hill; 2007.

33. Glasgow RE, Emont S, Miller DC. Assessing delivery of the five 'As' for patient-centered counseling Health Promot int. 2006;21(3):245-55.

Jente C. Stages and processes of self-change in smoking: toward an integrative model of change. Journal of Consulting and Clinical Psychology. 1983;5:390-5.

35. Miller WR, Rollnick S. Motivational interviewing: preparing people for change. New York: Guilford Publications; 2002

36. Rubak S, Sandbaek A, Lauritzen T, Christensen B. Motivational interviewing: a systematic review and meta-analysis. Br $J$ Gen Pract. 2005;55(513):305-12

37. Townsend E, Stanton S. Enabling occupation: an occupational therapy perspective. Ottawa2002.

8. Jansen $\mathrm{YJ}$, de Bont A, Foets $\mathrm{M}$, Brujijzzeels M, Bal R. Tailoring intervention procedures to routine primary health care practice; an ethnographic process evaluation. BMC Health Serv Res. 2007;7:125.

39. Gibbon B. An investigation of interprofessional collaboration in stroke rehabilitation team conferences. Clin Nurs. 1999;8(3):246-52.

40. Nijhuis BJ, Reinders-Messelink HA, de Blecourt AC, Olijve WG, Groothoff JW, Nakken H, Postema K. A review of salient elements defining team collaboration in paediatric rehabilitation. Clin Rehabil. 2007;21(3):195-211.

41. Steverink N, Slaets JPJ, Schuurmans H, van Lis M. Measuring frailty: development and testing of the Groningen Frailty Indicator (GFI). Gerontologist. 2001;41(1):236-7.

42. Richardson $\mathrm{J}$. The Easy-Care assessment system and its appropriateness for older people. Nurs Older People. 2001;13(7):17-9.

43. Gill TM, Baker DI, Gottschalk M, Peduzzi PN, Allore H, Byers A. A program to prevent functional decline in physically frail, elderly persons who live at home. N Eng/ J Med. 2002;347(14):1068-74.

44. Hofman E, Scherpenzeel R. Netwerk rond cliënt versterken. Bunnik: Mezzo; 2008.

45. Stevens N, Albrecht H. De vriendschapscursus voor oudere vrouwen. Nijmegen: Radboud University/ Centre of Psychogerontology; 1995

46. Resnick B. A seven step approach to starting an exercise program for older adults. Patient Educ Couns. 2000;39(2-3):243-52.

47. Jonker AA, Comijs HC, Knipscheer KC, Deeg DJ. Promotion of self-management in vulnerable older people: a narrative literature review of outcomes of the Chronic Disease Self-Management Program (CDSMP). Eur J Ageing. 2009;6(4):303-14. 